\title{
SIMULACIÓN DE FLUJOS COMPRESIBLES UTILIZANDO UN ESQUEMA EXPLÍCITO DE TAYLOR-GALERKIN MODIFICADO.
}

Tesis presentada por:

\section{Horacio Pedro Burbridge.}

ante la Facultad de Ingeniería de la Universidad Nacional de La Plata para acceder al grado académico de:

\section{DOCTOR EN INGENIERIA}

Director de tesis: Dr. Armando Miguel Awruch.

Codirectora: Dra. Ana Elena Scarabino.

Jurado:

Dr. Guillermo O. Artana

Dra. Victoria C. Vampa

Dr. Sergio A. Elaskar

La Plata, 16 de octubre de 2020 


\section{AGRADECIMIENTOS}

Deseo agradecer a las siguientes personas e instituciones por su ayuda y apoyo para la realización de esta tesis.

A mis directores de tesis, Armando Miguel Awruch y Ana Elena Scarabino por su apoyo constante para la realización de este trabajo. Especialmente a Ana por su paciencia y contribución en la dirección y selección de alternativas de desarrollo en los momentos más difíciles.

A la UNLP por admitirme para la realización de este trabajo.

Al CEDI de la UTN FRH por el financiamiento de gran parte de este trabajo y por el apoyo de los colaboradores Andrés Trapanotto y Juan Carlos Polidoro.

A la ex jefa del área de simulación y modelado de YPF Tecnología, Marcela Raviculé, por considerar este trabajo dentro del plan de formación de la empresa.

A mi madre por el apoyo y el amor brindados durante toda mi vida y por el soporte que permitió desarrollar mi formación.

Finalmente, a Camila y a Violeta, por el amor de todos los días, y por "aguantarme" con paciencia todo este tiempo mientras desarrollaba este trabajo. 


\section{RESUMEN}

Como ya fue demostrado por diferentes autores, el esquema de Taylor-Galerkin (TG) en el contexto del Método de Elementos Finitos (FEM: Finite Element Method) resulta particularmente adecuado para la solución de flujos compresibles en régimen supersónico que involucran ondas de choque. Sin embargo, el esquema TG presenta inestabilidades en flujos con números de Mach subsónicos. Aún en casos en los cuales el Mach freestream es supersónico, siempre existen regiones del flujo, cerca de las paredes de los obstáculos, en donde la velocidad es baja o próxima a cero y el Mach local es subsónico. Por esto, algunos investigadores desarrollaron el algoritmo "Characteristic Based Split" (CBS) buscando obtener un único esquema que presente un comportamiento adecuado, tanto en regímenes subsónicos como supersónicos. En las últimas dos décadas algunos trabajos establecieron ciertas ventajas en la convergencia del algoritmo CBS comparado con el algoritmo TG. Sin embargo, estas ventajas de convergencia son a costo de tiempo de simulación debido a la operación de "Split" típica del algoritmo CBS. En este trabajo se propone un esquema híbrido denominado Taylor-Galerkin Modificado (MTG: Modified Taylor-Galerkin) que presenta las ventajas de ambos esquemas: mejor convergencia sin costo adicional en términos de tiempo de cómputo. Se aplicaron los esquemas TG, CBS y MTG utilizando elementos finitos hexaédricos en conjunto con una técnica de captura de choque para la solución de flujos aerodinámicos compresibles supersónicos, viscosos y no viscosos. Además, con la intención de obtener un algoritmo eficiente, las matrices elementales son integradas analíticamente. Ésto se realiza con dos enfoques diferentes. En el primer enfoque, la matriz Jacobiana inversa y el determinante de la matriz Jacobiana a nivel de elemento se evalúan con una forma de integración reducida, utilizando el punto situado en el centro del elemento para las matrices de masa, convectivas, difusivas y matrices de estabilización; todas estas matrices están integradas analíticamente usando el Jacobiano en el centro del elemento. En el segundo enfoque, las matrices de masa y convectivas se calculan mediante un esquema de integración completa donde se integra considerando la dependencia del Jacobiano con la posición, mientras que sólo las matrices difusivas y las matrices de estabilización se calculan con integración reducida, utilizando el punto situado en el centro del elemento para calcular la matriz Jacobiana inversa y el determinante de la matriz Jacobiana a nivel de elemento. Por último, este trabajo incorpora el modelo de turbulencia de Spalart-Allmaras, pero utilizando una versión conservativa de la ecuación de transporte de la variable turbulenta. Se prueban los algoritmos para determinar las mejoras de convergencia tanto en casos laminares como turbulentos para diferentes números de Mach (flujos, subsónicos, transónicos y supersónicos). 


\section{ABSTRACT}

As already shown by different authors, the Taylor-Galerkin (TG) scheme, in the context of the Finite Element Method (FEM), is particularly suitable for the solution of supersonic flows. However, the TG scheme usually presents instabilities in subsonic flows. Even in cases in which the free stream Mach number corresponds to supersonic flows, there are always flow regions, specifically near the walls of the immersed obstacles, where the speed is lower, and the local Mach number corresponds to a subsonic flow. The Characteristic Based Split (CBS) scheme was developed to obtain a single method to improve the behavior with respect to TG method in subsonic and supersonic regimes. In the last two decades some works have shown advantages in convergence rates of the CBS method when compared to the TG algorithm. However, simulation time increases in the CBS method since split operations, typical of this algorithm, imply in additional element loops. A hybrid algorithm called Modified-TaylorGalerkin scheme (MTG) is proposed here. This algorithm presents advantages with respect to TG and CBS schemes in terms of convergence properties and computational processing time. The TG, CBS and MTG schemes, using hexahedral finite elements, are applied in this work. A shock capturing technique for the solution of supersonic viscous and non-viscous compressible flows are also employed. To get an efficient algorithm, the element matrices are analytically integrated. This is performed with two different approaches. In the first approach the inverse matrix and the determinant of the Jacobian matrix at element level are evaluated with a reduced integration form, using the point located in the center of the element for mass, convective, diffusive and stabilization element matrices; all these matrices are integrated analytically. In the second approach, mass and convective matrices are calculated by a complete integration scheme (including the inverse matrix and the determinant of the Jacobian matrix at element level in the analytical expression to be integrated) and the diffusive and stabilization matrices are calculated with reduced integration, using the point located at the center of the element to calculate the inverse matrix and the determinant of the Jacobian matrix at element level. Finally, this work incorporates the Spalart-Allmaras (SA) turbulence model using a conservative version of the transport equation, as proposed by the authors of the original SA model in a later paper. Algorithms are tested to determine convergence rate improvements in both laminar and turbulent cases and for different Mach numbers (supersonic, transonic and subsonic flows). 


\section{NOMENCLATURA}

\section{LISTA DE SÍMBOLOS PRINCIPALES}

\section{Letras romanas mayúsculas}

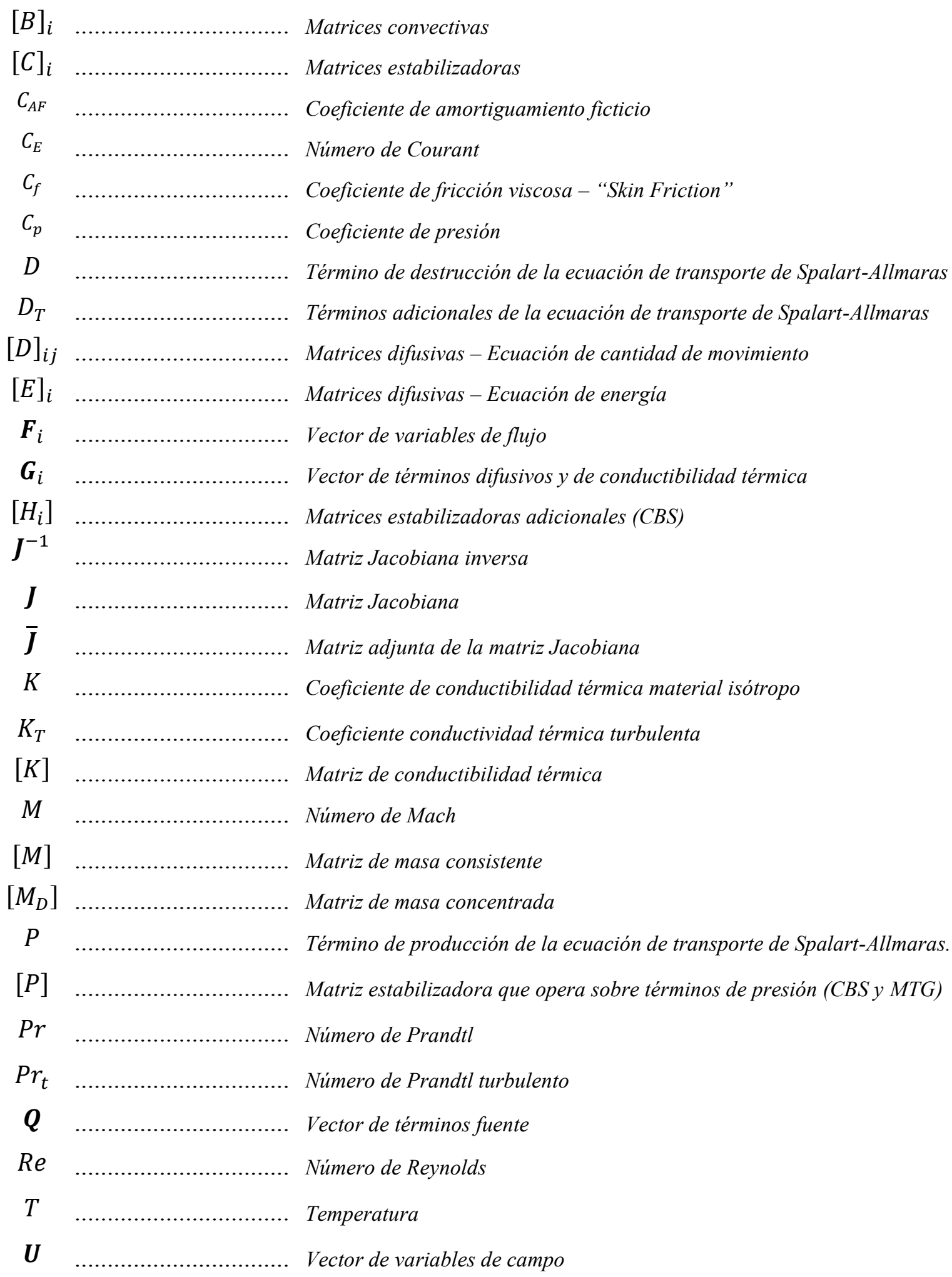




\section{Letras romanas minúsculas}

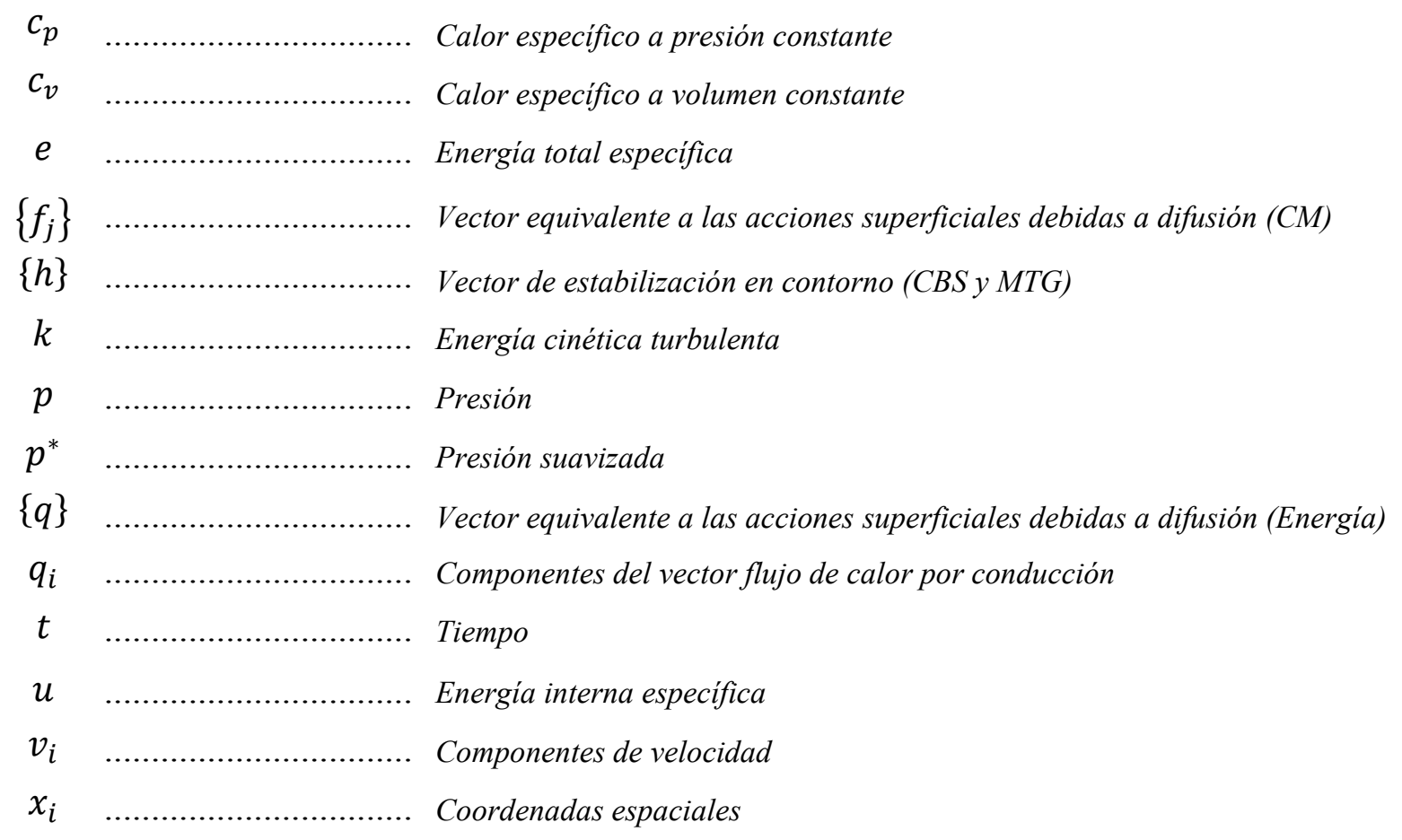

\section{Letras griegas mayúsculas}

$\Phi_{N}$

$[\Phi]$

$\left[\Phi^{*}\right]$

$\Gamma$

$\Omega$

\section{Letras griegas minúsculas}

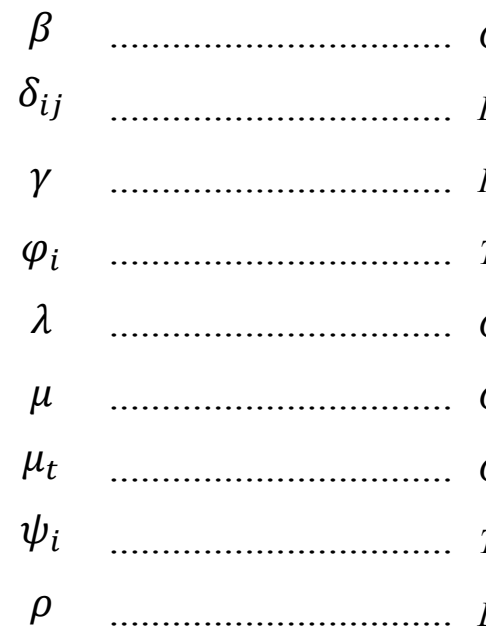

Funciones de interpolación

Matriz línea de funciones de interpolación

Matriz línea de funciones de interpolación evaluadas en el contorno

Dominio superficial - Contorno

Dominio volumétrico

Coeficiente de seguridad del incremento de tiempo crítico

Delta de Kronecker - Componentes del tensor de Kroncker

Relación entre los calores especificos - Constante del gas

Término difusivo de la ecuación de transporte de Spalart-Allmaras

Coeficiente de viscosidad volumétrica

Coeficiente de viscosidad

Coeficiente de viscosidad turbulenta

Término difusivo asociado a la energía cinética turbulenta (k) (ec. energía)

Densidad 


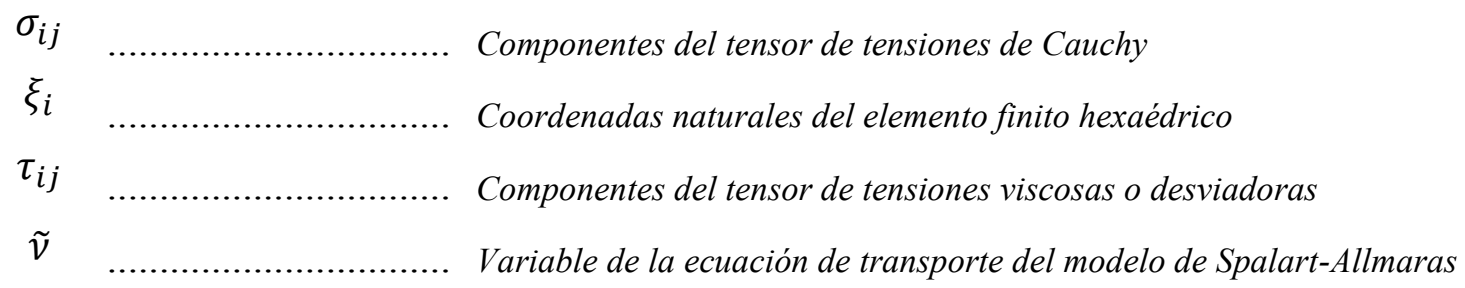

\section{CONVENCIONES}

Aquí se presentan las convenciones adoptadas en este trabajo y algunos ejemplos de su aplicación:

a) Los subíndices que constan de letras minúsculas indican las componentes espaciales de la variable o cantidad física a la que pertenecen $y$, a menos que se indique lo contrario, corren de 1 a 3, por ejemplo, $i, j, k=1.2,3$. Estos subíndices siguen la convención de suma de Einstein. Es decir, en cualquier expresión en la que aparezcan subíndices repetidos en el mismo término, debe considerarse que hay una suma desarrollada para este subíndice. La excepción de esta regla se indica colocando el subíndice entre paréntesis. Por lo tanto, cuando uno de los subíndices repetidos aparece entre paréntesis, significa que no se debe aplicar la convención de suma. Esta notación se ilustra a través de los siguientes ejemplos:

$$
\begin{gathered}
a_{i} b_{i}=a_{1} b_{1}+a_{2} b_{2}+a_{3} b_{3} \\
a_{i} b_{(i)} c_{j j}= \begin{cases}a_{1} b_{1}\left(c_{11}+c_{22}+c_{33}\right) & \text { para } i=1 \\
a_{2} b_{2}\left(c_{11}+c_{22}+c_{33}\right) & \text { para } i=2 \\
a_{3} b_{3}\left(c_{11}+c_{22}+c_{33}\right) & \text { para } i=3\end{cases}
\end{gathered}
$$$$
(i, j=1,2,3)
$$

b) Los subíndices formados por las letras mayúsculas $M$ y $N$ indican los valores nodales de la cantidad o variable física a la que pertenecen. A menos que se indique lo contrario, se aplican a los elementos de los vectores de elemento y se ejecutan de 1 a 8 . Estos subíndices no siguen la convención de suma de Einstein y, cuando hay una suma desarrollada para este subíndice, esto se indica utilizando el símbolo de suma correspondiente.

c) El subíndice $E$ indica que la cantidad que toma el subíndice se refiere al elemento finito. Este subíndice se utiliza cuando las cantidades físicas del elemento deben distinguirse de las cantidades físicas globales correspondientes, pero se omite cuando esa distinción no es indispensable para la comprensión del texto. 
d) El superíndice $\mathrm{n}$ indica el paso de tiempo cuando hace referencia a la variable a la que pertenece. Por lo tanto, por ejemplo, $a^{n}$ es la variable $a$ en el paso de tiempo "anterior", mientras que $a^{n+1}$ es la variable $a$ en el paso de tiempo "actual". El incremento correspondiente al tiempo actual se indica a través de la expresión $\Delta a^{n+1}=a^{n+1}-a^{n}$. 
ÍNDICE:

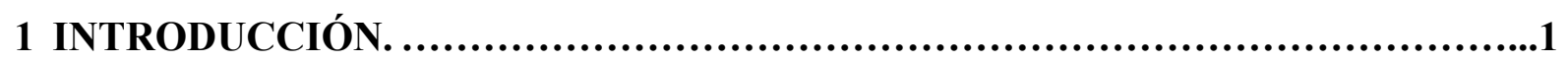

1.1 LA DINÁMICA DE FLUIDOS COMPUTACIONAL ................................................ 1

1.2 DIFERENTES MÉTODOS NUMERICOS. ............................................................. 5

1.3 LÍNEA DE INVESTIGACIÓN DE ESTA TESIS ....................................................... 7

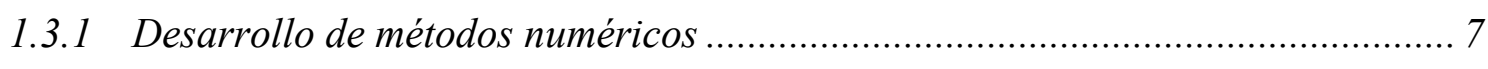

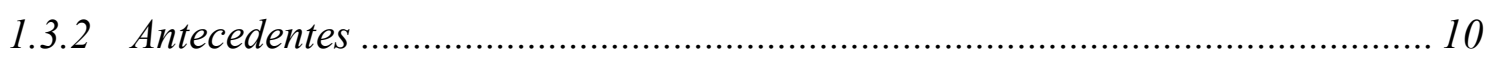

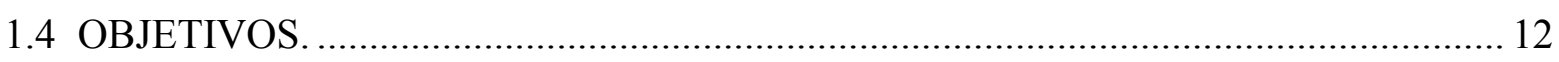

1.5 RECUSOS DISPONIBLES Y MEDIOS DE FINANCIAMIENTO_............................ 14

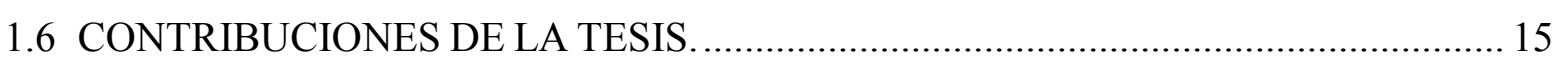

1.6.1 Contribuciones al avance científico y tecnológico. ........................................ 15

1.6.2 Contribuciones a la formación de recursos humanos. ..................................... 16

2 ECUACIONES DE GOBIERNO CON UN MODELO DE TURBULENCIA. ........18

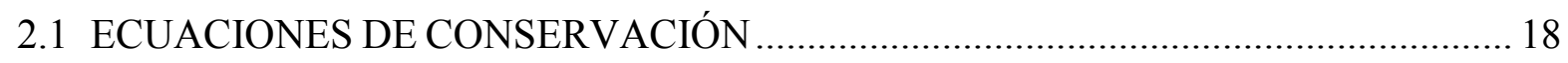

2.2 RELACIONES CONSTITUTIVAS: FLUIDO NEWTONIANO................................. 19

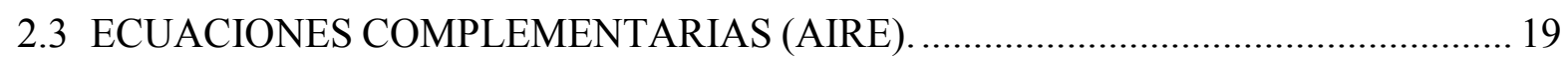

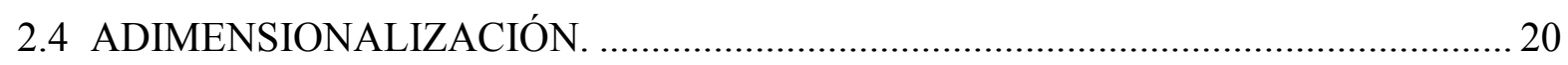

2.5 ECUACIONES FANS (FAVRE AVERAGED NAVIER-STOKES) .............................. 21

2.5.1 Promediado de las ecuaciones de Navier-Stokes: ............................................. 21

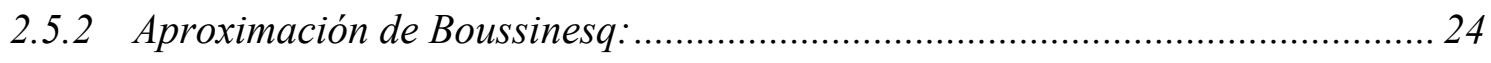

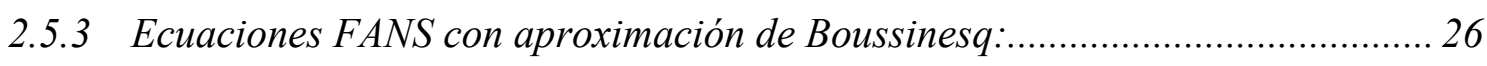

2.6 MODELO DE TURBULENCIA DE SPALART-ALLMARAS .................................... 27

2.7 ECUACIONES FANS INCLUYENDO MODELO DE SPALART-ALLMARAS EN

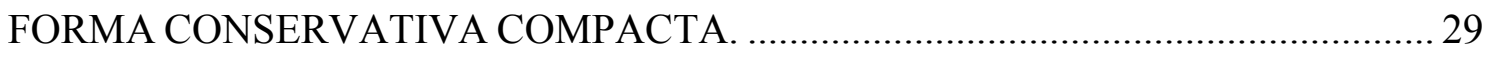

3 FORMULACIONES DE ELEMENTOS FINITOS. .............................32

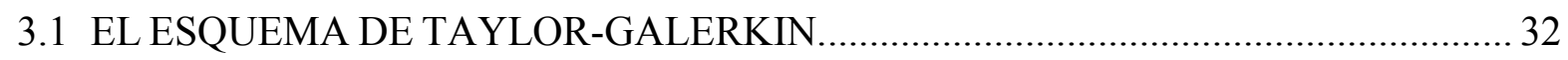

3.1.1 Avance en el tiempo: serie de Taylor............................................................ 32

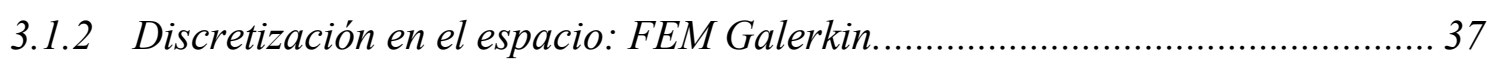

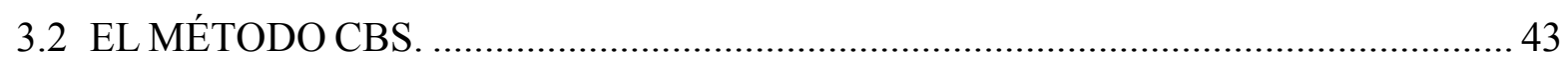

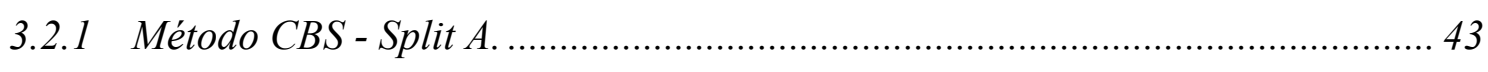

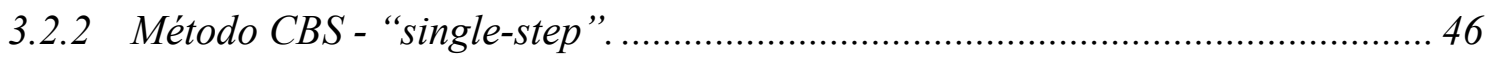

3.3 ESQUEMA HÍBRIDO: TAYLOR-GALERKIN MODIFICADO (MTG: MODIFIED

TAYLOR-GALERKIN). 
3.4 MONTAJE, ACTUALIZACIÓN DE VARIABLES PRIMARIAS Y CÁLCULO DE

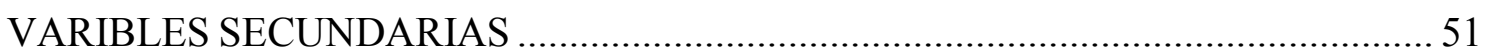

4 INTEGRACIÓN ANALÍTICA DE MATRICES ELEMENTALES...................53

4.1 ELEMENTO ISOPARAMETRICO DE OCHO NODOS ............................................. 53

4.2 TRANSFORMACIÓN DE DOMINIO DE INTEGRACIÓN …..................................... 54

4.3 INTEGRACIÓN ANALÍTICA DE MATRICES ELEMENTALES $1: \boldsymbol{J}(0) \ldots \ldots \ldots \ldots \ldots . . . . . . . . . . . . .56$

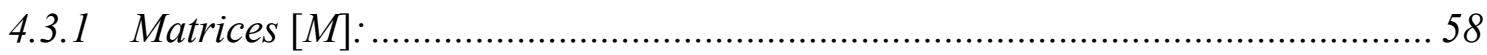

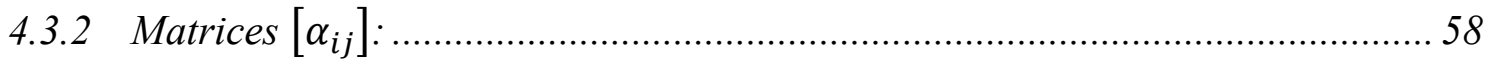

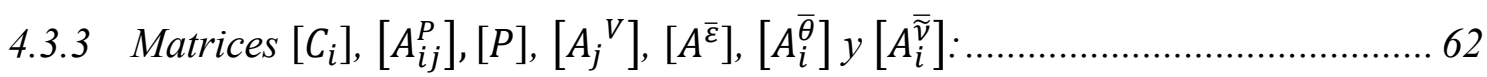

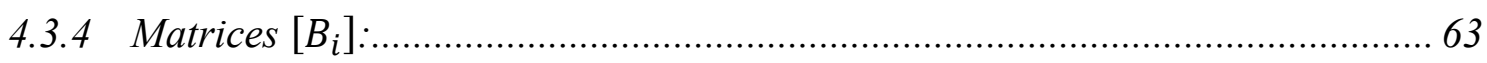

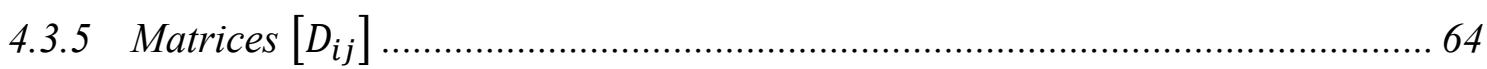

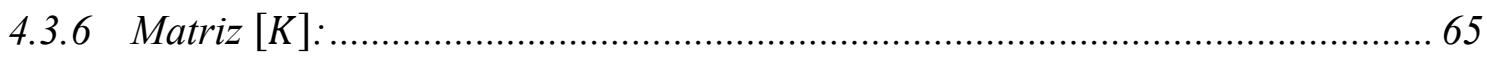

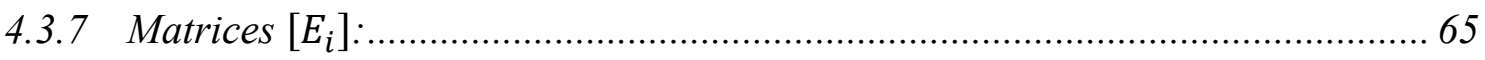

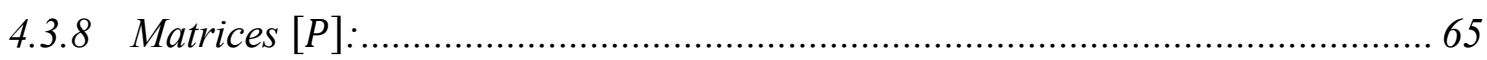

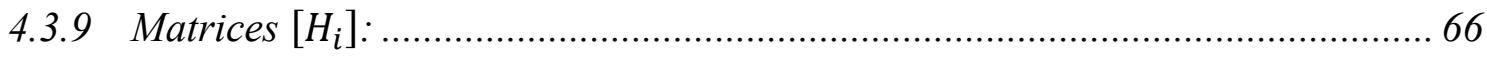

4.4 INTEGRACIÓN ANALÍTICA DE MATRICES ELEMENTALES $2: J\left(\xi_{1}, \xi_{2}, \xi_{3}\right) \ldots \ldots 6$

4.5 TRATAMIENTO DE LAS INTEGRALES DE CONTORNO ..................................... 69

4.6 VECTORES $\{Q\}^{n} \mathrm{Y}\left\{Q v_{i}\right\}^{n}$ EN LA ECUACIÓN DE TRANSPORTE DE SPALART-

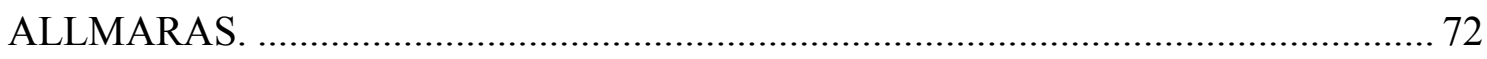

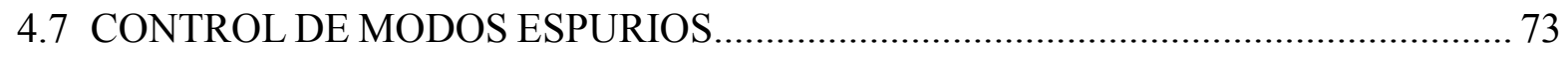

5 ASPECTOS IMPORTANTES DE LA IMPLEMENTACIÓN. ......................74

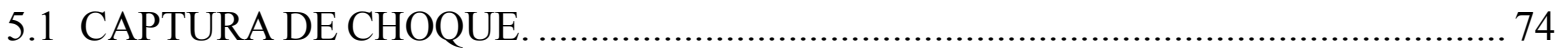

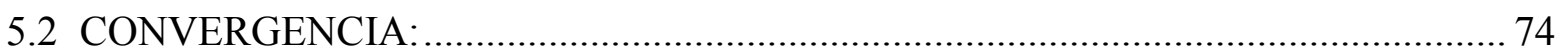

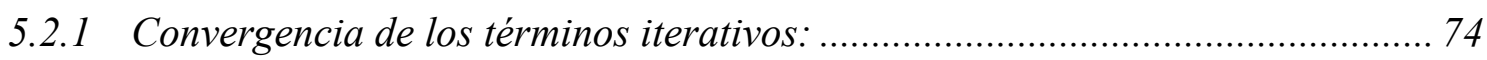

5.2.2 Convergencia a estado estacionario o alcance del tiempo de simulación

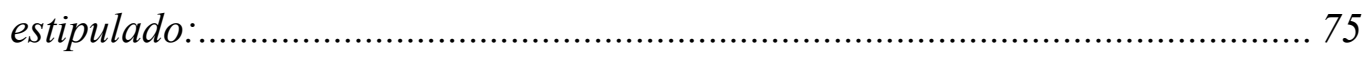

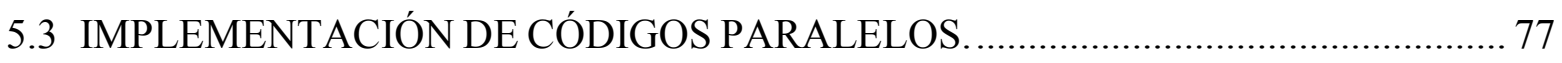

5.3.1 Descripción general de la metodología de paralelización.................................. 77

5.3.2 Métricas de los programas paralelos. .................................................................. 79

5.3.3 Evaluación de eficiencia de paralelización: Flujo supersónico no viscoso alrededor de una esfera .................................................................................. 81

6 PROBLEMAS DE VERIFICACIÓN Y VALIDACIÓN. .................................85

6.1 FLUJO TRANSÓNICO LAMINAR EN TORNO A UN PERFIL AERODINÁMICO. 85 
6.2 FLUJO SUPERSÓNICO TURBULENTO SOBRE PLACA PLANA. 90

6.3 FLUJO HIPERSÓNICO "FRIO” TURBULENTO “ASWBLI”: INTERACCIÓN ENTRE CAPA LÍMITE Y ONDA DE CHOQUE. .95

7 CONCLUSIONES. .........................................................101

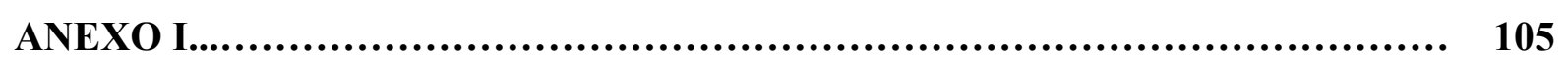

INTRODUCCIÓN............................................................ 105

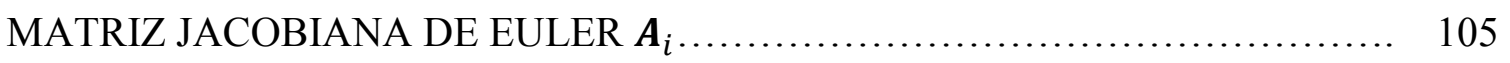

EXPANSIÓN DE LOS TÉRMINOS DE ESTABILIZACIÓN..................... 106

Ecuación de continuidad.................................................... 107

Ecuaciones de conservación de cantidad de movimiento........................ 108

Ecuación de conservación de energía......................................... 110

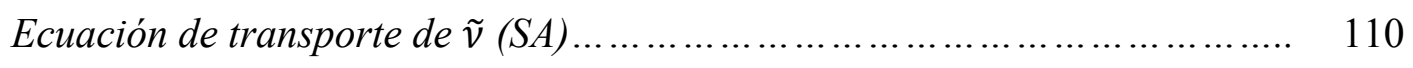

REFERENCIAS............................................................... 112 


\section{INTRODUCCIÓN.}

\subsection{LA DINÁMICA DE FLUIDOS COMPUTACIONAL}

En las industrias aeroespacial, ferroviaria y automotriz se utilizan túneles de viento para medir las características aerodinámicas de los vehículos en la fase de diseño. Aunque estas metodologías experimentales suelen involucrar procesos costosos y extensos en el tiempo, resultan absolutamente necesarias para la determinación de parámetros de diseño.

La fluidodinámica computacional (CFD: Computational Fluid Dynamics) aparece como una herramienta complementaria que, en ocasiones, permite ahorrar costos al reducir la cantidad de ensayos en túnel de viento a un mínimo indispensable, y constituye una herramienta que, correctamente utilizada y validada con técnicas experimentales, permite dar soporte a la toma de decisiones referidas al diseño aerodinámico ${ }^{[1]}$. En algunos casos, las técnicas de CFD permiten acelerar los tiempos de diseño mediante la simulación de condiciones que serían muy difíciles de reproducir en ensayos físicos.

Pero el campo de acción de CFD no se limita a las industrias relativas al transporte, dado que se ha ido extendiendo, y hoy en día hay una gran variedad de usos como, por ejemplo, en el estudio de la acción del viento sobre estructuras civiles, en estudios meteorológicos, médicos, biológicos y la industria petrolera, sólo por mencionar algunos ejemplos.

El objetivo de los ingenieros que trabajan utilizando códigos de CFD es evaluar las principales características de los flujos involucrados en las diferentes áreas de investigación. Por otro lado, el objetivo de los investigadores que desarrollan las técnicas de CFD es plantear y evaluar diferentes algoritmos que permitan simular los diferentes tipos de flujos, alcanzando una amplia gama de problemáticas, y dar a conocer el conocimiento adquirido en el estudio de estos algoritmos para que sean aplicados, o tenidos en cuenta, en los programas de simulación de flujos. Esta tesis se enmarca en este último campo.

Entonces, para que los algoritmos evaluados resulten de utilidad, los mismos deberán ser capaces de determinar los valores de variables como la presión, las componentes de la 
velocidad, la temperatura y la densidad, así como las fuerzas viscosas y de presión que aparecen en el contorno sólido en contacto con el fluido.

Para lograr este objetivo, los algoritmos deben resolver las ecuaciones de la mecánica de fluidos, con balances de masa, de cantidad de movimiento y de energía. En el caso de fluidos newtonianos, luego de la introducción de las ecuaciones constitutivas, las ecuaciones obtenidas para la conservación de la cantidad de movimiento resultan ser las conocidas ecuaciones de Navier-Stokes (NSE: Navier-Stokes Equations) ${ }^{[2]}$.

Pero algunas de las características principales de algunos flujos pueden obtenerse asumiendo que los efectos viscosos y de conducción térmica están confinados en las proximidades de las superficies sólidas, y que el resto del flujo se comporta como si no fuera viscoso. Los flujos en las regiones con poca influencia de la viscosidad se modelan despreciando los términos viscosos de las ecuaciones de balance de cantidad de movimiento, dando como resultado las ecuaciones de Euler. Los términos de conducción térmica son también despreciados en la ecuación de energía, obteniendo un modelo matemático simplificado. Esta simplificación es útil sólo en casos en que las regiones donde están confinados los efectos viscosos son muy próximas a los contornos sólidos y brinda información parcial sobre el flujo. En consecuencia, las ecuaciones de Navier-Stokes siempre son indispensables cuando se desea obtener información sobre la capa límite, estelas de corte y las fuerzas viscosas que actúan sobre los contornos sólidos ${ }^{[3]}$.

Así, circunscribiendo el área de atención a la dinámica de gases newtonianos, los modelos matemáticos utilizados en la simulación numérica de flujos compresibles están formados por la ecuación de continuidad, las NSE y la ecuación de energía, todas expresadas en su forma conservativa. (A este conjunto de ecuaciones también suele llamárselo simplemente como ecuaciones de Navier-Stokes, sobreentendiendo que ya están incluidas la ecuación de continuidad y la ecuación de energía)

La gran mayoría de flujos externos reales presentan números de Reynolds elevados con lo cual el tratamiento de la turbulencia, tanto en capas límite como en estelas de corte, es necesario si se desean evaluar algoritmos para contribuir a la solución de problemas de la ingeniería moderna ${ }^{[4],[5]}$.

Existen diferentes formas de abordar la simulación de flujos turbulentos, que pueden clasificarse de acuerdo con qué escalas de turbulencia se simulan directamente resolviendo las ecuaciones de Navier-Stokes no estacionarias y qué escalas se modelan ${ }^{[6]}$. 
La simulación numérica directa (DNS, por sus siglas en inglés: Direct Navier-Stokes) procura simular todas las escalas de la turbulencia y no utilizar modelo alguno. La resolución de la malla y el paso del tiempo máximo permitido para un cálculo de DNS deben ser lo suficientemente pequeños como para capturar las escalas de Kolmogorov del flujo turbulento. La relación inversa de estas escalas con el número de Reynolds indica que las mallas requeridas deben ser más finas según aumenta el número de Reynolds.

La turbulencia, y por lo tanto la solución DNS, es inherentemente no estacionaria y tridimensional, y el algoritmo numérico utilizado en el proceso de solución DNS debe tener muy baja disipación numérica para permitir que todas las escalas del flujo turbulento sean representadas. Todo esto redunda en la necesidad de utilizar mallas extremadamente finas y muy uniformes. Esto ha hecho que muchos trabajos hechos con DNS se concentren en problemas con números de Reynolds relativamente bajos y geometrías simples.

Para evitar esto, otra forma de tratar la turbulencia es por medio de la resolución de ecuaciones de Navier-Stokes promediadas (RANS, Reynolds Averaged Navier-Stokes, o FANS, Favre Averaged Navier-Stokes). En este enfoque se transforman las ecuaciones de Navier-Stokes en un modelo matemático similar, pero con variables promediadas en el tiempo, de manera que las ecuaciones resultantes sólo ofrecen una solución para las variables promediadas. El enfoque de las RANS (o FANS) utiliza una sola escala para caracterizar el espectro turbulento, como se muestra en la figura $1.1^{[6]}$.

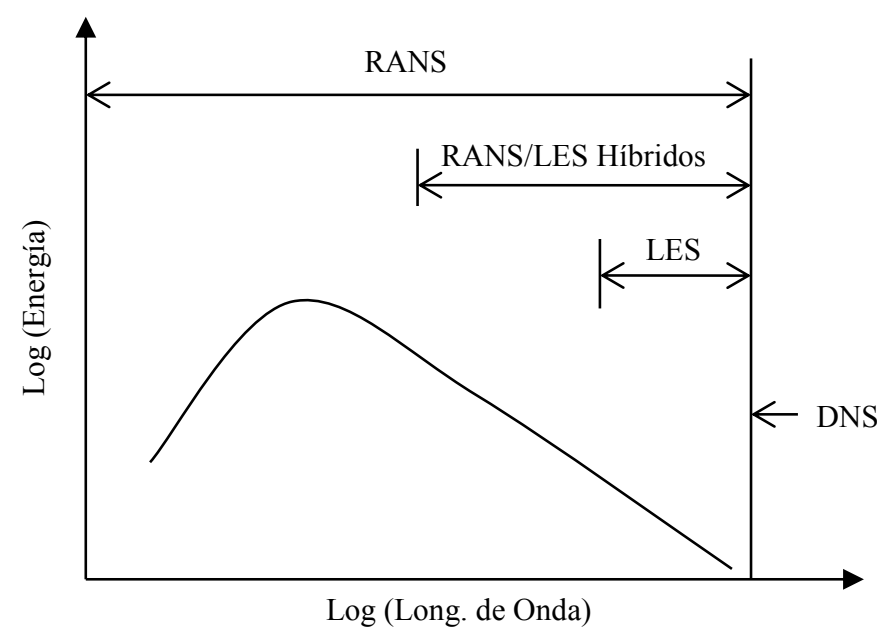

Figura 1.1: Diferentes métodos de simulación de turbulencia según las escalas del espectro turbulento.

Durante el proceso de promediado aparecen nuevos términos que dependen de las fluctuaciones de las variables involucradas, los cuales deben ser modelados para representar los efectos que dichas fluctuaciones tienen sobre el comportamiento del flujo promediado que se pretende obtener. Esto ha dado origen a la aparición de diversos modelos de turbulencia. 
Así, en este enfoque, las propiedades no estacionarias de las fluctuaciones turbulentas no son tenidas en cuenta en la solución. Esto permite el uso de mallas mucho menos refinadas y pasos de tiempo mucho mayores que para una solución DNS. Comparado con la solución DNS, este enfoque permite el uso de algoritmos con mayor grado de disipación numérica. Los modelos de turbulencia desarrollados para cerrar las RANS (o FANS) son ampliamente utilizados en ingeniería por sus relativamente bajos requerimientos en términos de tiempo y poder de cómputo. Sin embargo, los diferentes modelos presentan virtudes y defectos según el caso y el tipo de flujo que se pretende simular. Por otro lado, los efectos no estacionarios de la turbulencia no son tenidos en cuenta, lo cual en muchos casos resulta en perdida de información relevante del problema, dado que la solución obtenida no es la solución de las ecuaciones originales, sino la solución en términos de variables promediadas en el tiempo.

La simulación de grandes vórtices (LES - Large Eddy Simulation) es un enfoque basado sobre la idea de simular las escalas de turbulencia que son mayores que el tamaño de elemento de la malla y modelar sólo las escalas turbulentas sub-malla. Las pequeñas escalas turbulentas pueden considerarse isotrópicas, por lo que son resueltas con modelos de turbulencia bastante simples. Esto se efectúa resolviendo en forma directa las ecuaciones de Navier-Stokes sólo para las escalas que pueden ser captadas por la malla mientras se aplica un filtro espacial y un modelo para las escalas sub-malla.

Como ocurre en el caso de las aplicaciones de DNS, al resolver con LES se debe tener cuidado al elegir un algoritmo numérico que tenga una disipación numérica lo suficientemente baja como para que no haya una disminución en el número de Reynolds efectivo del problema que se pretende simular.

Si bien las aplicaciones de LES permiten simular problemas con números de Reynolds mayores que en el caso DNS con los mismos recursos computacionales, en casos de aplicación industrial generalmente se requiere mallas muy refinadas que necesitan mucho tiempo de cómputo para obtener la solución, cuando se lo compara con el tiempo requerido para efectuar una simulación equivalente con los modelos de turbulencia usando RANS.

En la última década y media ha surgido una nueva clase de modelos de turbulencia para flujos no estacionarios con altos números de Reynolds. Estos modelos de turbulencia se llaman modelos RANS/LES híbridos ${ }^{[7]}$. Estos modelos de turbulencia híbridos son extensiones de los esquemas LES en los que se utilizan modelos de turbulencia RANS modificados como modelos sub-malla para resolver las ecuaciones de Navier-Stokes. 
La meta para la mayoría de estas las aplicaciones es utilizar RANS (o FANS) para el cálculo de la capa límite donde las escalas turbulentas muy pequeñas están presentes y utilizar LES para simular las escalas turbulentas localizadas lejos del cuerpo. Por lo tanto, las estructuras turbulentas de gran escala lejos del cuerpo son simuladas por las ecuaciones de Navier-Stokes no estacionarias. Estos esquemas sirven como un puente entre los modelos tradicionales de RANS y LES y permiten utilizar mallas menos refinadas que con un enfoque LES. Estas técnicas tampoco requieren que el algoritmo seleccionado presente tan baja disipación numérica como en los casos de DNS y LES.

\subsection{DIFERENTES MÉTODOS NUMERICOS.}

Con los años, mientras se desarrollaban las técnicas de CFD, han aparecido varios métodos para transformar al dominio continuo original en un dominio discreto que pueda ser tratado con técnicas de resolución de ecuaciones algebraicas.

El método de diferencias finitas ha sido ampliamente utilizado, por su sencillez y el hecho de ser uno de los primeros métodos desarrollados. Sin embargo, esta técnica en sus orígenes tenía la desventaja de ser difícil de aplicar en mallas irregulares con geometrías complicadas, lo cual llevó a la aparición de otras metodologías. Aun así, el método de diferencias finitas continúa siendo utilizado en muchas áreas de investigación aún hoy ${ }^{[2]}$.

El método de volúmenes finitos (FVM: Finite Volume Method) es muy flexible y aplicable en mallas irregulares. Su gran ventaja consiste en el hecho de que las leyes de conservación, en su forma integral, se llevan a su versión discreta directamente. Por lo tanto, no es necesario recurrir a funciones de interpolación o funciones aproximadoras y no se necesitan métodos para minimizar los errores, como los métodos variacionales o técnicas de los residuos ponderados. Sin embargo, si bien existen algunas aplicaciones de este método a problemas de mecánica del sólido (CSM: Computational Solid Mechanics) ${ }^{[8]}$, las referencias a estas aplicaciones del FVM son relativamente escasas, y su uso en la industria está restringido a los problemas fluidodinámicos y de transferencia de calor, dejando fuera, por ejemplo, los problemas de interacción fluido-estructura que requieren el modelado de estructuras deformables. En estos problemas, la alternativa más frecuentemente implementada es resolver el flujo de fluidos usando el FVM y algún otro método para modelar la estructura, tal como el método de elementos finitos (FEM: Finite Element Method). Esto requiere el traspaso de datos entre ambos modelos, dado que un enfoque monolítico no es posible cuando se utilizan diferentes métodos para modelar la estructura y el fluido. Aun así, es de destacar 
que el FVM constituye la técnica más extendida para la resolución de los más variados tipos de flujos ${ }^{[9]}$.

El FEM es el más general de los métodos descritos en este documento. Ofrece la gran ventaja de ser aplicable a una amplia gama de áreas de ingeniería (sólidos y fluidos) y tiene la posibilidad de usar mallas adaptativas, actualizadas en base al error de la solución aproximada. Esta adaptación puede darse a través del refinamiento automático de la malla o mediante el aumento del orden de las funciones de interpolación [3], [10].

Otra técnica que ha logrado éxito en modelización numérica de problemas de mecánica de fluidos es el método de elementos de contorno (BEM: Boundary Element Method) pero su uso está restringido a algunas áreas de la ingeniería. Estas técnicas no son de aplicación tan general como el FEM o el FVM ${ }^{[11]}$.

Los métodos numéricos como el método de elementos finitos, método de diferencias finitas y método de volúmenes finitos se definieron originalmente en mallas con nodos. En una malla así, cada punto tiene un número fijo de vecinos predefinidos y esta conectividad entre vecinos puede usarse para definir operadores matemáticos. Estos operadores se utilizan para construir las ecuaciones para simular problemas de la mecánica del continuo, tales como las ecuaciones de Euler o las ecuaciones de Navier-Stokes. Pero en algunas simulaciones donde el material que se está simulando posee interfaces móviles o donde pueden ocurrir grandes deformaciones del material (como en simulaciones de problemas de plasticidad), la conectividad de la malla puede ser difícil de mantener. Si la malla se distorsionara demasiado o se degenerara durante la simulación, los operadores definidos en ella ya no pueden dar valores correctos. La malla puede adaptarse durante la simulación (un proceso llamado remallado adaptativo) pero esto tiene un costo computacional que debe considerarse. Una alternativa en esta clase de problemas son los métodos sin malla (Meshless Methods) ${ }^{[12]}$. Los métodos sin malla suelen aplicarse en casos como los siguientes:

- Simulaciones donde los nodos pueden ser creados o destruidos, como en casos de crecimiento de grietas.

- Simulaciones donde plantear geometrías o fronteras que pueden moverse sin corresponderse con una malla representa una ventaja.

- Simulaciones con materiales no lineales, con discontinuidades o singularidades.

En resumen, existen diferentes métodos de simulación, cada uno de los cuales ofrece ventajas comparativas en algunos campos específicos de aplicación. Todos ellos continúan 
siendo utilizados e investigados con el fin de mejorar la calidad de los resultados y la performance de los algoritmos construidos con base en dichos métodos.

\subsection{LÍNEA DE INVESTIGACIÓN DE ESTA TESIS}

\subsubsection{Desarrollo de métodos numéricos}

Esta tesis se enfoca en la simulación numérica de flujos de gases compresibles utilizando el método de los elementos finitos, por tratarse de un método de aplicación muy general y por tratarse de la línea histórica de investigación del tesista. La selección del método permitiría, en caso de continuar desarrollando esta línea en el futuro, plantear un enfoque monolítico para problemas de interacción fluido-estructura.

En problemas fuertemente convectivos, como en el caso de los flujos compresibles, el método estándar de Galerkin conduce a procedimientos numéricos cuyos resultados presentan oscilaciones espurias e inestabilidades. Inicialmente estos contratiempos fueron resueltos por la introducción de funciones de interpolación modificadas, método conocido como formulación de Petrov-Galerkin, el cual fue introducido por Christie et al. ${ }^{[13]}$. Este método resultó adecuado para la resolución en una dimensión, pero su extensión a problemas multidimensionales no resultó satisfactoria.

Más tarde, Hughes [14] introdujo el método SPUG ("Streamline Upwind Petrov Galerkin”) cuya idea básica es utilizar funciones de peso adicionando perturbaciones en la dirección de las líneas de corriente, es decir, en la dirección del flujo. Sin embargo, esta técnica se desarrolló para las formulaciones de estado estacionario, y su aplicación en problemas no estacionarios no resultó adecuada.

El método de Taylor-Galerkin fue introducido por Donea ${ }^{[15]}$ y consiste en una expansión de la serie de Taylor en el tiempo aplicando luego el método de Galerkin para la discretización en el espacio. Esta formulación es una extensión de las técnicas de diferencias finitas de orden superior del tipo Lax-Wendroff a las formulaciones débiles válidas en aproximaciones de elementos finitos, y ha demostrado ser satisfactoria en la simulación de flujos altamente compresibles, pero los resultados decaen en calidad a medida que el grado de compresibilidad disminuye. En regímenes subsónicos compresibles para bajos números de Mach $(\mathrm{M}<0,9)$, o en flujos casi incompresibles, esta metodología sufre deficiencias. Los resultados obtenidos utilizando el esquema explícito TG de un paso para flujos subsónicos sobre perfiles alares con números de Mach entre 0,5 y 0,85 muestran convergencia lenta y resultados de presión, temperatura y densidad oscilatorios (ver Burbridge ${ }^{[16]}$ ). 
Para abordar este problema se desarrollaron varias técnicas de estabilización. En particular, el algoritmo Characteristic Based Split (CBS) fue desarrollado por algunos investigadores, tales como Zienkiewicz y Codina ${ }^{[17]}$, con la intención de obtener un único algoritmo que presentara adecuado comportamiento, tanto en regímenes subsónicos como supersónicos. Durante las dos últimas décadas este algoritmo se estableció como una herramienta reconocidamente útil para el cómputo de un amplio espectro de flujos incompresibles o compresibles a diferentes números de Mach (ver Massarotti et al. [18]; Boonmarlert et al., $\left.{ }^{[19]}\right)$. En particular, en el trabajo presentado por Zienkiewicz et al. ${ }^{[20]}$ se muestra la forma explícita del algoritmo y su desempeño para flujos subsónicos, transónicos y supersónicos.

Desde su aparición el método CBS ha ido extendiéndose en su aplicación y puede encontrase una interesante gama de problemas resueltos con este método. A modo de ejemplo se mencionan un trabajo de L. Zhang, et al. donde se muestra una aplicación de este algoritmo para la simulación de flujos en reservorios de petróleo ${ }^{[21]}$ y un trabajo de P. Kopp donde el método CBS se utiliza para la simulación de aguas someras (shallow water) ${ }^{[22]}$.

Sin embargo, es importante tener en cuenta que una de las características que permiten al CBS mostrar una convergencia mejorada es el uso de pasos de tiempo internos en la formulación de las matrices elementales (ver Nithiarasu \& Zienkiewicz ${ }^{[23]}$ ), lo cual también debería ser aplicado al esquema TG para tener una adecuada comparación. El autor de este trabajo no encontró referencias de aplicaciones del esquema TG implementados con paso de tiempo interior.

También es importante mencionar que esta ventaja comparativa del método CBS en cuanto a sus características de convergencia es a costo de tiempo de simulación, debido a la operación de "Split" típica del algoritmo CBS. Los desarrolladores de esta técnica mencionan que esta pérdida de tiempo es poco significativa, sin embargo, no aportan evidencias cuantitativas de cuál es el verdadero costo en tiempo de cómputo ${ }^{[17]}$. Quedan entonces sin respuesta, las siguientes incógnitas: ¿Puede mejorarse la convergencia del método TG usando pasos de tiempo interior? ¿Cuál es la magnitud del costo en tiempo de cómputo del Split realizado en el método CBS cuando se lo compara con el esquema de TG? ¿Puede desarrollarse alguna solución que presente las ventajas de ambos esquemas: mejor convergencia sin costo adicional en términos de tiempo de cómputo?

La selección de la formulación de elemento es siempre una cuestión fundamental cuando se trabaja con el FEM. Las matrices elementales deben ser integradas para poder 
formular las ecuaciones de elemento que luego serán ensambladas para obtener un sistema global. Para esto, las técnicas de integración numérica, como por ejemplo las del tipo GaussLegendre, son comúnmente aplicadas en los puntos de integración del elemento. Aquí es donde la formulación de elemento juega un rol clave, que tendrá impacto tanto en la calidad de los resultados como en la eficiencia computacional del código. Con el fin de aliviar el esfuerzo computacional requerido por los métodos de integración numérica algunos autores como Gresho et al. ${ }^{[24]}$ y Kawamoto \& Tanahashi ${ }^{[25]}$ utilizan esquemas de integración con un único punto de integración, o esquemas analíticos, para la solución de flujos incompresibles. De la misma forma, Molina \& Huot ${ }^{[26]}$ lo hacen con flujos compresibles no viscosos.

En el marco de la maestría en Ingeniería de la Universidade Federal de Rio Grande do Sul (PPGEC/UFRGS) Santos ${ }^{[27]}$ realizó algunas aplicaciones del método de elementos finitos para la solución numérica de flujos de fluidos compresibles. El elemento hexaédrico isoparamétrico de ocho nodos y su integración a través de expresiones analíticas fue utilizado por Azevedo ${ }^{[28]}$ en el contexto de la solución de problemas de interacción fluido-estructura con fluidos viscosos incompresibles.

En particular, Burbridge y Awruch ${ }^{[29]}$ utilizaron elementos finitos hexaédricos con evaluación analítica de las matrices elementales, en conjunto con una técnica de captura de choque, para la solución de flujos aerodinámicos compresibles, viscosos laminares y no viscosos. El autor de este trabajo no ha encontrado bibliografía que haga referencia a implementaciones de estas técnicas de evaluación de matrices elementales aplicadas a flujos turbulentos en el contexto del FEM.

Existen trabajos recientes que muestran el desempeño del algoritmo CBS para simular flujos turbulentos (integración numérica). En el texto de Zienkiewicz, Taylor y Nithiarasu ${ }^{[3]}$ se dedica un capítulo entero a esta temática. Sin embargo, todos los ejemplos allí mostrados corresponden a casos de flujo incompresible, o con relativamente bajos números de Mach. La tesis de doctorado de Chun-Bin Liu ${ }^{[30]}$, muestra la aplicación del método CBS en este contexto de flujos incompresibles.

En el trabajo de Z. Han, et al. ${ }^{[31]}$ se utiliza el algoritmo CBS en conjunto con el modelo de turbulencia de Spalart-Allmaras (SA) para simular un flujo incompresible alrededor de cilindros de base cuadrada, mostrando una aplicación de esta combinación de técnicas a la ingeniería oceánica. Otra implementación del algoritmo CBS en conjunto con el modelo de turbulencia SA puede encontrarse en el trabajo de M. Scungio et al. ${ }^{[32]}$ Aquí se muestra una aplicación a la simulación de dispersión de partículas ultrafinas en los encajonamientos 
urbanos. Estos trabajos muestran la actualidad de las aplicaciones de algoritmo CBS en conjunto con el modelo de SA para la simulación de flujos incompresibles turbulentos.

En un contexto diferente, utilizando otros algoritmos y esquemas numéricos, el modelo de turbulencia de Spalart-Allmaras ha sido aplicado a una amplia gama de problemas, y existen varias formas de este modelo, muchas de ellas propuestas para diferentes condiciones de flujo ${ }^{[33]}$. Algunas aplicaciones recientes de este modelo muestran su utilidad y actualidad específicamente para la simulación de flujos aerodinámicos externos turbulentos ${ }^{\text {[34], [35], [36]. }}$.

\subsubsection{Antecedentes}

En el marco de la maestría en Ingeniería de la Universidade Federal de Rio Grande do Sul (PPGEC/UFRGS) fue desarrollado un trabajo implementando un algoritmo para simular flujos compresibles tridimensionales de fluidos viscosos y no viscosos (ver H. P. Burbridge ${ }^{[16]}$ ). Se utilizó el esquema explícito de un paso de Taylor-Galerkin, implementado en un código computacional propio programado en el lenguaje FORTRAN 90. El objetivo de dicho trabajo era evaluar el desempeño del esquema de Taylor-Galerkin cuando se lo utiliza con elementos hexaédricos isoparamétricos con integración analítica, por lo cual resultó indispensable hacerlo mediante la implementación de un código propio.

Dado que no fue implementado ningún modelo de turbulencia, los casos viscosos evaluados se limitaban a bajos números de Reynolds, constituyendo principalmente casos conocidos en la literatura por ser casos típicos para evaluar el desempeño de los algoritmos.

Aunque aquel estudio no abordó el análisis de problemas de interacción fluido estructura, se contempló la implementación de rutinas que utilizaban tanto una descripción euleriana como una descripción Arbitraria Lagrangeana Euleriana (ALE), con miras a una futura implementación monolítica para problemas de interacción fluido estructura.

Las características esenciales de la implementación numérica eran las siguientes:

- Discretización en el tiempo siguiendo una serie de Taylor;

- Discretización espacial mediante el FEM con el esquema clásico de Bubnov-Galerkin;

- Formulación de elementos hexaédricos trilineales de ocho nodos;

- Integración analítica de las matrices elementales (un punto de integración en el centro del elemento) con control de modos espurios;

- Utilización de un método de captura de choque por medio del agregado de viscosidad artificial en forma selectiva, sólo en aquellas regiones del dominio donde aparecen importantes gradientes de presión (ondas de choque). 
- Codificación en FORTRAN 90 y vectorización para su uso en la supercomputadora CRAY T94 del Centro Nacional de Supercomputação (CESUP) de la región sur, que pertenecía a la Universidade Federal de Rio Grande do Sul (UFRGS).

Partiendo de los códigos iterativos ya desarrollados para correr en procesadores escalares y vectoriales durante aquella tesis, los mismos fueron paralelizados para poder correr tanto en ambiente de memoria compartida (computadora con más de un procesador o núcleo de procesamiento para la cual todos los núcleos acceden al mismo banco de memoria RAM) como en ambiente de memoria distribuida ("clusters" de varias computadoras conectadas en red, cada una de las cuales puede tener uno o más procesadores, a su vez, con uno o más núcleos de procesamiento). Este trabajo de paralelización consistió en la tesis de doctorado de J. R. Masuero ${ }^{[37]}$.

Para ello se optó por una programación paralela que pudiese ser utilizada con una mínima dependencia del sistema operativo (SO) o de la estructura del hardware. Así, toda la lógica de distribución de tareas, balanceo de cargas de trabajo y organización de la comunicación de los datos entre las computadoras está implementada directamente en el código, sin ninguna otra capa de software de gerenciamiento.

Para el uso de múltiples procesadores o núcleos en una única computadora en ambiente de memoria compartida, el soporte es provisto por los compiladores también a través de la API MPI, exigiendo del sistema operativo apenas el soporte de multiprocesamiento.

La división de tareas utilizada empleó el particionado nodal de la estructura de datos basado en el presupuesto de que el esfuerzo computacional asociado a cada nodo de la malla es constante, y también basado en un indicador inicial de la capacidad de procesamiento de cada procesador como, por ejemplo, la frecuencia del procesador. La tarea de división de la malla según su menor dimensión geométrica (para definir las cargas de trabajo de cada procesador) fue realizada mediante la división del sistema de ecuaciones según su menor dimensión (ancho de banda). Para esto, se utilizó un tratamiento de malla basado en una única reordenación nodal para minimización del ancho de banda de la matriz del sistema de ecuaciones. Este tratamiento puede ser considerado como una variante del método global de particionado en fajas (stripwise partitioning - STRIP) (ver Dorneles ${ }^{[38]}$ ). Se emplean elementos fantasma (ghost elements) para minimizar la comunicación entre procesadores a costa de redundancia en el esfuerzo computacional (ver Demkowicz et al. ${ }^{[39]}$ ).

Todos los códigos paralelos fueron implementados en FORTRAN 90. La comunicación de datos entre computadoras se hace utilizando las librerías MPICH de acceso público. Los 
códigos fueron originalmente compilados para ejecutarse en el SO Windows XP, únicamente por ser el sistema operativo utilizado en el laboratorio de computación del CEMACOM/UFRGS. Como la única exigencia con relación al sistema operativo es el soporte a la comunicación en red y al API MPI, y dado que tanto FORTRAN 90 como MPI tienen patrones que garantizan su portabilidad, los códigos desarrollados pueden ser utilizados en otras plataformas y otros sistemas operativos. Así, posteriormente, estos mismos códigos fueron compilados y corridos en Windows 7 y en las distribuciones Debian 9 "Stretch" y Red Hat 6.3 de Linux (trabajo realizado en el CEDI de la UTN FRH).

\subsection{OBJETIVOS.}

Como continuidad de esta línea de investigación, son objetivos prioritarios de esta tesis (los que también constituyen el aporte original de la misma) los siguientes:

1) Evaluar el desempeño de los algoritmos de Taylor-Galerkin y CBS cuando se los implementa con elementos hexaédricos isoparamétricos de ocho nodos con integración analítica de matrices elementales. Comparar sus desempeños y verificar las supuestas ventajas de convergencia del método CBS frente al esquema TG. Realizar estas tareas para flujos no viscosos, viscosos laminares y viscosos turbulentos, incorporando el modelo de Spalart-Allmaras para ver su desempeño cuando se lo utiliza en este contexto (FEM-TG-CBS-Integración analítica-Flujo compresible supersónico).

Aquí interesa la comparación de la calidad de resultados entre ambos esquemas y convergencia y el análisis de metodologías de estabilización para regímenes transónicos. Como fuera expresado anteriormente, una de las características que permiten al CBS mostrar una convergencia mejorada es el uso de pasos de tiempo internos en la formulación de las matrices elementales. Sin embargo, según entiende el autor de este trabajo, al comienzo del mismo esta técnica de estabilización no había sido probada aún con el esquema TG. En este trabajo se implementan pasos de tiempo interiores en el algoritmo de Taylor-Galerkin explorando su desempeño, con la intención de determinar si también el esquema explícito TG puede ser estabilizado mediante el uso de estos pasos de tiempo interiores o no.

Interesa también analizar alternativas de integración analítica, por ejemplo, utilizando herramientas de resolución simbólica (MAXIMA) para integrar algunas de las matrices elementales (interesan principalmente las matrices convectivas), de manera que puedan integrarse en forma completa sin adoptar valores constantes de la matriz Jacobiana y su determinante en el centro del elemento. 
Con respecto a la simulación de la turbulencia el foco está puesto en la resolución de flujos de capa límite (Wall bounded Flow) turbulentos resolviendo las ecuaciones FANS (Favre Averaged Navier-Stokes) con el modelo de Spalart-Allmaras. Al comienzo de este trabajo no existían, según el mejor entendimiento del autor, trabajos que combinen los algoritmos explícitos de Taylor-Galerkin y CBS utilizados para resolver problemas turbulentos compresibles con altos números de Mach con la utilización de elementos hexaédricos con integración analítica y el modelo de turbulencia de Spalart-Allmaras. Es de interés del autor evaluar el comportamiento de estos esquemas en este contexto particular, con la combinación de técnicas recién mencionada. Especialmente interesa ver el desempeño de la integración analítica de matrices elementales con las mallas utilizadas para flujo viscoso externo turbulento compresible, las cuales pueden presentar relaciones de aspecto elevadas.

También es importante mencionar que aquí se utiliza una variante conservativa de la ecuación de transporte de Spalart-Allmaras, de uso poco frecuente, que fuera sugerida por Spalart y Allmaras ${ }^{[40]}$ como una alternativa para flujos compresibles.

2) Realizar análisis comparativo de los tiempos de cómputo del esquema TG y el método $C B S y$, eventualmente, proponer cambios en estos esquemas intentando mejorar las características en términos de tiempo de cálculo y convergencia.

Aquí el foco está puesto en dar respuesta a las siguientes incógnitas planteadas anteriormente: ¿Cuál es la magnitud del costo en tiempo de cómputo del Split realizado en el método CBS cuando se lo compara con el esquema de TG? En caso de que existan diferencias apreciables: ¿Puede desarrollarse alguna solución que presente las ventajas de ambos esquemas, es decir, mejor convergencia sin costo adicional en términos de tiempo de cómputo?

3) Elaborar códigos propios que, eventualmente, puedan ser utilizados en cursos de posgrado y cursos de grado para impartir clases y que puedan servir de base a investigaciones futuras sobre algoritmos especificos de cálculo de problemas aerodinámicos y de interacción fluido-estructura.

Compartir con la comunidad académica los códigos implementados y los conocimientos adquiridos durante el desarrollo de esta tesis es uno de los objetivos propuestos. El tesista desarrolla actividades como profesor adjunto de Mecánica de Fluidos de la Universidad Tecnológica Nacional, Facultad Regional General Pacheco (UTN-FRGP), y a partir de 2016, también en el Centro de Estudios De Informática (CEDI) de la Universidad Tecnológica Nacional, Facultad Regional Haedo (UTN-FRH), en el que colaboran el señor 
Andrés Trapanotto y el ingeniero Juan Carlos Polidoro, con foco en la preparación de los códigos, previamente paralelizados, para ser compilados y ejecutados en clusters con SO Linux (distribución Debian) y otras tareas relativas al preprocesamiento de datos.

En particular, este último grupo tiene como misión derramar los desarrollos y resultados de sus actividades al resto de la comunidad académica de la Facultad Regional Haedo, por lo cual este objetivo es compartido y concurrente con esta tesis.

\subsection{RECUSOS DISPONIBLES Y MEDIOS DE FINANCIAMIENTO.}

El presente trabajo se enmarca en los siguientes proyectos:

- UNLP 11/I141 “Diseño conceptual de vehículo lanzador”, desde el 01/01/2010 hasta el $31 / 12 / 2013$

- UNLP I198 "Ingeniería de un vehículo lanzador experimental" Proyecto de Incentivos. 01/01/2014-31/12/2017

- SNCAD 2012: "Mejoramiento de la conectividad y ampliación de la capacidad de cómputo existente en el GFC/GEMA". Sistema Nacional de Computación de Alto Desempeño, SNCAD

- Proyecto de desarrollo de códigos e implementación de algoritmos de simulación numérica del Centro de Estudios De Informática (CEDI) de la Universidad Tecnológica Nacional, Facultad Regional Haedo (UTN-FRH).

Durante el desarrollo de este trabajo se utilizaron los siguientes recursos informáticos:

- Una computadora Desktop con cuatro núcleos de procesamiento y $8 \mathrm{~Gb}$ de memoria RAM y SO Windows 7 de propiedad de tesista. (Esta máquina fue utilizada para escritura de los códigos y compilación en plataforma Windows 7, y ejecución paralela hasta con cuatro hilos (threads) de problemas de pequeño porte).

- Un cluster tipo Beowulf no homogéneo con sistema operativo Linux (Debian 9 "Stretch") propiedad del CEDI UTN-FRH con seis nodos con cuatro núcleos de procesamiento y $4 \mathrm{~Gb}$ de memoria RAM cada uno más seis nodos de un núcleo de procesamiento y $1 \mathrm{~Gb}$ de memoria RAM cada uno. (Este cluster no homogéneo es ideal para preparación y test de códigos y análisis de las técnicas de balanceo de carga para la paralelización).

- Un cluster Linux (Red Hat) de la UIDET Grupo Fluidodinámica Computacional (GFC) de la Facultad de Ingeniería de la UNLP con una capacidad de cómputo de 96 núcleos de procesamiento y 300 GB de memoria RAM en total. (Este cluster tiene 
potencia de cálculo suficiente como para simular en problemas mayores con los códigos ya configurados en el cluster del CEDI UTN-FRH).

\subsection{CONTRIBUCIONES DE LA TESIS.}

\subsubsection{Contribuciones al avance científico y tecnológico.}

Existen en la industria del software gran cantidad de paquetes comerciales y de código abierto para simulación de CFD (Computational Fluid Dynamics) que pueden abordar de manera muy eficiente el tipo de problemas de flujo que se abordan en esta tesis. Sin embargo, la mayoría de estos códigos están desarrollados con el método de volúmenes finitos (Fluent, CFX, StarCCM+, OpenFOAM). Algunos pocos paquetes están basados en técnicas de elementos finitos, pero no abordan flujos compresibles (Acusim, Acusolve). El software COMSOL, basado en elementos finitos, puede abordar flujos como los que se analizan en este esta tesis.

Si bien lo antedicho nos muestra una amplia gama de opciones para abordar la problemática de flujos aerodinámicos compresibles turbulentos, la opción de desarrollar un código propio ofrece la alternativa de poder evaluar los diferentes algoritmos que existen para resolver estos problemas cuando se utiliza el método de elementos finitos. Esto resulta interesante para proponer mejoras a estos algoritmos y, eventualmente, conseguir mejorarlos para hacerlos más eficientes. De manera que, como ya fue expuesto a lo largo de todo este documento, el objetivo principal es la investigación sobre los algoritmos y su eventual mejora, para lo cual es condición necesaria la utilización de códigos propios.

De esta forma, el estudio (pasado, presente y futuro) de potenciales mejoras a los algoritmos existentes basados en técnicas de elementos finitos es la principal contribución de esta tesis al avance científico y tecnológico.

Como parte del desarrollo de este trabajo se realizó la publicación de un artículo en revista con referato, (ver Burbridge ${ }^{[59]}$ ), y varios artículos en congresos nacionales (por ejemplo, referencias ${ }^{[52]}{ }^{[58]}$ entre otros que no se listan en las referencias). Asimismo, durante el trabajo previo de maestría ya se había publicado un artículo en revista con referato (ver referencia ${ }^{[29]}$ ) y varios trabajos en congresos nacionales e internacionales que no fueron incluidos en las referencias. 


\subsubsection{Contribuciones a la formación de recursos humanos.}

Desde este punto de vista, esta tesis se propuso contribuir de diferentes maneras. El desarrollo de software propio permitió desarrollar recursos humanos con especialización en problemas de fluidodinámica, algoritmos numéricos de solución, métodos de programación y formas de implementación de los algoritmos, paralelización y cómputo de alto desempeño.

Por otro lado, como fuera anteriormente mencionado, si se sistematizan y simplifican los procesos de generación de datos (preprocesamiento) y la integración de los códigos dentro de interfaces graficas existentes, se podrá integrar la utilización de estos códigos en el dictado de materias como Mecánica de Fluidos o Aerodinámica (Por ejemplo, en las carreras de Ingeniería Mecánica e Ingeniería Aeronáutica de la UNLP, o en las Facultades Regional Haedo y Regional General Pacheco de la UTN). Eventualmente, esto podrá extenderse a otras facultades regionales o en cursos de posgrado en los cuales pudiera resultar de utilidad.

\subsection{ORGANIZACIÓN DEL TRABAJO.}

El texto de esta tesis está organizado de la siguiente forma:

En el presente capítulo se da una introducción con mención del estado del arte, situando al lector en contexto y brindando una perspectiva cronológica del desarrollo de los algoritmos existentes para simular flujos compresibles con el método de elementos finitos. También se mencionan las formas de abordar la simulación de la turbulencia y se indica el trabajo de paralelización realizado sobre los códigos. Luego, se describen los objetivos de esta tesis y los recursos disponibles, y se mencionan las contribuciones de este trabajo y su continuación en el ámbito del Centro de Estudios de Informática (CEDI) de la UTN-FRH.

En el capítulo dos se dan detalles de las ecuaciones de gobierno, se desarrolla el modelo de turbulencia con énfasis en el promediado de Favre y la aproximación de Boussinesq. El capítulo finaliza con una descripción, en forma conservativa vectorial compacta, del modelo matemático completo a ser resuelto.

En el capítulo tres se hace una extensiva descripción de los algoritmos y esquemas de Taylor-Galerkin, CBS y CBS de un paso. En este capítulo se introduce, también, el esquema hibrido de Taylor-Galerkin modificado, que es uno de los aportes novedosos de este trabajo.

El capítulo cuatro trata de los métodos de integración de las matrices, a nivel de elemento, que se utilizan en esta investigación. Aquí se indican los dos abordajes utilizados, incluyendo la simplificación que consiste en adoptar la matriz Jacobiana de la transformación 
en el punto central del elemento y las técnicas utilizadas de integración con software de cálculo simbólico.

En el capítulo cinco se abordan temas importantes de la implementación, como ser los métodos de captura de choque (flujo compresible), la forma de calcular los residuos y la convergencia y, especialmente, algunos detalles sobre la paralelización de los códigos que incluyen las métricas obtenidas mostrando la eficiencia de esta implementación paralela.

En el capítulo seis se muestran tres problemas de validación y verificación, en donde se comparan los resultados obtenidos en este trabajo con los mostrados por las referencias y los que se obtuvieron utilizando software comercial reconocido (ANSYS/Fluent 17.1). Pero, fundamentalmente, estos ejemplos sirven para mostrar la eficiencia relativa de los algoritmos implementados, mostrando las características ventajosas de el esquema de Taylor-Galerkin modificado propuesto en este trabajo.

En el capitulo 7 se mencionan las conclusiones del trabajo, indicando la aptitud de los algoritmos para la paralelización, las observaciones respecto del comportamiento de la implementación del modelo de turbulencia y las características en términos de eficiencia y convergencia de los diferentes esquemas y algoritmos estudiados. También se indican las líneas de trabajo futuro a través del Proyecto de Investigación (PID) de la UTN FRH en el marco de los trabajos realizados en el CEDI.

Por último, se agregan un anexo referido al tratamiento de las matrices Jacobianas de Euler que aparecen en el esquema de Taylor-Galerkin y las referencias. 


\section{ECUACIONES DE GOBIERNO CON MODELO DE TURBULENCIA.}

\subsection{ECUACIONES DE CONSERVACIÓN}

Las ecuaciones que rigen el escurrimiento de un fluido viscoso vienen dadas por las siguientes expresiones:

Ecuación de conservación de masa:

$$
\frac{\partial \rho}{\partial t}+\frac{\partial \rho v_{i}}{\partial x_{i}}=0
$$

Ecuaciones de conservación de la cantidad de movimiento:

$$
\frac{\partial\left(\rho v_{j}\right)}{\partial t}+\frac{\partial\left(\rho v_{j} v_{i}\right)}{\partial x_{i}}-\frac{\partial \sigma_{i j}}{\partial x_{i}}=0
$$

Ecuación de conservación de energía:

$$
\frac{\partial(\rho e)}{\partial t}+\frac{\partial\left(\rho e v_{i}\right)}{\partial x_{i}}-\frac{\partial}{\partial x_{i}}\left(v_{j} \sigma_{i j}\right)+\frac{\partial q_{i}}{\partial x_{i}}=0
$$

todas ellas son válidas en el dominio $\Omega$, sin términos fuente, y en todos los casos $i, j=1,2,3$.

En las expresiones anteriores se tiene:

$\checkmark \quad v_{i}$ son las componentes de velocidad,

$\checkmark \quad \rho$ es la densidad,

$\checkmark \sigma_{i j}=-p \delta_{i j}+\tau_{i j}$ son las componentes del tensor de tensiones de Cauchy,

$\checkmark \quad \tau_{i j}$ son las componentes viscosas del tensor de tensiones,

$\checkmark \quad p$ es la presión termodinámica,

$\checkmark \quad \delta_{i j}$ es la delta de Kronecker,

$\checkmark \quad e$ es la energía total específica,

$\checkmark q_{i}$ son las componentes del vector flujo de calor por conducción,

$\checkmark \quad x_{i}$ y $t$ son las coordenadas espaciales y temporal, respectivamente. 


\subsection{RELACIONES CONSTITUTIVAS: FLUIDO NEWTONIANO.}

Para un fluido Newtoniano las componentes de tensión viscosa vienen dadas por

$$
\tau_{i j}=2 \mu\left[S_{i j}-\frac{1}{3} \frac{\partial v_{k}}{\partial x_{k}} \delta_{i j}\right] ; \quad S_{i j}=\frac{1}{2}\left[\frac{\partial v_{i}}{\partial x_{j}}+\frac{\partial v_{j}}{\partial x_{i}}\right]
$$

donde $\mu$ es la viscosidad y el subíndice $k$ corre de 1 a 3 .

Las componentes del vector de flujo de calor por conducción son:

$$
q_{i}=-K \frac{\partial T}{\partial x_{i}}
$$

donde $K$ es la conductividad térmica, $T$ es la temperatura y el subíndice $i$ corre de 1 a 3 .

\subsection{ECUACIONES COMPLEMENTARIAS (AIRE).}

Deben considerarse la ecuación de estado, la relación entre las energías específicas involucradas y una ley de variación de la viscosidad y el coeficiente de conductividad térmica con la temperatura.

La ecuación de estado de los gases perfectos (aire) es la siguiente:

$$
p=(\gamma-1) \rho u
$$

La relación de calores específicos a presión y volumen constantes, $\gamma$, es un número adimensional y, para el aire, resulta: $\gamma=1,4$. La energía interna específica $u$ y la temperatura se relacionan con las variables de campo independientes por la siguiente expresión:

$$
u=c_{v} T=e-\frac{1}{2} v_{i} v_{i}
$$

donde $T$ es la temperatura y $c_{v}$ es el coeficiente de calor específico a volumen constante.

La ley de Sutherland se utiliza en este trabajo para establecer la dependencia de la viscosidad y el coeficiente de la conductividad térmica con respecto a la temperatura. Esta ley puede expresarse de la forma siguiente:

$$
\mu=\mu_{\text {ref }} \frac{S+T_{\text {ref }}}{S+T}\left(\frac{T}{T_{r e f}}\right)^{\frac{3}{2}} ; \quad K=K_{r e f} \frac{S_{K}+T_{r e f}}{S_{K}+T}\left(\frac{T}{T_{r e f}}\right)^{\frac{3}{2}}
$$

Los valores de $S$ y $S_{K}$ pueden encontrarse en White (1974) ${ }^{[41]}$ para la ley de Sutherland basada en temperatura ( $S=110,4 \mathrm{~K}$ y $S_{K}=194 \mathrm{~K}$ para el aire). En las expresiones anteriores, $T_{r e f}$ es una temperatura de referencia, para la cual se conocen los valores de referencia $\mu_{r e f}$ y $K_{r e f}$. 


\subsection{ADIMENSIONALIZACIÓN.}

Como se mencionó anteriormente, todas las expresiones indicadas en este capítulo hasta aquí fueron adimensionalizadas. Las cantidades dimensionales utilizadas en la adimensionalización de las ecuaciones se definen de la siguiente manera:

$$
\begin{array}{ll}
\checkmark t=t^{*} \frac{c_{\infty}{ }^{*}}{L_{r e f}{ }^{*}} & \text { es el tiempo adimensional, } \\
\checkmark \quad x_{i}=\frac{x_{i}^{*}}{L_{r e f^{*}}} & \text { son las coordenadas espaciales adimensionales, } \\
\checkmark \quad v_{i}=\frac{v_{i}^{*}}{c_{\infty}{ }^{*}} & \text { son las componentes adimensionales de velocidad, } \\
\checkmark \quad \rho=\frac{\rho^{*}}{\rho_{\infty}{ }^{*}} & \text { es la densidad adimensional, } \\
\checkmark \quad p=\frac{p^{*}}{\rho_{\infty} c_{\infty}{ }^{2}} & \text { es la presión adimensional, } \\
\checkmark \quad e=\frac{e^{*}}{c_{\infty}{ }^{* 2}} & \text { es la energía total específica adimensional, } \\
\checkmark u=\frac{c_{v}{ }^{*} T^{*}}{c_{\infty}{ }^{* 2}} & \text { es la energía interna específica adimensional, }
\end{array}
$$

donde $L_{\text {ref }}{ }^{*}$ es una longitud de referencia, $c_{\infty}{ }^{*}$ y $\rho_{\infty}{ }^{*}$ son la velocidad del sonido y la densidad de la corriente libre no perturbada. En las anteriores expresiones se utiliza el superíndice asterisco $(*)$ para indicar que la cantidad es dimensional. De aquí en más, las variables y parámetros que no se indiquen con este superíndice asterisco serán cantidades adimensionales. Los términos que contienen temperaturas quedan expresados en función de la energía interna específica adimensional indicada arriba.

Con esta adimensionalización así definida y, adoptando como estado de referencia a la corriente no perturbada, las expresiones para la viscosidad y conductividad térmica adimensionales resultan:

$$
\mu=\frac{M_{\infty}}{R_{e_{\infty}}} \frac{S+u_{\infty}}{S+u}\left(\frac{u}{u_{\infty}}\right)^{\frac{3}{2}} ; \quad K=\frac{M_{\infty}}{R_{e_{\infty}}} \frac{\gamma}{P r_{\infty}} \frac{S_{k}+u_{\infty}}{S_{k}+u}\left(\frac{u}{u_{\infty}}\right)^{\frac{3}{2}}
$$

En donde:

$$
R_{e_{\infty}}=\frac{\rho_{\infty}^{*} V_{\infty}^{*} L_{r e f}^{*}}{\mu_{\infty}^{*}}
$$

es el número de Reynolds no perturbado, y: 


$$
\begin{gathered}
M=\frac{\sqrt{v_{i}{ }^{*} v_{i}{ }^{*}}}{c^{*}}=\frac{\sqrt{v_{i} v_{i}}}{c} ; \quad M_{\infty}=\frac{V_{\infty}^{*}}{c_{\infty}^{*}}=\frac{V_{\infty}}{c_{\infty}} ; \quad c^{*}=\sqrt{\gamma R^{*} T^{*}} ; \\
c=\frac{c^{*}}{c_{\infty}^{*}}=\sqrt{\gamma(\gamma-1) u} ; \quad \gamma=\frac{c_{p}{ }^{*}}{c_{v}{ }^{*}}=1,4 ; \quad R^{*}=287 \frac{\mathrm{J}}{\mathrm{kgK}}
\end{gathered}
$$

son el número de Mach local, el número de Mach no perturbado, la velocidad del sonido local, la velocidad del sonido local adimensional, la relación de calores específicos y la constante particular del aire de la ecuación de estado. El número de Prandtl se adopta constante, resultando

$$
\operatorname{Pr}_{\infty}=\frac{c_{p}^{*} \mu_{\infty}^{*}}{K_{\infty}^{*}}=0,72
$$

Finalmente, los coeficientes $S$ y $S_{K}$ deben expresarse en forma adimensional según las siguientes expresiones:

$$
S=\frac{c_{v}{ }^{*} S^{*}}{c_{\infty}^{* 2}} ; S_{K}=\frac{c_{v}{ }^{*} S_{K}^{*}}{c_{\infty}^{* 2}}
$$

\subsection{ECUACIONES FANS (FAVRE AVERAGED NAVIER-STOKES).}

\subsubsection{Promediado de las ecuaciones de Navier-Stokes:}

Cuando se reemplazan las tensiones y las componentes del vector de flujo de calor en las ecuaciones de conservación por las que resultan de las ecuaciones constitutivas para un fluido newtoniano, se obtienen las conocidas ecuaciones de Navier-Stokes. Estas ecuaciones podrían utilizarse en forma directa para hacer las simulaciones de los diferentes escurrimientos (DNS); sin embargo, deberían utilizarse mallas extremadamente finas dado que las mismas deben ser adecuadas para capturar las escalas más pequeñas de la turbulencia.

Como se indicó en el capítulo 1, para evitar esto, otra forma de tratar la turbulencia es por medio de la resolución de ecuaciones de Navier-Stokes promediadas (RANS, Reynolds Averaged Navier-Stokes, o FANS, Favre Averaged Navier-Stokes). En este enfoque se transforman las ecuaciones de Navier-Stokes en un modelo matemático similar, pero con variables promediadas en el tiempo, de manera que las ecuaciones resultantes sólo ofrecen una solución para las variables promediadas ${ }^{[42]}$.

Osbourne Reynolds [43] propuso hacer una descomposición de las variables, separándolas en su media temporal más sus fluctuaciones temporales turbulentas, es decir, si $f$ es una variable cualquiera: 


$$
f=\bar{f}+f^{\prime}
$$

en donde $\bar{f}$ es la media temporal definida mediante el siguiente filtro:

$$
\bar{f}=\frac{1}{2 T} \int_{-T}^{T} f d t
$$

siendo $T$ un período de tiempo representativo, y las fluctuaciones turbulentas quedan definidas por la siguiente expresión:

$$
f^{\prime}=f-\bar{f}
$$

Se desprende, entonces, que:

$$
\overline{f^{\prime}}=\frac{1}{2 T} \int_{-T}^{T}(f-\bar{f}) d t=0 ; \quad \overline{\bar{f}}=\bar{f}
$$

Aplicando esta descomposición a las ecuaciones de Navier-Stokes se obtienen las ecuaciones RANS (Reynolds Averaged Navier-Stokes) que constituyen un sistema de ecuaciones útil para obtener las variables promediadas según se mostró arriba, es decir, $\overline{v_{l}}, \bar{p}$, entre otras.

Sin embargo, el procedimiento anterior es particularmente práctico para el caso de escurrimientos incompresibles, donde la densidad es considerada constante y donde la ecuación de energía generalmente se considera desacoplada, por lo cual no se consideran fluctuaciones de densidad y temperatura. Pero en casos compresibles, donde las fluctuaciones de densidad y temperatura (energía interna adimensional, con la adimensionalización utilizada en este trabajo) deben ser consideradas, las ecuaciones RANS obtenidas con el filtrado de Reynolds mostrado más arriba conducen a un sistema de ecuaciones bastante complicado.

En casos donde los efectos de compresibilidad son importantes suele utilizarse el filtro de Favre ${ }^{[44]}$, definido por las siguientes expresiones:

$$
\begin{gathered}
f=\tilde{f}+f^{\prime \prime} \\
\tilde{f}=\frac{\int_{-T}^{T} \rho f d t}{\int_{-T}^{T} \rho d t}=\frac{\overline{\rho f}}{\bar{\rho}}
\end{gathered}
$$

en donde la barra sigue indicando el filtro de Reynolds y la tilde indica el filtro de Favre.

Considerando el filtro de Favre, algunas relaciones auxiliares importantes son las siguientes: 


$$
\overline{\rho f^{\prime \prime}}=0 ; \quad \overline{\rho \tilde{f}}=\bar{\rho} \tilde{f}=\overline{\rho f} ; \quad \overline{f^{\prime \prime}} \neq 0
$$

Y, considerando que el filtro de Favre conmuta con la derivada, entonces, aplicando este filtro a las ecuaciones de Navier-Stokes ya adimensionalizadas se obtienen las siguientes expresiones ${ }^{[45]}$ :

Ecuación de conservación de masa:

$$
\frac{\partial \bar{\rho}}{\partial t}+\frac{\partial \bar{\rho} \widetilde{v}_{l}}{\partial x_{i}}=0
$$

Ecuaciones de conservación de la cantidad de movimiento:

$$
\frac{\partial\left(\bar{\rho} \widetilde{v_{J}}\right)}{\partial t}+\frac{\partial\left(\bar{\rho} \widetilde{v_{j}} \widetilde{v_{l}}\right)}{\partial x_{i}}+\frac{\partial \bar{p}}{\partial x_{j}}-\frac{\partial \widetilde{\tau_{l j}}}{\partial x_{i}}-\frac{\partial \tau_{t i j}}{\partial x_{i}}=0
$$

Ecuación de conservación de energía:

$$
\begin{aligned}
\frac{\partial(\bar{\rho} \tilde{e})}{\partial t}+ & \frac{\partial(\bar{\rho} \tilde{e}+\bar{p}) \widetilde{v}_{l}}{\partial x_{i}}-\frac{\partial\left(\widetilde{v_{J}} \widetilde{\tau_{l \jmath}}\right)}{\partial x_{i}}+\frac{\partial\left(\widetilde{v_{j}} \tau_{t i j}\right)}{\partial x_{i}}+\frac{\partial \widetilde{q_{l}}}{\partial x_{i}}+\frac{\partial q_{t i}}{\partial x_{i}}-\frac{\partial\left(\overline{v_{\jmath}^{\prime \prime} \tau_{l \jmath}}\right)}{\partial x_{i}} \\
& +\frac{1}{2} \frac{\partial\left(\overline{\rho v_{j}^{\prime \prime} v_{j}^{\prime \prime} v_{l}^{\prime \prime}}\right)}{\partial x_{i}}=0
\end{aligned}
$$

Siendo:

$$
\begin{aligned}
& \tau_{t i j}=-\overline{\rho v_{l}^{\prime \prime} v_{\jmath}^{\prime \prime}} \\
& \widetilde{\tau_{l \jmath}}=2 \tilde{\mu}\left[\widetilde{S_{l j}}-\frac{1}{3} \frac{\partial \widetilde{v_{k}}}{\partial x_{k}} \delta_{i j}\right] ; \quad \widetilde{S_{l \jmath}}=\frac{1}{2}\left[\frac{\partial \widetilde{v_{l}}}{\partial x_{j}}+\frac{\partial \widetilde{v_{j}}}{\partial x_{i}}\right] \\
& q_{t i}=\overline{\rho v_{l}^{\prime \prime} u^{\prime \prime}} \\
& \widetilde{q_{l}}=-\widetilde{K} \frac{\partial \tilde{u}}{\partial x_{i}} \\
& \tilde{e}=\tilde{u}+\frac{1}{2} \widetilde{v}_{l} \widetilde{v}_{l}+k_{t} ; \quad k_{t}=\frac{1}{2} \overline{v_{l}^{\prime \prime} v_{l}^{\prime \prime}} \\
& \tilde{\mu}=\frac{M_{\infty}}{R_{e_{\infty}}} \frac{S+u_{\infty}}{S+\tilde{u}}\left(\frac{\tilde{u}}{u_{\infty}}\right)^{\frac{3}{2}} ; \quad \widetilde{K}=\frac{M_{\infty}}{R_{e_{\infty}}} \frac{\gamma}{P r_{\infty}} \frac{S_{k}+u_{\infty}}{S_{k}+\tilde{u}}\left(\frac{\tilde{u}}{u_{\infty}}\right)^{\frac{3}{2}} \\
& \bar{p}=(\gamma-1) \bar{\rho} \tilde{u}
\end{aligned}
$$

Así definido, este sistema de ecuaciones puede solucionarse para obtener las variables $\widetilde{v_{l}} ; \bar{\rho} ; \tilde{u}$ y $\bar{p}$. Constituye, por lo tanto, un nuevo sistema de ecuaciones que no permite obtener la solución del problema original sino la solución para las variables filtradas. Además, 
para poder cerrar este sistema de ecuaciones y resolverlo existen cinco correlaciones que necesitan ser modeladas, a saber:

$\checkmark \quad \tau_{t i j}=-\overline{\rho v_{l}^{\prime \prime} v_{J}^{\prime \prime}}$ son las tensiones turbulentas o tensiones de Reynolds (Favre),

$\checkmark q_{t i}=\overline{\rho v_{l}^{\prime \prime} u^{\prime \prime}}$ es el flujo de calor turbulento,

$\checkmark-\overline{v_{\jmath}^{\prime \prime} \tau_{\iota \jmath}}$ son las componentes de la disipación de energía turbulenta,

$\checkmark \overline{\rho v_{J}^{\prime \prime} v_{J}^{\prime \prime} v_{l}^{\prime \prime}}$ es la difusión turbulenta,

$\checkmark k_{t}=\frac{1}{2} \overline{v_{l}^{\prime \prime} v_{l}^{\prime \prime}}$ es la energía cinética específica turbulenta adimensional.

Estos términos representan la "acción" que las fluctuaciones ejercen sobre la solución del problema entendida en términos de las variables filtradas $\mathrm{y}$, como puede inferirse de sus expresiones, son desconocidos si no se conocen las fluctuaciones turbulentas.

\subsubsection{Aproximación de Boussinesq:}

Como fue indicado más arriba, para cerrar el sistema de ecuaciones deben modelarse los términos que involucran fluctuaciones turbulentas. Esto significa proponer expresiones y ecuaciones que permitan estimar su valor en función de variables del problema sin utilizar las fluctuaciones turbulentas, dado que estas permanecerán desconocidas.

Esto condujo a la aparición de diferentes modelos de turbulencia, algunos de los cuales son más apropiados para determinados tipos de problemas, siendo que ninguno es universalmente válido $\mathrm{y}$, por lo tanto, preponderante sobre los otros.

Los modelos de turbulencia pueden ser de primer orden o segundo orden según el tratamiento que se les dé a las tensiones turbulentas $-\overline{\rho v_{l}^{\prime \prime} v_{J}^{\prime \prime}}\left(\mathrm{o}-\overline{\rho v_{l}^{\prime} v_{J}^{\prime}}\right.$ en las RANS).

Los denominados "modelos de primer orden", o también "modelos de viscosidad turbulenta" (EVM, Eddy Viscosity Models), utilizan la forma de las relaciones constitutivas para modelar a las tensiones turbulentas $\tau_{t i j}$. Este enfoque que propone la utilización de la forma de la relación constitutiva se conoce como aproximación de Boussinesq ${ }^{[46]}$. Estos modelos de primer orden, o modelos EVM, pueden ser lineales o no lineales.

Los denominados "modelos de segundo orden" resuelven las tensiones turbulentas $\tau_{t i j}$ utilizando ecuaciones de transporte o sistemas de ecuaciones algebraicas directamente, sin imponer una forma predefinida como en el caso de la aproximación de Boussinesq. 
En la siguiente figura pueden verse algunos de los modelos existentes y su categorización (LRR: Launder, Reece \& Rodi; GL: Gibson \& Launder; AMGS: Abid, Morrison, Gatski \& Speziale):

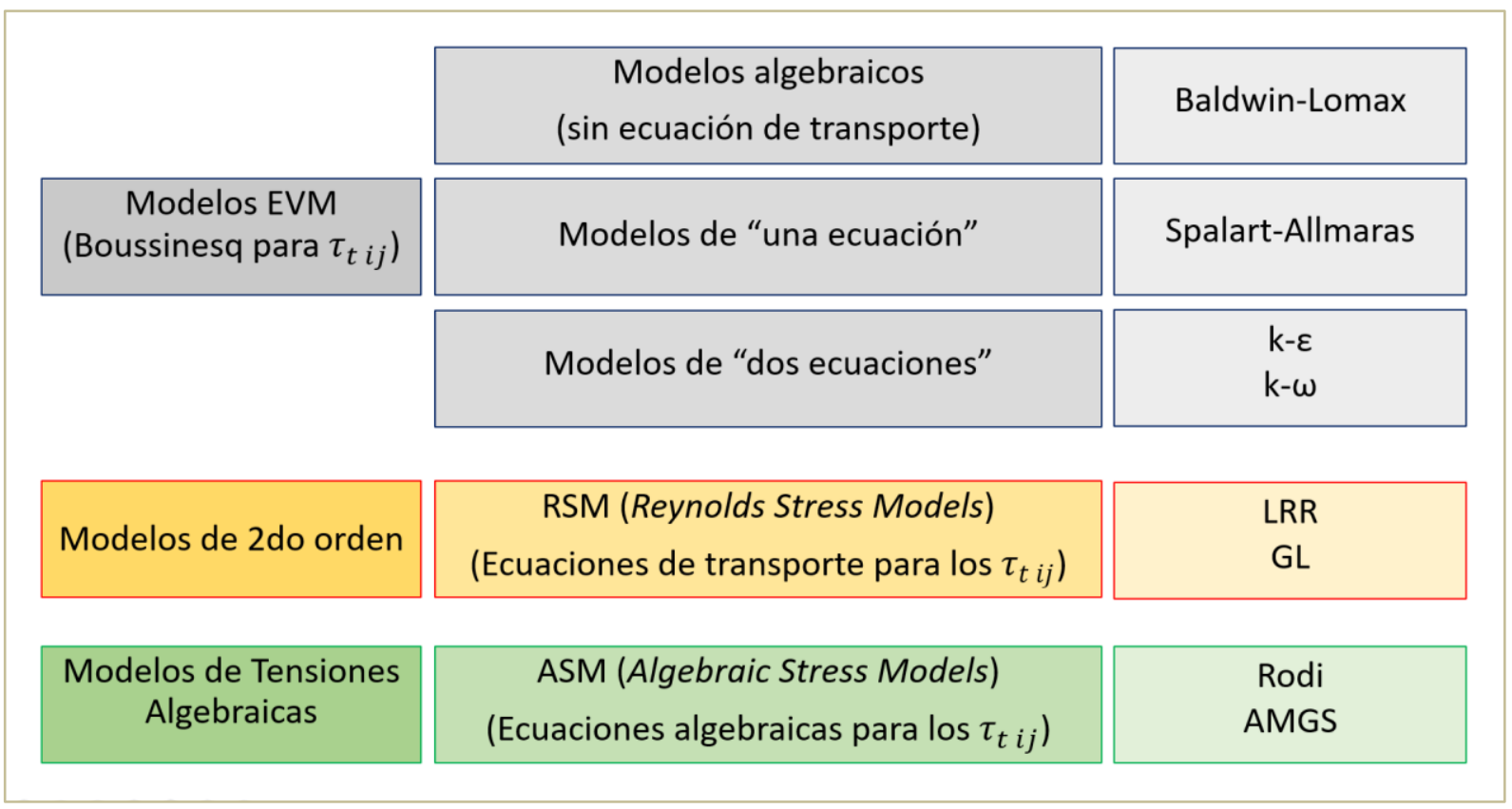

Figura 2.1: Algunos modelos de turbulencia y su clasificación.

En este trabajo se utiliza la aproximación de Boussinesq con el modelo de SpalartAllmaras que da buenos resultados en problemas de aerodinámica externa. Para las ecuaciones FANS definidas en las expresiones (2.21) a (2.30), la aproximación de Boussinesq adopta la forma siguiente:

$$
\tau_{t i j}=2 \mu_{t}\left(\widetilde{S_{l j}}-\frac{1}{3} \frac{\partial \widetilde{v_{k}}}{\partial x_{k}} \delta_{i j}\right)-\frac{2}{3} \bar{\rho} k_{t} \delta_{i j} ; \quad \widetilde{S_{l j}}=\frac{1}{2}\left[\frac{\partial \widetilde{v_{l}}}{\partial x_{j}}+\frac{\partial \widetilde{v_{j}}}{\partial x_{i}}\right]
$$

donde $\mu_{t}$ es la viscosidad turbulenta (eddy viscosity), $k_{t}$ es la energía cinética turbulenta y el subíndice $k$ corre de 1 a 3 . Ambas cantidades deberán ser obtenidas aplicando alguno de los modelos de turbulencia.

La aproximación de Boussinesq se puede generalizar para modelar al flujo de calor turbulento de la siguiente forma:

$$
q_{t i}=-K_{t} \frac{\partial \tilde{u}}{\partial x_{i}} ; \quad K_{t}=\mu_{t} \frac{\gamma}{P r_{t}}
$$

donde $K_{t}$ es la conductividad térmica turbulenta y $P r_{t}$ es el número de Prandtl turbulento el cual suele adoptarse como constante y para aire es igual a 0,9 para capas límite (wall bounded flow) y 0,5 para estelas de corte (shear layers). 
Los términos asociados a la disipación turbulenta y a la difusión turbulenta suelen modelarse en forma conjunta, como se muestra en la siguiente expresión:

$$
-\overline{v_{\jmath}^{\prime \prime} \tau_{l \jmath}}+\frac{1}{2} \overline{\rho v_{\jmath}^{\prime \prime} v_{\jmath}^{\prime \prime} v_{l}^{\prime \prime}} \cong-\left(\tilde{\mu}+\frac{\mu_{t}}{\sigma_{k}}\right) \frac{\partial k_{t}}{\partial x_{i}}
$$

en donde $\sigma_{k}$ es un coeficiente cuyo valor depende del modelo de turbulencia adoptado y está asociado a la forma de calcular el valor de $k_{t}$ de dicho modelo.

Por último, es importante recordar que todas las cantidades expresadas en este apartado 2.5 ya se encuentran adimensionalizadas según el procedimiento indicado en la sección 2.4. Como se indicó, previamente, todas las magnitudes que se utilizan a partir de ahora son adimensionales, a no ser que se especifique puntualmente lo contrario.

\subsubsection{Ecuaciones FANS con aproximación de Boussinesq:}

Finalmente, luego de adimensionalizar las ecuaciones de Navier-Stokes, aplicar el filtro de Favre y utilizar la aproximación de Boussinesq, resulta el siguiente sistema de ecuaciones que debe ser cerrado utilizando algún modelo para estimar los valores de $\mu_{t} \mathrm{y} k_{t}$ :

Ecuación de conservación de masa:

$$
\frac{\partial \rho}{\partial t}+\frac{\partial \rho v_{i}}{\partial x_{i}}=0
$$

Ecuaciones de conservación de la cantidad de movimiento:

$$
\frac{\partial\left(\rho v_{j}\right)}{\partial t}+\frac{\partial\left(\rho v_{j} v_{i}\right)}{\partial x_{i}}+\frac{\partial p}{\partial x_{i}}-\frac{\partial \tau_{i j}}{\partial x_{i}}=0
$$

Ecuación de conservación de energía:

$$
\frac{\partial(\rho e)}{\partial t}+\frac{\partial(\rho e+p) v_{i}}{\partial x_{i}}-\frac{\partial\left(v_{j} \tau_{i j}\right)}{\partial x_{i}}+\frac{\partial q_{i}}{\partial x_{i}}-\frac{\partial \psi_{i}}{\partial x_{i}}=0
$$

todas ellas son válidas en el dominio $\Omega$, sin términos fuente, y en todos los casos $i, j=1,2,3$.

Las relaciones constitutivas, considerando la viscosidad turbulenta y la conductividad térmica turbulenta, quedan definidas por las siguientes expresiones:

$$
\begin{gathered}
\tau_{i j}=\eta\left[\frac{\partial v_{i}}{\partial x_{j}}+\frac{\partial v_{j}}{\partial x_{i}}\right]+\lambda \frac{\partial v_{k}}{\partial x_{k}} \delta_{i j}-\frac{2}{3} \rho k_{t} \delta_{i j} ; \eta=\mu+\mu_{t} ; \lambda=-\frac{2}{3}\left(\mu+\mu_{t}\right) \\
q_{i}=-\kappa \frac{\partial u}{\partial x_{i}} ; \kappa=K+K_{t}
\end{gathered}
$$


siendo $\psi_{i}$ es el término difusivo-disipativo asociado a la energía cinética turbulenta $k_{t}$ en la ecuación de energía que viene dado por:

$$
\psi_{i}=\left(\mu+\frac{\mu_{t}}{\sigma_{k}}\right) \frac{\partial k_{t}}{\partial x_{i}}
$$

Aquí se sobreentiende que las variables son las variables filtradas según se indicó en la sección 2.5.1, por lo cual de aquí en más ya no se utilizarán las barras y las tildes para indicar esta operación, la cual se da por sobreentendida, y se deja libre esta particular notación para otros usos.

\subsection{MODELO DE TURBULENCIA DE SPALART-ALLMARAS}

El modelo de turbulencia de Spalart-Allmaras es un modelo de una ecuación, porque utiliza una ecuación de transporte para determinar una variable $\tilde{v}$ asociada a la viscosidad turbulenta $\mu_{t}$ mientras que no se calcula la energía cinética turbulenta, por lo cual automáticamente se desprecian todos los términos que la contienen en el sistema de ecuaciones. A diferencia de otros modelos de dos ecuaciones en los que generalmente se utiliza una ecuación de transporte para la energía cinética turbulenta, en este modelo, como ya se indicó, esta correlación no se modela ${ }^{[33]}$.

Se define una variable $\tilde{v}$ (nu tilde) cuyas dimensiones son las de la viscosidad cinemática y se utiliza la siguiente ecuación de transporte para determinarla:

$$
\frac{\partial(\rho \tilde{v})}{\partial t}+\frac{\partial\left(\rho \tilde{v} v_{i}\right)}{\partial x_{i}}-\frac{\partial \varphi_{i}}{\partial x_{i}}+P+D+D_{T}=0
$$

$\checkmark \quad \tilde{v}$ es la variable de la ecuación de transporte del modelo de Spalart-Allmaras.

$\checkmark \varphi_{i}$ es el término difusivo de la ecuación de transporte de Spalart-Allmaras.

$\checkmark \quad P$ es el término de producción y $D$ el de destrucción de la ecuación de transporte de Spalart-Allmaras.

$\checkmark \quad D_{T}$ contiene términos adicionales de la ecuación de transporte de Spalart-Allmaras.

El modelo de turbulencia de Spalart-Allmaras no contempla el cálculo de la energía cinética turbulenta por lo cual todos los términos que la contienen se desprecian, incluyendo $\psi_{i}$ en (2.36) y el tercer término en (2.37).

Los términos $\varphi_{i}$ en la ecuación de transporte de Spalart-Allmaras vienen dados por la siguiente expresión: 


$$
\varphi_{i}=\frac{1}{\sigma}(\mu+\rho \tilde{v}) \frac{\partial \tilde{v}}{\partial x_{i}}
$$

donde $\sigma y_{c b_{2}}$ son constantes del modelo de turbulencia de Spalart-Allmaras. La ecuación de transporte viene acompañada por los términos adicionales $D_{T}$ que se expresan a continuación:

$$
D_{T}=-\frac{1}{\sigma} \rho c b_{2} \frac{\partial \tilde{v}}{\partial x_{i}} \frac{\partial \tilde{v}}{\partial x_{i}}+\frac{1}{\sigma}(v+\tilde{v}) \frac{\partial \rho}{\partial x_{i}} \frac{\partial \tilde{v}}{\partial x_{i}}
$$

Finalmente, los términos de producción y destrucción en la ecuación de transporte de Spalart-Allmaras se expresan de la siguiente forma:

$$
P=-c b_{1} S \rho \tilde{v} ; \quad D=c w_{1} f_{w} \rho\left(\frac{\tilde{v}}{d}\right)^{2}
$$

donde el primer término representa la producción y el segundo la destrucción, $c b_{1}, S, c w_{1}$ y $f_{w}$ son constantes y variables del modelo de turbulencia de Spalart-Allmaras y $d$ es la menor distancia del punto (o nodo) considerado al contorno sólido más cercano. La ecuación de transporte utilizada aquí está expresada en su forma conservativa, tal como puede encontrársela en el trabajo de S. R. Allmaras, F. T. Johnson y P. R. Spalart (2012) ${ }^{[40]}$ pero sin considerar los términos de supresión laminar y "trip terms" incluidos en las expresiones originales del modelo.

Para cerrar el sistema de ecuaciones aún es necesario definir todos los parámetros asociados al modelo de turbulencia de Spalart-Allmaras, los cuales se muestran a continuación:

$$
\begin{gathered}
\sigma=\frac{2}{3} ; c b_{1}=0,1355 ; c b_{2}=0,622 \\
S=\Omega+f_{v 2} \frac{\tilde{v}}{\mathrm{~K}^{2} d^{2}} ; \mathrm{K}=0,41 \\
f_{v 2}=1-\frac{\chi}{1+\chi f_{v 1}} ; \quad \chi=\frac{\rho \tilde{v}}{\mu} ; \quad f_{v 1}=\frac{\chi^{3}}{\chi^{3}+c_{v 1}{ }^{3}} ; \quad c_{v 1}=7,1 \\
f_{w}=g\left(\frac{1+c_{w 3}^{6}}{g^{6}+c_{w 3}{ }^{6}}\right)^{\frac{1}{6}} ; \quad c_{w 3}=2,0 ; \quad g=r+c_{w 2}\left(r^{6}-r\right) ; \quad c_{w 2}=0,3 \\
r=\frac{\tilde{v}}{S \mathrm{~K}^{2} d^{2}} ; \quad c_{w 1}=\frac{c b_{1}}{\mathrm{~K}^{2}}+\frac{1+c b_{2}}{\sigma}
\end{gathered}
$$

donde $\Omega$ es el módulo de la vorticidad dada por: 


$$
\mathbf{\Omega}=\operatorname{rot} \boldsymbol{V} ; \Omega=\sqrt{\mathbf{\Omega} \cdot \mathbf{\Omega}}
$$

La viscosidad turbulenta y la conductividad térmica turbulenta quedan definidas de la siguiente manera:

$$
\mu_{t}=\rho \tilde{v} f_{v 1} ; \quad K_{t}=\mu_{t} \frac{\gamma}{P r_{t}} ; \quad \gamma=\frac{c_{p}}{c_{v}}=1,4
$$

donde $P r_{t}$ es el número de Prandtl turbulento el cual se adopta $P r_{t}=0,9$ y $c_{v}$ y $c_{p}$ son los coeficientes de calor específico a volumen constante y a presión constante respectivamente.

\subsection{ECUACIONES FANS INCLUYENDO MODELO DE SPALART-ALLMARAS EN FORMA CONSERVATIVA COMPACTA.}

En una descripción Euleriana, el sistema de ecuaciones en derivadas parciales que gobierna la dinámica de gases compresibles, adimensionalizadas según se indicó en la sección 2.4, filtradas con un filtro de Favre, utilizando la aproximación de Boussinesq e incluyendo el modelo de Spalart-Allmaras, puede escribirse, en su forma conservativa, como sigue:

$$
\frac{\partial \boldsymbol{U}}{\partial t}+\frac{\partial \boldsymbol{F}_{\boldsymbol{i}}}{\partial x_{i}}+\frac{\partial \boldsymbol{G}_{i}}{\partial x_{i}}+\boldsymbol{Q}=\mathbf{0}
$$

Siendo:

$$
\begin{gathered}
\boldsymbol{U}=\left\{\begin{array}{c}
\rho \\
\rho v_{1} \\
\rho v_{2} \\
\rho v_{3} \\
\rho e \\
\rho \tilde{v}
\end{array}\right\} ; \boldsymbol{F}_{i=}\left\{\begin{array}{c}
\rho v_{i} \\
\rho v_{1} v_{i}+p \delta_{i 1} \\
\rho v_{2} v_{i}+p \delta_{i 2} \\
\rho v_{3} v_{i}+p \delta_{i 3} \\
v_{i}(\rho e+p) \\
\rho \tilde{v} v_{i}
\end{array}\right\} ; \boldsymbol{G}_{i}=\left\{\begin{array}{c}
0 \\
-\tau_{i 1} \\
-\tau_{i 2} \\
-\tau_{i 3} \\
-\tau_{i j} v_{j}+q_{i}-\psi_{i} \\
-\varphi_{i}
\end{array}\right\} ; \\
\boldsymbol{Q}=\left\{\begin{array}{c}
0 \\
0 \\
0 \\
0 \\
0 \\
P+D+D_{T}
\end{array}\right\}
\end{gathered}
$$

Las tensiones $\tau_{i j}$, las componentes del flujo de calor por conducción $q_{i}$, y los términos $\psi_{i}, \varphi_{i}, P, D$ y $D_{T}$ se calculan como se indicó en las expresiones (2.37) a (2.39) y (2.41) a (2.43) respectivamente. El término $\psi_{i}$ se incluyó aquí para mostrar las ecuaciones en su forma más completa, pero éste se desprecia ya que la energía cinética turbulenta no se considera.

Como se observó anteriormente, deben considerarse, además, la ecuación de estado (2.30), la relación entre las energías específicas involucradas (2.28) y una ley de variación de la 
viscosidad y el coeficiente de conductividad térmica con la temperatura (2.29), las cuales se repiten aquí en aras a la completitud:

$$
\begin{gathered}
p=(\gamma-1) \rho u \\
e=u+\frac{1}{2} v_{i} v_{i}+k_{t}
\end{gathered}
$$

aunque aquí $k_{t}$ se desprecia. Además:

$$
\mu=\frac{M_{\infty}}{R_{e_{\infty}}} \frac{S+u_{\infty}}{S+u}\left(\frac{u}{u_{\infty}}\right)^{\frac{3}{2}} ; \quad K=\frac{M_{\infty}}{R_{e_{\infty}}} \frac{\gamma}{\operatorname{Pr}_{\infty}} \frac{S_{k}+u_{\infty}}{S_{k}+u}\left(\frac{u}{u_{\infty}}\right)^{\frac{3}{2}}
$$

Por último, deben aplicarse condiciones iniciales y de contorno a las ecuaciones (2.48) para tener el modelo matemático correctamente definido. Las condiciones forzadas de contorno (o condiciones de contorno de Dirichlet) vienen dadas por:

$$
\begin{array}{lll}
v_{i}=\bar{v}_{\iota} & \text { en } & \Gamma_{v} \\
\rho=\bar{\rho} & \text { en } & \Gamma_{\rho} \\
u=\bar{u} & \text { en } & \Gamma_{u} \\
\tilde{v}=\overline{\tilde{v}} & \text { en } & \Gamma_{\widetilde{v}}
\end{array}
$$

donde $\bar{v}_{l}, \bar{\rho}, \bar{u}$ y $\overline{\tilde{v}}$ son los valores prescriptos de las componentes de velocidad, la densidad (masa específica), la energía interna específica y la variable $\tilde{v}$, en las regiones de la superficie de contorno $\Gamma_{v}, \Gamma_{\rho}, \Gamma_{u} \mathrm{y} \Gamma_{\widetilde{v}}$ respectivamente.

Las condiciones naturales de contorno (o condiciones de contorno de Neumann) vienen definidas, a su vez, por las siguientes expresiones:

$$
\begin{gathered}
\sigma_{i j} n_{j}=\widehat{t_{l}} \text { en } \Gamma_{\sigma} \\
q_{i} n_{i}=\hat{q} \quad \text { en } \Gamma_{q}
\end{gathered}
$$

En las expresiones (2.53) y (2.54) se tiene:

$\checkmark \quad n_{i}$ son los cosenos de los ángulos formados por el vector normal a la superficie $\Gamma_{\sigma}$ o $\Gamma_{q}$ y los ejes de referencia globales $x_{i}$,

$\checkmark \widehat{t}_{l}$ son las tracciones actuando sobre $\Gamma_{\sigma}$,

$\checkmark \hat{q}$ es el flujo de calor normal actuando en $\Gamma_{q}$,

$\checkmark$ los efectos de la radiación y la convección en las superficies de contorno no han sido considerados. 
Para flujos no viscosos la ecuación de transporte de la variable $\tilde{v}$ y los términos difusivos $\boldsymbol{G}_{i}$ en las ecuaciones (2.48) deben omitirse. En este caso, sólo se prescribe la componente de velocidad normal al contorno sólido (nula) y sólo se tienen en cuenta las componentes normales de las cargas superficiales en el contorno del dominio. 


\section{FORMULACIONES DE ELEMENTOS FINITOS.}

\subsection{EL ESQUEMA DE TAYLOR-GALERKIN.}

\subsubsection{Avance en el tiempo: serie de Taylor.}

El esquema de Taylor-Galerkin (TG) fue introducido por Donea ${ }^{[15]}$ en 1984 y consiste en una expansión temporal en series de Taylor y posterior discretización en el espacio utilizando el método de Bubnov-Galerkin.

Para la discretización temporal, el vector $\boldsymbol{U}$ se puede expandir según una serie de Taylor como sigue, en donde se han omitido los términos de orden superior (Yoon et. al. ${ }^{[47]}$ ):

$$
\Delta \boldsymbol{U}^{n+1}=\Delta t\left(\frac{\partial \boldsymbol{U}}{\partial t}\right)^{n+s_{1}}+\frac{\Delta t}{2}\left(\frac{\partial^{2} \boldsymbol{U}}{\partial t^{2}}\right)^{n+s_{2}}+\cdots
$$

donde $\Delta t$ es el paso de tiempo, $n$ corresponde al tiempo $t$ (paso de tiempo anterior), $n+1$ corresponde al tiempo $t+\Delta t$ (paso de tiempo actual). Los índices $S_{1}$ y $S_{2}$ determinan si el esquema será implícito o explícito, dependiendo de los valores que adopten, y en la referencia [47] se los indica como implicitness parameters.

Expandiendo las derivadas en (3.1)

$$
\begin{array}{clrl}
\frac{\partial \boldsymbol{U}^{n+s_{1}}}{\partial t} & =\frac{\partial \boldsymbol{U}^{n}}{\partial t}+s_{1} \frac{\partial \Delta \boldsymbol{U}^{n+1}}{\partial t} & 0 \leq s_{1} \leq 1 \\
{\frac{\partial^{2} \boldsymbol{U}^{n+s_{2}}}{\partial t^{2}}}^{=}=\frac{\partial^{2} \boldsymbol{U}^{n}}{\partial t^{2}}+s_{2} \frac{\partial^{2} \Delta \boldsymbol{U}^{n+1}}{\partial t^{2}} & 0 \leq s_{2} \leq 1
\end{array}
$$

Adoptando los valores $S_{1}=\frac{1}{2}$ y $S_{2}=\frac{1}{2}$ y reemplazando en (3.1), resulta:

$$
\Delta \boldsymbol{U}^{n+1}=\Delta t\left(\frac{\partial \boldsymbol{U}^{n}}{\partial t}+\frac{1}{2} \frac{\partial \Delta \boldsymbol{U}^{n+1}}{\partial t}\right)+\frac{\Delta t^{2}}{2}\left(\frac{\partial^{2} \boldsymbol{U}^{n}}{\partial t^{2}}+\frac{1}{2} \frac{\partial^{2} \Delta \boldsymbol{U}^{n+1}}{\partial t^{2}}\right)
$$

Se busca, luego, reemplazar las derivadas temporales de $\boldsymbol{U}^{n}$ y $\Delta \boldsymbol{U}^{n+1}$ en (3.4). De (2.47) se tiene: 


$$
\frac{\partial \boldsymbol{U}^{n}}{\partial t}=-{\frac{\partial \boldsymbol{F}_{i}}{\partial x_{i}}}^{n}-\frac{\partial \boldsymbol{G}_{i}{ }^{n}}{\partial x_{i}}-\boldsymbol{Q}^{n}
$$

Derivado la expresión (3.5), se obtiene:

$$
\begin{gathered}
\frac{\partial^{2} \boldsymbol{U}^{n}}{\partial t^{2}}=\frac{\partial}{\partial t}\left(-\frac{\partial \boldsymbol{F}_{i}{ }^{n}}{\partial x_{i}}-\frac{\partial \boldsymbol{G}_{i}{ }^{n}}{\partial x_{i}}-\boldsymbol{Q}^{n}\right) \\
\frac{\partial^{2} \boldsymbol{U}^{n}}{\partial t^{2}}=\frac{\partial}{\partial x_{i}}\left(-\frac{\partial \boldsymbol{F}_{i}{ }^{n}}{\partial t}-\frac{\partial \boldsymbol{G}_{i}{ }^{n}}{\partial t}\right)-\frac{\partial \boldsymbol{Q}^{n}}{\partial t}
\end{gathered}
$$

Usando la regla de cadena, se tiene:

$$
{\frac{\partial^{2} \boldsymbol{U}^{n}}{\partial t^{2}}}^{n}=\frac{\partial}{\partial x_{i}}\left(-\frac{\partial \boldsymbol{F}_{i}{ }^{n}}{\partial \boldsymbol{U}} \frac{\partial \boldsymbol{U}^{n}}{\partial t}-\frac{\partial \boldsymbol{G}_{i}{ }^{n}}{\partial \boldsymbol{U}} \frac{\partial \boldsymbol{U}^{n}}{\partial t}\right)-\frac{\partial \boldsymbol{Q}^{n}}{\partial t}
$$

Denominando:

$$
\frac{\partial \boldsymbol{F}_{i}{ }^{n}}{\partial \boldsymbol{U}}=\boldsymbol{A}_{i}^{n} ; \quad{\frac{\partial \boldsymbol{G}_{i}{ }^{n}}{\partial \boldsymbol{U}}}^{=}=\boldsymbol{B}_{i}^{n}
$$

donde $\boldsymbol{A}_{i}^{n}$ y $\boldsymbol{B}_{i}^{n}$ son matrices de 6 x6. Observando que $\boldsymbol{B}_{i}^{n}$ involucra derivadas de orden superior a las que aparecen en $\boldsymbol{A}_{i}^{n}$, se desconsidera la matriz $\boldsymbol{B}_{i}^{n}$, o sea que se adopta lo siguiente:

$$
\boldsymbol{B}_{i}^{n}=\mathbf{0}_{i}^{n}
$$

donde $\mathbf{0}_{i}^{n}$ es una matriz con todos sus elementos nulos. Aplicando diferencias finitas avanzadas al término fuente se tiene que:

$$
\frac{\partial \boldsymbol{Q}^{n}}{\partial t}=\frac{\Delta \boldsymbol{Q}^{n+1}}{\Delta t}=\frac{\boldsymbol{Q}^{n+1}-\boldsymbol{Q}^{n}}{\Delta t}
$$

resultando, entonces, lo siguiente:

$$
\frac{\partial^{2} \boldsymbol{U}^{n}}{\partial t^{2}}=\frac{\partial}{\partial x_{i}}\left(-\boldsymbol{A}_{i}{ }^{n} \frac{\partial \boldsymbol{U}^{n}}{\partial t}\right)-\frac{\Delta \boldsymbol{Q}^{n+1}}{\Delta t}
$$

Las expresiones (3.5) y (3.12) también son válidas para los incrementos, es decir:

$$
{\frac{\partial \Delta \boldsymbol{U}^{n+1}}{\partial t}}^{n+}=-\frac{\partial \Delta \boldsymbol{F}_{i}{ }^{n+1}}{\partial x_{i}}-\frac{\partial \Delta \boldsymbol{G}_{i}^{n+1}}{\partial x_{i}}-\Delta \boldsymbol{Q}^{n+1}
$$

y, despreciando el incremento de $\Delta \boldsymbol{Q}$, se obtiene:

$$
{\frac{\partial^{2} \Delta \boldsymbol{U}^{n+1}}{\partial t^{2}}}^{n}=\frac{\partial}{\partial x_{i}}\left(-\boldsymbol{A}_{i}{ }^{n} \frac{\partial \Delta \boldsymbol{U}^{n+1}}{\partial t}\right)
$$


En (3.4) deben reemplazarse las derivadas segundas temporales por las expresiones (3.12) y (3.14). Luego, sobre la expresión obtenida, se reemplazan las derivadas temporales usando las expresiones (3.5) y (3.13). Haciendo caso omiso de los términos con derivadas segundas de los términos difusivos con respecto a la posición y reagrupando términos, se obtiene la siguiente ecuación para los incrementos de las variables:

$$
\begin{aligned}
\Delta \boldsymbol{U}^{n+1}= & \Delta t\left[-\frac{\partial \boldsymbol{F}_{i}^{n}}{\partial x_{i}}-\frac{\partial \boldsymbol{G}_{i}^{n}}{\partial x_{i}}-\boldsymbol{Q}^{n}+\frac{\Delta t}{2} \frac{\partial}{\partial x_{k}}\left(\boldsymbol{A}_{k}{ }^{n} \frac{\partial \boldsymbol{F}_{i}{ }^{n}}{\partial x_{i}}+\boldsymbol{Q}^{n}\right)\right] \\
& +\frac{\Delta t}{2}\left[-\frac{\partial \Delta \boldsymbol{F}_{i}{ }^{n+1}}{\partial x_{i}}-\frac{\partial \Delta \boldsymbol{G}_{i}{ }^{n+1}}{\partial x_{i}}-\Delta \boldsymbol{Q}^{n+1}+\frac{\Delta t}{2} \frac{\partial}{\partial x_{k}}\left(\boldsymbol{A}_{k}{ }^{n} \frac{\partial \Delta \boldsymbol{F}_{i}{ }^{n+1}}{\partial x_{i}}\right)\right]
\end{aligned}
$$

donde $\Delta t$ es el paso de tiempo, $n$ corresponde al tiempo $t, n+1$ corresponde al tiempo $t+\Delta t, \mathrm{y}$ $\Delta \boldsymbol{F}_{i}{ }^{n+1}=\boldsymbol{F}_{i}{ }^{n+1}-\boldsymbol{F}_{i}{ }^{n} ; \quad \Delta \boldsymbol{G}_{i}{ }^{n+1}=\boldsymbol{G}_{i}{ }^{n+1}-\boldsymbol{G}_{i}{ }^{n} ; \quad \Delta \boldsymbol{Q}^{n+1}=\boldsymbol{Q}^{n+1}-\boldsymbol{Q}^{n}$.

El cuarto término en el primer corchete de la expresión (3.15), que está multiplicado por $\Delta t / 2$, es un término estabilizador de segundo orden que actúa agregando cierto amortiguamiento numérico. Este término permite contrarrestar las oscilaciones numéricas que aparecen en problemas fuertemente convectivos, cuando se aplica el método de BubnovGalerkin para la discretización en el espacio. Dado que este problema es característico de los términos convectivos, la estabilización sobre el término difusivo y el término fuente no son estrictamente necesarias, lo cual hizo factible eliminar al término $\boldsymbol{G}_{i}$ y también haría factible eliminar al término $\boldsymbol{Q}^{n}$ dentro del término estabilizador.

Los términos que están en el segundo corchete en la expresión (3.15) son de segundo orden, y aparecen por haber adoptado los parámetros $s_{1}=1 / 2$ y $s_{2}=1 / 2$ con lo cual el esquema obtenido es implícito, ya que en dicha expresión las variables en el tiempo $n+1$ aparecen en ambos miembros de la ecuación. Esto puede observarse claramente en la expresión (3.4) notando que $\Delta \boldsymbol{U}^{n+1}$ aparece en ambos lados de la ecuación. Sin embargo, este esquema de avance en el tiempo podrá resolverse en forma explícita si, en lugar de pasar al lado izquierdo de la ecuación (3.15) todos los términos definidos en $n+1$, se utiliza un esquema iterativo para resolver los términos en el segundo corchete. Esto se verá con claridad en la próxima sección. La ecuación (3.15) se reescribe para indicar el paso iterativo como sigue:

$$
\begin{aligned}
\Delta \boldsymbol{U}_{I+1}^{n+1}= & \Delta t\left[-\frac{\partial \boldsymbol{F}_{i}{ }^{n}}{\partial x_{i}}-\frac{\partial \boldsymbol{G}_{i}{ }^{n}}{\partial x_{i}}-\boldsymbol{Q}^{n}+\frac{\Delta t}{2} \frac{\partial}{\partial x_{k}}\left(\boldsymbol{A}_{k}{ }^{n} \frac{\partial \boldsymbol{F}_{i}{ }^{n}}{\partial x_{i}}+\boldsymbol{Q}^{n}\right)\right] \\
& +\frac{\Delta t}{2}\left[-\frac{\partial \Delta \boldsymbol{F}_{i_{I}}^{n+1}}{\partial x_{i}}-\frac{\partial \Delta \boldsymbol{G}_{i_{I}}^{n+1}}{\partial x_{i}}-\Delta \boldsymbol{Q}_{I}^{n+1}+\frac{\Delta t}{2} \frac{\partial}{\partial x_{k}}\left(\boldsymbol{A}_{k}{ }^{n} \frac{\partial \Delta \boldsymbol{F}_{i_{I}}^{n+1}}{\partial x_{i}}\right)\right]
\end{aligned}
$$


donde se utiliza el subíndice " $P$ " para indicar iteración anterior e " $I+1$ ” iteración actual.

Los términos iterativos en (3.16) permiten acelerar la convergencia y, normalmente, tienen injerencia durante unos pocos pasos de tiempo en problemas donde se busca el estado estacionario a partir de un estado inicial a través de un transitorio ficticio. Más aún, según la experiencia adquirida por el autor de este trabajo, el término estabilizador iterativo no es necesario en (3.16), ya que resulta de tercer orden.

De esta manera, teniendo en cuenta todas las observaciones hechas anteriormente, puede plantearse una expresión simplificada sin mayor pérdida de exactitud, como se muestra a continuación:

$$
\begin{aligned}
\Delta \boldsymbol{U}_{I+1}^{n+1}= & \Delta t\left[-\frac{\partial \boldsymbol{F}_{i}^{n}}{\partial x_{i}}-\frac{\partial \boldsymbol{G}_{i}{ }^{n}}{\partial x_{i}}-\boldsymbol{Q}^{n}+\frac{\Delta t}{2} \frac{\partial}{\partial x_{k}}\left(\boldsymbol{A}_{k}{ }^{n} \frac{\partial \boldsymbol{F}_{i}{ }^{n}}{\partial x_{i}}\right)\right] \\
& +\frac{\Delta t}{2}\left[-\frac{\partial \Delta \boldsymbol{F}_{i_{I}}^{n+1}}{\partial x_{i}}-\frac{\partial \Delta \boldsymbol{G}_{i_{I}}^{n+1}}{\partial x_{i}}-\Delta \boldsymbol{Q}_{I}^{n+1}\right]
\end{aligned}
$$

Sin embargo, en este trabajo se continuará utilizando la expresión (3.16) ya que es más completa y a la hora de realizar la implementación en un código no resulta significativamente más onerosa.

Las matrices Jacobianas de Euler $\boldsymbol{A}_{k}$ (para $k=1,2,3$ ) son presentadas en el Anexo I en donde se desarrollan los términos $\boldsymbol{A}_{k}{ }^{n} \frac{\partial \boldsymbol{F}_{i}{ }^{n}}{\partial x_{i}}$ en detalle. Debe observarse que se trata de tres matrices $(k=1,2,3)$ de 6 x6 cuyos elementos son $A_{k_{m, l}}^{n}$ (para $\left.m, l=1,2, \ldots, 6\right)$.

Expandiendo la expresión (3.16) se obtiene, para cada una de las ecuaciones de conservación, lo siguiente:

Ecuación de conservación de masa:

$$
\begin{aligned}
\Delta \rho_{I+1}^{n+1} & =\Delta t\left[-\frac{\partial\left(\rho v_{i}\right)^{n}}{\partial x_{i}}+\frac{\Delta t}{2} \frac{\partial}{\partial x_{k}}\left(A_{k_{1, l}}^{n} \frac{\partial F_{i_{l}}^{n}}{\partial x_{i}}\right)\right] \\
& +\frac{\Delta t}{2}\left[-\frac{\partial\left(\Delta \rho v_{i}\right)_{I}^{n+1}}{\partial x_{i}}+\frac{\Delta t}{2} \frac{\partial}{\partial x_{k}}\left(A_{k_{1, l}}^{n} \frac{\partial \Delta\left(F_{i_{l}}\right)_{I}^{n+1}}{\partial x_{i}}\right)\right]
\end{aligned}
$$

$\operatorname{con} l=1,2, \ldots, 6$. 
Ecuación de conservación de cantidad de movimiento:

$$
\begin{aligned}
\Delta \rho v_{j_{I+1}}^{n+1}=\Delta t & -\frac{\partial\left(\rho v_{j} v_{i}+p \delta_{i j}\right)^{n}}{\partial x_{i}}+\frac{\partial \tau_{i j}{ }^{n}}{\partial x_{i}} \\
& \left.+\frac{\Delta t}{2} \frac{\partial}{\partial x_{k}}\left(A_{k_{m, l}}^{n} \frac{\partial F_{i_{l}}^{n}}{\partial x_{i}}\right)\right] \\
+\frac{\Delta t}{2} & {\left[-\frac{\partial \Delta\left(\rho v_{j} v_{i}+p \delta_{i j}\right)_{I}^{n+1}}{\partial x_{i}}+\frac{\partial \Delta \tau_{i j_{I}}^{n+1}}{\partial x_{i}}\right.} \\
& \left.+\frac{\Delta t}{2} \frac{\partial}{\partial x_{k}}\left(A_{k_{m, l}^{n}}^{n} \frac{\partial \Delta\left(F_{i_{l}}\right)_{I}^{n+1}}{\partial x_{i}}\right)\right]
\end{aligned}
$$

$\operatorname{con} l=1,2, \ldots, 6$ y $m=j+1$.

Ecuación de conservación de energía:

$$
\begin{aligned}
\Delta \rho e_{I+1}^{n+1}=\Delta t & -\frac{\partial\left[(\rho e+p) v_{i}\right]^{n}}{\partial x_{i}}+\frac{\partial\left(\tau_{i j} v_{j}\right)^{n}}{\partial x_{i}}+\frac{\partial \psi_{i}^{n}}{\partial x_{i}}-\frac{\partial q_{i}^{n}}{\partial x_{i}} \\
& \left.+\frac{\Delta t}{2} \frac{\partial}{\partial x_{k}}\left(A_{k_{5, l}}^{n} \frac{\partial F_{i_{l}}^{n}}{\partial x_{i}}\right)\right] \\
+ & \frac{\Delta t}{2}\left[-\frac{\partial \Delta\left[(\rho e+p) v_{i}\right]_{I}^{n+1}}{\partial x_{i}}+\frac{\partial \Delta\left(\tau_{i j} v_{j}\right)_{I}^{n+1}}{\partial x_{i}}+\frac{\partial \Delta \psi_{i_{I}}^{n+1}}{\partial x_{i}}\right. \\
& \left.-\frac{\partial \Delta q_{i_{I}}^{n+1}}{\partial x_{i}}+\frac{\Delta t}{2} \frac{\partial}{\partial x_{k}}\left(A_{k_{5, l}}^{n} \frac{\partial \Delta\left(F_{i_{l}}\right)_{I}^{n+1}}{\partial x_{i}}\right)\right]
\end{aligned}
$$

$\operatorname{con} l=1,2, \ldots, 6$.

Ecuación de transporte de $\tilde{v}$ del modelo de Spalart Allmaras (SA):

$$
\begin{aligned}
\Delta \rho \tilde{v}_{I+1}^{n+1}= & \Delta t\left[-\frac{\partial\left(\rho \tilde{v} v_{i}\right)^{n}}{\partial x_{i}}+\frac{\partial \varphi_{i}{ }^{n}}{\partial x_{i}}-P^{n}-D^{n}-D_{T}^{n}\right. \\
& \left.+\frac{\Delta t}{2} \frac{\partial}{\partial x_{k}}\left(A_{k_{6, l}}^{n} \frac{\partial F_{i_{l}}{ }^{n}}{\partial x_{i}}+P^{n}+D^{n}+D_{T}^{n}\right)\right] \\
& +\frac{\Delta t}{2}\left[-\frac{\partial \Delta\left(\rho \tilde{v} v_{i}\right)_{I}^{n+1}}{\partial x_{i}}+\frac{\partial \Delta \varphi_{i_{I}}^{n+1}}{\partial x_{i}}-\Delta P_{I}^{n+1}\right. \\
& \left.-\Delta D_{I}^{n+1}-\Delta D_{T_{I}}^{n+1}+\frac{\Delta t}{2} \frac{\partial}{\partial x_{k}}\left(A_{k_{6, l}}^{n} \frac{\partial \Delta\left(F_{i_{l}}\right)_{I}^{n+1}}{\partial x_{i}}\right)\right]
\end{aligned}
$$

$\operatorname{con} l=1,2, \ldots, 6$. 
En la expresión (3.20) los términos donde aparece $\psi_{i}$ no se consideran con el modelo de SA, mientras que en la expresión (3.21) los valores de $\varphi_{i}, P, D$ y $D_{T}$ surgen de las expresiones (2.41) a (2.43).

Se puede observar que, para los términos de estabilización, la matriz $\boldsymbol{A}_{k}^{n}$ provoca en cada ecuación de conservación un acoplamiento con todo el vector de flujo $\boldsymbol{F}_{i}^{n}$ y no solamente con la parte de este vector correspondiente a cada ecuación de conservación en particular.

\subsubsection{Discretización en el espacio: FEM Galerkin.}

En las expresiones (3.16) o, lo que es lo mismo, (3.18) a (3.21), todas las funciones implicadas ya se han discretizado en el tiempo, pero no en el espacio. Los vectores $\boldsymbol{U}, \boldsymbol{F}_{i}, \boldsymbol{Q}$ y $\boldsymbol{G}_{i}$, siguen siendo funciones continuas de la posición, lo que significa que, por ejemplo:

$$
\boldsymbol{F}_{i}{ }^{n}=\boldsymbol{F}_{i}{ }^{n}\left(x_{i}\right)
$$

Además, los incrementos temporales que aparecieron son también funciones de la posición, lo que significa tener, por ejemplo:

$$
\Delta \boldsymbol{F}_{i}^{n+1}=\Delta \boldsymbol{F}_{i}^{n+1}\left(x_{i}\right)=\boldsymbol{F}_{i}^{n+1}\left(x_{i}\right)-\boldsymbol{F}_{i}^{n}\left(x_{i}\right)
$$

Para discretizar en el espacio el dominio continuo, se utiliza el FEM, que consiste en dividir el dominio en elementos. Las variables de campo se aproximan dentro de los elementos, a través de polinomios que interpolan los valores de estas variables a partir de sus valores en los nodos de los elementos.

En este trabajo se utilizan elementos trilineales hexaédricos de ocho nodos. Por lo tanto, se tienen ocho funciones de interpolación, que constituyen la siguiente matriz:

$$
[\Phi]=\left[\begin{array}{llllllll}
\Phi_{1} & \Phi_{2} & \Phi_{3} & \Phi_{4} & \Phi_{5} & \Phi_{6} & \Phi_{7} & \Phi_{8}
\end{array}\right]
$$

Después de la discretización del dominio, las variables aproximadas obtenidas por interpolación se representan mediante las siguientes expresiones:

$$
\begin{aligned}
\widehat{\boldsymbol{U}}^{n} & =[\Phi]\{\boldsymbol{U}\}^{n} \\
\widehat{\boldsymbol{F}}_{i}{ }^{n} & =[\Phi]\left\{\boldsymbol{F}_{i}\right\}^{n} \\
\widehat{\boldsymbol{Q}}^{n} & =[\Phi]\{\boldsymbol{Q}\}^{n}
\end{aligned}
$$

En estas expresiones, $\{\boldsymbol{U}\}^{n},\{\boldsymbol{F}\}^{n}$ y $\{\boldsymbol{Q}\}^{n}$ son vectores de valores nodales. Por ejemplo, para el vector de variables de campo se tiene: 


$$
\{\boldsymbol{U}\}^{n}=\left\{\begin{array}{c}
\{\rho\} \\
\left\{\rho v_{1}\right\} \\
\left\{\rho v_{2}\right\} \\
\left\{\rho v_{3}\right\} \\
\{\rho e\} \\
\{\rho \tilde{v}\}
\end{array}\right\}
$$

donde, por ejemplo, el vector $\left\{\rho v_{1}\right\}^{n}$ contiene los productos de los valores de $\rho$ y $v_{1}$ en cada uno de los ocho nodos del elemento, es decir.

$$
\left\{\rho v_{1}\right\}^{n}=\left\{\begin{array}{c}
\left(\rho v_{1}\right)_{1} \\
\left(\rho v_{1}\right)_{2} \\
\vdots \\
\left(\rho v_{1}\right)_{8}
\end{array}\right\}^{n}
$$

Los vectores restantes se expanden de la misma forma.

Cabe destacar el hecho de que los vectores $\widehat{\boldsymbol{U}}^{n}, \widehat{\boldsymbol{F}}_{i}{ }^{n}$ y $\widehat{\boldsymbol{Q}}^{n}$, que aparecen en el lado izquierdo de las expresiones (3.25), (3.26) y (3.27), contienen las variables aproximadas, formadas por los productos de las funciones de interpolación constituidas por polinomios conocidos, y los valores nodales de las variables que son las incógnitas del problema a determinar. Sin embargo, el vector $\widehat{\boldsymbol{G}}_{i}{ }^{n}$ no ha aparecido aquí, dado que estos términos necesitan un tratamiento especial.

Una vez realizada la discretización, es necesario adoptar un método que permita establecer ecuaciones para determinar los valores nodales de las variables, de modo que se minimice la diferencia entre los valores de las variables aproximadas por interpolación y los valores de la solución exacta para las variables que fueron las incógnitas originales en el modelo matemático continuo.

Si en el sistema de ecuaciones dado por la expresión (3.16), ya discretizada en el tiempo, se reemplazan las variables incógnitas por las variables aproximadas (dado que, precisamente, estas últimas no son la solución exacta del sistema) las ecuaciones no serán satisfechas por esta solución aproximada, dejando un residuo para cada ecuación.

En el contexto del FEM, el método de los residuos ponderados consiste en minimizar el residuo que surge de reemplazar las variables incógnitas en el sistema (3.16) por las funciones aproximadas en el elemento.

Entre los métodos de residuos ponderados que se pueden utilizar, en el método de Bubnov-Galerkin se pondera al residuo utilizando las mismas funciones de interpolación utilizadas para interpolar las variables. Luego se minimiza al residuo, así ponderado, 
exigiendo que el producto interno del residuo y las funciones de interpolación sea nulo, es decir:

$$
\int_{\Omega}[\Phi]^{T} \boldsymbol{R} d \Omega=\{0\}
$$

donde $\boldsymbol{R}$ contiene los residuos de las ecuaciones, dados por:

$$
\begin{aligned}
\boldsymbol{R}= & \Delta \widehat{\boldsymbol{U}}_{I+1}^{n+1}-\Delta t\left[-\frac{\partial \widehat{\boldsymbol{F}}_{i}^{n}}{\partial x_{i}}-\frac{\partial \widehat{\boldsymbol{G}}_{i}{ }^{n}}{\partial x_{i}}-\widehat{\boldsymbol{Q}}^{n}+\frac{\Delta t}{2} \frac{\partial}{\partial x_{k}}\left(\widehat{\boldsymbol{A}}_{k}{ }^{n} \frac{\partial \widehat{\boldsymbol{F}}_{i}^{n}}{\partial x_{i}}+\widehat{\boldsymbol{Q}}^{n}\right)\right] \\
& +\frac{\Delta t}{2}\left[-\frac{\partial \Delta \widehat{\boldsymbol{F}}_{i_{I}}^{n+1}}{\partial x_{i}}-\frac{\partial \Delta \widehat{\boldsymbol{G}}_{i_{I}}^{n+1}}{\partial x_{i}}-\Delta \widehat{\boldsymbol{Q}}_{I}^{n+1}+\frac{\Delta t}{2} \frac{\partial}{\partial x_{k}}\left(\widehat{\boldsymbol{A}}_{k}{ }^{n} \frac{\partial \Delta \widehat{\boldsymbol{F}}_{i_{I}}^{n+1}}{\partial x_{i}}\right)\right]
\end{aligned}
$$

Así, resolviendo las ecuaciones (3.30) para los valores nodales de las variables de campo, se obtiene la solución del problema discretizado, ya que estos valores son precisamente los que minimizan al residuo y, por lo tanto, mejor aproximan a la solución exacta con las funciones de interpolación que fueron previamente definidas.

Como se mencionó anteriormente, los términos difusivos contenidos en el vector $\boldsymbol{G}_{i}^{n}$ no pueden ser interpolados en forma directa, como se muestra a continuación:

$$
\widehat{\boldsymbol{G}}_{i}^{n}=[\Phi]\left\{\boldsymbol{G}_{i}\right\}^{n}
$$

La expresión (3.32) no se puede utilizar directamente porque esto implicaría tener los valores nodales $\left\{\boldsymbol{G}_{i}\right\}^{n}$ del vector $\boldsymbol{G}_{i}^{n}$ y, dado que los términos difusivos y de conductividad térmica contienen derivadas segundas de las variables de campo, estos valores nodales no se pueden obtener cuando el elemento utilizado es lineal (como ocurre en este trabajo). Por lo tanto, es necesario integrar por partes utilizando el Teorema de Gauss-Green, lo cual permite disminuir el orden de las derivadas dentro del integrando.

Por otra parte, al aplicar el Teorema de Gauss-Green y resolver las integrales del tipo (3.30) para los términos $\boldsymbol{G}_{i}^{n}$, resulta obligado trabajar con las ecuaciones por separado usando expresiones del tipo de las (3.18) a (3.21) y ya no se puede manejar una única expresión vectorial del tipo de la (3.16), dado que las matrices diferirán dependiendo de la ecuación.

Entonces, aplicando el método clásico de Bubnov-Galerkin a las expresiones (3.18) a (3.21) en el contexto del método de los elementos finitos, aplicando el teorema de GaussGreen a los términos con derivadas segundas y, adoptando las siguientes abreviaciones de notación: $\left\{F_{i}^{M}\right\}=\left\{\rho v_{i}\right\} ;\left\{F_{i j}^{C M}\right\}=\left\{\rho v_{i} v_{j}+p \delta_{i j}\right\} ;\left\{F_{i}^{E}\right\}=\left\{(\rho e+p) v_{i}\right\} ;\left\{F_{i}^{S A}\right\}=\left\{\rho v_{i} \tilde{v}\right\}$, se 
obtienen las siguientes ecuaciones matriciales (ver Burbridge \& Awruch [29]; ver Anexo I) en donde se han omitido algunos términos de contorno que son despreciables:

Ecuación de conservación de la masa:

$$
\begin{aligned}
\{\Delta \rho\}_{I+1}^{n+1}= & \Delta t\left[M_{D}\right]^{-1}\left(-\left[B_{i}\right]\left\{F_{i}^{M}\right\}^{n}-\left[\alpha_{i j}\right]\left\{F_{i j}^{C M}\right\}^{n}\right) \\
& +\frac{\Delta t}{2}\left[M_{D}\right]^{-1}\left(-\left[B_{i}\right]\left\{\Delta F_{i}^{M}\right\}_{I}^{n+1}-\left[\alpha_{i j}\right]\left\{\Delta F_{i j}^{C M}\right\}_{I}^{n+1}\right)
\end{aligned}
$$

Ecuaciones de conservación de cantidad de movimiento:

$$
\begin{aligned}
\left\{\Delta \rho v_{j}\right\}_{I+1}^{n+1}=\Delta t\left[M_{D}\right]^{-1}( & -\left[B C_{i}\right]\left\{F_{i j}^{C M}\right\}^{n}-\left[A_{i j}^{P}\right]\left\{v_{i}\right\}^{n}-\left[A_{j}^{V}\right]\{p\}^{n} \\
& \left.-\left[D_{i j}\right]\left\{v_{i}\right\}^{n}+\left\{f_{j}\right\}^{n}\right) \\
+\frac{\Delta t}{2}\left[M_{D}\right]^{-1}( & -\left[B C_{i}\right]\left\{\Delta F_{i j}^{C M}\right\}_{I}^{n+1}-\left[A_{i j}^{P}\right]\left\{\Delta v_{i}\right\}_{I}^{n+1}-\left[A_{j}^{V}\right]\{\Delta p\}_{I}^{n+1} \\
& \left.-\left[D_{i j}\right]\left\{\Delta v_{i}\right\}_{I}^{n+1}\right)
\end{aligned}
$$

Ecuación de conservación de energía:

$$
\begin{gathered}
\{\Delta \rho e\}_{I+1}^{n+1}=\Delta t\left[M_{D}\right]^{-1}\left(-\left[B C_{i}\right]\left\{F_{i}^{E}\right\}^{n}-\left[A_{i}^{\bar{\theta}}\right]\left\{F_{i}^{M}\right\}^{n}-\left[A^{\bar{\varepsilon}}\right]\{p\}^{n}\right. \\
\left.-\left[E_{i}\right]\left\{v_{i}\right\}^{n}-[K]\{u\}^{n}+\{q\}^{n}\right) \\
+\frac{\Delta t}{2}\left[M_{D}\right]^{-1}\left(-\left[B C_{i}\right]\left\{\Delta F_{i}^{E}\right\}_{I}^{n+1}-\left[A_{i}^{\bar{\theta}}\right]\left\{\Delta F_{i}^{M}\right\}_{I}^{n+1}-\left[A^{\bar{\varepsilon}}\right]\{\Delta p\}_{I}^{n+1}\right. \\
\left.-\left[E_{i}\right]\left\{\Delta v_{i}\right\}_{I}^{n+1}-[K]\{\Delta u\}_{I}^{n+1}\right)
\end{gathered}
$$

Ecuación de transporte de $\tilde{v}(\mathrm{SA})$ :

$$
\begin{aligned}
\{\Delta \rho \tilde{v}\}_{I+1}^{n+1}=\Delta t\left[M_{D}\right]^{-1}( & -\left[B C_{i}\right]\left\{F_{i}^{S A}\right\}^{n}-\left[A^{\tilde{v}}\right]\{p\}^{n} \\
& \left.-[M]\{Q\}^{n}+\left[L_{i}\right]\left\{Q v_{i}\right\}^{n}-\left[D_{\tilde{v}}\right]\{\tilde{v}\}^{n}\right) \\
+\frac{\Delta t}{2}\left[M_{D}\right]^{-1} & \left(-\left[B C_{i}\right]\left\{\Delta F_{i}^{S A}\right\}_{I}^{n+1}-\left[A^{\tilde{v}}\right]\{p\}^{n}\right. \\
& \left.-[M]\{\Delta Q\}_{I}^{n+1}-\left[D_{\tilde{v}}\right]\{\Delta \tilde{v}\}_{I}^{n+1}\right)
\end{aligned}
$$

Siendo:

$$
\begin{gathered}
{\left[B_{i}\right]=\int_{\Omega_{E}}[\phi]^{T} \frac{\partial[\phi]}{\partial x_{i}} d \Omega ;\left[L_{i}\right]=\frac{\Delta t}{2}\left[B_{i}\right] ; \quad\left[\alpha_{i j}\right]=\frac{\Delta t}{2} \int_{\Omega_{E}} \frac{\partial[\phi]^{T}}{\partial x_{i}} \frac{\partial[\phi]}{\partial x_{j}} d \Omega ;} \\
{\left[C_{i}\right]=\frac{\Delta t}{2} \int_{\Omega_{E}}\left([\phi]\left\{v_{k}\right\}^{n}\right) \frac{\partial[\phi]^{T}}{\partial x_{k}} \frac{\partial[\phi]}{\partial x_{i}} d \Omega ;} \\
{\left[A_{i j}^{P}\right]=\frac{\Delta t}{2} \int_{\Omega_{E}}\left([\phi]\{p\}^{n}\right) \frac{\partial[\phi]^{T}}{\partial x_{i}} \frac{\partial[\phi]}{\partial x_{j}} d \Omega ;\left[A_{j}^{V}\right]=\frac{\Delta t}{2} \int_{\Omega_{E}}\left([\phi]\left\{v_{j}\right\}^{n}\right) \frac{\partial[\phi]^{T}}{\partial x_{i}} \frac{\partial[\phi]}{\partial x_{i}} d \Omega ;}
\end{gathered}
$$




$$
\begin{aligned}
& {\left[A^{\bar{\varepsilon}}\right]=\frac{\Delta t}{2} \int_{\Omega_{E}}\left([\phi]\{\bar{\varepsilon}\}^{n}\right) \frac{\partial[\phi]^{T}}{\partial x_{i}} \frac{\partial[\phi]}{\partial x_{i}} d \Omega ; \bar{\varepsilon}=\gamma e-\bar{\theta} ; \bar{\theta}=(\gamma-1) \frac{|\boldsymbol{V}|^{2}}{2}} \\
& {\left[A_{i}^{\bar{\theta}}\right]=\frac{\Delta t}{2} \int_{\Omega_{E}}\left([\phi]\left\{\bar{\theta} v_{k}\right\}^{n}\right) \frac{\partial[\phi]^{T}}{\partial x_{k}} \frac{\partial[\phi]}{\partial x_{i}} d \Omega ; \quad\left[A^{\widetilde{v}}\right]=\frac{\Delta t}{2} \int_{\Omega_{E}}\left([\phi]\{\tilde{v}\}^{n}\right) \frac{\partial[\phi]^{T}}{\partial x_{i}} \frac{\partial[\phi]}{\partial x_{i}} d \Omega} \\
& {\left[B C_{i}\right]=\left[B_{i}\right]+\left[C_{i}\right] ;} \\
& {\left[D_{i j}\right]=\left\{\begin{array}{l}
\int_{\Omega_{E}} \eta\left(2+\frac{\lambda}{\eta}\right) \frac{\partial[\phi]^{T}}{\partial x_{i}} \frac{\partial[\phi]}{\partial x_{(i)}} d \Omega+\int_{\Omega_{E}} \eta \frac{\partial[\phi]^{T}}{\partial x_{k}} \frac{\partial[\phi]}{\partial x_{k}} d \Omega, \text { si } i=j \mathrm{y}:\left\{\begin{array}{l}
i=1 \rightarrow k=2,3 \\
i=2 \rightarrow k=1,3 \\
i=3 \rightarrow k=1,2
\end{array}\right. \\
\int_{\Omega_{E}} \eta \frac{\partial[\phi]^{T}}{\partial x_{i}} \frac{\partial[\phi]}{\partial x_{j}} d \Omega+\int_{\Omega_{E}} \lambda \frac{\partial[\phi]^{T}}{\partial x_{j}} \frac{\partial[\phi]}{\partial x_{i}} d \Omega, \quad \text { si } i \neq j
\end{array}\right.} \\
& \eta=\mu+\mu_{t} ; \lambda=-\frac{2}{3} \eta \\
& {\left[E_{i}\right]=\int_{\Omega_{E}}\left[\eta\left([\phi]\left\{v_{i}\right\}^{n}\right) \frac{\partial[\phi]^{T}}{\partial x_{k}} \frac{\partial[\phi]}{\partial x_{k}}+\eta\left([\phi]\left\{v_{k}\right\}^{n}\right) \frac{\partial[\phi]^{T}}{\partial x_{i}} \frac{\partial[\phi]}{\partial x_{k}}\right] d \Omega} \\
& +\int_{\Omega_{E}} \lambda\left([\phi]\left\{v_{k}\right\}^{n}\right) \frac{\partial[\phi]^{T}}{\partial x_{k}} \frac{\partial[\phi]}{\partial x_{i}} d \Omega \\
& {[K]=\int_{\Omega_{E}} \kappa \frac{\partial[\phi]^{T}}{\partial x_{i}} \frac{\partial[\phi]}{\partial x_{i}} d \Omega ; \quad \kappa=K+K_{t}} \\
& {[M]=\int_{\Omega_{E}}[\phi]^{T}[\phi] d \Omega} \\
& {\left[M_{D}\right]=\left\{\begin{array}{lr}
\frac{\Omega_{E}}{8} & \text { para los términos de la diagonal ppal. } \\
0 & \text { para todos los términos fuera de la diagonal ppal. }
\end{array}\right.} \\
& {\left[D_{\tilde{v}}\right]=\int_{\Omega_{E}} \frac{1}{\sigma}\left[\mu+\left([\phi]\{\rho \tilde{v}\}^{n}\right)\right] \frac{\partial[\phi]^{T}}{\partial x_{i}} \frac{\partial[\phi]}{\partial x_{i}} d \Omega} \\
& \{Q\}^{n}=-c b_{1}\left(S^{n}\right)_{E}\{\rho \tilde{v}\}^{n}+c w_{1}\left(f_{w}{ }^{n}\right)_{E}\left\{\rho\left(\frac{\tilde{v}}{d}\right)^{2}\right\}^{n}-\frac{c b_{2}}{\sigma}\left(\rho^{n}\right)_{E}\left(\frac{\partial \tilde{v}^{n}}{\partial x_{i}}\right)_{E}\left(\frac{\partial \tilde{v}^{n}}{\partial x_{i}}\right)_{E}\{I\} \\
& +\frac{1}{\sigma}\left[\left(v^{n}\right)_{E}+\left(\tilde{v}^{n}\right)_{E}\right]\left(\frac{\partial \rho^{n}}{\partial x_{i}}\right)_{E}\left(\frac{\partial \tilde{v}^{n}}{\partial x_{i}}\right)_{E}\{I\}
\end{aligned}
$$




$$
\begin{gathered}
\left\{Q v_{i}\right\}^{n}=-c b_{1}\left(S^{n}\right)_{E}\left\{\rho \tilde{v} v_{i}\right\}^{n}+c w_{1}\left(f_{w}{ }^{n}\right)_{E}\left\{\rho\left(\frac{\tilde{v}}{d}\right)^{2} v_{i}\right\}^{n} \\
-\frac{c b_{2}}{\sigma}\left(\rho^{n}\right)_{E}\left(\frac{\partial \tilde{v}^{n}}{\partial x_{i}}\right)_{E}\left(\frac{\partial \tilde{v}^{n}}{\partial x_{i}}\right)_{E}\left\{v_{i}\right\} \\
+\frac{1}{\sigma}\left[\left(v^{n}\right)_{E}+\left(\tilde{v}^{n}\right)_{E}\right]\left(\frac{\partial \rho^{n}}{\partial x_{i}}\right)_{E}\left(\frac{\partial \tilde{v}^{n}}{\partial x_{i}}\right)_{E}\left\{v_{i}\right\} \\
\left\{f_{j}\right\}^{n}=\int_{\Gamma_{E}}\left[\Phi^{*}\right]^{T}\left[\eta\left(\frac{\partial[\phi]}{\partial x_{i}}\left\{v_{j}\right\}^{n}+\frac{\partial[\phi]}{\partial x_{j}}\left\{v_{i}\right\}^{n}\right)+\lambda\left(\frac{\partial[\phi]}{\partial x_{k}}\left\{v_{k}\right\}^{n}\right) \delta_{i j}\right] n_{i} d \Gamma \\
\{q\}^{n}=\int_{\Gamma_{E}}\left[\Phi^{*}\right]^{T}\left([\Phi]\left\{v_{j}\right\}^{n}\right)\left[\eta\left(\frac{\partial[\phi]}{\partial x_{i}}\left\{v_{j}\right\}^{n}+\frac{\partial[\phi]}{\partial x_{j}}\left\{v_{i}\right\}^{n}\right)+\lambda\left(\frac{\partial[\phi]}{\partial x_{k}}\left\{v_{k}\right\}^{n}\right) \delta_{i j}\right] n_{i} d \Gamma \\
+\int_{\Gamma_{E}}\left[\Phi^{*}\right]^{T} \kappa\left(\frac{\partial[\phi]}{\partial x_{i}}\{u\}^{n}\right) n_{i} d \Gamma
\end{gathered}
$$

En estas expresiones $\Omega_{E}$ y $\Gamma_{E}$ son el volumen del elemento y su superficie de contorno respectivamente, las expresiones con subíndice $E$ son promedios de elemento, $[\Phi]=\left[\Phi_{1}, \Phi_{2}\right.$, $\left.\ldots \Phi_{8}\right]$ es la matriz fila que contiene a las funciones de interpolación para cada nodo local, $\left[\Phi^{*}\right]$ es la matriz fila que contiene las funciones de interpolación evaluadas en el contorno de las superficie, $[M]$ es la matriz de masa consistente $\left[M_{D}\right]$ es la matriz de masa concentrada y $\{I\}^{T}=\left\{\begin{array}{llllllll}1 & 1 & 1 & 1 & 1 & 1 & 1 & 1\end{array}\right\}$. Los términos que involucran la energía cinética turbulenta han sido despreciados. También se despreciaron los términos de contorno que aparecen en la ecuación de transporte de Spalart-Allmaras y en los términos iterativos de todas las ecuaciones.

Como se dijo en apartado anterior, el esquema de avance en el tiempo dado por la expresión (3.15) es, en primera instancia, implícito. En principio, esto implica que, al aplicar el método de Bubnov-Galerkin para obtener una expresión de recurrencia para $\Delta \boldsymbol{U}^{n+1}$, deberían pasarse al lado izquierdo de la ecuación todos los términos definidos en $n+1$, resultando, por lo tanto, imposible obtener un sistema de ecuaciones desacopladas. Si esto fuera así, debería resolverse un sistema de ecuaciones algebraicas acopladas a cada paso de tiempo.

Sin embargo, la forma de la expresión (3.15), obtenida al elegir los parámetros $S_{1}=1 / 2$ y $S_{2}=1 / 2$, permite resolver los términos del segundo corchete de (3.15) en forma iterativa como se indicó en las expresiones (3.16) a (3.21), con lo cual en el lado izquierdo de las ecuaciones ya discretizadas resulta únicamente la matriz de masa consistente multiplicada por los incrementos $\Delta \boldsymbol{U}^{n+1}$. Esto permite reemplazar a la matriz de masa consistente por la matriz de masa concentrada que, al ser diagonal, puede pasar directamente al miembro derecho de la 
ecuación, simplemente dividiendo cada ecuación por cada uno de los elementos de la matriz de masa concentrada, resultado un sistema de ecuaciones desacopladas. Esto se indica en las expresiones (3.33) a (3.36) con la matriz de masa concentrada invertida en los miembros derechos de dichas ecuaciones.

Entonces, resuelto de esta forma, este esquema de Taylor-Galerkin expresado en las ecuaciones matriciales (3.33) a (3.36) constituye un esquema explícito que resulta con-

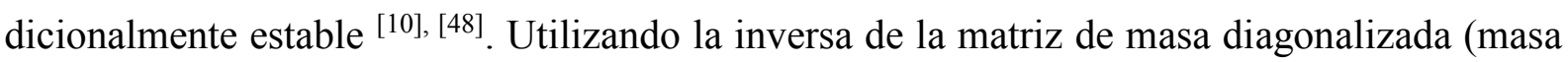
concentrada) no se requiere la inversión de la matriz de masa consistente.

Una vez ensambladas estas ecuaciones para todos los elementos de la malla y aplicadas las correspondientes condiciones de contorno, los valores nodales de $\rho, \rho v_{j}$, $\rho e y \rho \tilde{v}$ se pueden calcular, a cada paso de tiempo, usando un esquema iterativo para los términos en $n+1$ del lado derecho de las ecuaciones. Sin embargo, esta iteración sólo resulta necesaria durante pocos pasos de tiempo, por lo cual no resulta onerosa en términos de tiempo de cómputo ${ }^{[3]}$.

Entonces, los valores nodales de las componentes de velocidad, energía total específica y de la variable $\tilde{v}$ pueden obtenerse inmediatamente. Luego se calculan los valores nodales de la energía interna específica y, utilizando la ecuación de estado, los valores nodales de presión. Para casos no viscosos, donde los términos viscosos no son tenidos en cuenta, se omiten los términos que contienen a las matrices $\left[D_{i j}\right],\left[E_{i}\right]$ y $[K]$, los vectores $\left\{f_{j}\right\}^{n}$ y $\{q\}^{n}$ y la ecuación de transporte para el modelo de turbulencia de SA completa.

Considerando que este algoritmo es explícito, el mismo resulta condicionalmente estable. La condición de estabilidad de Courant-Friedrichs-Lewy (CFL) para cada elemento, en forma adimensional, viene dada por:

$$
\Delta t_{E}=\beta \frac{L_{E}}{(M+1) c}
$$

donde $L_{E}$ es la longitud característica adimensional del elemento, $c$ es la velocidad del sonido adimensional promedio de elemento, $M$ es el Mach local en el elemento y $\beta$ es un coeficiente de seguridad cuyos valores se encuentran en el intervalo $0.2 \leq \beta \leq 0.7$.

\subsection{EL MÉTODO CBS.}

\subsubsection{Método CBS - Split A.}

El algoritmo CBS para flujo compresible consiste en cuatro pasos (ver Zienkiewicz \& Codina ${ }^{[17]}$ ). En el primer paso se calculan valores intermedios de las variables conservativas o 
variables de flujo de las ecuaciones de cantidad de movimiento omitiendo los términos que contienen gradientes de presión. En el segundo paso se resuelve la ecuación de continuidad para obtener los incrementos de densidad en el fluido. En el tercer paso se actualizan las variables de flujo de las ecuaciones de cantidad de movimiento. Finalmente, en el cuarto paso se resuelve la ecuación de la energía y de transporte de SA y se calcula la presión usando la ecuación de estado. Una vez planteada la discretización en el tiempo propuesta por el método CBS - Split $A$ se obtienen las siguientes ecuaciones:

Paso 1: Resolución de las variables de flujo de cantidad de movimiento intermedias:

$$
\Delta\left(\rho v_{j}\right)_{*}^{n+1}=\Delta t\left[-\frac{\partial\left(\rho v_{j} v_{i}\right)^{n}}{\partial x_{i}}+\frac{\partial \tau_{i j}^{n}}{\partial x_{i}}+\frac{\Delta t}{2} v_{k}^{n} \frac{\partial}{\partial x_{k}}\left(\frac{\partial\left(\rho v_{j} v_{i}\right)^{n}}{\partial x_{i}}\right)\right]
$$

Paso 2: Resolución de la ecuación de continuidad:

$$
\Delta \rho^{n+1}=\Delta t\left[-\frac{\partial\left(\rho v_{i}\right)^{n}}{\partial x_{i}}-\frac{1}{2} \frac{\partial \Delta\left(\rho v_{j}\right)_{*}^{n+1}}{\partial x_{k}}+\frac{\Delta t}{2} \frac{\partial}{\partial x_{i}}\left(\frac{\partial p^{n}}{\partial x_{i}}\right)\right]
$$

Paso 3: Calculo de la corrección de variables de flujo de cantidad de movimiento:

$$
\Delta\left(\rho v_{j}\right)^{n+1}=\Delta\left(\rho v_{j}\right)_{*}^{n+1}+\Delta t\left[-\frac{\partial p^{n}}{\partial x_{j}}+\frac{\Delta t}{2} v_{k}^{n} \frac{\partial}{\partial x_{k}}\left(\frac{\partial p^{n}}{\partial x_{i}}\right)\right]
$$

Paso 4: Resolución de la ecuación de la energía y ecuación de transporte de $\tilde{v}$ (SA):

$$
\begin{gathered}
\Delta(\rho e)^{n+1}=\Delta t\left[-\frac{\partial\left[(\rho e+p) v_{i}\right]^{n}}{\partial x_{i}}+\frac{\partial\left(\tau_{i j} v_{j}\right)^{n}}{\partial x_{i}}+\frac{\partial \psi_{i}{ }^{n}}{\partial x_{i}}-\frac{\partial q_{i}{ }^{n}}{\partial x_{i}}\right. \\
\left.+\frac{\Delta t}{2} v_{k}{ }^{n} \frac{\partial}{\partial x_{k}}\left(\frac{\partial\left[(\rho e+p) v_{i}\right]^{n}}{\partial x_{i}}\right)\right] \\
\Delta(\rho \tilde{v})^{n+1}=\Delta t\left[-\frac{\partial\left(\rho \tilde{v} v_{i}\right)^{n}}{\partial x_{i}}+\frac{\partial \varphi_{i}^{n}}{\partial x_{i}}-P^{n}-D^{n}-D_{T}^{n}\right. \\
\left.+\frac{\Delta t}{2} v_{k}^{n} \frac{\partial}{\partial x_{k}}\left(\frac{\partial\left(\rho \tilde{v} v_{i}\right)^{n}}{\partial x_{i}}+P^{n}+D^{n}+D_{T}^{n}\right)\right]
\end{gathered}
$$

Las expresiones anteriores constituyen la versión explícita del método CBS. Este método puede plantearse tanto en su versión explícita como en sus versiones implícita y semiimplicita ${ }^{[3]}$. En forma semejante al esquema TG, en el caso del método CBS esto depende de la selección de dos parámetros que, para este último método, se denominan $\theta_{1}$ y $\theta_{2}$ y que, en las expresiones (3.39) a (3.43), ya han sido previamente fijados en los valores $\theta_{1}=1 / 2$ y 
$\theta_{2}=0$ como para obtener la versión explícita. Esta versión es, entonces, condicionalmente estable por lo cual el paso de tiempo debe definirse teniendo en cuenta la expresión (3.38) al igual que con el esquema TG.

Una vez planteada la discretización temporal se procede a la discretización en el dominio espacial utilizando el método clásico de Bubnov-Galerkin. Los términos que involucran derivadas segundas de la posición en las expresiones aproximadas se integran por partes utilizando el teorema de Gauss-Green para relajar las exigencias sobre las funciones de interpolación en términos de continuidad en la frontera entre elementos. Una vez implementado este método se obtienen las siguientes expresiones:

Paso 1: Resolución de las variables de flujo de cantidad de movimiento intermedias

$$
\left\{\Delta \rho v_{j}\right\}_{*}^{n+1}=\Delta t_{\text {ext }}\left[M_{D}\right]^{-1}\left(-\left[B C H_{i}\right]\left\{\rho v_{j} v_{i}\right\}^{n}-\left[D_{i j}\right]\left\{v_{i}\right\}^{n}+\left\{f_{j}\right\}^{n}\right)
$$

Paso 2: Resolución de la ecuación de conservación de la masa:

$$
\{\Delta \rho\}^{n+1}=\Delta t_{\text {ext }}\left[M_{D}\right]^{-1}\left(-\left[B_{i}\right]\left\{F_{i}^{M}\right\}^{n}-\frac{1}{2}\left[B_{i}\right]\left\{\Delta \rho v_{i}\right\}_{*}^{n+1}-[P]\{p\}^{n}+\{h\}^{n}\right)
$$

Paso 3: Calculo de la corrección de variables de flujo de cantidad de movimiento

$$
\left\{\Delta \rho v_{j}\right\}^{n+1}=\left\{\Delta \rho v_{j}\right\}_{*}^{n+1}+\Delta t_{e x t}\left[M_{D}\right]^{-1}\left(-\left[B C H_{i}\right]\{p\}^{n}\right)
$$

Paso 4: Resolución de la ecuación de la energía y ecuación de transporte de $\tilde{v}$ (SA):

$$
\begin{gathered}
\{\Delta \rho e\}^{n+1}=\Delta t_{\text {ext }}\left[M_{D}\right]^{-1}\left(-\left[B C H_{i}\right]\left\{F_{i}^{E}\right\}^{n}-\left[E_{i}\right]\left\{v_{i}\right\}^{n}-[K]\{u\}^{n}+\{q\}^{n}\right) \\
\{\Delta \rho \tilde{v}\}^{n+1}=\Delta t_{\text {ext }}\left[M_{D}\right]^{-1}\left(-\left[B C H_{i}\right]\left\{F_{i}^{S A}\right\}^{n}-[M]\{Q\}^{n}+\left[L_{i}\right]\left\{Q v_{i}\right\}^{n}-\left[D_{\tilde{v}}\right]\{\tilde{v}\}^{n}\right)
\end{gathered}
$$

Las matrices $[M],\left[M_{D}\right],\left[D_{i j}\right],\left[E_{i}\right],\left[L_{i}\right],\left[D_{\widetilde{v}}\right]$ y $[K]$ y los vectores $\left\{f_{j}\right\}^{\mathrm{n}}$ y $\{q\}^{\mathrm{n}}$ son los mismos que los obtenidos con el esquema TG. Sin embargo, para el algoritmo CBS aquí implementado, aparecen las siguientes matrices y vectores que deben considerarse:

$$
\begin{gathered}
{[P]=\frac{\Delta t_{\text {in }}}{2} \int_{\Omega_{E}} \frac{\partial[\phi]^{T}}{\partial x_{i}} \frac{\partial[\phi]}{\partial x_{i}} d \Omega=\left[\alpha_{i i}\right] ;\{h\}^{n}=\frac{\Delta t_{i n}}{2} \int_{\Gamma_{E}}\left[\phi^{*}\right]^{T}\left(\frac{\partial[\phi]}{\partial x_{i}}\{p\}^{n}\right) n_{i} d \Gamma} \\
{\left[B C H_{i}\right]=\left[B_{i}\right]+\left(\left[C_{i}\right]+\left[H_{i}\right]\right)} \\
{\left[H_{i}\right]=\frac{\Delta t_{\text {in }}}{2} \int_{\Omega_{E}}\left[\frac{\partial[\phi]}{\partial x_{i}}\left\{v_{k}\right\}^{n}\right][\phi]^{T} \frac{\partial[\phi]}{\partial x_{i}} d \Omega}
\end{gathered}
$$

en donde las matrices $\left[B_{i}\right]$ y $\left[C_{i}\right]$ son las que ya se tenían en (3.37). 
Las ecuaciones fueron resueltas, en primera instancia, con un valor uniforme de $\Delta t$ en toda la malla de elementos finitos, adoptando el menor valor entre todos los $\Delta t_{E}$ obtenidos aplicando la expresión (3.38). Sin embargo, algunos investigadores ${ }^{[23]}$ han determinado que la utilización del $\Delta t$ local en los términos estabilizadores del método CBS (aquellos que contienen derivadas segundas de las variables de flujo o de la presión) proporciona mejor estabilización de las aproximaciones espaciales. Por lo tanto, al formar las matrices $\left[\mathrm{BCH}_{i}\right] \mathrm{y}$ $[P]$ y el vector $\{h\}^{\mathrm{n}}$ se utiliza un paso de tiempo local $\Delta t=\Delta t_{i n}$.

El paso de tiempo que domina el avance en el tiempo es el externo $\left(\Delta t_{\text {ext }}\right)$, el que aparece en las ecuaciones (3.44) a (3.48), mientras que el paso de tiempo interno $\left(\Delta t_{\text {in }}\right)$ es un valor que sólo afecta a ciertas matrices y vectores en las ecuaciones de elemento y puede adaptarse para que sea tal que la convergencia general del método CBS se vea mejorada.

Efectivamente, en el trabajo antes mencionado se muestra claramente que el método CBS con paso de tiempo interno presenta una mejora de convergencia cuando se lo compara con el mismo método CBS con paso de tiempo único, y con el método TG.

\subsubsection{Método CBS - “single-step”.}

Una variante del método CBS que implica cierta simplificación adicional permite eliminar la operación de "Split" de cantidad de movimiento de manera de obtener un algoritmo de un único paso. Los autores (ver Zienkiewicz et al. ${ }^{[3]}$ ) señalan que esta variante permite ahorrar tiempo de cómputo con relación al método CBS, pero sugieren no utilizarlo para altos números de Mach.

Una vez realizada la discretización temporal que surge de hacer las simplificaciones sobre el CBS (con los valores $\theta_{1}=1 / 2$ y $\theta_{2}=0$ ), las ecuaciones resultan:

Ecuación de conservación de masa:

$$
\Delta \rho^{n+1}=\Delta t\left[-\frac{\partial\left(\rho v_{i}\right)^{n}}{\partial x_{i}}+\frac{\Delta t}{2} \frac{\partial}{\partial x_{i}}\left(\frac{\partial p^{n}}{\partial x_{i}}\right)\right]
$$

Ecuación de conservación de cantidad de movimiento:

$$
\Delta \rho v_{j}^{n+1}=\Delta t\left[-\frac{\partial\left(\rho v_{j} v_{i}+p \delta_{i j}\right)^{n}}{\partial x_{i}}+\frac{\partial \tau_{i j}^{n}}{\partial x_{i}}+\frac{\Delta t}{2} v_{k}^{n} \frac{\partial}{\partial x_{k}}\left(\frac{\partial\left(\rho v_{j} v_{i}+p \delta_{i j}\right)^{n}}{\partial x_{i}}\right)\right]
$$


Ecuación de conservación de energía:

$$
\begin{aligned}
\Delta \rho e^{n+1}=\Delta t & {\left[-\frac{\partial\left[(\rho e+p) v_{i}\right]^{n}}{\partial x_{i}}+\frac{\partial\left(\tau_{i j} v_{j}\right)^{n}}{\partial x_{i}}+\frac{\partial \psi_{i}{ }^{n}}{\partial x_{i}}-\frac{\partial q_{i}{ }^{n}}{\partial x_{i}}\right.} \\
& \left.+\frac{\Delta t}{2} v_{k}{ }^{n} \frac{\partial}{\partial x_{k}}\left(\frac{\partial\left[(\rho e+p) v_{i}\right]^{n}}{\partial x_{i}}\right)\right]
\end{aligned}
$$

Ecuación de transporte de $\tilde{v}(\mathrm{SA})$ :

$$
\begin{aligned}
\Delta \rho \tilde{v}^{n+1} & =\Delta t\left[-\frac{\partial\left(\rho \tilde{v} v_{i}\right)^{n}}{\partial x_{i}}+\frac{\partial \varphi_{i}{ }^{n}}{\partial x_{i}}-P^{n}-D^{n}-D_{T}^{n}\right. \\
& \left.+\frac{\Delta t}{2} v_{k}{ }^{n} \frac{\partial}{\partial x_{k}}\left(\frac{\partial\left(\rho \tilde{v} v_{i}\right)^{n}}{\partial x_{i}}+P^{n}+D^{n}+D_{T}^{n}\right)\right]
\end{aligned}
$$

Nuevamente, en la expresión (3.52) los términos donde aparece $\psi_{i}$ no se consideran, mientras que en la expresión (3.53) los valores de $\varphi_{i}, P, D$ y $D_{T}$ surgen de las expresiones (2.41) a (2.43). Una vez aplicado el método de Bubnov-Galerkin para la discretización espacial con el FEM, se obtienen las siguientes ecuaciones matriciales elementales:

Ecuación de conservación de la masa

$$
\{\Delta \rho\}^{n+1}=\Delta t_{\text {ext }}\left[M_{D}\right]^{-1}\left(-\left[B_{i}\right]\left\{F_{i}^{M}\right\}^{n}-[P]\{p\}^{n}-\{h\}^{n}\right)
$$

Ecuación de cantidad de movimiento

$$
\left\{\Delta \rho v_{j}\right\}^{n+1}=\Delta t_{e x t}\left[M_{D}\right]^{-1}\left(-\left[B C H_{i}\right]\left\{F_{i j}^{C M}\right\}^{n}-\left[D_{i j}\right]\left\{v_{i}\right\}^{n}+\left\{f_{j}\right\}^{n}\right)
$$

Ecuación de la energía

$$
\{\Delta \rho e\}^{n+1}=\Delta t_{\text {ext }}\left[M_{D}\right]^{-1}\left(-\left[B C H_{i}\right]\left\{F_{i}^{E}\right\}^{n}-\left[E_{i}\right]\left\{v_{i}\right\}^{n}-[K]\{u\}^{n}+\{q\}^{n}\right)
$$

Ecuación de transporte de $\tilde{v}$ (SA)

$$
\{\Delta \rho \tilde{v}\}^{n+1}=\Delta t_{\text {ext }}\left[M_{D}\right]^{-1}\left(-\left[B C H_{i}\right]\left\{F_{i}^{S A}\right\}^{n}-[M]\{Q\}^{n}+\left[L_{i}\right]\left\{Q v_{i}\right\}^{n}-\left[D_{\tilde{v}}\right]\{\tilde{v}\}^{n}\right)
$$

Siendo todas las matrices y vectores los mismos que ya fueron indicados anteriormente.

\subsection{ESQUEMA HÍBRIDO: TAYLOR-GALERKIN MODIFICADO (MTG: Modified Taylor-Galerkin).}

Se presenta aquí un esquema alternativo a partir de algunas observaciones realizadas en este trabajo. Así, el esquema híbrido aquí propuesto es novedoso, en el sentido que es consecuencia de los estudios realizados con los algoritmos ya conocidos, T-G y CBS, y de las propuestas que se detallan a continuación. 
En primer lugar, es importante señalar que los términos estabilizadores (aquellos afectados por la matriz Jacobiana de Euler $\boldsymbol{A}_{k}^{n}$ en el esquema TG y por $v_{k}^{n}$ en el método CBS) tienen como objeto, como ya se indicó, aportar cierto amortiguamiento para estabilizar las oscilaciones numéricas que surgen de aplicar Bubnov-Galerkin a los términos convectivos. Es por ello por lo que, a priori, estos términos podrán tener diferente forma, incluso una forma que no sea estrictamente derivada del desarrollo matemático formal, siempre y cuando se pueda determinar que la nueva forma adoptada para estos términos aporte la cantidad adecuada de amortiguamiento.

Comparando estos términos correspondientes a ambos esquemas, TG y CBS, se observa que son similares, difiriendo en que el método CBS la matriz está afectada por $v_{k}^{n} \mathrm{y}$ no por $\boldsymbol{A}_{k}^{n}$. Es decir, en el esquema TG tiene la forma siguiente:

$$
\frac{\Delta t}{2} \frac{\partial}{\partial x_{k}}\left(\boldsymbol{A}_{k}{ }^{n} \frac{\partial \boldsymbol{F}_{i}{ }^{n}}{\partial x_{i}}\right)
$$

mientras que en el método CBS tienen la forma genérica siguiente:

$$
\frac{\Delta t}{2} v_{k}{ }^{n} \frac{\partial}{\partial x_{k}}\left(\frac{\partial \boldsymbol{F}_{i}{ }^{n}}{\partial x_{i}}\right)
$$

En el capítulo cuatro se verá que tanto los valores de $\boldsymbol{A}_{k}^{n}$ en el esquema TG como los valores de $v_{k}^{n}$ en el método CBS serán adoptados constantes como parte del método de reducción parcial de las integrales que se utiliza para poder integrar analíticamente. Si dichos valores son tomados constantes, la diferencia entre tenerlos dentro de las derivadas respecto de $x_{k}$, o fuera de dichas derivadas, se desvanece (y la matriz $\left[H_{i}\right]$ del método CBS se anula). Esto último sugiere la idea de adoptar la siguiente simplificación:

$$
\boldsymbol{A}_{k}^{n}=v_{k}^{n} \boldsymbol{I}
$$

siendo $I$ la matriz identidad de $6 \times 6$, lo cual conduce a la siguiente expresión para los términos estabilizadores en el esquema TG:

$$
\frac{\Delta t}{2} \frac{\partial}{\partial x_{k}}\left(v_{k}^{n} \frac{\partial \boldsymbol{F}_{i}{ }^{n}}{\partial x_{i}}\right)
$$

De esta forma, para el esquema de integración que se utiliza en este trabajo, se incorpora exactamente la misma cantidad de amortiguamiento que en el método CBS con la evidente ganancia en simplificación de las ecuaciones (3.33) a (3.36) por la eliminación de los términos asociados a las matrices $\left[A_{i j}^{P}\right],\left[A_{j}^{V}\right],\left[A^{\bar{\varepsilon}}\right],\left[A_{i}^{\bar{\theta}}\right],\left[A^{\widetilde{v}}\right]$. El esquema que resulta de 
aplicar la simplificación (3.60) al esquema TG conserva las características principales de este esquema, y difiere del método $\mathrm{CBS}$ en que no se requiere la separación de variables característica de este último método. Además, otra diferencia importante con el esquema CBS está dada por la ecuación de continuidad, la cual se transforma y resulta en la siguiente expresión:

$$
\{\Delta \rho\}_{I+1}^{n+1}=\Delta t\left[M_{D}\right]^{-1}\left[\left(-\left[B C_{i}\right]\left\{F_{i}^{M}\right\}^{n}\right)+\frac{\Delta t}{2}\left(-\left[B C_{i}\right]\left\{\Delta F_{i}^{M}\right\}_{I}^{n+1}\right)\right]
$$

El sistema de ecuaciones resultante está compuesto por la ecuación (3.62) y las ecuaciones (3.34), (3.35) y (3.36) en donde se eliminan los términos que contienen a las matrices $\left[A_{i j}^{P}\right],\left[A_{j}^{V}\right],\left[A^{\bar{\varepsilon}}\right],\left[A_{i}^{\bar{\theta}}\right],\left[A^{\widetilde{v}}\right]$. El sistema completo y la forma de obtenerlo puede verse en la referencia [29]. En dicha referencia ya se utilizaba esta variante para el esquema TG y los resultados obtenidos en dicho trabajo mostraron que no existen diferencias apreciables entre usar la matriz $\boldsymbol{A}_{k}^{n}$ completa o utilizar $v_{k}^{n} \boldsymbol{I}$ para números de Mach mayores que uno. Aquí denominaremos a esta variante como esquema TG2.

Por otro lado, puede observarse que, si en la matriz Jacobiana de Euler $\boldsymbol{A}_{k}^{n}$ las derivadas del vector de flujo $\boldsymbol{F}_{i}$ con respecto al vector de las variables conservativas $\boldsymbol{U}$ se realizan componente a componente (correspondiente a cada ecuación de conservación separadamente) y se desconsideran los términos de presión, esta matriz resulta $\boldsymbol{A}_{k}^{n}=v_{k}^{n} \boldsymbol{I}$, como se propuso en (3.60).

La simplificación mencionada arriba pareciera introducir ciertas oscilaciones en zonas de muy baja velocidad, pero trae aparejada una considerable reducción de tiempo de cómputo. Además, durante el desarrollo de este trabajo resultó evidente que el principal problema de estabilización a resolver está dado en los valores de densidad y presión los cuales, fundamentalmente, están controlados por la ecuación de continuidad.

Con el fin de obtener un método igual de eficiente que el esquema TG2, pero con las propiedades de convergencia del método CBS, teniendo en cuenta la anterior observación, se propone ahora una modificación adicional al esquema TG2 que consiste en cambiar el término estabilizador en la ecuación de continuidad por una expresión similar, pero definida a partir de la presión, como la que se utiliza en el método CBS single-step.

Dado que el esquema TG2 presenta buena convergencia para Mach supersónicos, resulta conveniente plantear esta modificación únicamente en regiones donde el Mach local 


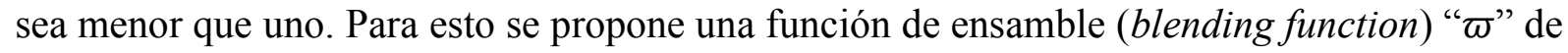
tal forma que:

$$
\begin{aligned}
& \Delta(\rho)_{I+1}^{n+1}=\Delta t\left\{-\frac{\partial\left(\rho v_{i}\right)^{n}}{\partial x_{i}}+\frac{\Delta t}{2}\left[\varpi \frac{\partial}{\partial x_{i}}\left(\frac{\partial p^{n}}{\partial x_{i}}\right)+(1-\varpi) \frac{\partial}{\partial x_{k}}\left(v_{k}{ }^{n} \frac{\partial\left(\rho v_{i}\right)^{n}}{\partial x_{i}}\right)\right]\right\} \\
& +\frac{\Delta t}{2}\left\{-\frac{\partial \Delta\left(\rho v_{i}\right)_{I}^{n+1}}{\partial x_{i}}+\frac{\Delta t}{2}\left[\varpi \frac{\partial}{\partial x_{i}}\left(\frac{\partial \Delta p_{I}^{n+1}}{\partial x_{i}}\right)+(1-\varpi) \frac{\partial}{\partial x_{k}}\left(v_{k}{ }^{n} \frac{\partial\left(\Delta \rho v_{i}\right)_{I}^{n+1}}{\partial x_{i}}\right)\right]\right\}
\end{aligned}
$$

en donde:

$$
\varpi=\left\{\begin{array}{ccc}
1 & \text { si } & M \leq 1 \\
-M+2 & \text { si } & 1<M<2 \\
0 & \text { si } & M \geq 2
\end{array}\right.
$$

Las ecuaciones de cantidad de movimiento, de energía y de transporte de $\widetilde{v}$ (SA) no se modifican con respecto al esquema TG2, y se mantienen los términos iterativos, por lo cual este esquema continúa respondiendo a la forma del esquema TG (no es CBS). Con respecto al esquema TG, únicamente se eliminan los términos donde aparecen las matrices $\left[A_{i j}^{P}\right],\left[A_{j}^{V}\right]$, $\left[A^{\bar{\varepsilon}}\right],\left[A_{i}^{\bar{\theta}}\right],\left[A^{\widetilde{v}}\right]$ y se modifica el térmico estabilizador en la ecuación de continuidad ponderado por la función $\varpi$ según se indica en (3.63) y (3.64).

Nuevamente, se procede a la discretización en el dominio espacial utilizando el método clásico de Bubnov-Galerkin. Por lo tanto, la única nueva expresión aquí es la ecuación de continuidad, ya que las otras ecuaciones sólo se modifican con respecto a las expresadas en (3.34) a (3.36) por la eliminación de los términos mencionados anteriormente. Los términos que involucran derivadas segundas de la posición en las expresiones aproximadas se integran por partes utilizando el teorema de Gauss-Green para relajar las exigencias sobre el orden de las derivadas de las funciones de interpolación. Se obtienen, así, las siguientes expresiones:

Ecuación de continuidad

$$
\begin{aligned}
\{\Delta \rho\}_{I+1}^{n+1}= & \Delta t_{\text {ext }}\left[M_{D}\right]^{-1}\left\{\varpi\left[\left(-\left[B_{i}\right]\left\{F_{i}^{M}\right\}^{n}-[P]\{p\}^{n}-\{h\}^{n}\right)+\frac{\Delta t_{\text {ext }}}{2}\left(-[P]\{\Delta p\}_{I}^{n+1}\right)\right]\right. \\
& \left.+(1-\varpi)\left[\left(-\left[B C_{i}\right]\left\{F_{i}^{M}\right\}^{n}\right)+\frac{\Delta t_{\text {ext }}}{2}\left(-\left[B C_{i}\right]\left\{\Delta F_{i}^{M}\right\}_{I}^{n+1}\right)\right]\right\}
\end{aligned}
$$

Ecuaciones de conservación de cantidad de movimiento

$$
\begin{aligned}
\left\{\Delta \rho v_{j}\right\}_{I+1}^{n+1} & =\Delta t_{\text {ext }}\left[M_{D}\right]^{-1}\left(-\left[B C_{i}\right]\left\{F_{i j}^{C M}\right\}^{n}-\left[D_{i j}\right]\left\{v_{i}\right\}^{n}+\left\{f_{j}\right\}^{n}\right) \\
& +\frac{\Delta t_{\text {ext }}}{2}\left[M_{D}\right]^{-1}\left(-\left[B C_{i}\right]\left\{\Delta F_{i j}^{C M}\right\}_{I}^{n+1}-\left[D_{i j}\right]\left\{\Delta v_{i}\right\}_{I}^{n+1}\right)
\end{aligned}
$$


Ecuaciones de conservación de energía

$$
\begin{aligned}
\{\Delta \rho e\}_{I+1}^{n+1}= & \Delta t_{\text {ext }}\left[M_{D}\right]^{-1}\left(-\left[B C_{i}\right]\left\{F_{i}^{E}\right\}^{n}-\left[E_{i}\right]\left\{v_{i}\right\}^{n}-[K]\{u\}^{n}+\{q\}^{n}\right) \\
& +\frac{\Delta t_{\text {ext }}}{2}\left[M_{D}\right]^{-1}\left(-\left[B C_{i}\right]\left\{\Delta F_{i}^{E}\right\}_{I}^{n+1}-\left[E_{i}\right]\left\{\Delta v_{i}\right\}_{I}^{n+1}-[K]\{\Delta u\}_{I}^{n+1}\right)
\end{aligned}
$$

Ecuación de transporte de $\tilde{v}(\mathrm{SA})$

$$
\begin{aligned}
\{\Delta \rho \tilde{v}\}_{I+1}^{n+1} & =\Delta t_{\text {ext }}\left[M_{D}\right]^{-1}\left(-\left[B C_{i}\right]\left\{F_{i}^{S A}\right\}^{n}-[M]\{Q\}^{n}+\left[L_{i}\right]\left\{Q v_{i}\right\}^{n}-\left[D_{v}\right]\{\tilde{v}\}^{n}\right) \\
& +\frac{\Delta t_{\text {ext }}}{2}\left[M_{D}\right]^{-1}\left(-\left[B C_{i}\right]\left\{\Delta F_{i}^{S A}\right\}_{I}^{n+1}-[M]\{\Delta Q\}_{I}^{n+1}-\left[D_{\tilde{v}}\right]\{\Delta \tilde{v}\}_{I}^{n+1}\right)
\end{aligned}
$$

Siendo todas las matrices y vectores los indicados en párrafos precedentes.

Es importante señalar que la función $\varpi ~(3.64)$ es una función local, que toma valores por nodo o elemento según la implementación. El número de Mach utilizado en su definición es el Mach local, calculado en los nodos o como un promedio de elemento. De esta forma, podrán existir regiones del dominio donde $\varpi$ adopte diferentes valores, impactando en consecuencia en las ecuaciones (3.63) y (3.65). Esta última variante, que integra en un único esquema las modificaciones dadas por las expresiones (3.60), (3.63) y (3.64) será denominada esquema MTG en este trabajo, por la abreviación de sus siglas en ingles "Modified Taylor Galerkin".

\subsection{MONTAJE, ACTUALIZACIÓN DE VARIABLES PRIMARIAS Y CÁLCULO DE VARIABLES SECUNDARIAS}

Todos los esquemas aquí planteados (TG, TG2, CBS, CBS single-step y MTG) plantean ecuaciones elementales para calcular los incrementos $\{\Delta \rho\}^{n+1},\left\{\Delta \rho v_{j}\right\}^{n+1},\{\Delta \rho e\}^{n+1}$ y $\{\Delta \rho \tilde{v}\}^{n+1}$ a partir de las variables conocidas en el paso de tiempo anterior. Pero estas ecuaciones de elemento aún deben ser ensambladas para formar un sistema global.

Una vez obtenido el sistema global este puede resolverse para obtener los incrementos para todos los nodos de la malla (a nivel global) y, entonces, deben actualizarse las variables primarias en toda la malla, es decir:

$$
\begin{gathered}
\{\rho\}^{n+1}=\{\rho\}^{n}+\{\Delta \rho\}^{n+1} \\
\left\{\rho v_{j}\right\}^{n+1}=\left\{\rho v_{j}\right\}^{n}+\left\{\Delta \rho v_{j}\right\}^{n+1} \\
\{\rho e\}^{n+1}=\{\rho e\}^{n}+\{\Delta \rho e\}^{n+1} \\
\{\rho \tilde{v}\}^{n+1}=\{\rho \tilde{v}\}^{n}+\{\Delta \rho \tilde{v}\}^{n+1}
\end{gathered}
$$

Luego, es posible obtener: 


$$
\begin{aligned}
\left\{v_{j}\right\}^{n+1} & =\left\{\frac{\rho v_{j}}{\rho}\right\}^{n+1} \\
\{e\}^{n+1} & =\left\{\frac{\rho e}{\rho}\right\}^{n+1} \\
\{\tilde{v}\}^{n+1} & =\left\{\frac{\rho \tilde{v}}{\rho}\right\}^{n+1}
\end{aligned}
$$

$\mathrm{y}$, finalmente, se utilizan las ecuaciones complementarias para obtener las variables secundarias, de la siguiente forma:

$$
\begin{gathered}
\{u\}^{n+1}=\left\{e-\frac{1}{2} v_{i} v_{i}-k_{t}\right\}^{n+1} \\
\{p\}^{n+1}=(\gamma-1)\{\rho u\}^{n+1}
\end{gathered}
$$

aunque aquí $k_{t}$ (energía cinética específica turbulenta) se desprecia. La viscosidad molecular y la conductividad térmica se calculan también nodo a nodo de la siguiente forma:

$$
\{\mu\}^{n+1}=\frac{M_{\infty}}{R_{e_{\infty}}}\left\{\frac{S+u_{\infty}}{S+u}\left(\frac{u}{u_{\infty}}\right)^{\frac{3}{2}}\right\}^{n+1} \quad ; \quad\{K\}^{n+1}=\frac{M_{\infty}}{R_{e_{\infty}}} \frac{\gamma}{\operatorname{Pr}_{\infty}}\left\{\frac{S_{k}+u_{\infty}}{S_{k}+u}\left(\frac{u}{u_{\infty}}\right)^{\frac{3}{2}}\right\}^{n+1}
$$

pero para su utilización en las expresiones integrales dentro de las matrices de elemento se utilizan valores promedio de elemento. También se obtienen las variables turbulentas actualizadas para toda la malla que surgen de las expresiones (2.44), (2.45) y (2.46). 


\section{CAPÍTULO 4}

\section{INTEGRACIÓN ANALÍTICA DE MATRICES ELEMENTALES}

\subsection{ELEMENTO ISOPARAMETRICO DE OCHO NODOS}

En este trabajo se utiliza el elemento hexaédrico trilineal de ocho nodos, utilizando las funciones de interpolación clásicas para expandir las componentes de cantidad de movimiento, energía, $\tilde{v}$ y densidad.

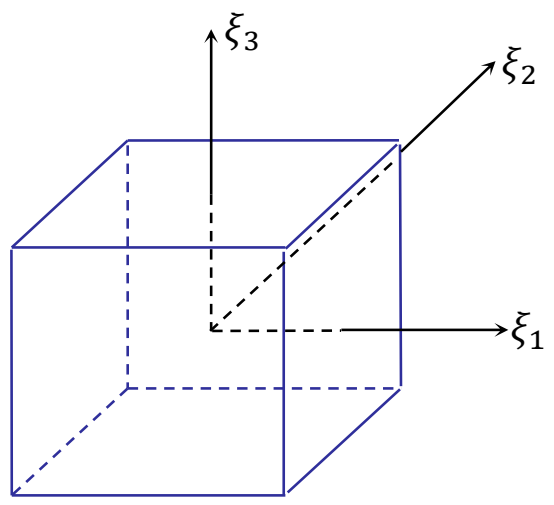

Figura 4.1-Espacio Computacional

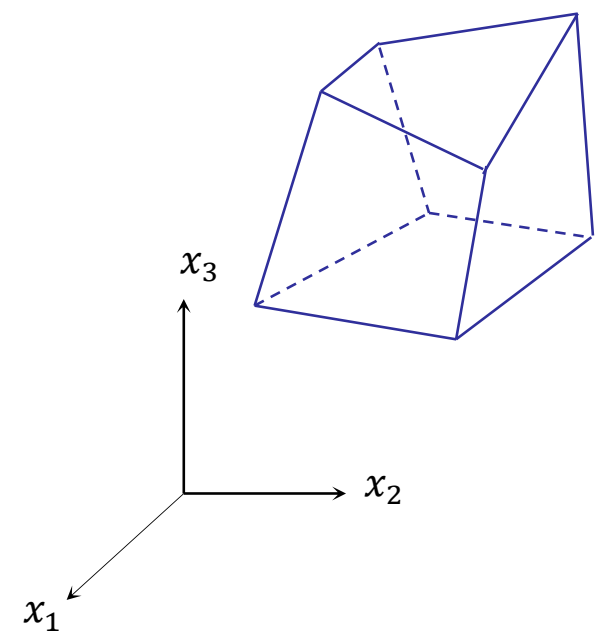

Figura 4.2-Espacio Físico

Las funciones de interpolación son las siguientes:

$$
\Phi_{N}=\frac{1}{8}\left[1+\xi_{1_{N}} \xi_{1}\right]\left[1+\xi_{2_{N}} \xi_{2}\right]\left[1+\xi_{3 N} \xi_{3}\right]
$$

donde el índice $N$ indica el número del nodo local, que varía de 1 a 8 . Por lo tanto, $\xi_{1_{N}} \xi_{2_{N}}$ y $\xi_{3_{N}}$ son las coordenadas naturales del nodo $N$ y son valores conocidos y fijos que se pueden agrupar en los siguientes arreglos:

$$
\begin{aligned}
& \left\{\xi_{1}\right\}^{T}=\left\{\begin{array}{rrrrrrrr}
-1 & 1 & 1 & -1 & -1 & 1 & 1 & -1
\end{array}\right\} \\
& \left\{\xi_{2}\right\}^{T}=\left\{\begin{array}{lrrrrrrr}
-1 & -1 & 1 & 1 & -1 & -1 & 1 & 1
\end{array}\right\} \\
& \left\{\xi_{3}\right\}^{T}=\left\{\begin{array}{llrrrrrr}
-1 & -1 & -1 & -1 & 1 & 1 & 1 & 1
\end{array}\right\}
\end{aligned}
$$


Cabe señalar que la expresión (4.1) está dada en notación de índices y no en forma matricial. Las funciones así obtenidas son las ocho funciones que componen la matriz línea descrita en la siguiente expresión vista anteriormente:

$$
[\Phi]=\left[\begin{array}{llllllll}
\Phi_{1} & \Phi_{2} & \Phi_{3} & \Phi_{4} & \Phi_{5} & \Phi_{6} & \Phi_{7} & \Phi_{8}
\end{array}\right]
$$

Como ya se ha mencionado anteriormente, todas las variables de campo se interpolan de la siguiente manera:

$$
f=[\Phi]\{f\}
$$

o también:

$$
f=\sum_{N=1}^{8} \Phi_{N} f_{N}
$$

Las expresiones (4.4) y (4.5) son equivalentes, la primera escrita en forma matricial y la segunda en notación de índices. Como se puede observar, para los subíndices nodales la forma adoptada aquí no sigue la convención de Einstein, que sí continúa siendo utilizada para subíndices que indican componentes de vectores y tensores en el espacio.

Dado que el elemento utilizado es isoparamétrico su geometría se puede interpolar de la misma forma, a partir de los valores nodales de coordenadas espaciales, de la siguiente manera:

$$
\begin{gathered}
x_{i}=[\Phi]\left\{x_{i}\right\} \\
x_{i}=\sum_{N=1}^{8} \Phi_{N} x_{i_{N}}
\end{gathered}
$$

\subsection{TRANSFORMACIÓN DEL DOMINIO DE INTEGRACIÓN}

Las matrices a nivel de elemento, ya definidas, vienen dadas en términos de funciones de interpolación y sus derivadas. Es decir, genéricamente, estas matrices son de la forma:

$$
\int_{\Omega_{E}} F\left([\Phi], \frac{\partial[\Phi]}{\partial x_{i}}\right) d \Omega
$$

Pero, el dominio de integración se transformará del espacio físico al espacio computacional, transformando la expresión genérica (4.8) en la siguiente expresión (también genérica): 


$$
\int_{-1}^{1} \int_{-1}^{1} \int_{-1}^{1} \mathrm{I}\left(\xi_{1}, \xi_{2}, \xi_{3}\right) d \xi_{1} d \xi_{2} d \xi_{3}
$$

Para ello, las derivadas de las funciones de interpolación con respecto a las coordenadas espaciales deben expresarse en términos de derivadas con respecto a las coordenadas naturales. Se sabe, de la expresión (4.1), que:

$$
\Phi_{N}=\Phi_{N}\left(\xi_{1}, \xi_{2}, \xi_{3}\right)
$$

y, de la expresión (4.7), se desprende que:

$$
x_{i}=x_{i}\left(\xi_{1}, \xi_{2}, \xi_{3}\right)
$$

Por lo tanto, considerando la regla de la cadena, se tiene:

$$
\frac{\partial \Phi_{N}}{\partial \xi_{j}}=\frac{\partial \Phi_{N}}{\partial x_{i}} \frac{\partial x_{i}}{\partial \xi_{j}}
$$

donde $i, j=1,2,3$ y $N=1,2, \ldots, 8$. En forma matricial, para las funciones de interpolación resulta la siguiente expresión:

$$
\frac{\partial[\Phi]}{\partial \xi_{j}}=\frac{\partial[\Phi]}{\partial x_{i}} \frac{\partial x_{i}}{\partial \xi_{j}}
$$

Desarrollando para los subíndices espaciales $i$ y $j$, según la convención de suma de Einstein, se puede expresar:

$$
\left\{\begin{array}{c}
\frac{\partial[\Phi]}{\partial \xi_{1}} \\
\frac{\partial[\Phi]}{\partial \xi_{2}} \\
\frac{\partial[\Phi]}{\partial \xi_{3}}
\end{array}\right\}=\left[\begin{array}{lll}
\frac{\partial x_{1}}{\partial \xi_{1}} & \frac{\partial x_{2}}{\partial \xi_{1}} & \frac{\partial x_{3}}{\partial \xi_{1}} \\
\frac{\partial x_{1}}{\partial \xi_{2}} & \frac{\partial x_{2}}{\partial \xi_{2}} & \frac{\partial x_{3}}{\partial \xi_{2}} \\
\frac{\partial x_{1}}{\partial \xi_{3}} & \frac{\partial x_{2}}{\partial \xi_{3}} & \frac{\partial x_{3}}{\partial \xi_{3}}
\end{array}\right]\left\{\begin{array}{c}
\frac{\partial[\Phi]}{\partial x_{1}} \\
\frac{\partial[\Phi]}{\partial x_{2}} \\
\frac{\partial[\Phi]}{\partial x_{3}}
\end{array}\right\}
$$

donde la matriz que contiene las derivadas $\frac{\partial x_{i}}{\partial \xi_{j}}$ es la Matriz Jacobiana de Transformación:

$$
\boldsymbol{J}=\left[J_{i j}\right]=\left[\begin{array}{lll}
\frac{\partial x_{1}}{\partial \xi_{1}} & \frac{\partial x_{2}}{\partial \xi_{1}} & \frac{\partial x_{3}}{\partial \xi_{1}} \\
\frac{\partial x_{1}}{\partial \xi_{2}} & \frac{\partial x_{2}}{\partial \xi_{2}} & \frac{\partial x_{3}}{\partial \xi_{2}} \\
\frac{\partial x_{1}}{\partial \xi_{3}} & \frac{\partial x_{2}}{\partial \xi_{3}} & \frac{\partial x_{3}}{\partial \xi_{3}}
\end{array}\right]
$$

Cabe señalar que $J_{i j}=\frac{\partial x_{i}}{\partial \xi_{j}}=f\left(\xi_{1}, \xi_{2}, \xi_{3}\right)$ y, teniendo en cuenta las expresiones (4.6), se obtiene: 


$$
\boldsymbol{J}\left(\xi_{1}, \xi_{2}, \xi_{3}\right)=\left[\begin{array}{lll}
\frac{\partial[\Phi]}{\partial \xi_{1}}\left\{x_{1}\right\} & \frac{\partial[\Phi]}{\partial \xi_{1}}\left\{x_{2}\right\} & \frac{\partial[\Phi]}{\partial \xi_{1}}\left\{x_{3}\right\} \\
\frac{\partial[\Phi]}{\partial \xi_{2}}\left\{x_{1}\right\} & \frac{\partial[\Phi]}{\partial \xi_{2}}\left\{x_{2}\right\} & \frac{\partial[\Phi]}{\partial \xi_{2}}\left\{x_{3}\right\} \\
\frac{\partial[\Phi]}{\partial \xi_{3}}\left\{x_{1}\right\} & \frac{\partial[\Phi]}{\partial \xi_{3}}\left\{x_{2}\right\} & \frac{\partial[\Phi]}{\partial \xi_{3}}\left\{x_{3}\right\}
\end{array}\right]
$$

o también:

$$
J_{i j}=\frac{\partial[\Phi]}{\partial \xi_{i}}\left\{x_{j}\right\}
$$

Dado que se requiere expresar a las derivadas $\frac{\partial[\Phi]}{\partial x_{i}}$ según las derivadas $\frac{\partial[\Phi]}{\partial \xi_{j}}$, resulta necesario invertir el sistema de ecuaciones dado por (4.14), obteniendo:

$$
\frac{\partial[\Phi]}{\partial x_{j}}=J_{i j}^{-1} \frac{\partial[\Phi]}{\partial \xi_{i}}
$$

donde la inversa de la matriz jacobiana se puede obtener mediante la siguiente expresión:

$$
J^{-1}=\frac{\bar{J}}{|J|}
$$

siendo $\overline{\boldsymbol{J}}$ la transpuesta de la matriz adjunta de la matriz $\boldsymbol{J}$.

Por lo tanto, sólo queda por expresar el diferencial de volumen en términos de las coordenadas naturales, con el fin de transformar a las integrales de la forma (4.8) en integrales de la forma (4.9). Sabiendo que:

$$
d \Omega=|J| d \xi_{1} d \xi_{2} d \xi_{3}
$$

sólo resta substituir las expresiones (4.18) y la expresión (4.20) en (4.8) para obtener las integrales de la forma (4.9), las cuales deben integrarse, ya sea analíticamente o utilizando un método de integración numérica como, por ejemplo, el método Gauss-Legendre.

\subsection{INTEGRACIÓN ANALÍTICA DE MATRICES ELEMENTALES 1: J(0)}

Las integrales de tipo (4.9) se pueden resolver numéricamente mediante el método Gauss-Legendre. Sin embargo, el cómputo de estas integrales es un proceso oneroso desde el punto de vista del tiempo necesario para su ejecución. En 3D, con hexaedros lineales, se requieren 8 puntos de cuadratura de Gauss para el proceso de integración. Solamente este proceso de integración penaliza la velocidad de ejecución entre 7-8 veces cuando se lo compara con implementaciones de diferencias finitas o volúmenes finitos ${ }^{[26]}$. 
Pero, para reducir el tiempo computacional y el espacio de memoria requerida, algunos investigadores ${ }^{[24]}$ proponen utilizar un único punto de cuadratura para calcular las integrales. Una forma de hacer esto sería calcular los integrandos de las expresiones del tipo de la (4.9) en el centro del elemento, donde $\left(\xi_{1}=0, \xi_{2}=0, \xi_{3}=0\right)$ y con ello tener un valor constante para el elemento, lo cual permite evitar la integración (o equivaldría a integrar numéricamente con un único punto de Gauss). Basados en la idea de que el proceso descripto anteriormente conduciría a la aparición de oscilaciones espurias (Hourglassing), algunos investigadores proponen calcular los integrandos en los nodos del elemento y luego promediarlos y aplicarlos en el punto central ${ }^{[26]}$. Así, nuevamente se evita la integración.

Sin embargo, las dos estrategias anteriores acarrean pérdida de exactitud, ya que implican tomar valores constantes de elemento dentro de las integrales. Es por ello por lo que algunos autores ${ }^{[49]}$ proponen integrar analíticamente las expresiones del tipo de las (4.9) para elementos cuadrangulares isoparamétricos bilineales. Otros investigadores ${ }^{[25]}$ extendieron esta idea para el caso de hexaedros en 3D utilizando el punto central para definir al Jacobiano y su determinante, pero manteniendo el resto de los factores tal cual son, para luego integrar analíticamente y utilizar directamente en el código las expresiones explícitas que resultan. De esta manera las expresiones obtenidas son más exactas que las obtenidas con un punto de cuadratura. Las fórmulas así calculadas serán exactas en caso de una malla de hexaedros de caras paralelas y dará lugar a una buena aproximación cuando los hexaedros estén distorsionados ${ }^{[25]}$.

En este trabajo se adoptan dos estrategias de integración diferentes. La primera es, precisamente, la descripta en los párrafos anteriores, es decir, utilizando un punto de integración en el centro del elemento $\left(\xi_{1}=0, \xi_{2}=0, \xi_{3}=0\right)$ sólo para calcular la matriz Jacobiana y su determinante. El resto de los factores del integrando permanecen como están y se realiza la integración analítica. La segunda estrategia consiste en calcular las matrices de masa $[M]$ y $\left[M_{D}\right]$ y matrices convectivas $\left[B_{i}\right]$ en forma exacta, sin tomar los jacobianos en el centro del elemento sino considerando la dependencia del Jacobiano con la posición, y sólo las matrices difusivas $\left[D_{i j}\right],\left[E_{i}\right]$ y $[K]$ y las matrices de estabilización, como por ejemplo la matriz $\left[C_{i}\right]$, se calculan tomando los jacobianos en el centro del elemento. Esta segunda estrategia de integración se explica en la sección 4.4.

Para desarrollar la primera estrategia de integración utilizada en este trabajo, considerando la expresión (4.17) y calculando las derivadas $\frac{\partial[\Phi]}{\partial \xi_{i}}$ en el centro del elemento, se obtiene la siguiente matriz Jacobiana: 


$$
J(0)=\left[\begin{array}{lll}
J_{11}(0) & J_{12}(0) & J_{13}(0) \\
J_{21}(0) & J_{22}(0) & J_{23}(0) \\
J_{31}(0) & J_{32}(0) & J_{33}(0)
\end{array}\right]=\frac{1}{8}\left[\begin{array}{lll}
\left\{\xi_{1}\right\}^{T}\left\{x_{1}\right\} & \left\{\xi_{1}\right\}^{T}\left\{x_{2}\right\} & \left\{\xi_{1}\right\}^{T}\left\{x_{3}\right\} \\
\left\{\xi_{2}\right\}^{T}\left\{x_{1}\right\} & \left\{\xi_{2}\right\}^{T}\left\{x_{2}\right\} & \left\{\xi_{2}\right\}^{T}\left\{x_{3}\right\} \\
\left\{\xi_{3}\right\}^{T}\left\{x_{1}\right\} & \left\{\xi_{3}\right\}^{T}\left\{x_{2}\right\} & \left\{\xi_{3}\right\}^{T}\left\{x_{3}\right\}
\end{array}\right]
$$

y su inversa:

$$
\boldsymbol{J}^{-1}(0)=\frac{\overline{\boldsymbol{J}(0)}}{|\boldsymbol{J}(0)|}
$$

donde la traspuesta de la matriz adjunta es:

$$
\overline{\boldsymbol{J}(0)}=\left[\begin{array}{lll}
{\left[J_{22} J_{33}-J_{23} J_{32}\right]_{(0)}} & {\left[J_{13} J_{32}-J_{12} J_{33}\right]_{(0)}} & {\left[J_{12} J_{23}-J_{13} J_{22}\right]_{(0)}} \\
{\left[J_{23} J_{31}-J_{21} J_{33}\right]_{(0)}} & {\left[J_{11} J_{33}-J_{13} J_{31}\right]_{(0)}} & {\left[J_{31} J_{21}-J_{11} J_{23}\right]_{(0)}} \\
{\left[J_{21} J_{32}-J_{22} J_{31}\right]_{(0)}} & {\left[J_{12} J_{31}-J_{11} J_{32}\right]_{(0)}} & {\left[J_{11} J_{22}-J_{12} J_{21}\right]_{(0)}}
\end{array}\right]
$$

Así, se tiene todo lo necesario para integrar analíticamente las matrices elementales expresadas en el capítulo anterior.

\subsubsection{Matrices $[M]$ :}

$$
M_{M N}=\int_{\Omega_{E}} \Phi_{M} \Phi_{N} d \Omega=\int_{-1}^{1} \int_{-1}^{1} \int_{-1}^{1} \Phi_{M} \Phi_{N}|J(0)| d \xi_{1} d \xi_{2} d \xi_{3}
$$

donde $M, N=1,2, \ldots, 8 \mathrm{y}$, luego de un proceso de integración que es bastante obvio, para esta matriz se obtiene lo siguiente:

$$
M_{M N}=\frac{\Omega_{E}}{64}\left[1+\frac{1}{3} \xi_{1_{M}} \xi_{1_{N}}\right]\left[1+\frac{1}{3} \xi_{2 M} \xi_{2 N}\right]\left[1+\frac{1}{3} \xi_{3 M} \xi_{3 N}\right]
$$

Si la matriz anterior se diagonaliza, se obtiene:

$$
M_{D_{M N}}=\frac{\Omega_{E}}{8} \delta_{M N}
$$

4.3.2 Matrices $\left[\alpha_{i j}\right]$ :

$$
\begin{aligned}
\alpha_{i j_{M N}}=\frac{\Delta t_{i n}}{2} & \int_{\Omega_{E}} \frac{\partial \Phi_{M}}{\partial x_{i}} \frac{\partial \Phi_{N}}{\partial x_{j}} d \Omega \\
& =\frac{\Delta t_{i n}}{2} \int_{-1}^{1} \int_{-1}^{1} \int_{-1}^{1}\left(\frac{\partial \Phi_{M}}{\partial \xi_{k}} J(0)_{i k}^{-1}\right)\left(\frac{\partial \Phi_{N}}{\partial \xi_{h}} J(0)_{j h}^{-1}\right)|J(0)| d \xi_{1} d \xi_{2} d \xi_{3}
\end{aligned}
$$

donde $i, j, k, h=1,2,3$ y $M, N=1,2, \ldots, 8$. Estas matrices resultan ser simétricas.

Dado que la obtención de expresiones analíticas para las matrices $\left[\alpha_{i j}\right]$ no es tan obvia como en los casos anteriores, aquí se presenta, a modo de ejemplo, el proceso completo de 
integración de estas matrices. Recordando que $\overline{\boldsymbol{J}(0)}$ y $|\boldsymbol{J}(0)|$ son constantes en el elemento, la expresión (4.27) se puede transformar de la siguiente manera:

$$
\alpha_{i j_{M N}}=\frac{\Delta t_{i n}}{2} \frac{\overline{J(0)_{k i}} \overline{J(0)_{j h}}}{|J(0)|} \int_{-1}^{1} \int_{-1}^{1} \int_{-1}^{1} \frac{\partial \Phi_{M}}{\partial \xi_{k}} \frac{\partial \Phi_{N}}{\partial \xi_{h}} d \xi_{1} d \xi_{2} d \xi_{3}
$$

A continuación, derivando en (4.1), las derivadas que aparecen en (4.28) se pueden expresar de la siguiente manera:

$$
\frac{\partial \Phi_{M}}{\partial \xi_{k}}=\frac{1}{8} \xi_{k_{M}}\left[1+\xi_{a_{M}} \xi_{(a)}\right]\left[1+\xi_{b_{M}} \xi_{(b)}\right]
$$

en donde el paréntesis en los subíndices indica que la convención de suma no se aplica, aún si estando repetidos los índices. En la expresión (4.29) se tiene que:

$$
k=1,2,3, y\left\{\begin{array}{llll}
\text { si } k=1 & \rightarrow & a=2 & b=3 \\
\text { si } k=2 & \rightarrow & a=1 & b=3 \\
\text { si } k=3 & \rightarrow & a=1 & b=2
\end{array}\right.
$$

A continuación, reemplazando (4.29) en (4.28) da como resultado:

$$
\begin{aligned}
\alpha_{i j_{M N}}=\frac{\Delta t_{i n}}{2} & \frac{\overline{J(0)_{k i}} \overline{J(0)_{j h}}}{64|J(0)|} \int_{-1}^{1} \int_{-1}^{1} \int_{-1}^{1} \xi_{k_{M}} \xi_{h_{N}}\left[1+\xi_{a_{M}} \xi_{(a)}\right]\left[1+\xi_{b_{M}} \xi_{(b)}\right][1 \\
& \left.+\xi_{c_{N}} \xi_{(c)}\right]\left[1+\xi_{d_{N}} \xi_{(d)}\right] d \xi_{1} d \xi_{2} d \xi_{3}
\end{aligned}
$$

siendo:

$$
h=1,2,3, y\left\{\begin{array}{llll}
\text { si } h=1 & \rightarrow & c=2 & d=3 \\
\text { si } h=2 & \rightarrow & c=1 & d=3 \\
\text { si } h=3 & \rightarrow & c=1 & d=2
\end{array}\right.
$$

Adoptando la siguiente denominación:

$$
\begin{aligned}
I_{k h_{M N}}=\xi_{k_{M}} & \xi_{h_{N}} \int_{-1}^{1} \int_{-1}^{1} \int_{-1}^{1}\left[1+\xi_{a_{M}} \xi_{(a)}\right]\left[1+\xi_{b_{M}} \xi_{(b)}\right]\left[1+\xi_{c_{N}} \xi_{(c)}\right][1 \\
& \left.+\xi_{d_{N}} \xi_{(d)}\right] d \xi_{1} d \xi_{2} d \xi_{3}
\end{aligned}
$$

$\mathrm{y}$, recordando que:

$$
\Omega_{E}=8|J(0)|
$$

la (4.31) resulta:

$$
\alpha_{i j_{M N}}=\frac{\Delta t_{i n}}{2} \frac{\overline{J(0)_{k i}} \overline{J(0)_{j h}}}{8 \Omega_{E}} I_{k h_{M N}}
$$


La integral en (4.35) se puede calcular para un caso determinado y luego deducir una expresión genérica a partir del caso particular. Por ejemplo, si $k=1$ y $h=2$ se tiene:

$$
\begin{aligned}
& \left\{\begin{array}{llll}
k=1 & \rightarrow & a=2 & b=3 \\
h=2 & \rightarrow & c=1 & d=3
\end{array}\right. \\
& I_{12 M N}=\xi_{1{ }_{M}} \xi_{2 N} \int_{-1}^{1} \int_{-1}^{1} \int_{-1}^{1}\left[1+\xi_{2} \xi_{2}\right]\left[1+\xi_{3 M} \xi_{3}\right]\left[1+\xi_{1_{N}} \xi_{1}\right][1 \\
& \left.+\xi_{3 N} \xi_{3}\right] d \xi_{1} d \xi_{2} d \xi_{3}
\end{aligned}
$$

Así, integrando una vez, resulta:

$$
\begin{aligned}
I_{12 M N}=\left[\xi_{1}+\right. & \left.\xi_{1_{N}} \frac{\xi_{1}^{2}}{2}\right]_{-1}^{1} \xi_{1_{M}} \xi_{2 N} \int_{-1}^{1} \int_{-1}^{1}\left[1+\xi_{2_{M}} \xi_{2}\right]\left[1+\xi_{3_{M}} \xi_{3}\right][1 \\
& \left.+\xi_{3_{N}} \xi_{3}\right] d \xi_{2} d \xi_{3}
\end{aligned}
$$

Sin embargo, el factor ya integrado entre corchetes puede calcularse y se observa que es igual a dos. En efecto:

$$
\left[\xi_{1}+\xi_{1_{N}} \frac{\xi_{1}^{2}}{2}\right]_{-1}^{1}=\left[1+\xi_{1_{N}} \frac{1^{2}}{2}\right]-\left[(-1)+\xi_{1_{N}} \frac{(-1)^{2}}{2}\right]=2
$$

$\mathrm{y}$, entonces:

$$
\begin{aligned}
I_{12 M N} & =2 \xi_{1_{M}} \xi_{2_{N}} \int_{-1}^{1} \int_{-1}^{1}\left[1+\xi_{2 M} \xi_{2}\right]\left[1+\xi_{3_{M}} \xi_{3}\right]\left[1+\xi_{3_{N}} \xi_{3}\right] d \xi_{2} d \xi_{3} \\
& =2\left[\xi_{2}+\xi_{2_{M}} \frac{\xi_{2}^{2}}{2}\right]_{-1}^{1} \xi_{1_{M}} \xi_{2_{N}} \int_{-1}^{1}\left[1+\xi_{3_{M}} \xi_{3}\right]\left[1+\xi_{3_{N}} \xi_{3}\right] d \xi_{3} \\
& =4 \xi_{1_{M}} \xi_{2_{N}} \int_{-1}^{1}\left[1+\xi_{3_{M}} \xi_{3}+\xi_{3_{N}} \xi_{3}+\xi_{3_{M}} \xi_{3_{N}} \xi_{3}^{2}\right] d \xi_{3} \\
& =4\left[\xi_{3}+\xi_{3_{M}} \frac{\xi_{3}^{2}}{2}+\xi_{3_{N}} \frac{\xi_{3}^{2}}{2}+\xi_{3_{M}} \xi_{3_{N}} \frac{\xi_{3}{ }^{3}}{2}\right]_{-1}^{1} \xi_{1_{M}} \xi_{2_{N}}
\end{aligned}
$$

donde para el factor entre corchetes se tiene:

$$
\begin{gathered}
{\left[\xi_{3}+\xi_{3_{M}} \frac{\xi_{3}^{2}}{2}+\xi_{3_{N}} \frac{\xi_{3}^{2}}{2}+\xi_{3_{M}} \xi_{3_{N}} \frac{\xi_{3}^{3}}{2}\right]_{-1}^{1}=\left[1+\xi_{3_{M}} \frac{1^{2}}{2}+\xi_{3_{N}} \frac{1^{2}}{2}+\xi_{3_{M}} \xi_{3_{N}} \frac{1^{3}}{2}\right]} \\
-\left[(-1)+\xi_{3_{M}} \frac{(-1)^{2}}{2}+\xi_{3_{N}} \frac{(-1)^{2}}{2}+\xi_{3_{M}} \xi_{3_{N}} \frac{(-1)^{3}}{2}\right]=2\left[1+\frac{1}{3} \xi_{3_{M}} \xi_{3_{N}}\right]
\end{gathered}
$$

El reemplazo de estas últimas expresiones en (4.36) finalmente resulta: 


$$
I_{12 M N}=8 \xi_{1_{M}} \xi_{2 N}\left[1+\frac{1}{3} \xi_{3 M} \xi_{3_{N}}\right]
$$

La expresión (4.37) indica el valor de la integral $I_{k h_{M N}}$ para el caso en el que $k=1$ y $h=2$. Sin embargo, a partir de (4.37), se puede deducir el valor de la integral para el caso genérico. De hecho, se observa que:

$$
\text { si } k \neq h \quad \rightarrow \quad \mathrm{I}_{k h_{M N}}=8 \xi_{k_{M}} \xi_{h_{N}}\left[1+\frac{1}{3} \xi_{(s)} \xi_{s_{N}}\right]
$$

donde:

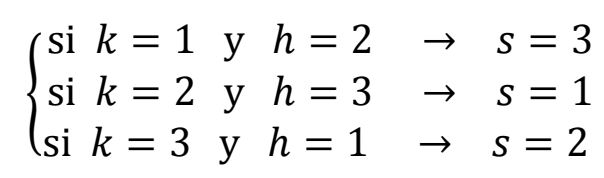

A su vez, si $k=h$, a partir de (4.31) y (4.33) se tienen las siguientes igualdades:

$$
\begin{aligned}
& a=c \\
& b=d
\end{aligned}
$$

motivo por el cual, una vez que se introducen estas igualdades en (4.33), la integración de esta última conduce a la siguiente expresión:

$$
\text { si } k=h \rightarrow I_{k h_{M N}}=8 \xi_{k_{M}} \xi_{h_{N}}\left[1+\frac{1}{3} \xi_{(s)_{M}} \xi_{s_{N}}\right]\left[1+\frac{1}{3} \xi_{(r)_{M}} \xi_{r_{N}}\right]
$$

dónde:

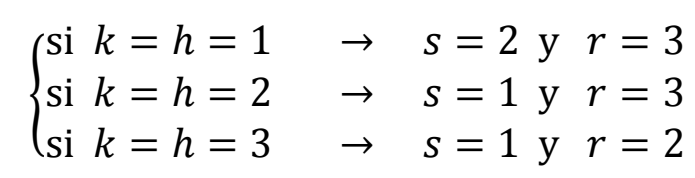

Por lo tanto, denominando:

$$
\pi_{k h_{M N}}=\frac{I_{k h_{M N}}}{8}
$$

la expresión (4.27) resulta, finalmente:

$$
\alpha_{i j_{M N}}=\frac{\Delta t_{i n}}{2} \frac{1}{\Omega_{E}} \mathcal{A}_{i j_{M N}}
$$

donde:

$$
\mathcal{A}_{i j_{M N}}=\overline{J_{k i}(0)} \overline{J_{j h}(0)} \pi_{k h_{M N}}
$$

con el factor $\pi_{k h_{M N}}$ definido de la siguiente forma: 


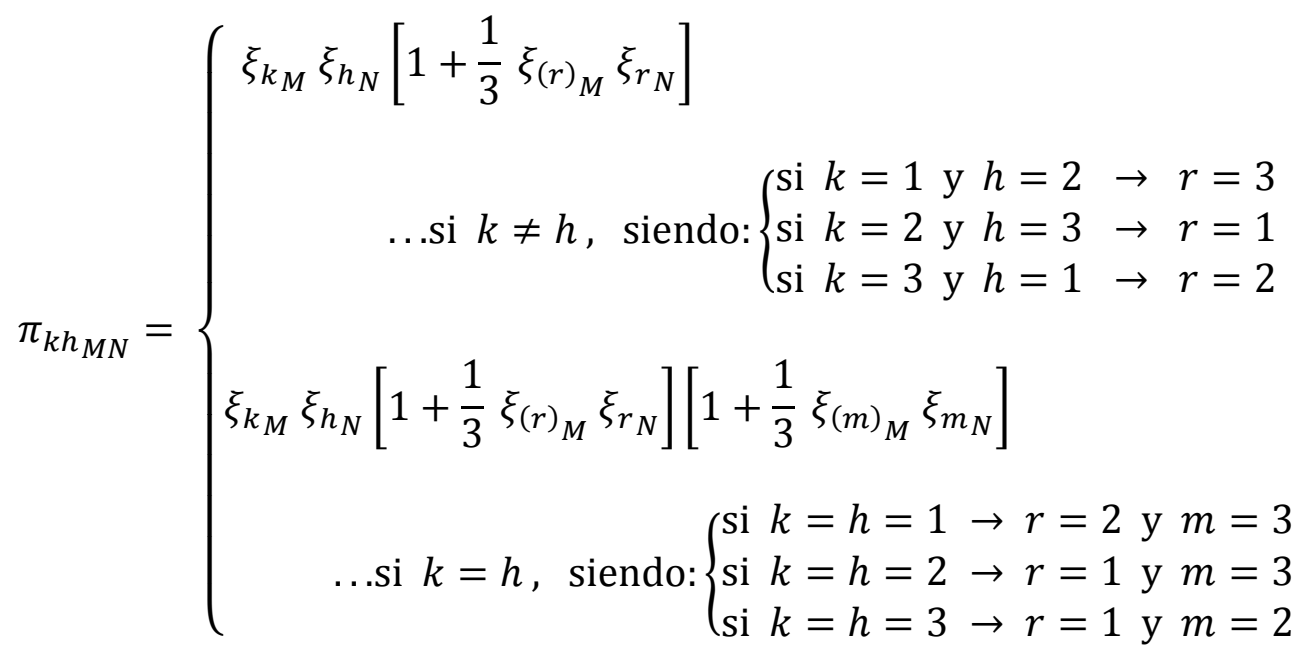

donde el paréntesis en el subíndice indica que no se aplica la convención de suma, aún con los índices repetidos.

En forma análoga al proceso de integración de las matrices $\left[\alpha_{i j}\right]$, otras matrices se pueden integrar rápidamente teniendo en cuenta su similitud con ellas. Este es el caso de las matrices $\left[C_{i}\right],\left[A_{i j}^{P}\right],\left[A_{j}^{V}\right],\left[A^{\bar{\varepsilon}}\right],\left[A_{i}^{\bar{\theta}}\right]$ y $\left[A^{\widetilde{v}}\right],\left[D_{i j}\right],[K]$ y $\left[B_{\mathrm{i}}\right]$ que contienen integrales similares a la de la expresión (4.29). En el caso de las matrices $\left[D_{i j}\right]$ y $[K]$, los coeficientes $\eta$, $\lambda$ y $\kappa$ son funciones de la posición. Para simplificar su integración, los valores de estos coeficientes se calculan en los nodos y, a continuación, se promedian los valores nodales para obtener un valor de elemento. Por lo tanto, los coeficientes $\eta, \lambda$ y $\kappa$ se consideran constantes en el elemento y pueden salir de las integrales respectivas.

\subsubsection{Matrices $\left[C_{i}\right],\left[A_{i j}^{P}\right],\left[A_{j}^{V}\right],\left[A^{\bar{\varepsilon}}\right],\left[A_{i}^{\bar{\theta}}\right] y\left[A^{\widetilde{v}}\right]$ :}

Estas matrices tienen la forma de las matrices $\left[\alpha_{i j}\right]$ pero están premultiplicadas por valores de velocidad, presión u otras variables o productos de variables, que en algunos casos suman con los índices para dar diferentes expresiones.

Por ejemplo, la matriz $\left[C_{i}\right]$ es:

$$
\begin{aligned}
C_{i_{M N}} & =\frac{\Delta t_{i n}}{2} \int_{\Omega_{E}}\left(\sum_{L=1}^{8} \Phi_{L} v_{j}^{n}\right) \frac{\partial \Phi_{M}}{\partial x_{j}} \frac{\partial \Phi_{N}}{\partial x_{i}} d \Omega \\
& =\left(v_{j}^{n}\right)_{E} \frac{\Delta t_{i n}}{2} \int_{-1}^{1} \int_{-1}^{1} \int_{-1}^{1}\left(\frac{\partial \Phi_{M}}{\partial \xi_{k}} J(0)_{j k}^{-1}\right)\left(\frac{\partial \Phi_{N}}{\partial \xi_{h}} J(0)_{i h}^{-1}\right)|J(0)| d \xi_{1} d \xi_{2} d \xi_{3}
\end{aligned}
$$

donde $i, j, k, h=1,2,3$ y $M, N=1,2, \ldots, 8$. 
En estas últimas expresiones, el factor entre paréntesis $\left(\sum_{L=1}^{8} \Phi_{L} v_{k_{L}}^{n}\right)$ ha sido simplificado tomando funciones de interpolación en el centro del elemento. Esto equivale a tomar el promedio de los valores nodales de la variable que, debido a que es un valor definido para todo el elemento, puede salir de la integral simplificando la integración. El resultado es una velocidad promedio de elemento:

$$
\left(v_{j}^{n}\right)_{E}=\frac{1}{8} \sum_{M=1}^{8} v_{j}^{n}
$$

La pérdida de precisión será menor cuanto menor sea el tamaño del elemento. Con esta simplificación adicional, resulta obvio que:

$$
C_{i_{M N}}=\left(v_{j}^{n}\right)_{E} \alpha_{j i}=\left(v_{j}^{n}\right)_{E} \frac{\Delta t_{i n}}{2} \frac{1}{\Omega_{E}} \mathcal{A}_{j i}{ }_{M N}
$$

De la misma forma se tiene:

$$
\begin{gathered}
A_{i j_{M N}}^{P}=\left(p^{n}\right)_{E} \alpha_{j i_{M N}}=\left(p^{n}\right)_{E} \frac{\Delta t_{i n}}{2} \frac{1}{\Omega_{E}} \mathcal{A}_{j i_{M N}} \\
A_{j_{M N}^{V}}^{V}=\left(v_{j}^{n}\right)_{E} \alpha_{i i_{M N}}=\left(v_{j}^{n}\right)_{E} \frac{\Delta t_{i n}}{2} \frac{1}{\Omega_{E}} \mathcal{A}_{i i_{M N}} \\
A_{M N}^{\bar{\varepsilon}}=\left(\bar{\varepsilon}^{n}\right)_{E} \alpha_{i i_{M N}}=\left(\bar{\varepsilon}^{n}\right)_{E} \frac{\Delta t_{i n}}{2} \frac{1}{\Omega_{E}} \mathcal{A}_{i i_{M N}} \\
\bar{\varepsilon}=\gamma e-\bar{\theta} ; \quad \bar{\theta}=(\gamma-1) \frac{|\boldsymbol{V}|^{2}}{2} \\
A_{i_{M N}}^{\bar{\theta}}=\left(\bar{\theta}^{n} v_{j}^{n}\right)_{E} \alpha_{j i_{M N}}=\left(\bar{\theta}^{n} v_{j}^{n}\right)_{E} \frac{\Delta t_{i n}}{2} \frac{1}{\Omega_{E}} \mathcal{A}_{j i}{ }_{M N} \\
A_{M N}^{\widetilde{v}}=\left(\tilde{v}^{n}\right)_{E} \alpha_{i i_{M N}}=\left(\tilde{v}^{n}\right)_{E} \frac{\Delta t_{i n}}{2} \frac{1}{\Omega_{E}} \mathcal{A}_{i i_{M N}}
\end{gathered}
$$

\subsubsection{Matrices $\left[B_{i}\right]$ :}

$$
B_{i_{M N}}=\int_{\Omega_{E}} \Phi_{M} \frac{\partial \Phi_{N}}{\partial x_{i}} d \Omega=\int_{-1}^{1} \int_{-1}^{1} \int_{-1}^{1} \Phi_{M} \frac{\partial \Phi_{N}}{\partial \xi_{j}} J(0)_{i j}^{-1}|J(0)| d \xi_{1} d \xi_{2} d \xi_{3}
$$

Integrando, resulta: 


$$
\begin{aligned}
B_{i_{M N}}= & \frac{1}{8}\left\{\overline{J_{i 1}(0)} \xi_{1_{N}}\left[1+\frac{1}{3} \xi_{2_{M}} \xi_{2_{N}}\right]\left[1+\frac{1}{3} \xi_{3_{M}} \xi_{3_{N}}\right]\right. \\
& +\overline{J_{i 2}(0)} \xi_{2_{N}}\left[1+\frac{1}{3} \xi_{1_{M}} \xi_{1_{N}}\right]\left[1+\frac{1}{3} \xi_{3_{M}} \xi_{3_{N}}\right] \\
& \left.+\overline{J_{i 3}(0)} \xi_{3_{N}}\left[1+\frac{1}{3} \xi_{1_{M}} \xi_{1_{N}}\right]\left[1+\frac{1}{3} \xi_{2_{M}} \xi_{2_{N}}\right]\right\}
\end{aligned}
$$

\subsubsection{Matrices $\left[D_{i j}\right]$}

En caso de que $i \neq j$, se tiene:

$$
\begin{aligned}
D_{i j_{M N}}= & \int_{\Omega_{E}} \eta \frac{\partial \Phi_{M}}{\partial x_{i}} \frac{\partial \Phi_{N}}{\partial x_{j}} d \Omega+\int_{\Omega_{E}} \lambda \frac{\partial \Phi_{M}}{\partial x_{j}} \frac{\partial \Phi_{N}}{\partial x_{i}} d \Omega \\
& =\int_{-1}^{1} \int_{-1}^{1} \int_{-1}^{1} \eta\left(\frac{\partial \Phi_{M}}{\partial \xi_{k}} J(0)_{i k}^{-1}\right)\left(\frac{\partial \Phi_{N}}{\partial \xi_{h}} J(0)_{j h}^{-1}\right)|J(0)| d \xi_{1} d \xi_{2} d \xi_{3} \\
& +\int_{-1}^{1} \int_{-1}^{1} \int_{-1}^{1} \lambda\left(\frac{\partial \Phi_{M}}{\partial \xi_{k}} J(0)_{j k}^{-1}\right)\left(\frac{\partial \Phi_{N}}{\partial \xi_{h}} J(0)_{i h}^{-1}\right)|J(0)| d \xi_{1} d \xi_{2} d \xi_{3}
\end{aligned}
$$

Cabe señalar que $D_{i j_{M N}}=D_{j i}{ }_{N M}$ o, en notación matricial $\left[D_{i j}\right]=\left[D_{i j}\right]^{T}$. A continuación, integrando la expresión (4.56) se obtiene:

$$
D_{i j_{M N}}=(\eta)_{E} \frac{1}{\Omega_{E}} \mathcal{A}_{i j_{M N}}+(\lambda)_{E} \frac{1}{\Omega_{E}} \mathcal{A}_{j i}{ }_{M N}
$$

Por otro lado, en el caso de ser $i=j$, considerando que:

$$
\begin{aligned}
& \text { si } i=1 \rightarrow k=2,3 \\
& \text { si } i=2 \rightarrow k=1,3 \\
& \text { si } i=3 \rightarrow k=1,2
\end{aligned}
$$

entonces, para $l, h=1,2,3$, se obtiene la siguiente expresión:

$$
\begin{aligned}
D_{i(i)_{M N}}= & \int_{\Omega_{E}}(2 \eta+\lambda) \frac{\partial \Phi_{M}}{\partial x_{i}} \frac{\partial \Phi_{N}}{\partial x_{(i)}} d \Omega+\int_{\Omega_{E}} \eta \frac{\partial \Phi_{M}}{\partial x_{k}} \frac{\partial \Phi_{N}}{\partial x_{k}} d \Omega \\
& =\int_{-1}^{1} \int_{-1}^{1} \int_{-1}^{1}(2 \eta+\lambda)\left(\frac{\partial \Phi_{M}}{\partial \xi_{l}} J(0)_{i l}^{-1}\right)\left(\frac{\partial \Phi_{N}}{\partial \xi_{h}} J(0)_{(i) h}^{-1}\right)|J(0)| d \xi_{1} d \xi_{2} d \xi_{3} \\
& +\int_{-1}^{1} \int_{-1}^{1} \int_{-1}^{1} \eta\left(\frac{\partial \Phi_{M}}{\partial \xi_{l}} J(0)_{k l}^{-1}\right)\left(\frac{\partial \Phi_{N}}{\partial \xi_{h}} J(0)_{k h}^{-1}\right)|J(0)| d \xi_{1} d \xi_{2} d \xi_{3}
\end{aligned}
$$

donde, nuevamente, los subíndices entre paréntesis no siguen la convención de suma. Así que integrando la expresión (4.59): 


$$
D_{i(i)_{M N}}=(2 \eta+\lambda)_{E} \frac{1}{\Omega_{E}} \mathcal{A}_{i(i)_{M N}}+(\eta)_{E} \frac{1}{\Omega_{E}} \mathcal{A}_{k k_{M N}}
$$

4.3.6 Matriz $[K]$ :

$$
\begin{aligned}
K_{M N}= & \int_{\Omega_{E}} \kappa \frac{\partial \Phi_{M}}{\partial x_{i}} \frac{\partial \Phi_{N}}{\partial x_{i}} d \Omega \\
& =\int_{-1}^{1} \int_{-1}^{1} \int_{-1}^{1} \kappa\left(\frac{\partial \Phi_{M}}{\partial \xi_{j}} J(0)_{i j}^{-1}\right)\left(\frac{\partial \Phi_{N}}{\partial \xi_{h}} J(0)_{i h}^{-1}\right)|J(0)| d \xi_{1} d \xi_{2} d \xi_{3}
\end{aligned}
$$

que, integrada, resulta:

$$
K_{M N}=(\kappa)_{E} \frac{1}{\Omega_{E}} \mathcal{A}_{i i_{M N}}
$$

\subsubsection{Matrices $\left[E_{i}\right]$ :}

$$
\begin{aligned}
E_{i_{M N}}= & \int_{\Omega_{E}} \eta\left(\sum_{M=1}^{8} \Phi_{M} v_{i_{M}}^{n}\right) \frac{\partial \Phi_{M}}{\partial x_{j}} \frac{\partial \Phi_{N}}{\partial x_{j}} d \Omega+\int_{\Omega_{E}} \eta\left(\sum_{M=1}^{8} \Phi_{M} v_{j}^{n}\right) \frac{\partial \Phi_{M}}{\partial x_{i}} \frac{\partial \Phi_{N}}{\partial x_{j}} d \Omega \\
& +\int_{\Omega_{E}} \lambda\left(\sum_{M=1}^{8} \Phi_{M} v_{M}^{n}\right) \frac{\partial \Phi_{M}}{\partial x_{j}} \frac{\partial \Phi_{N}}{\partial x_{i}} d \Omega \\
& =\left(v_{i}^{n}\right)_{E} \int_{-1}^{1} \int_{-1}^{1} \int_{-1}^{1} \eta\left(\frac{\partial \Phi_{M}}{\partial \xi_{k}} J(0)_{j k}^{-1}\right)\left(\frac{\partial \Phi_{N}}{\partial \xi_{h}} J(0)_{j h}^{-1}\right)|J(0)| d \xi_{1} d \xi_{2} d \xi_{3} \\
& +\left(v_{j}^{n}\right)_{E} \int_{-1}^{1} \int_{-1}^{1} \int_{-1}^{1} \eta\left(\frac{\partial \Phi_{M}}{\partial \xi_{k}} J(0)_{i k}^{-1}\right)\left(\frac{\partial \Phi_{N}}{\partial \xi_{h}} J(0)_{j h}^{-1}\right)|J(0)| d \xi_{1} d \xi_{2} d \xi_{3} \\
& +\left(v_{j}^{n}\right)_{E} \int_{-1}^{1} \int_{-1}^{1} \int_{-1}^{1} \lambda\left(\frac{\partial \Phi_{M}}{\partial \xi_{k}} J(0)_{j k}^{-1}\right)\left(\frac{\partial \Phi_{N}}{\partial \xi_{h}} J(0)_{i h}^{-1}\right)|J(0)| d \xi_{1} d \xi_{2} d \xi_{3}
\end{aligned}
$$

Una vez resueltas las integrales, esta matriz resulta:

$$
E_{i_{M N}}=(\eta)_{E}\left(v_{i}^{n}\right)_{E} \frac{1}{\Omega_{E}} \mathcal{A}_{i i_{M N}}+(\eta)_{E}\left(v_{j}^{n}\right)_{E} \frac{1}{\Omega_{E}} \mathcal{A}_{i j_{M N}}+(\lambda)_{E}\left(v_{j}^{n}\right)_{E} \frac{1}{\Omega_{E}} \mathcal{A}_{j i}{ }_{M N}
$$

\subsubsection{Matrices $[P]$ :}

$$
P_{M N}=\frac{\Delta t_{i n}}{2} \int_{\Omega_{E}} \frac{\partial \Phi_{M}}{\partial x_{i}} \frac{\partial \Phi_{N}}{\partial x_{i}} d \Omega
$$

Estas matrices tienen la misma forma que las matrices $[K]$ por lo cual su forma, una vez integrada, resulta obvia: 


$$
P_{M N}=\frac{\Delta t_{i n}}{2} \frac{1}{\Omega_{E}} \mathcal{A}_{i i_{M N}}=\alpha_{i i_{M N}}
$$

\subsubsection{Matrices $\left[H_{i}\right]$ :}

$$
H_{i_{M N}}=\frac{\Delta t_{i n}}{2} \int_{\Omega_{E}}\left(\sum_{L=1}^{8} \frac{\partial \Phi_{L}}{\partial x_{j}} v_{j}^{n}{ }_{L}\right) \Phi_{M} \frac{\partial \Phi_{N}}{\partial x_{i}} d \Omega
$$

Nuevamente, el factor escalar entre paréntesis podría simplificarse tomando funciones de interpolación en el centro del elemento. Operando, el resultado sería el siguiente:

$$
\sum_{L=1}^{8} \frac{\partial \Phi_{L}}{\partial x_{j}} v_{j}^{n} \cong\left(\frac{\partial v_{j}^{n}}{\partial x_{j}}\right)_{E}=\frac{1}{8} \sum_{N=1}^{8} \xi_{k_{L}} J(0)_{j k}^{-1} v_{j}^{n}
$$

con lo cual, la derivada calculada como constante en el elemento podría salir de la integral, y la integral resultante tendría la forma de las matrices $\left[B_{i}\right]$, es decir:

$$
H_{i_{M N}}=\frac{\Delta t_{i n}}{2}\left(\frac{\partial v_{j}^{n}}{\partial x_{j}}\right)_{E} B_{i_{M N}}
$$

Sin embargo, dado que se ha considerado a $v_{j}^{n}$ constante en el elemento, estas integrales pueden considerarse nulas puesto que involucran derivadas de las componentes de velocidad que, para estas matrices estabilizadoras, se adoptan constantes.

\subsection{INTEGRACIÓN ANALÍTICA DE MATRICES ELEMENTALES 2: $\boldsymbol{J}\left(\xi_{1}, \xi_{2}, \xi_{3}\right)$.}

Según se indicó anteriormente, la segunda estrategia de integración consiste en calcular las matrices de masa $[M]$ y $\left[M_{D}\right]$ y matrices convectivas $\left[B_{i}\right]$ en forma exacta, sin tomar los jacobianos en el centro del elemento sino considerando la dependencia del Jacobiano con la posición, y sólo las matrices difusivas $\left[D_{i j}\right],\left[E_{i}\right]$ y $[K]$ y las matrices de estabilización, como por ejemplo la matriz $\left[C_{i}\right]$, se calculan tomando los jacobianos en el centro del elemento.

Las matrices $[M],\left[B_{i}\right]$ y $\left[M_{D}\right]$ se resuelven utilizando el software de matemáticas simbólicas MAXIMA (http://maxima.sourceforge.net/es/index.html) en forma completa, o exacta. Esta implementación se hizo con la intención de mejorar aún más la aproximación, resolviendo las integrales en forma exacta para los términos advectivos y para la matriz de masa. La matriz de masa diagonal se obtiene sumando los elementos de cada línea de la matriz de masa consistente y ubicándolos en la diagonal principal. 
Esta estrategia de integración de matrices se plantea aquí como una prueba interesante y se evaluará en un problema de aplicación, pero podrían hacerse más pruebas con esta metodología como, por ejemplo, examinar los efectos de ambas formas de integrar cuando los elementos de la malla están distorsionados, o probar integrando en forma exacta también las matrices difusivas y de estabilización. En todo caso, lo realizado aquí no agota ni mucho menos las posibilidades de investigación sobre este tema puntual, pero al menos muestra que es una estrategia factible de implementar. Queda para desarrollos ulteriores profundizar en los beneficios de desarrollar técnicas de integración analítica exacta utilizando software de matemáticas simbólicas.

A modo de ejemplo, se muestra a continuación un listado de los comandos utilizados en MAXIMA para obtener las matrices $\left[B_{i}\right]$ :

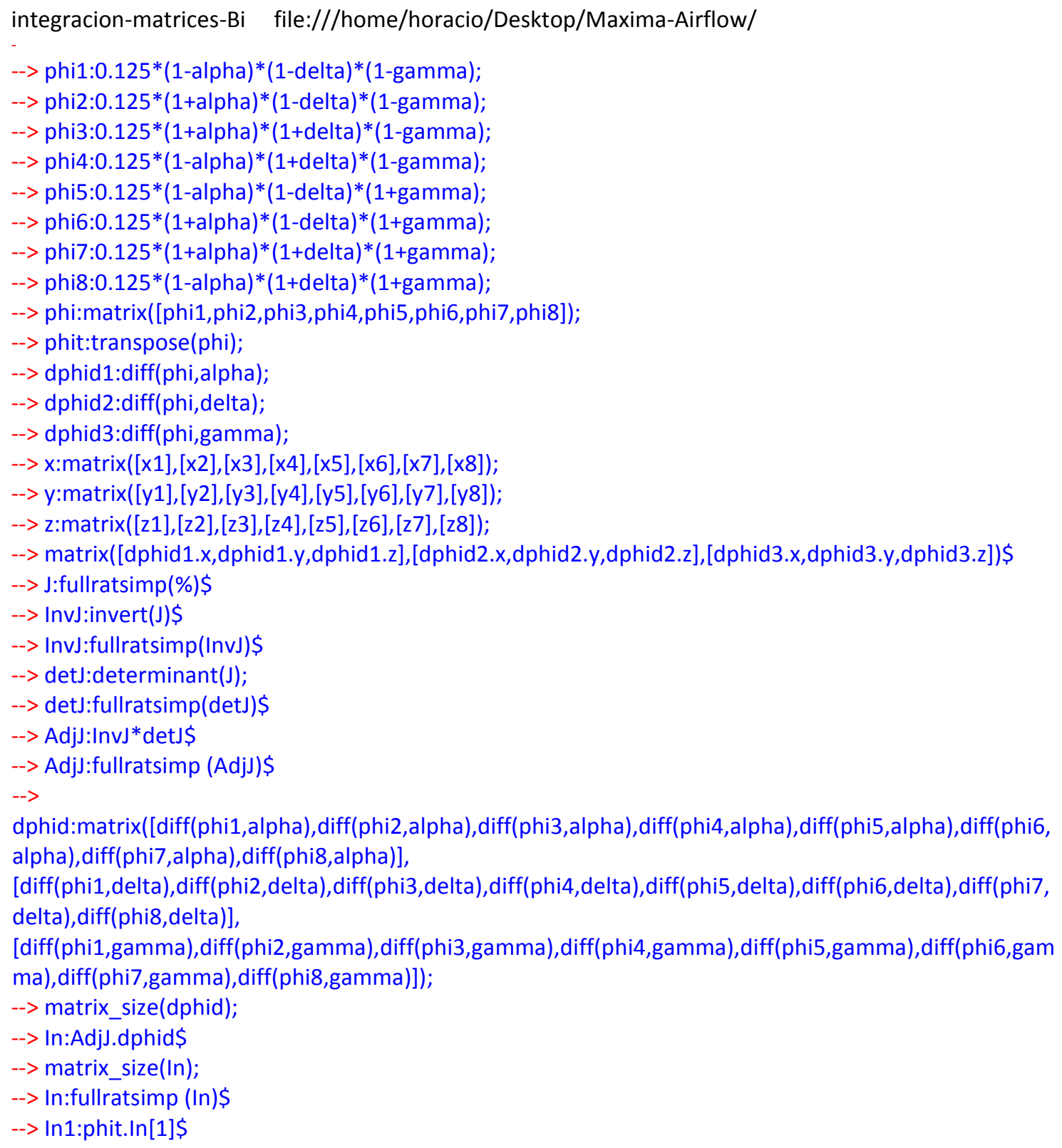


--> matrix_size $(\ln 1)$;

--> In1:fullratsimp $(\ln 1) \$$

$-->\ln 2:$ phit. $\ln [2] \$$

--> matrix_size $(\ln 2)$;

--> In2:fullratsimp $(\ln 2) \$$

$-->\ln 3:$ phit.In[3]\$

--> matrix_size $(\ln 3)$;

--> In3:fullratsimp $(\ln 3) \$$

--> Integ11:integrate(In1,alpha,-1,1)\$

--> Integ12:integrate(Integ11,delta,-1,1)\$

--> BB1:integrate(Integ12,gamma,-1,1)\$

--> matrix_size(BB1);

--> BB1:fullratsimp(BB1);

--> Integ21:integrate(In2,alpha,-1,1)\$

--> Integ22:integrate(Integ21,delta,-1,1)\$

--> BB2:integrate(Integ22,gamma,-1,1)\$

--> BB2:fullratsimp(BB2);

--> matrix_size(BB2);

--> Integ31:integrate(In3,alpha,-1,1)\$

--> Integ32:integrate(Integ31,delta,-1,1)\$

--> BB3:integrate(Integ32,gamma,-1,1)\$

--> BB3:fullratsimp(BB3)\$

--> matrix_size(BB3);

--> L1:transpose(BB1)\$

$-->$ L2:transpose(BB2)\$

--> N2:BB2-L2;

--> L3:transpose(BB3)\$

--> N3:BB3-L3;

--> fortran(BB2[1,1]);

--> fortran(BB2[1,2]);

--> fortran(BB2[1,3]);

--> fortran(BB2[1,4]);

--> fortran(BB2[1,5]);

--> fortran(BB2[1,6]);

--> fortran(BB2[1,7]);

--> fortran(BB2[1,8]);

--> fortran(BB2[2,1]);

--> fortran(BB2[2,2]);

--> fortran(BB2[7,8]);

--> fortran(BB2[8,1]);

--> fortran(BB2[8,2]);

--> fortran(BB2[8,3]);

--> fortran(BB2 $[8,4])$;

--> fortran(BB2[8,5]);

--> fortran(BB2[8,6]);

--> fortran(BB2[8,7]);

--> fortran(BB2[8,8]);

Created with wxMaxima.

Ejecutando el listado de comandos mostrado anteriormente pueden obtenerse los elementos $B_{2}$ de la matriz $\left[B_{2}\right]$ que, una vez implementados en el código Fortran, lucen como se muestra en la imagen a continuación: 


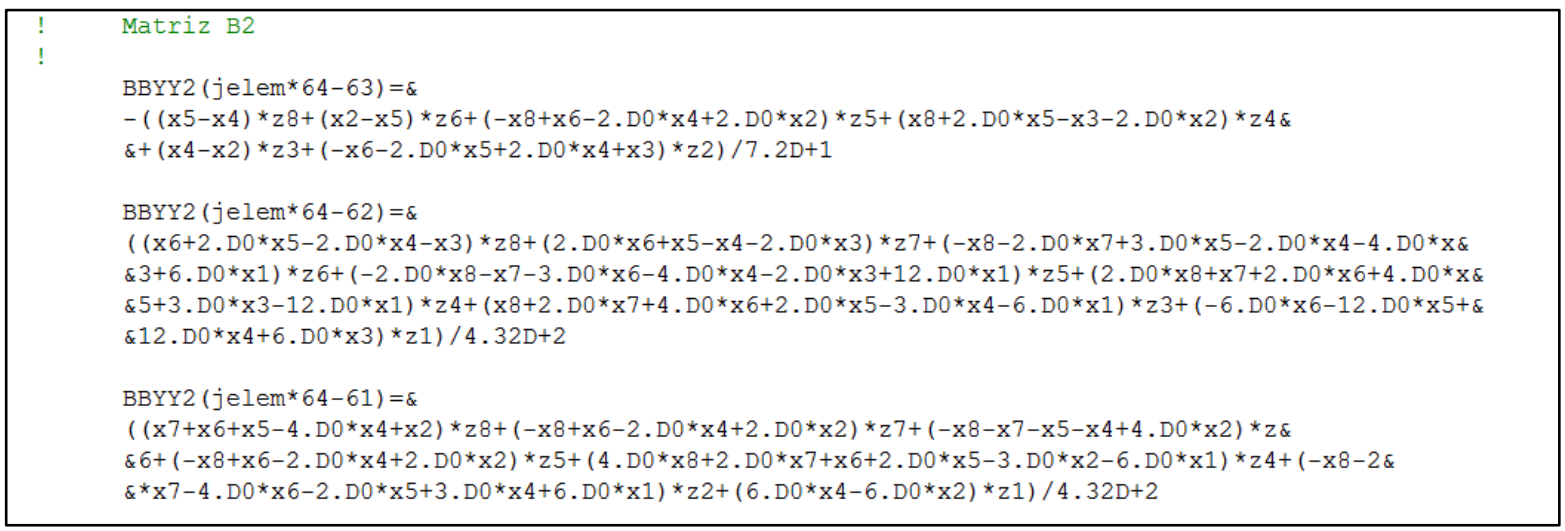

Figura 4.3: Codificación de los elementos $B_{211}, B_{212}$ y $B_{2_{13}}$ de la matriz $\left[B_{2}\right]$.

Como se ve en la imagen, el cálculo de los elementos de estas matrices es directo, conociendo las coordenadas de los ocho nodos del elemento pueden obtenerse las matrices con estas expresiones. La codificación resulta larga, dado que, en los casos de las matrices no diagonales, hay que expresar 64 elementos de la matriz. Para el caso de la matriz de masa diagonal son sólo ocho elementos, pero los mismos resultan largos ya que cada uno de ellos está compuesto por más términos. Sin embargo, desde el punto de vista de ahorro de tiempo de cómputo, esta forma de calcular las matrices es eficiente, dado que se evita la utilización de lazos anidados que suelen presentar problemas a la hora de vectorizar y paralelizar los códigos.

\subsection{TRATAMIENTO DE LAS INTEGRALES DE CONTORNO}

En el capítulo 3 se obtuvieron algunos vectores de contorno. En todos los algoritmos presentados deben calcularse las siguientes integrales:

$$
\begin{gathered}
\left\{f_{j}\right\}^{n}=\int_{\Gamma_{E}}\left[\Phi^{*}\right]^{T} \tau_{i j}^{n} n_{i} d \Gamma \\
\{q\}^{n}=\int_{\Gamma_{E}}\left[\Phi^{*}\right]^{T}\left(\tau_{i j}^{n} v_{j}^{n}\right) n_{i} d \Gamma+\int_{\Gamma_{E}}\left[\Phi^{*}\right]^{T}\left(\kappa \frac{\partial u^{n}}{\partial x_{i}}\right) n_{i} d \Gamma
\end{gathered}
$$

mientras que, en particular para el método CBS, se tenía:

$$
\{h\}^{n}=\frac{\Delta t_{\text {in }}}{2} \int_{\Gamma_{E}}\left[\phi^{*}\right]^{T}\left(\frac{\partial p}{\partial x_{i}}\right) n_{i} d \Gamma
$$

En todos los casos, $\left[\Phi^{*}\right]$ es la matriz de funciones de interpolación evaluadas en la cara de contorno del elemento, que se puede expresar de la siguiente manera: 


$$
\Phi_{N}^{*}= \begin{cases}0 & \text { si } N \text { no es nodo de contorno } \\ \frac{1}{4}\left[1+\eta_{1} \eta_{1_{N}}\right]\left[1+\eta_{2} \eta_{2_{N}}\right] & \text { si } N \text { es nodo de contorno }\end{cases}
$$

donde $N=1,2,3, \ldots, 8$.

En las expresiones (4.70) a (4.72), $n_{i}$ representa las componentes del vector normal al contorno y el factor restante siempre es una acción activa en la superficie del contorno. Así, por ejemplo, $\tau_{i j}$ es una fuerza por unidad de área y $\left(\kappa \partial u^{n} / \partial x_{i}\right)$ es una acción térmica.

En el capítulo 3 se expresaron los vectores equivalentes a las acciones de contorno, luego de que las variables fueron reemplazadas por sus correspondientes variables aproximadas. Según lo indicado en ese capítulo, se tenía:

$$
\begin{gathered}
\left\{f_{j}\right\}^{n}=\int_{\Gamma_{E}}\left[\Phi^{*}\right]^{T}\left[\eta\left(\frac{\partial[\phi]}{\partial x_{i}}\left\{v_{j}\right\}^{n}+\frac{\partial[\phi]}{\partial x_{j}}\left\{v_{i}\right\}^{n}\right)+\lambda\left(\frac{\partial[\phi]}{\partial x_{k}}\left\{v_{k}\right\}^{n}\right) \delta_{i j}\right] n_{i} d \Gamma \\
\{q\}^{n}=\int_{\Gamma_{E}}\left[\Phi^{*}\right]^{T}\left([\Phi]\left\{v_{j}\right\}^{n}\right)\left[\eta\left(\frac{\partial[\phi]}{\partial x_{i}}\left\{v_{j}\right\}^{n}+\frac{\partial[\phi]}{\partial x_{j}}\left\{v_{i}\right\}^{n}\right)+\lambda\left(\frac{\partial[\phi]}{\partial x_{k}}\left\{v_{k}\right\}^{n}\right) \delta_{i j}\right] n_{i} d \Gamma \\
+\int_{\Gamma_{E}}\left[\Phi^{*}\right]^{T} \kappa\left(\frac{\partial[\phi]}{\partial x_{k}}\{u\}^{n}\right) n_{k} d \Gamma \\
\{h\}^{n}=\frac{\Delta t_{i n}}{2} \int_{\Gamma_{E}}\left[\phi^{*}\right]^{T}\left(\frac{\partial[\phi]}{\partial x_{i}}\{p\}^{n}\right) n_{i} d \Gamma
\end{gathered}
$$

Pero para simplificar la obtención de estos vectores de contorno, se toman el valor medio de cada una de las acciones activas en el elemento y se considera que éste es el que actúa en la cara de contorno. Dado que hay cuatro nodos que pertenecen a la cara de contorno del elemento, esa acción da como resultado una distribución uniforme entre los cuatro nodos de la cara de contorno del elemento. Por lo tanto, en cada nodo de contorno actúa una acción que es igual a la cuarta parte de la media de esa acción en el elemento.

Estas simplificaciones representan una pérdida de precisión, ya que se están tomando los valores medios de cantidades que sí presentan variaciones en el elemento. Pero estas integrales actúan únicamente en los contornos en los que aparecen aquellas acciones y no tienen ningún impacto directo dentro del dominio. Por lo tanto, si bien es necesario poner alguna acción en el contorno del dominio discretizado, como han demostrado los resultados obtenidos en el presente estudio, estas acciones no necesitan mayor precisión que la presentada con estas simplificaciones. 
Así, los respectivos vectores equivalentes a las acciones de contorno se obtienen de la siguiente manera:

$$
\begin{gathered}
\left\{f_{j}\right\}^{n}=\frac{1}{4}\left(\tau_{i j}^{n}\right)_{E} n_{i}\{U\} \int_{\Gamma_{E}} d \Gamma \\
\{q\}^{n}=\frac{1}{4}\left(v_{j}^{n} \tau_{i j}^{n}\right)_{E} n_{i}\{U\} \int_{\Gamma_{E}} d \Gamma+\frac{1}{4} \kappa\left(\frac{\partial u^{n}}{\partial x_{i}}\right)_{E} n_{i}\{U\} \int_{\Gamma_{E}} d \Gamma \\
\{h\}^{n}=\frac{\Delta t_{i n}}{2}\left(\frac{\partial p^{n}}{\partial x_{i}}\right)_{E} n_{i}\{U\} \int_{\Gamma_{E}} d \Gamma
\end{gathered}
$$

donde:

$$
\left(\tau_{i j}^{n}\right)_{E}=\eta\left[\left(\frac{\partial v_{i}^{n}}{\partial x_{j}}\right)_{E}+\left(\frac{\partial v_{j}^{n}}{\partial x_{i}}\right)_{E}\right]+\lambda\left(\frac{\partial v_{k}^{n}}{\partial x_{k}}\right)_{E}
$$

son los promedios de las tensiones viscosas en el elemento, mientras que los promedios de las componentes de velocidad vienen dados por:

$$
\begin{gathered}
\left(v_{j}^{n}\right)_{E}=\frac{1}{8} \sum_{N=1}^{8} v_{j}^{n} \\
\left(\frac{\partial v_{j}^{n}}{\partial x_{i}}\right)_{E}=\frac{1}{8} \sum_{N=1}^{8} \xi_{k_{L}} J(0)_{i k}^{-1} v_{j}^{n} \\
\left(\frac{\partial u^{n}}{\partial x_{i}}\right)_{E}=\frac{1}{8} \sum_{N=1}^{8} \xi_{k_{N}} J(0)_{i k}^{-1} u_{N}^{n} \\
\left(\frac{\partial p^{n}}{\partial x_{i}}\right)_{E}=\frac{1}{8} \sum_{N=1}^{8} \xi_{k_{N}} J(0)_{i k}^{-1} p_{N}^{n}
\end{gathered}
$$

Por último:

$$
\begin{gathered}
\Gamma_{E}=\int_{\Gamma_{E}} d \Gamma ; \quad\{U\}^{T}=\left\{\begin{array}{llll}
U_{1} & U_{2} & \ldots & U_{8}
\end{array}\right\} \\
\text { con } U_{N}= \begin{cases}1 & \text { si } N \text { es nodo de contorno } \\
0 & \text { si } N \text { no es nodo de contorno }\end{cases}
\end{gathered}
$$

siendo $\Gamma_{E}$ el área de la cara de contorno del elemento. 
4.6 VECTORES $\{Q\}^{n}$ y $\left\{Q v_{i}\right\}^{n}$ EN LA ECUACIÓN DE TRANSPORTE DE SPALARTALLMARAS.

En el conjunto de expresiones (3.37) se habían definido dos vectores nodales para la ecuación de transporte de $\tilde{v}$ de SA cuya forma de cálculo conviene explicitar. Ellos son los siguientes:

$$
\begin{aligned}
\{Q\}^{n}= & -c b_{1}\left(S^{n}\right)_{E}\{\rho \tilde{v}\}^{n}+c w_{1}\left(f_{w}^{n}\right)_{E}\left\{\rho\left(\frac{\tilde{v}}{d}\right)^{2}\right\}^{n} \\
& -\frac{c b_{2}}{\sigma}\left(\rho^{n}\right)_{E}\left(\frac{\partial \tilde{v}^{n}}{\partial x_{i}}\right)_{E}\left(\frac{\partial \tilde{v}^{n}}{\partial x_{i}}\right)_{E}\{I\} \\
& +\frac{1}{\sigma}\left[\left(v^{n}\right)_{E}+\left(\tilde{v}^{n}\right)_{E}\right]\left(\frac{\partial \rho^{n}}{\partial x_{i}}\right)_{E}\left(\frac{\partial \tilde{v}^{n}}{\partial x_{i}}\right)_{E}\{I\} \\
\left\{Q v_{i}\right\}^{n}=- & c b_{1}\left(S^{n}\right)_{E}\left\{\rho \tilde{v} v_{i}\right\}^{n}+c w_{1}\left(f_{w}{ }^{n}\right)_{E}\left\{\rho\left(\frac{\tilde{v}}{d}\right)^{2} v_{i}\right\}^{n} \\
& -\frac{c b_{2}}{\sigma}\left(\rho^{n}\right)_{E}\left(\frac{\partial \tilde{v}^{n}}{\partial x_{i}}\right)_{E}\left(\frac{\partial \tilde{v}^{n}}{\partial x_{i}}\right)_{E}\left\{v_{i}\right\} \\
& +\frac{1}{\sigma}\left[\left(v^{n}\right)_{E}+\left(\tilde{v}^{n}\right)_{E}\right]\left(\frac{\partial \rho^{n}}{\partial x_{i}}\right)_{E}\left(\frac{\partial \tilde{v}^{n}}{\partial x_{i}}\right)_{E}\left\{v_{i}\right\}
\end{aligned}
$$

Como se ve, por simplicidad se han adoptado varios promedios de elemento, cuyas expresiones expandidas son las siguientes:

$$
\begin{gathered}
\left(S^{n}\right)_{E}=\frac{1}{8} \sum_{N=1}^{8} S_{N}^{n} \\
\left(\tilde{v}^{n}\right)_{E}=\frac{1}{8} \sum_{N=1}^{8} \tilde{v}_{N}^{n} ; \quad\left(v^{n}\right)_{E}=\frac{1}{8} \sum_{N=1}^{8}\left(\frac{\mu}{\rho}\right)_{N}^{n} \\
\left(\rho^{n}\right)_{E}=\frac{1}{8} \sum_{N=1}^{8} \rho_{N}^{n} ;\left(f_{w}{ }^{n}\right)_{E}=\frac{1}{8} \sum_{N=1}^{8} f_{w_{N}}^{n} \\
\left(\frac{\partial \rho}{\partial x_{i}}\right)_{E}=\frac{1}{8} \sum_{N=1}^{8} \xi_{k_{N}} J(0)_{i k}^{-1} \rho_{N}^{n} \\
\left(\frac{\partial \tilde{v}^{n}}{\partial x_{i}}\right)_{E}=\frac{1}{8} \sum_{N=1}^{8} \xi_{k_{N}} J(0)_{i k}^{-1} \tilde{v}_{N}^{n}
\end{gathered}
$$

donde los valores de $S$ y $f_{w}$ se calculan como se indica en las expresiones (2.44). 


\subsection{CONTROL DE MODOS ESPURIOS}

La integración de matrices elementales usando un único punto de cuadratura puede conducir a la aparición de modos espurios conocidos como modos de Hourglass. Con respecto a la integración analítica hecha calculando la matriz jacobiana en el centro del elemento (primera estrategia de integración desarrollada en este trabajo) de la lectura de la referencia [25] se puede concluir que estos modos espurios no debieran aparecer. Sin embargo, en dicha referencia esto no ha sido matemáticamente demostrado, por lo cual se implementó aquí un método de control de modos espurios el cual puede activarse o desactivarse con un parámetro determinado en los archivos de entrada.

En tres dimensiones, cuando se utiliza un único punto de cuadratura, hay cuatro modos espurios que, cuando se excitan numéricamente, pueden permanecer sin ningún tipo de amortiguación y cambiar la solución física del problema. Un método de control de estos modos llamado "h Stabilization" consiste en agregar a las matrices difusivas la siguiente matriz:

$$
[\mathrm{HG}]=\varepsilon_{h g} \eta\left[\begin{array}{llll}
\left\{\Gamma_{1}\right\} & \left\{\Gamma_{2}\right\} & \left\{\Gamma_{3}\right\} & \left\{\Gamma_{4}\right\}
\end{array}\right]\left[\begin{array}{cccc}
C_{1} & 0 & 0 & 0 \\
0 & C_{2} & 0 & 0 \\
0 & 0 & C_{3} & 0 \\
0 & 0 & 0 & C_{4}
\end{array}\right]\left[\begin{array}{l}
\left\{\Gamma_{1}\right\}^{T} \\
\left\{\Gamma_{2}\right\}^{T} \\
\left\{\Gamma_{3}\right\}^{T} \\
\left\{\Gamma_{4}\right\}^{T}
\end{array}\right]
$$

donde $\operatorname{los}\left\{\Gamma_{i}\right\}$ son los vectores de Hourglass, dados por:

$$
\begin{aligned}
& \left\{\Gamma_{1}\right\}^{T}=\left\{\begin{array}{llllllll}
1 & 1 & -1 & -1 & -1 & -1 & 1 & 1
\end{array}\right\} \\
& \left\{\Gamma_{2}\right\}^{T}=\left\{\begin{array}{llllllll}
1 & -1 & -1 & 1 & -1 & 1 & 1 & -1
\end{array}\right\} \\
& \left\{\Gamma_{3}\right\}^{T}=\left\{\begin{array}{llllllll}
1 & -1 & 1 & -1 & 1 & -1 & 1 & -1
\end{array}\right\} \\
& \left\{\Gamma_{4}\right\}^{T}=\left\{\begin{array}{llllllll}
-1 & 1 & -1 & 1 & 1 & -1 & 1 & -1
\end{array}\right\}
\end{aligned}
$$

El factor $\varepsilon_{h g}$ se toma igual a uno; además:

$$
C_{1}=C_{1}=C_{1}=C_{1}=\bar{h} \sqrt[3]{\Omega_{E}} ; \quad \bar{h}=\frac{1}{2}\left(1-\sqrt[3]{\frac{\Omega_{E, \min }}{\Omega_{E, \max }}}\right)
$$

donde $\Omega_{E, \min }$ y $\Omega_{E, \max }$ representan los volúmenes mínimo y máximo elegidos entre todos los elementos de malla. Se pueden encontrar detalles adicionales sobre el control de los modos espurios en Gresho et al. ${ }^{[24]}$. 


\section{ASPECTOS IMPORTANTES DE LA IMPLEMENTACIÓN.}

\subsection{CAPTURA DE CHOQUE.}

Con el objetivo de capturar las fuertes discontinuidades y eliminar oscilaciones de alta frecuencia cerca de las ondas de choque, se utiliza un conocido método que adiciona viscosidad artificial selectivamente sólo en las regiones del dominio con fuertes gradientes de presión ${ }^{[50]}$. La solución suavizada se obtiene a partir de la solución no suavizada utilizando la siguiente expresión

$$
\left\{\boldsymbol{U}_{s}\right\}^{n+1}=\{\boldsymbol{U}\}^{n+1}+\left[M_{D}\right]^{-1}\{\boldsymbol{D}\}^{n}
$$

donde el vector de amortiguamiento ficticio viene dado por:

$$
\{\boldsymbol{D}\}^{n}=\sum_{E=1}^{N E M} C_{E} C_{A D} S_{E}\left([M]-\left[M_{D}\right]\right)_{E}\{\boldsymbol{U}\}^{n}
$$

En (5.2) $N E M$ es la cantidad de elementos de la malla, $C_{E}=\Delta t / \Delta t_{E}$ es el número de Courant local, $C_{A D}$ es un coeficiente de viscosidad artificial dado por el usuario (en este trabajo se adoptaron valores y $0.0 \leq C_{A D} \leq 2.0$ dependiendo del caso) y $S_{E}$ es un sensor de presiones a nivel de elemento obtenido como un promedio de los valores nodales $S_{N}$ del elemento. Los valores $S_{N}$ son componentes del siguiente vector global

$$
\{S\}^{n}=\frac{\sum_{E}\left|\left([M]-\left[M_{D}\right]\right)_{E}\{p\}_{E}^{n}\right|}{\sum_{E}\left|\left([M]-\left[M_{D}\right]\right)_{E}\right|\{p\}_{E}^{n}}
$$

donde las barras indican valor absoluto y $[\mathrm{M}]$ es la matriz de masa consistente del elemento

$$
[M]=\int_{\Omega_{E}}[\phi]^{T}[\phi] d \Omega
$$

\subsection{CONVERGENCIA:}

\subsubsection{Convergencia de los términos iterativos:}

La convergencia de los términos iterativos es obtenida cuando las siguientes condiciones son satisfechas simultáneamente: 


$$
\begin{gathered}
r_{I+1}^{\rho}=\left[\frac{\sum_{N}\left|\rho_{I+1}-\rho_{I}\right|^{2}}{\sum_{N} \rho_{I}^{2}}\right]^{\frac{1}{2}} \leq T o \\
r_{I+1}^{\rho v}=\left[\frac{\sum_{N}\left|\rho v_{i I+1}-\rho v_{i I}\right|^{2}}{\sum_{N} \rho v_{i I}^{2}}\right]^{\frac{1}{2}} \leq T o \\
r_{I+1}^{\rho e}=\left[\frac{\sum_{N}\left|\rho e_{I+1}-\rho e_{I}\right|^{2}}{\sum_{N} \rho e_{I}^{2}}\right]^{\frac{1}{2}} \leq T o
\end{gathered}
$$

donde, $I$ significa iteración anterior e $I+1$ significa iteración actual, $N$ es un índice de nodos $(N=1,2,3 \ldots, n n m)$ y $n n m$ es la cantidad total de nodos de la malla de elementos finitos. La tolerancia adoptada en este trabajo es $T o=1 * 10^{-3}$, pero la misma puede ser fijada en valores diferentes.

Para la primera comparación de un nuevo paso de tiempo, dado que para este último no se tiene un valor de "iteración anterior", se adopta el valor convergido del paso de tiempo anterior como el valor correspondiente a la iteración anterior.

Como se indicó en capítulos anteriores, este proceso iterativo toma lugar en el esquema TG y MTG, mientras que no se utiliza en el método CBS o CBS single-step. Con los valores de tolerancia utilizados aquí $\left(T o=1 * 10^{-3}\right)$ en problemas donde se busca un estado estacionario estas iteraciones toman lugar durante unos pocos pasos de tiempo, la cantidad de iteraciones por paso de tiempo decae muy rápidamente y se reduce a cero.

Se dice, entonces, que hay "cero iteraciones" cuando todos los residuos calculados en (5.5), (5.6) y (5.7) están por debajo del valor de tolerancia ya en la primera comparación dentro del nuevo paso de tiempo, cuando se comparan los valores recién calculados para el nuevo paso de tiempo con los valores convergidos del paso de tiempo anterior. En este caso no es necesario cerrar el lazo iterativo recalculando nuevos valores de iteración, y los valores convergidos del nuevo paso de tiempo son los últimos calculados (en este caso se dice que hay "cero iteraciones" porque, estrictamente no se llega a completar un lazo iterativo y se sale del mismo sin siquiera recalcular valores de iteración ni una sola vez).

\subsubsection{Convergencia a estado estacionario o alcance del tiempo de simulación estipulado:}

El proceso de solución finaliza cuando la variable $t$ (tiempo) alcanza un valor predeterminado, previamente establecido por el usuario, o cuando se llega al estado estacionario. Esta última condición se considera cumplida cuando la siguiente expresión: 


$$
R^{n+1}=\left[\sum_{N}\left|\rho^{n+1}-\rho^{n}\right|^{2}\right]^{\frac{1}{2}} \leq T O L_{t}
$$

se satisface durante un cierto número de pasos de tiempo.

En la expresión (5.8) $R$ es el residuo de densidad, que típicamente corresponde a la ecuación de continuidad o conservación de la masa, $n$ indica paso de tiempo anterior y $n+1$ indica paso de tiempo corriente. Los valores de tolerancia suelen ser de TOLt $=10^{-3}$, TOLt $=$ $10^{-4}$ o $T O L_{t}=10^{-5}$.

Es importante tener en cuenta que estos esquemas recurrentes de avance en el tiempo, como los que se observan en el esquema TG o el método CBS, pueden ser utilizados tanto para hacer una simulación transitoria como para llegar a un estado estacionario a partir de una condición inicial. En este último caso los pasos de tiempo no representan pasos de tiempo físico sino, más bien, pasos de avance hacia la convergencia de estado estacionario siendo, la evolución entre el estado inicial y el estado estacionario, un transitorio ficticio sin sentido físico.

Aquí adquiere especial atención la definición del paso de tiempo y la necesidad (o no) de adoptar un único paso de tiempo para toda la malla cuando, en realidad, de la expresión (3.38) se sabe que, en estos algoritmos explícitos que son condicionalmente estables, cada elemento tiene un paso de tiempo de estabilidad propio.

En un cálculo transitorio es evidente que existe la necesidad de que todos los valores nodales de la malla sean actualizados para cada paso de tiempo físico, el cual ha de ser sin dudas el menor de toda la malla para que haya estabilidad. Existe, en este caso, una forma de evitar la ineficiencia que significa estar calculando la solución para toda la malla con el paso de tiempo más pequeño de la misma, conocida como Sub-Cycling. Esta técnica se menciona aquí pero no fue utilizada en este trabajo. Sin embargo, la misma ya fue utilizada con éxito en implementaciones similares a las realizadas en este trabajo. Para conocer los detalles de esta técnica, específicamente aplicada a implementaciones del esquema de Taylor-Garlekin y en un contexto de paralelización como el utilizado en este trabajo, se recomienda la lectura de la referencia [37].

En casos de cálculos de estado estacionario, en principio, sería sencillo utilizar un paso de tiempo local, calculado como el menor en la vecindad de cada nodo, y abandonar la idea de utilizar un paso de tiempo único para toda la malla, dado que aquí el paso de tiempo no tiene significación física y lo que interesa es que la solución converja rápidamente al estado 
estacionario, sin importar los transitorios intermedios. Sin embargo, la mejor forma de hacer esto parece ser, nuevamente, la utilización de las técnicas de Sub-Cycling referidas en el párrafo anterior. La implementación de estas técnicas se encuentra fuera del marco de objetivos de esta tesis y se deja para futuras investigaciones.

Sí es importante señalar que, para el caso de esquemas como el CBS o el MTG donde se utilizan pasos de tiempo exteriores y pasos de tiempo interiores, lo dicho en los párrafos anteriores sólo aplica a los pasos de tiempo exteriores. Los pasos de tiempo interiores actúan únicamente en matrices estabilizadoras, por lo cual pueden ajustarse para obtener la mejor estabilización posible, y esto ocurre cuando se utiliza un paso de tiempo local en dichas matrices.

\subsection{IMPLEMENTACIÓN DE CÓDIGOS PARALELOS.}

\subsubsection{Descripción general de la metodología de paralelización.}

Esta sección tiene por objeto proporcionar al lector información básica sobre las metodologías de paralelización empleadas en este trabajo. Sin embargo, una descripción pormenorizada de estas técnicas, así como las métricas de eficiencia de paralelización y speed up obtenidas, podría demandar una tesis completa, y no constituye el núcleo o foco principal de este trabajo, para el cual la paralelización es más un medio para poder realizar las simulaciones requeridas, que su eje central o foco de gravitación. Para obtener detalles pormenorizados de estas técnicas se recomienda la lectura de la referencia [37].

Partiendo de los códigos iterativos ya disponibles para correr en procesadores vectoriales, los mismos fueron paralelizados para poder correr tanto en ambiente de memoria compartida (computadora con más de un procesador o núcleo de procesamiento para la cual todos los procesadores acceden al mismo banco de memoria RAM) o en ambiente de memoria distribuida ("clusters" de varias computadoras conectadas en red, cada una de las cuales puede tener uno o más núcleos de procesamiento).

Para ello se optó por una programación paralela que pudiese ser utilizada con una mínima dependencia del sistema operacional o de la estructura del hardware. Así, toda la lógica de distribución de tareas, balanceo de cargas de trabajo y organización de la comunicación de los datos entre las computadoras está implementada directamente en el código, sin ninguna otra capa de software de gerenciamiento. 
Todos los códigos fueron implementados en FORTRAN 90. La comunicación de datos entre computadoras se hace utilizando las librerías MPICH de acceso público. Desde la paralelización de los códigos originales en 2008 en el laboratorio de computación CEMACOM de la UFRGS hasta hoy (incluyendo las modificaciones posteriormente introducidas para agregar el modelo de turbulencia, efectuar cambios en la forma de integración y codificar otros algoritmos basados en el método CBS y CBS single-step), fueron utilizados diferentes sistemas operativos como Windows XP, Windows 7, Linux/Debian y Linux/RedHat). Como la única exigencia con relación al sistema operativo es el soporte a la comunicación en red y al API MPI, y dado que tanto FORTRAN 90 como MPI tienen patrones que garantizan su portabilidad, los códigos desarrollados pueden ser utilizados en otras plataformas y otros sistemas operativos. Para el uso de múltiples procesadores o núcleos en una única computadora en ambiente de memoria compartida, el soporte es provisto por los compiladores también a través de la API MPI, exigiendo del sistema operativo apenas el soporte de multiprocesamiento.

Considerando que la red de comunicación existente en laboratorios de investigación universitarios es, en algunos casos, de tasas de transferencia lentas (Fast Ethernet 100Mbps a $1 \mathrm{Gbps})$, la implementación paralela fue hecha de forma de tener granularidad lo más gruesa posible, de manera de minimizar el tiempo de comunicación de datos entre los procesadores comparado con el tiempo de procesamiento, garantizando así una mayor eficiencia de paralelización.

Por las mismas limitaciones, el modelo de programación paralela utilizado es del tipo "hostless program" de modo que todos los procesadores ejecuten el mismo código y que los intercambios de datos sean efectuados directamente por los procesadores unos con los otros sin la intervención de ningún controlador. La opción por este modelo es adecuada para redes relativamente pequeñas con una topología en estrella, como las de computadoras conectadas directamente a un único switch de red, pues descentraliza y agiliza el proceso de comunicación entre los procesadores porque posibilita comunicaciones simultáneas y disminuye el tráfico de datos en la red en comparación a un modelo del tipo "master-slave".

La división de tareas utilizada empleó el particionado nodal de la estructura de datos, basado en el presupuesto de que el esfuerzo computacional asociado a cada nodo de la malla es constante, y también basado en un indicador inicial de la capacidad de procesamiento de cada procesador como, por ejemplo, la frecuencia del procesador. La tarea de división de la malla según su menor dimensión geométrica (para definir las cargas de trabajo de cada 
procesador) fue realizada mediante la división del sistema de ecuaciones según su menor dimensión (ancho de banda). Para esto, se utilizó un tratamiento de malla basado en una única reordenación nodal para minimización del ancho de banda de la matriz del sistema de ecuaciones. Este tratamiento puede ser considerado como una variante del método global de particionado en fajas (stripwise partitioning - STRIP) (ver Dorneles ${ }^{[38]}$ ). Se emplean elementos fantasmas (ghost elements) para minimizar la comunicación entre procesadores a costa de redundancia en el esfuerzo computacional (ver Demkowicz et al. ${ }^{[39]}$ ).

El balanceo de esta carga de trabajo es hecho al inicio del proceso de solución de forma estática corriendo un determinado número de iteraciones y redistribuyendo los nodos de la malla entre procesadores en función del tiempo de procesamiento individual hasta que el equilibrio sea alcanzado. Esta metodología es aplicable en procesos de solución para los cuales el esfuerzo computacional es constante a lo largo de las iteraciones.

La estructura de datos utilizada fue de elemento por elemento o EBE (Element-ByElement) evitando la necesidad de montaje de matrices globales que complican el análisis de problemas grandes por las necesidades de memoria que implican.

\subsubsection{Métricas de los programas paralelos.}

Dado que en la siguiente sección se presentan las métricas de paralelización obtenidas en un caso particular, es necesario definir estas métricas previamente.

Se define como "aceleración" o "ganancia de velocidad" de procesamiento (speed-up) a la siguiente relación ${ }^{[51]}$ :

$$
S_{p}=\frac{t_{s}}{t_{p}}
$$

Donde $p$ es la cantidad de núcleos de procesamiento utilizados en paralelo, $t_{s}$ es el tiempo de procesamiento total transcurrido en una corrida secuencial (en un único núcleo de procesamiento) y $t_{p}$ es el tiempo de procesamiento total transcurrido en la corrida en paralelo con $p$ núcleos de procesamiento. Este speed-up será "absoluto" cuando el tiempo de procesamiento secuencial corresponda al mejor programa secuencial posible, mientras que será "relativo" cuando el tiempo $t_{s}$ corresponda a una corrida hecha con el mismo código paralelo, pero utilizando un único núcleo de procesamiento. A no ser que sea especificado en contrario, debe considerarse que el speed-up ser refiere al speed-up relativo. El speed-up teórico o ideal es aquel para el cual $S_{p}=p$ lo cual indicaría que toda la capacidad computacional es utilizada para acelerar la ejecución del programa. 
Se define como eficiencia de paralelización $E_{p}$ para $p$ núcleos de procesamiento a la siguiente relación:

$$
E_{p}=\frac{S_{p}}{p}=\frac{t_{s}}{p t_{p}}
$$

la cual indica cuánto del speed-up teórico fue alcanzado. Aquí consideramos que cada hilo (thread) del cómputo paralelo es realizado por un núcleo de procesamiento sin importar la configuración específica del hardware (si es un "cluster" con varios nodos o si es una única computadora o nodo; si cada nodo tiene uno o más procesadores; si cada procesador tiene uno o más núcleos).

Pero las expresiones (5.9) y (5.10) son válidas únicamente para el caso de correr en clusters homogéneos o máquinas donde todos los núcleos de procesamiento tienen la misma velocidad o capacidad de cómputo. En caso de que esto último no ocurra, puede calcularse la velocidad relativa de cada procesador de la siguiente manera:

$$
v_{r}^{i}=\frac{t_{s}^{r e f}}{t_{s}^{i}}
$$

Donde $t_{s}^{i}$ es el tiempo de cómputo del procesador $i$ en una corrida secuencial (con un único núcleo de procesamiento) y $t_{s}^{r e f}$ es el tiempo de cómputo del procesador adoptado como referencia, también en una corrida secuencial. De la misma forma, la velocidad relativa de la ejecución en paralelo viene definida por la siguiente expresión:

$$
v_{r}^{p}=\frac{t_{s}^{r e f}}{t_{p}}
$$

La eficiencia de paralelización para $p$ núcleos de procesamiento (no necesariamente homogéneos) es, entonces, la siguiente:

$$
E_{p}=\frac{v_{r}^{p}}{\sum_{i=1}^{p} v_{r}^{i}}
$$

Y el speed-up se calcula como sigue a continuación:

$$
S_{p}=p E_{p}
$$

Por último, se define como "escalabilidad" de un programa a la propiedad de este de mantener la eficiencia de paralelización a medida que se agregan más núcleos de procesamiento al conjunto. Esto significa que $E_{p}$ se mantenga en un valor constante $k$ que no 
depende de la cantidad de núcleos de procesamiento $p$, determinando que la relación (5.14) tome la forma lineal.

$$
S_{p}=k p
$$

5.3.3 Evaluación de eficiencia de paralelización: Flujo supersónico no viscoso alrededor de una esfera

A continuación, se muestra un único ejemplo para mostrar algunas de las métricas obtenidas con las técnicas de paralelización utilizadas en este trabajo. Este ejemplo corresponde a un artículo presentado en 2011 en el Congreso Argentino de Tecnología Aeroespacial ${ }^{[52]}$. El mismo fue efectuado sobre una implementación del esquema de TaylorGalerkin que no incluía el modelo de turbulencia. Sin embargo, y a pesar de que fue realizado en un hardware que podría considerarse obsoleto, éste es útil a los fines de ilustrar las métricas de "speed-up" y "eficiencia de paralelización” obtenidas.

Se considera en este ejemplo un flujo supersónico compresible no viscoso $\gamma=1.4$ pasante por una esfera de radio $\mathrm{R}=1,0$. Solo un octavo de la geometría esférica fue considerado en este caso debido a la simetría geométrica y de flujo, como puede verse en la figura 5.1.
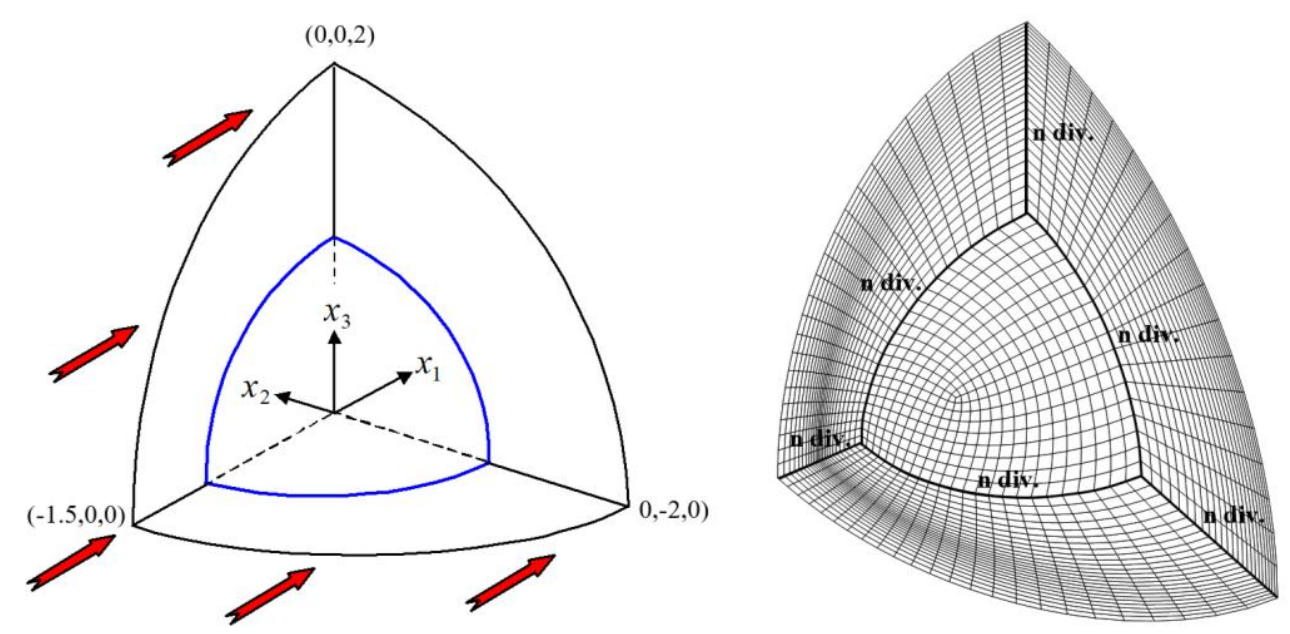

Figura 5.1: Dominio y malla de elementos finitos para obtener métricas de paralelización.

Las condiciones de contorno prescriptas a la entrada son: $v_{1 \infty}=3,0, v_{2 \infty}=v_{3 \infty}=$ 0,0, $e_{\infty}=6,2857, \rho_{\infty}=1.0$ y el número de Mach de referencia es $M_{\infty}=3,0$. La componente del vector velocidad normal al contorno sólido de la esfera fue anulada. Lo mismo se hizo en los contornos (planos) de simetría del dominio. Las condiciones iniciales que se adoptaron en todo el dominio son: $v_{1}{ }^{0}=3,0 ; v_{2}{ }^{0}=v_{3}{ }^{0}=0,0 ; u^{0}=1.7857 ; e^{0}=$ $6,2857 \rho^{0}=1.0$ y $p^{0}=0.71428$. Estos valores se especifican en todos los nodos de la malla 
de elementos finitos, excluyendo aquellos en donde se prescriben condiciones de contorno. Se utiliza un factor de seguridad $\beta=0.2 \mathrm{y}$, así, el paso de tiempo efectivo adimensional resulta $\Delta t=0,4^{*} 10^{-3}$.

Sobre el dominio de análisis fue generada una familia de mallas, cada una correspondiendo a un número diferente de divisiones ( $\mathrm{n}$ div.) de las líneas de definición del dominio según se ve en la figura 5.1. Las características de las mallas se pueden ver en la tabla 5.1.

\begin{tabular}{|l|c|c|c|}
\hline Malla & $\begin{array}{c}\text { Cantidad de } \\
\text { elementos }\end{array}$ & $\begin{array}{c}\text { Cantidad de } \\
\text { nodos }\end{array}$ & $\begin{array}{c}\text { Cantidad de grados de } \\
\text { libertad }\end{array}$ \\
\hline ESF020 & 6.000 & 6.951 & 34.755 \\
\hline ESF030 & 20.250 & 22.351 & 111.755 \\
\hline ESF040 & 48.000 & 51.701 & 258.505 \\
\hline ESF050 & 93.750 & 99.501 & 497.505 \\
\hline ESF060 & 162.000 & 170.251 & 851.255 \\
\hline ESF070 & 257.250 & 268.451 & 1.342 .255 \\
\hline ESF080 & 384.000 & 398.601 & 1.993 .005 \\
\hline ESF090 & 546.750 & 565.201 & 2.826 .005 \\
\hline ESF100 & 750.000 & 772.751 & 3.863 .755 \\
\hline ESF110 & 998.250 & 1.025 .751 & 5.128 .755 \\
\hline
\end{tabular}

Tabla 5.1: Mallas utilizadas para obtener métricas de paralelización. $\left(2011^{[52]}\right)$

Como se ve en la tabla precedente, las mallas obtenidas fueron designadas por ESFxxx, donde xxx representa el número de divisiones empleado.

En la figura 5.2 se muestran los contornos de número de Mach que se obtienen como resultado; se observa que este resultado es muy próximo al que presentan Molina \& Huot ${ }^{\text {[26] }}$ para el mismo caso.
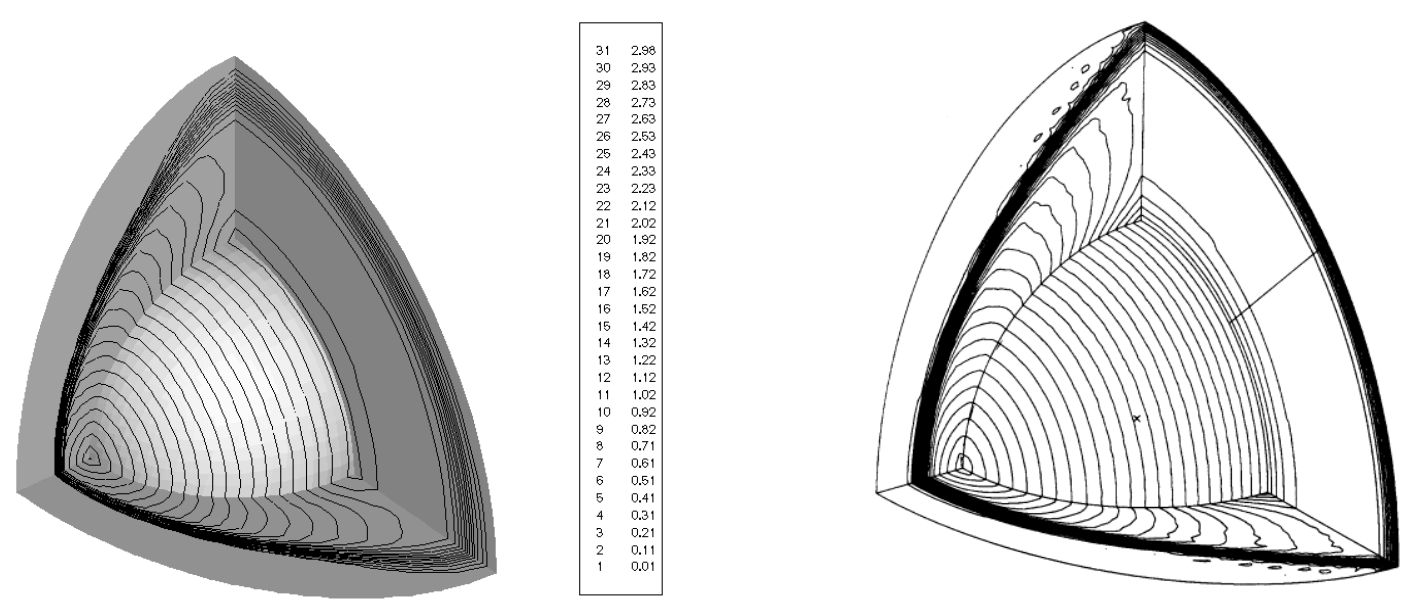

Figura 5.2: Contornos de número de Mach. Izq.: Este trabajo (ESF20). Der.: Molina \& Hout. 
La implementación paralela fue testeada con un conjunto de 11 computadoras en un "cluster" permanente heterogéneo, conectados a través de una red "Fast Ethernet" (100 Mbps) y "Gigabit Ethernet" (1 Gbps). Cada computadora disponía de un procesador con un único núcleo AMD Athlon 64 y 4 GB de memoria RAM. La frecuencia de los núcleos utilizados es de 2,3 GHz en tres de las computadoras, 2,4 GHz en cinco de las computadoras y 2,5 GHz en las tres restantes.

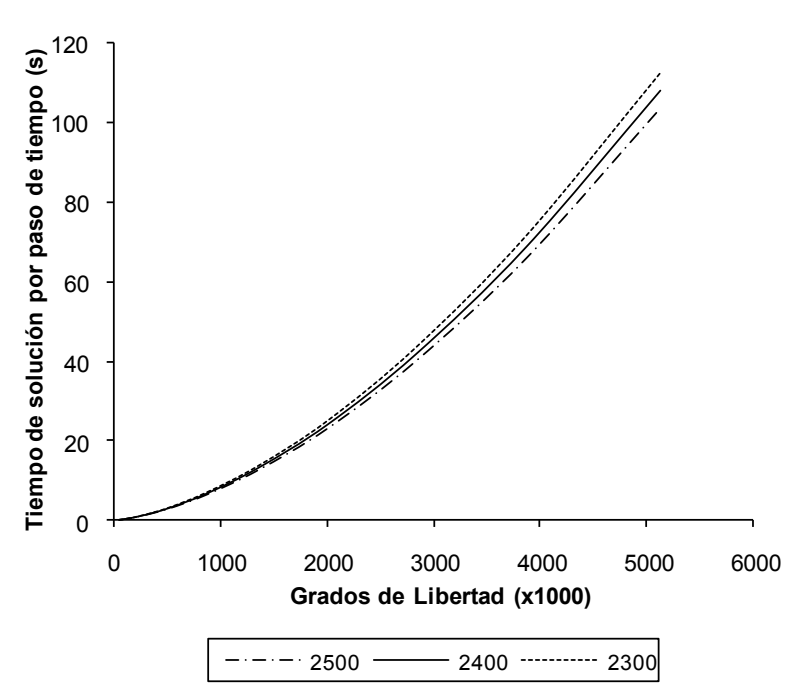

Figura 5.3: Tiempo de solución por paso de tiempo en función del Nro de GL según la frecuencia del procesador.

La división de tareas fue hecha una única reordenación nodal previa con algoritmo minimizador de banda con balanceo de carga de trabajo computacional.

Primeramente, los diversos tamaños de problema (mallas) fueron analizados por cada computadora con un único núcleo de procesamiento en forma secuencial. Dada la memoria disponible en cada computadora este análisis secuencial se restringió hasta el problema ESF090. Los tiempos de solución obtenidos se muestran en la figura 5.3.
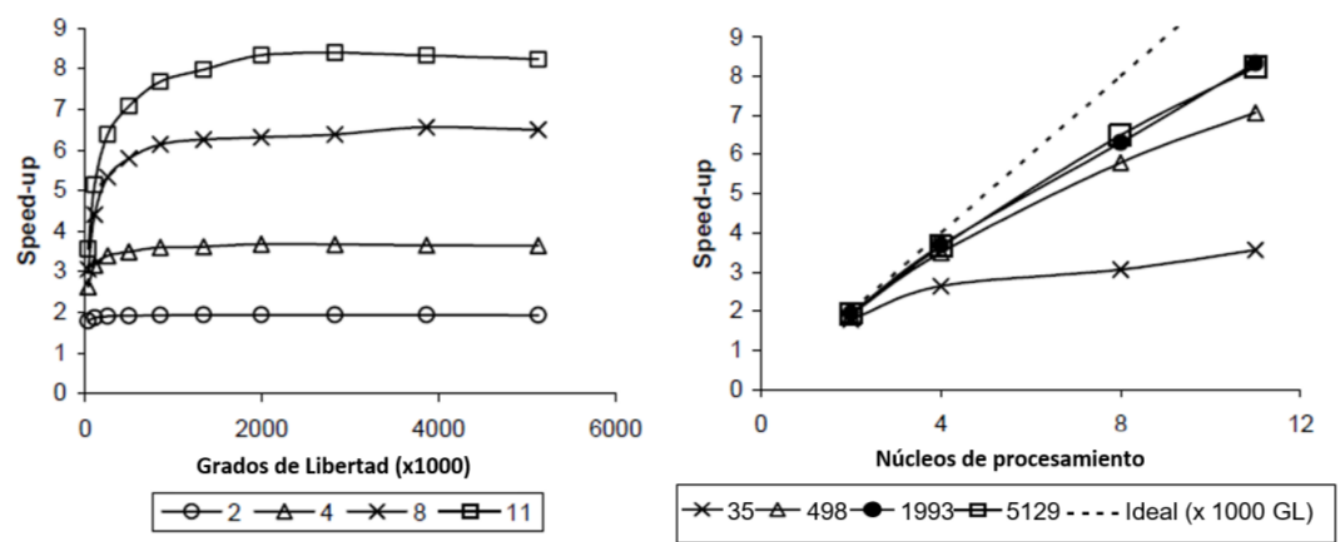

Figura 5.4: "Speed-up" en función del tamaño de malla para diferente cantidad de núcleos (der.) y en función del número de núcleos para diferentes tamaños de malla (izq.). 
A partir del análisis secuencial, fue posible cuantificar el desempeño de la solución paralela. Con el uso de la red de 1 Gbps las computadoras fueron agrupadas en conjuntos de 2, 4, 8 y 11 núcleos de procesamiento para los problemas ESF020 a ESF110, siendo obtenidos los valores de "speed-up" y de eficiencia que se muestran en las figuras 5.4 y 5.5.
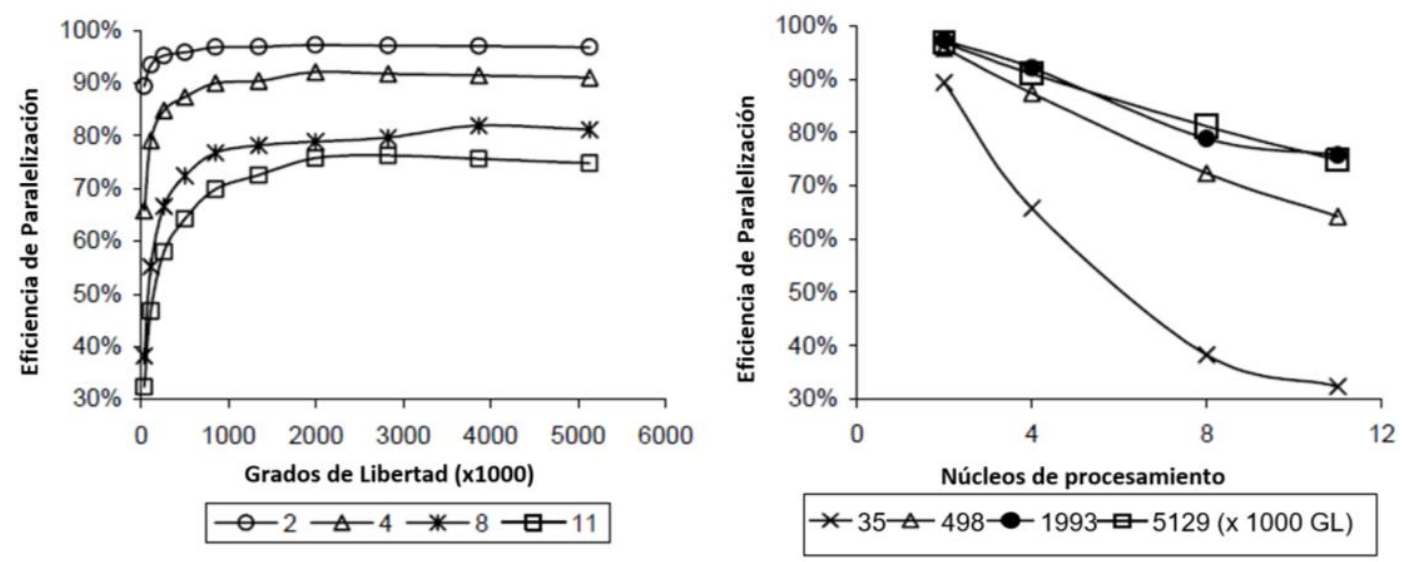

Figura 5.5: Eficiencia de la paralelización en función del tamaño de malla para diferente cantidad de núcleos (der.) y en función del número de núcleos para diferentes tamaños de malla (izq.).

De la misma manera se obtuvieron curvas similares para el "cluster" con la red de 100 Mbps pero no se muestran aquí. En su lugar, la figura 5.6 muestra la ganancia de "speed-up" al utilizar la red de 1 Gbps en comparación a la red de $100 \mathrm{Mbps}$. Se observa que a medida que se aumenta la cantidad de Grados de Libertad para un mismo número de hilos, la ventaja de tener una red más veloz se reduce, dado que es menor la cantidad relativa de datos transferidos por la red con respecto al tamaño del problema. También se observa que, para una dada cantidad de GL a mayor cantidad de núcleos de procesamiento mayor es el aprovechamiento de la velocidad de la red, dado que aumenta la cantidad de datos a transferir con la cantidad de hilos.

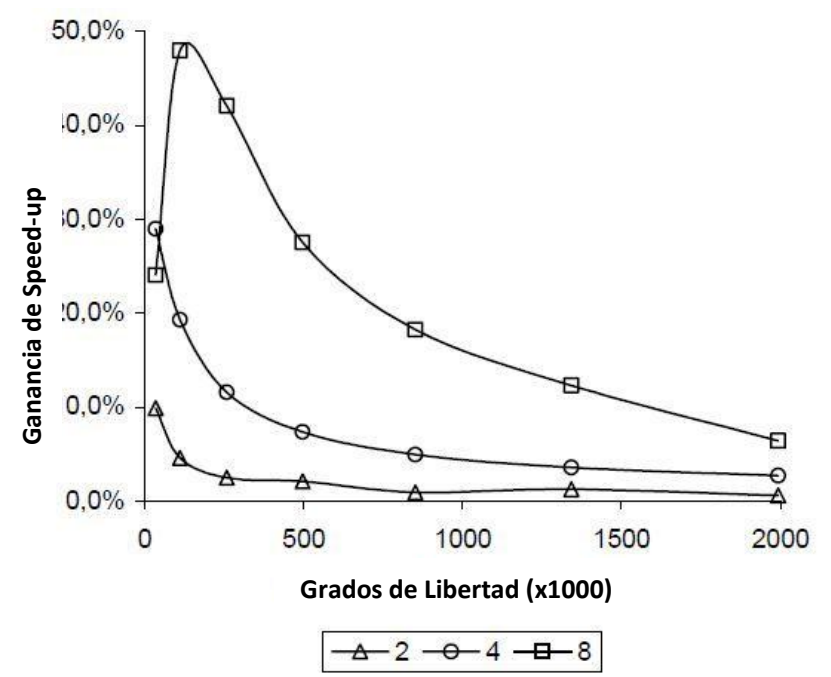

Figura 5.6: Ganancia de "speed-up" con el uso de la red de 1 Gbps comparada con la red de 100 Mbps en función del tamaño de malla. 


\section{CAPÍTULO 6}

\section{PROBLEMAS DE VERIFICACIÓN Y VALIDACIÓN.}

\subsection{FLUJO TRANSÓNICO LAMINAR EN TORNO A UN PERFIL AERODINÁMICO.}

El siguiente caso corresponde a un perfil aerodinámico NACA 0012 en régimen transónico: $\mathrm{M}=0.85, \mathrm{Re}=2000$. Este problema fue resuelto por varios autores ${ }^{[53],[54]}$ y por lo tanto se cuenta con resultados de referencia para los estudios comparativos. Aquí se presentan además los resultados obtenidos con la versión 17.1 de ANSYS/Fluent para el mismo problema. Todas las corridas realizadas para este caso son obtenidas con los esquemas TG, MTG y CBS con integración analítica completa para las matrices de masa y convectivas. Sólo se utilizan Jacobianos reducidos al centro del elemento para las matrices estabilizadoras y difusivas.

La geometría y otros parámetros importantes de este problema se muestran en la figura 6.1. La cuerda adimensionalizada del perfil tiene una longitud unitaria y el dominio se extiende cuarenta cuerdas hacia atrás desde el borde de fuga del perfil, veinte cuerdas hacia arriba, veinte cuerdas hacia abajo y el radio de la parte frontal es de veinte cuerdas también con su centro coincidente con el centro del radio del borde de ataque.

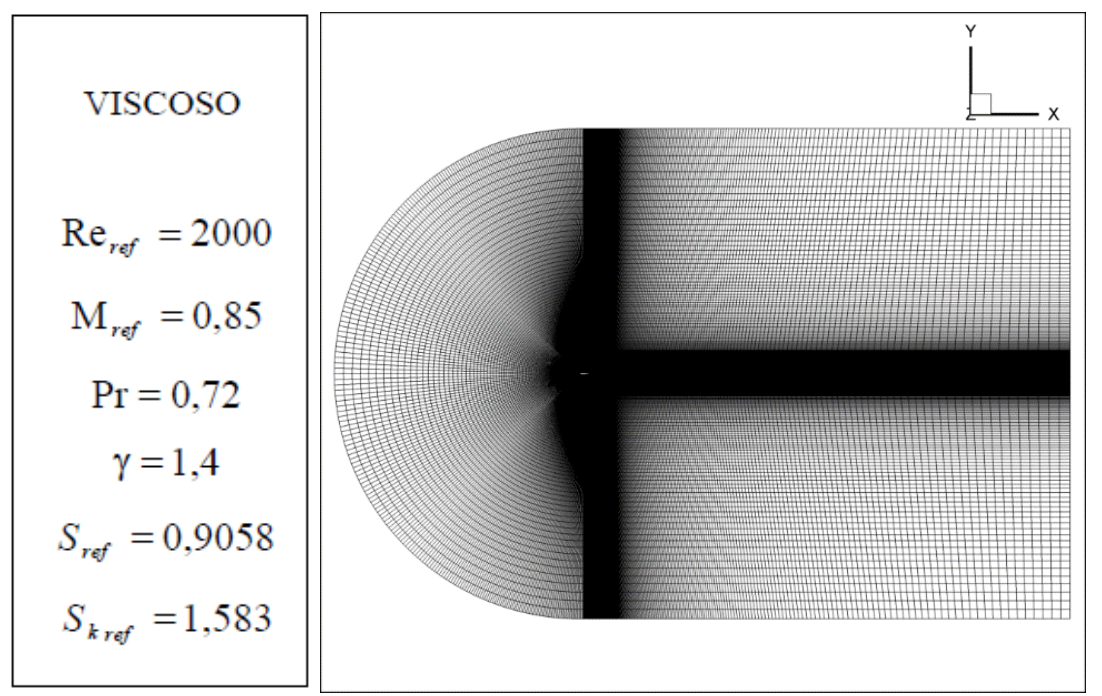

Figura 6.1 - Vista lateral del dominio y malla y características de flujo.

En las figuras 6.2 y 6.3 se muestran detalles de la vista lateral de la malla de elementos finitos, la cual cuenta con 386.560 elementos hexaédricos y 775.684 nodos. 
La misma malla se utiliza para las corridas con ANSYS/Fluent.

Considerando que la malla utilizada es tridimensional con un único elemento en la dirección $x_{3}$, se impone la condición $v_{3}=0,0$ en los contornos laterales de manera de evitar el flujo de masa a través de dichos contornos, conservando la característica bidimensional del problema. Es importante recordar aquí que, cuando se utiliza la formulación débil del FEM, las condiciones de contorno de Neumann prescriptas nulas (derivadas de variables, con respecto a la normal a los contornos, nulas) se satisfacen automáticamente si no se prescriben valores para las mismas variables en los correspondientes contornos.

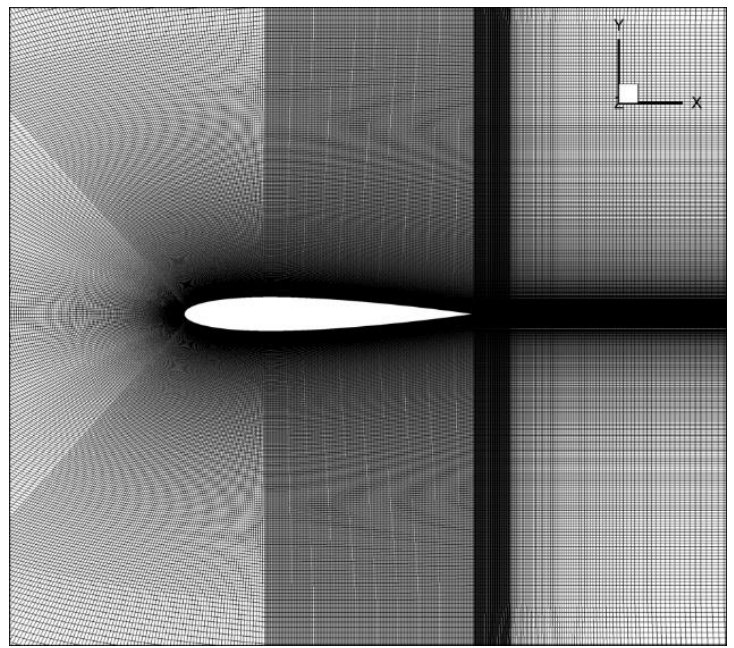

Figura 6.2 - Vista lateral de la malla: perfil completo.

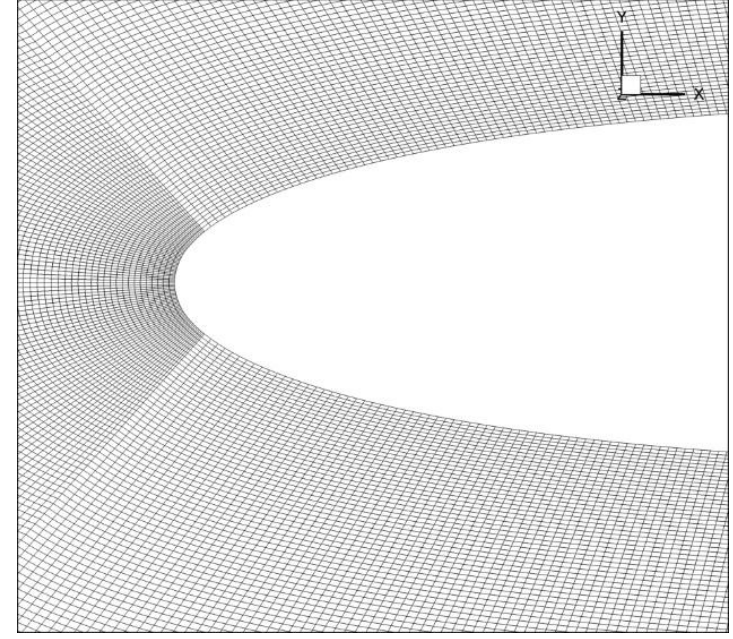

Figura 6.3: Vista lateral de la malla: borde de ataque.

Las condiciones de contorno en la entrada adimensionales son las siguientes:

$$
v_{1 \infty}=0,85 ; v_{2 \infty}=v_{3 \infty}=0,0 ; u_{\infty}=1,7857 ; \rho_{\infty}=1,0
$$

mientras que sobre los contornos sólidos (sobre el perfil) se aplica la condición de no deslizamiento, es decir:

$$
v_{1}=v_{2}=v_{3}=0,0
$$

en conjunto con la temperatura de estancamiento, la cual se especifica utilizando la energía interna específica, según la siguiente expresión:

$$
u_{s t g}=u_{\infty}\left(1+r \frac{\gamma-1}{2} M_{\infty}^{2}\right)=2,01535
$$


siendo $r=0,89$ el factor de recupero (un factor empírico introducido porque en realidad la recuperación de la energía no es perfecta), y no es necesario especificar condiciones en los contornos de salida, en los cuales sólo se definen los vectores $\left\{f_{j}\right\}$ y $\{q\}$.

Las condiciones iniciales vienen dadas por las siguientes expresiones:

$$
v_{1}{ }^{0}=0,85 ; v_{2}{ }^{0}=v_{3}{ }^{0}=0,00 ; u^{0}=1,7857 ; \rho^{0}=1,0 \text { у } p^{0}=0,71428
$$

Estos valores se definen en todos los nodos de la malla, a excepción de los nodos donde se prescribieron condiciones de contorno de Dirichlet.

Utilizando el factor de seguridad $\beta=0,2$, definido en (3.38) el paso de tiempo adimensional efectivo utilizado en este problema resultó $\Delta t=0,5^{*} 10^{-4}$. El coeficiente de viscosidad artificial, definido en (5.2), utilizado en este problema es $C_{A D}=0,0$.

El coeficiente de fricción superficial (skin friction) $c_{f}$ y el coeficiente de presión $c_{p}$ fueron calculados utilizando las siguientes expresiones

$$
c_{f}=\frac{\tau}{\frac{1}{2} \rho_{\infty}\left|v_{\infty}\right|^{2}} ; c_{p}=\frac{p-p_{\infty}}{\frac{1}{2} \rho_{\infty}\left|v_{\infty}\right|^{2}}
$$

donde $\tau$ y $p$ son las componentes tangencial y normal, respectivamente, de las fuerzas por unidad de área actuando en cada punto de la superficie de contorno sólido.

La figura 6.4 muestra los contornos de presión obtenidos con los esquemas MTG y CBS, que son prácticamente iguales. La figura 6.5 muestra los contornos de presión obtenidos con ANSYS/Fluent 17.1. Las imágenes muestran un excelente nivel de acuerdo.

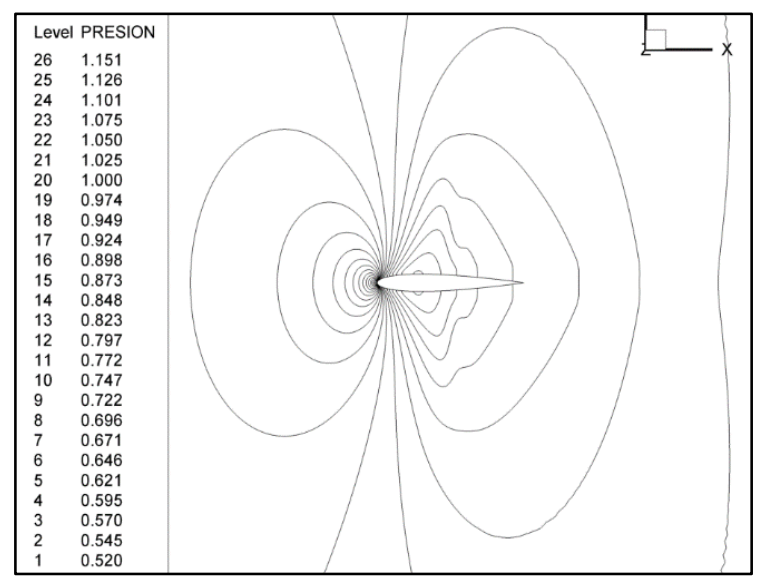

Figura 6.4 - Contornos de presión MTG y CBS.

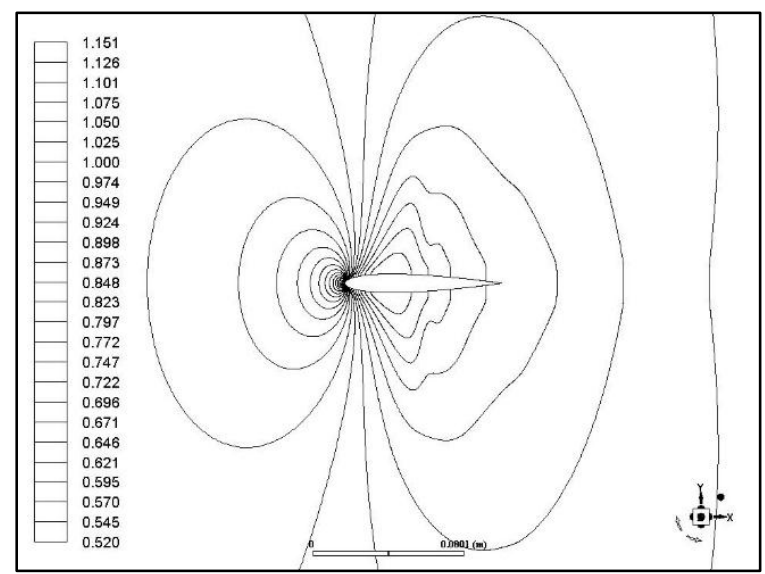

Figura 6.5: Contornos de presión ANSYS/Fluent 17.1.

La figura 6.6 muestra un detalle de los contornos de presión en el borde de ataque obtenidos con los esquemas MTG y CBS. La figura 6.7 muestra la misma imagen obtenida 
con el esquema TG2. Con el esquema TG2 pueden observarse oscilaciones de presión en zonas de baja velocidad, que no aparecen en los esquemas TG, CBS y MTG. Los esquemas CBS y MTG muestran prácticamente los mismos contornos de presión.

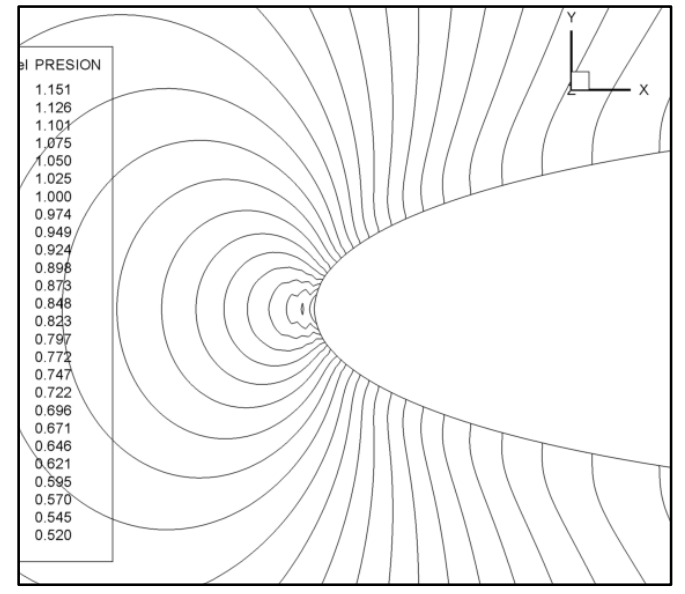

Figura 6.6 - Contornos de presión MTG y CBS.

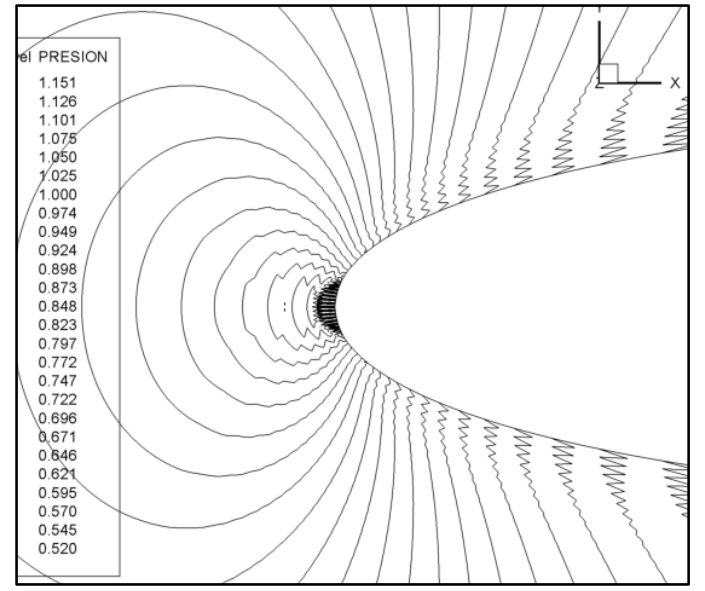

Figura 6.7: Contornos de presión TG2.

Todos estos resultados fueron obtenidos utilizando paso de tiempo interno local con todos los esquemas presentados (MTG, CBS, TG2 y TG).

En la figura 6.8 se muestra una imagen del coeficiente de fricción calculado con el método MTG con paso de tiempo interno, comparado con valores obtenidos numéricamente con la versión 17.1 de ANSYS/Fluent utilizando la misma malla. Se observa buena concordancia entre ambos resultados mostrados en la figura.

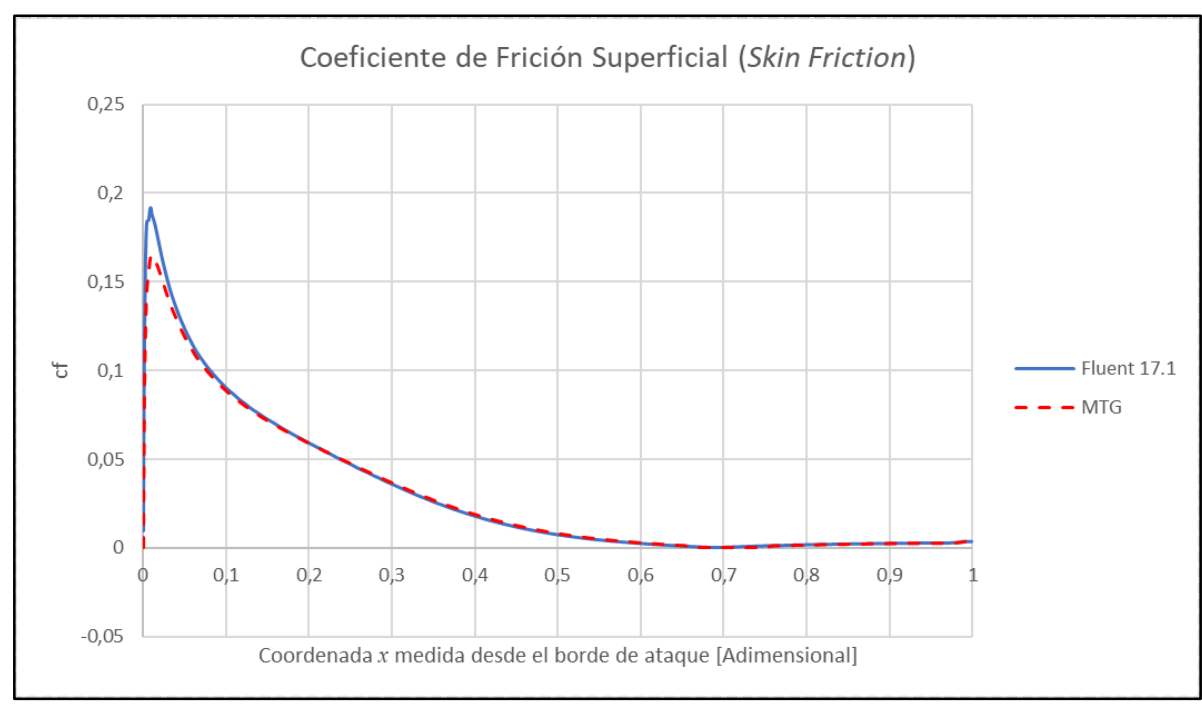

Figura 6.8 - Coeficiente de fricción sobre el perfil simétrico.

La figura 6.9 muestra los resultados numéricos obtenidos con ANSYS/Fluent $17.1 \mathrm{y}$ con el esquema MTG, para el coeficiente de presión sobre el perfil simétrico NACA 0012. 
Como puede verse, los resultados obtenidos para este coeficiente con el método MTG aplicando un paso de tiempo interior resultan muy próximos a los valores obtenidos con Fluent para la misma malla.

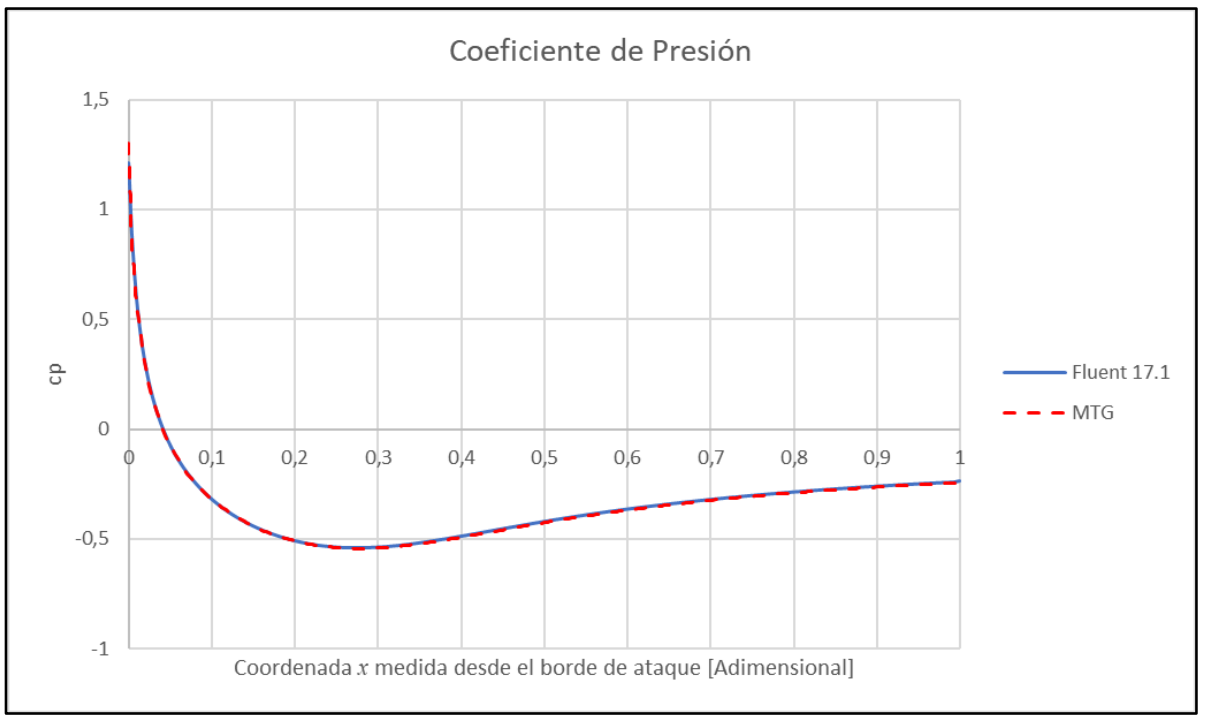

Figura 6.9 - Coeficiente de presión Fluent 17.1 y MTG $\left(\Delta \mathrm{t}_{\mathrm{int}}\right)$

En la figura 6.10 se muestra la convergencia de los esquemas considerados. El análisis de los contornos de presión y de los residuos de densidad arroja una clara determinación. Los métodos CBS y MTG presentan una mejora en la convergencia de densidad (y presión) con respecto al esquema TG2, cuando se los utilizan con un paso de tiempo interior local. De lo contrario la curva del residuo se coloca apenas por debajo de la curva obtenida con el esquema TG2. La convergencia del esquema TG fue testeada también (no aparece en la figura) y se coloca muy ligeramente por encima de las curvas del CBS y el MTG.

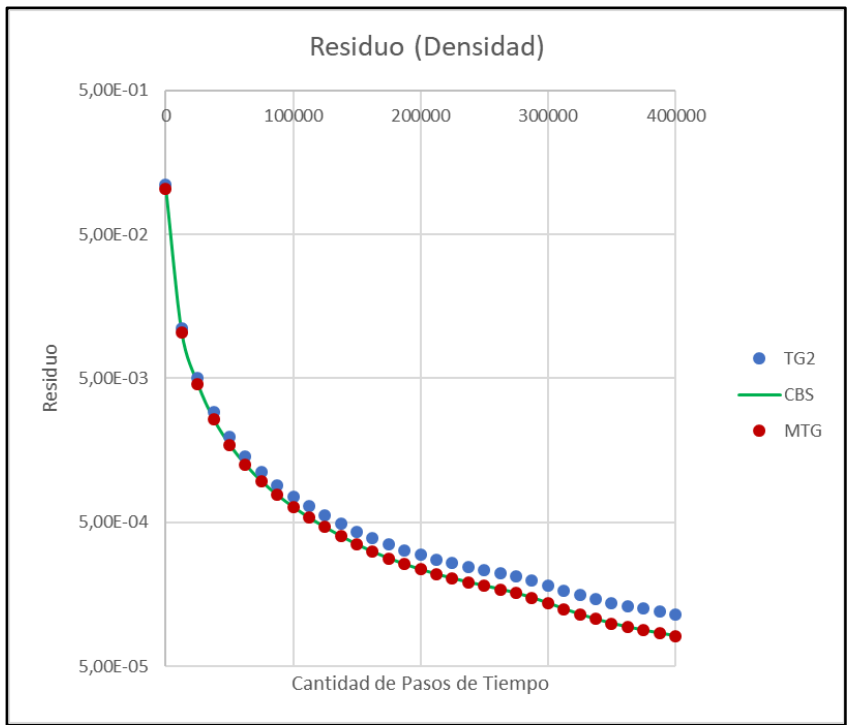

Figura 6.10 - Residuos mostrando convergencia de los diferentes algoritmos. 
También se observa que la utilización de un paso de tiempo interno con el método TG2 no ofrece ninguna ventaja comparativa con respecto al mismo esquema tomando un paso de tiempo único. En cambio, la performance del método MTG usando paso de tiempo interno local, es equivalente a la obtenida con el algoritmo CBS con paso de tiempo interno local.

Adicionalmente se hicieron corridas con el método CBS de un paso (CBS single-step) cuyos resultados no se muestran aquí, pero los mismos no difieren apreciablemente de los obtenidos con los esquemas TG, CBS o MTG.

Es importante, sin embargo, indicar los tiempos relativos para las diferentes corridas. Asumiendo un tiempo de referencia de valor 1 para el esquema más rápido, el CBS de un paso, en la siguiente tabla se muestran los tiempos relativos obtenidos corriendo este problema:

\begin{tabular}{|l|c|}
\hline Algoritmo: & Tiempo de cómputo relativo \\
\hline CBS de un paso & 1 \\
\hline TG & 1,14 \\
\hline TG2 & 1,01 \\
\hline MTG & 1,04 \\
\hline CBS & 1,33 \\
\hline
\end{tabular}

Tabla 6.1 - Tiempos de cómputo relativos.

Debe señalarse que los casos indicados en la tabla 1 corresponden todos a corridas del mismo problema en la misma computadora (Dell Precision 7610, 16 Cores, 64 Gb ram), y utilizando la misma cantidad de hilos en paralelo (16). Los tiempos que se comparan son tiempos de pared entre dos grabaciones de resultados con un mismo número de pasos de tiempo entre medio. Todos los algoritmos indicados aquí utilizan integración analítica exacta para las matrices de masa y convectivas, e integración analítica con reducción de las matrices Jacobianas al punto central para las matrices estabilizadoras y difusivas.

También debe indicarse que los códigos utilizados fueron desarrollados de la misma forma incluyendo únicamente aquellas modificaciones requeridas por los diferentes algoritmos. El primer código desarrollado correspondió al esquema TG y luego los restantes fueron obtenidos modificando este código original.

\subsection{FLUJO SUPERSÓNICO TURBULENTO SOBRE PLACA PLANA.}

El siguiente caso corresponde al flujo compresible turbulento sobre una placa plana en régimen supersónico: $\mathrm{M}=2, \mathrm{Re}=15^{*} 10^{6}$. 
Este problema se encuentra descripto en el repositorio web de la NASA para modelos de turbulencia (Turbulence Modeling Resource, Langley Research Center, NASA) ${ }^{[33]}$. Allí se muestran resultados para varios modelos de turbulencia y se lo compara con la resolución teórica semiempírica.

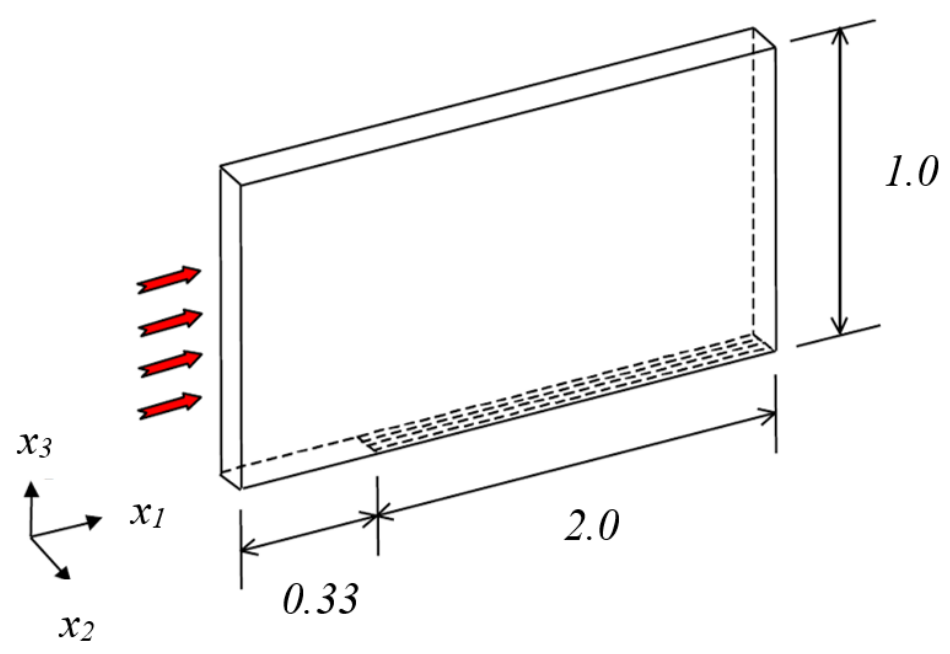

Figura 6.11 - Vista lateral y características de flujo.

La geometría y dirección de escurrimiento de este problema se muestran en la Figura 6.11. Considerando que la malla utilizada es tridimensional con un único elemento en la dirección $x_{2}$, se impone la condición $v_{2}=0,0$ en los contornos laterales de manera de evitar el flujo de masa a través de dichos contornos, conservando la característica bidimensional del problema.

En la Figura 6.12 se muestra la vista lateral de la malla de elementos finitos, la cual cuenta con 13.056 elementos hexaédricos y 26.578 nodos. La malla es exactamente la misma que se encuentra en el repositorio web de la NASA para modelos de turbulencia (3D 2x137x97/2x113 puntos sobre la placa plana).

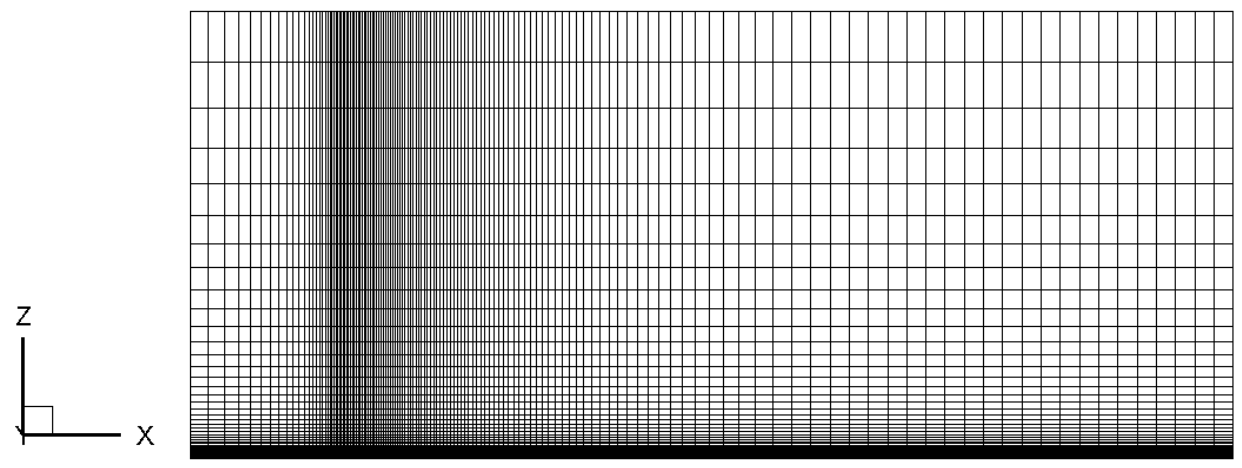

Figura 6.12 - Vista lateral de la malla. 
La malla tiene un refinamiento en proximidades de la pared tal que la estimación del y+ resulta próxima a 0,3 .

Las condiciones de contorno en la entrada son:

$$
v_{1 \infty}=2,0 ; v_{2 \infty}=v_{3 \infty}=0,0 ; u_{\infty}=1,7795 ; \rho_{\infty}=1,0 ; \tilde{v}_{\infty}=6,667 * 10^{-7}
$$

Sobre los contornos sólidos (sobre la placa) se aplica la condición de no deslizamiento

$$
v_{1}=v_{2}=v_{3}=0,0 ; \tilde{v}_{\infty}=0,0
$$

en conjunto con la temperatura de estancamiento, la cual se especifica utilizando la energía interna específica, según la siguiente expresión $(r=0,89)$

$$
u_{s t g}=u_{\infty}\left(1+r \frac{\gamma-1}{2} M_{\infty}^{2}\right)=3,0465
$$

y no es necesario especificar condiciones en los contornos de salida, en los cuales sólo se definen los vectores $\left\{f_{j}\right\}$ y $\{q\}$. Las condiciones iniciales vienen dadas por:

$$
v_{1}{ }^{0}=2,0 ; v_{2}{ }^{0}=v_{3}{ }^{0}=0,0 ; u^{0}=1,7795 ; \rho^{0}=1,0 ; p^{0}=0,7118 \text { y } \tilde{v}^{0}=6,667 * 10^{-7}
$$

Estos valores se definen en todos los nodos de la malla, a excepción de los nodos donde se prescribieron condiciones de contorno de Dirichlet.

Utilizando el factor de seguridad $\beta=0,2$, definido el paso de tiempo adimensional efectivo utilizado en este problema resultó $\Delta t=1,0^{*} 10^{-7}$. El coeficiente de viscosidad artificial utilizado en este problema es $C_{A D}=0,3$.

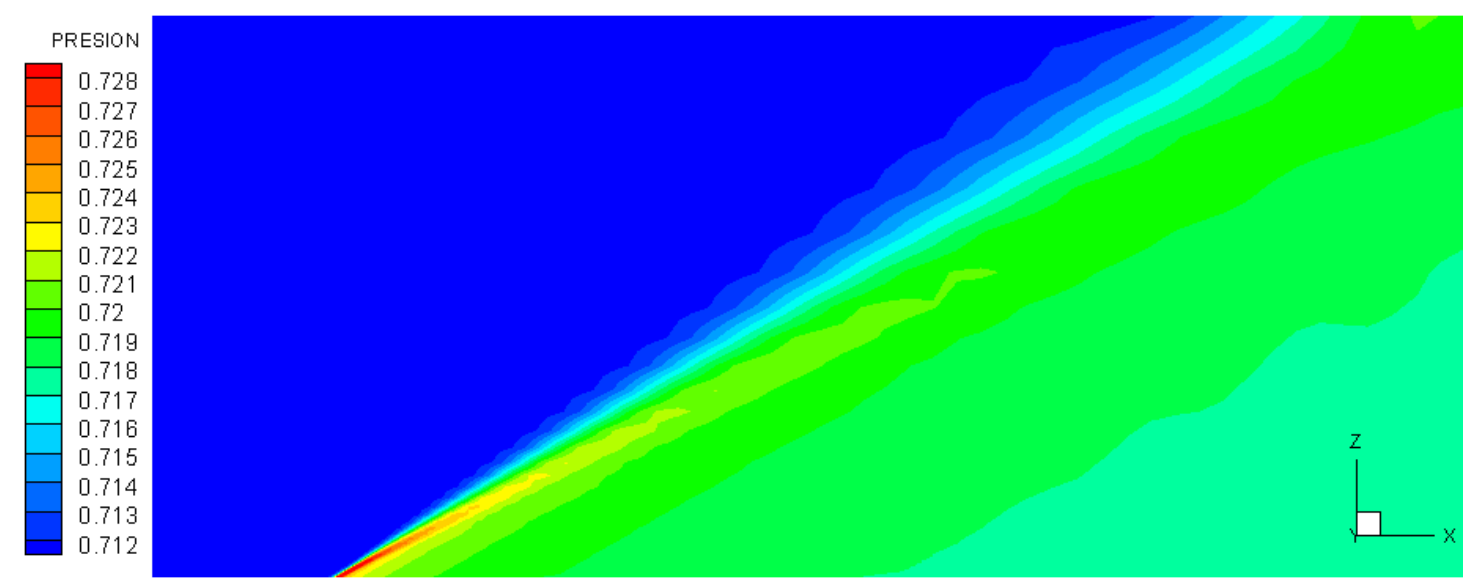

Figura 6.13 - Contornos de Presión (MTG)

La Figura 6.13 muestra los contornos de presión obtenidos en este trabajo. Los contornos obtenidos con los esquemas TG, TG2, MTG y CBS no muestran diferencias apreciables entre sí. Sin embargo, los investigadores que desarrollaron el esquema CBS [3],[17] 
señalan que el esquema CBS de un paso no es adecuado para altos números de Mach. Las simulaciones realizadas en este trabajo con dicho esquema para números de Mach elevados indican que la convergencia del CBS de un paso no es buena y pueden aparecer inestabilidades. Por lo tanto, se confirma aquí lo establecido por estos autores, y no se recomienda el uso del esquema CBS de un paso en estos casos.

En la Figura 6.14 se ven los contornos de presión obtenidos con ANSYS/Fluent 17.1 disponible en el GFC (Grupo de Fluidodinámica Computacional) de la UNLP (Universidad Nacional de La Plata). La malla utilizada para las simulaciones con Fluent es exactamente la misma que la mostrada en la Figura 6.12. Las escalas de las Figuras 6.13 y 6.14 han sido ajustadas para ser coincidentes. Los valores de presión adimensional son muy similares. En ambas imágenes las distribuciones de presión en el flujo muestran la perturbación producida por el borde de la placa propagándose como una onda de Mach.

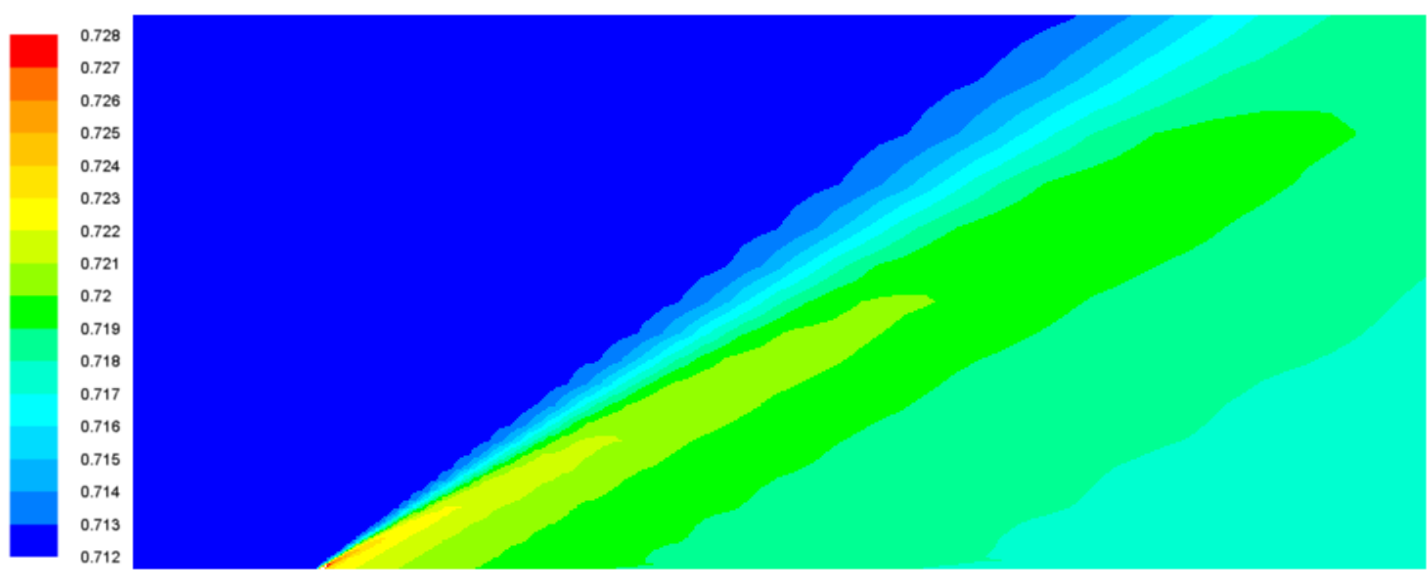

Figura 6.14- Contornos de Presión (ANSYS/Fluent 17.1)

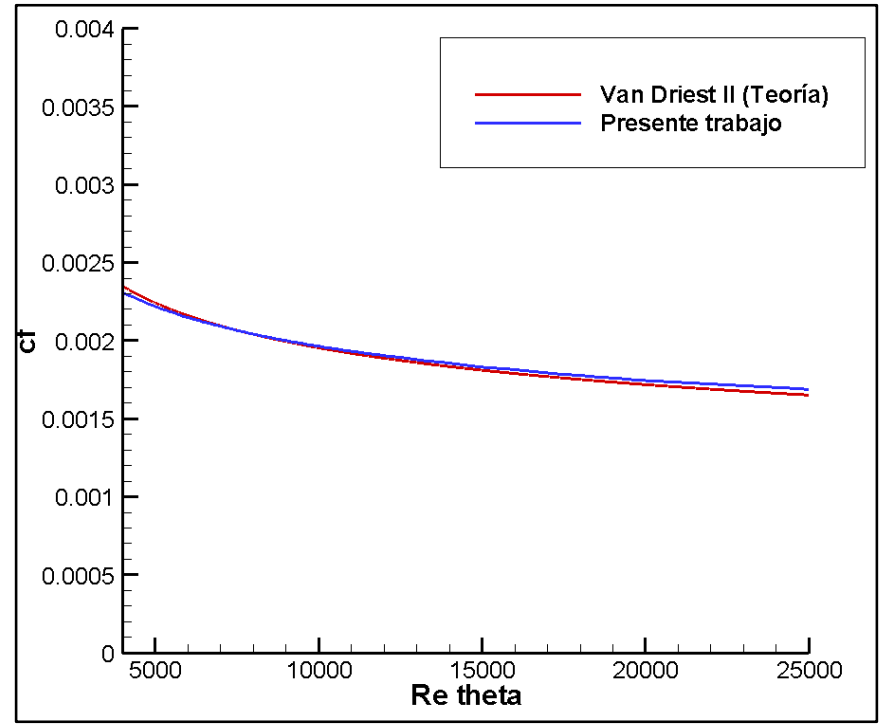

Figura 6.15 - Coeficiente de fricción sobre el perfil simétrico. 
En la Figura 6.15 se muestra una imagen del coeficiente de fricción, comparando con valores provistos por la correlación semiempírica de Van Driest II (ver repositorio web de la NASA para modelos de turbulencia ${ }^{[33]}$, que muestran la buena concordancia con los resultados mostrados en dicha referencia para este coeficiente. Nuevamente no hay diferencias apreciables entre los resultados obtenidos con los diferentes esquemas, TG, TG2, CBS y MTG. Todos estos esquemas resultan adecuados desde el punto de vista de la calidad de los resultados.

Por último, la Figura 6.16 muestra el perfil de velocidades adimensionalizado, correspondiente a la posición de la placa donde se verifica que $\operatorname{Re} \theta=10000$ ( $\theta$ es el espesor de cantidad de movimiento [33], [41] $)$. Como puede verse, los resultados obtenidos para en este trabajo resultan muy próximos a los valores que se muestran en el repositorio de la NASA y que corresponden a la correlación semiempírica de Van Driest I. Aunque no se muestran aquí, en este repositorio se muestran resultados obtenidos con otros códigos y las curvas casi se superponen con la curva obtenida en este trabajo.

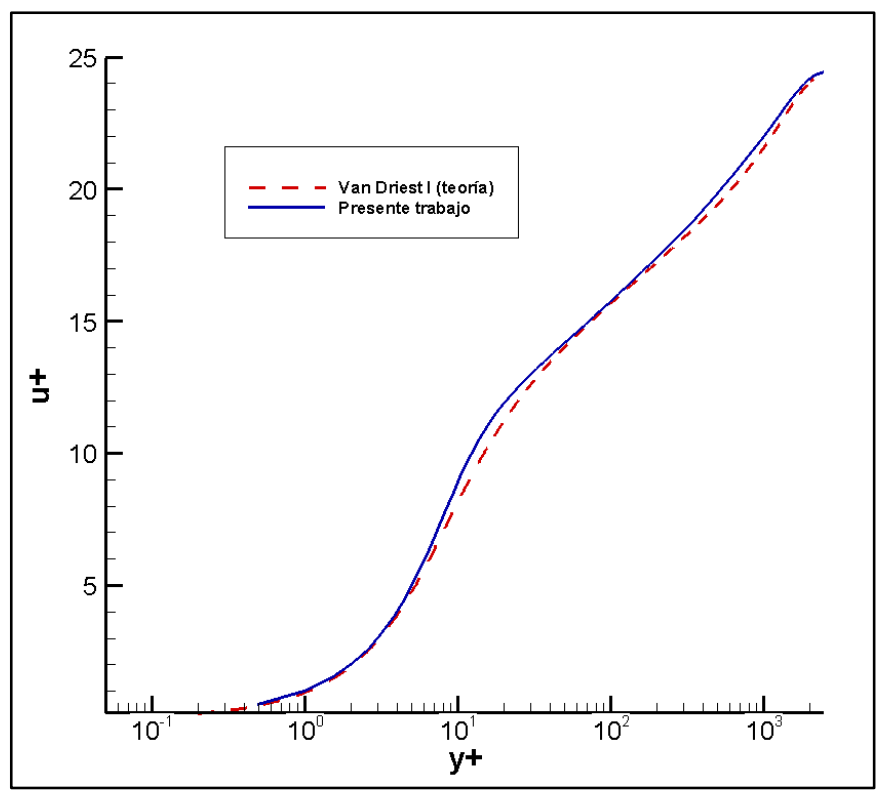

Figura 6.16 - Perfil de velocidad en $\operatorname{Re} \theta=10000$

\begin{tabular}{|l|c|}
\hline Algoritmo: & Tiempo de cómputo relativo \\
\hline CBS de un paso & No se recomienda \\
\hline TG & 1,16 \\
\hline TG2 & 1 \\
\hline MTG & 1,03 \\
\hline CBS & 1,3 \\
\hline
\end{tabular}

Tabla 6.2 - Tiempos de cómputo relativos. 
La convergencia entre los esquemas TG, TG2, MTG y CBS no difiere significativamente cuando se grafican los residuos, sin embargo, sí hay diferencias en los tiempos de cómputo relativos, como puede observarse en la tabla 6.2.

De esta forma se observa que, para números de Mach supersónicos, los cuatro algoritmos son adecuados desde el punto de vista de la convergencia, pero hay diferencias apreciables en cuanto a tiempo de cómputo de pared, siendo el esquema CBS el más oneroso en este sentido. Por su lado, el algoritmo CBS single-step de un paso no se recomienda para números de Mach no perturbados supersónicos.

\subsection{FLUJO HIPERSÓNICO "FRIO" TURBULENTO “ASWBLI": INTERACCIÓN ENTRE CAPA LÍMITE Y ONDA DE CHOQUE.}

El siguiente caso corresponde al flujo compresible turbulento sobre un obstáculo con forma de lanza en régimen hipersónico frío: $\mathrm{M}=7,11, \mathrm{Re} / \mathrm{L}=57.0601 / \mathrm{cm}$. Este problema se encuentra descripto en el repositorio web de la NASA para modelos de turbulencia (Turbulence Modeling Resource, Langley Research Center, NASA ${ }^{[33]}$ ). Allí se muestran resultados para varios modelos de turbulencia y se lo compara con datos experimentales. El propósito aquí es proporcionar un caso de validación para los modelos de turbulencia. A diferencia de la verificación, en la que busca establecer si un modelo se ha implementado correctamente por comparación con otros softwares o códigos, en la validación se comparan los resultados de CFD con datos experimentales, estableciendo la capacidad de un modelo para reproducir la física.

El estudio experimental realizado por Kussoy \& Horstman ${ }^{[55]}$ involucró un cilindro de cono / ojiva con un ángulo de conicidad de 20 grados. El borde delantero es un cono de 10 grados que hace la transición a través de un arco circular a un cilindro de área constante con un radio de $100 \mathrm{~mm}$. La conicidad, diseñada para producir un choque oblicuo y una zona de interacción entre la capa límite y la onda de choque, está ubicada 1390 mm aguas abajo del borde de ataque. Como se describe en Georgiadis et. al. (2015) ${ }^{[56]}$, el borde de ataque se puede excluir del cálculo de CFD, siempre que las condiciones de los límites de entrada se ajusten ligeramente (el número de Mach se cambia de 7,05 a 7,11); Esto es lo que se hace aquí. La pared (cilindro y cono) tiene una temperatura constante de $311 \mathrm{~K}$. Las características geométricas se describen en las figuras $6.17,6.18$ y 6.19 .

Si bien el problema es axialsimétrico, su resolución se realiza aquí con un 3D, tomando un cuarto de la geometría por consideraciones de simetría. 


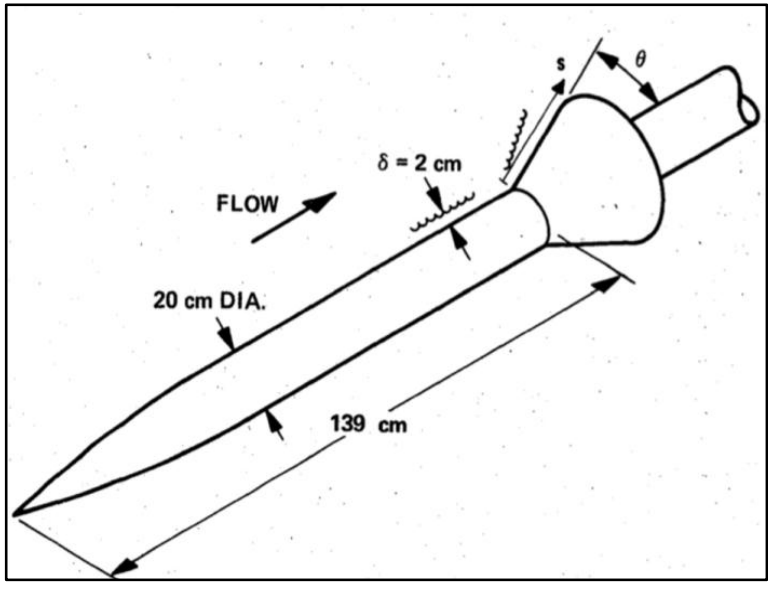

Figura 6.17 - Vista del cuerpo de lanza completo ${ }^{[33]}$.

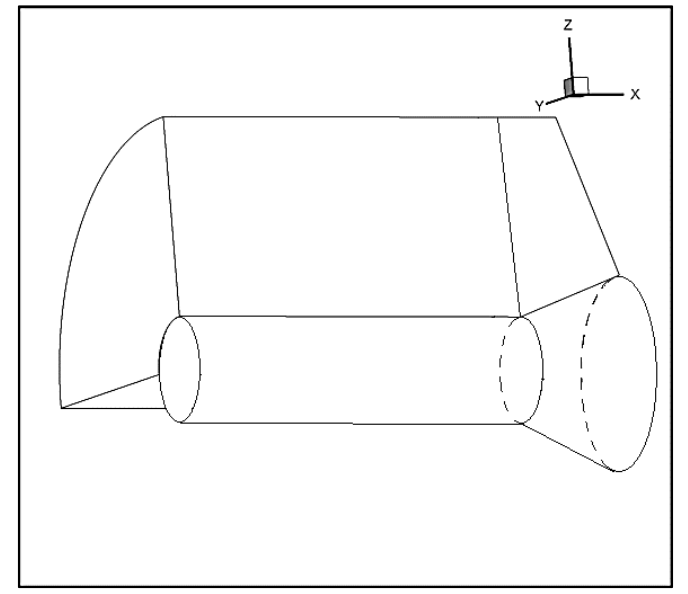

Figura 6.18 -Dominio y obstáculo cilíndrico cónico.

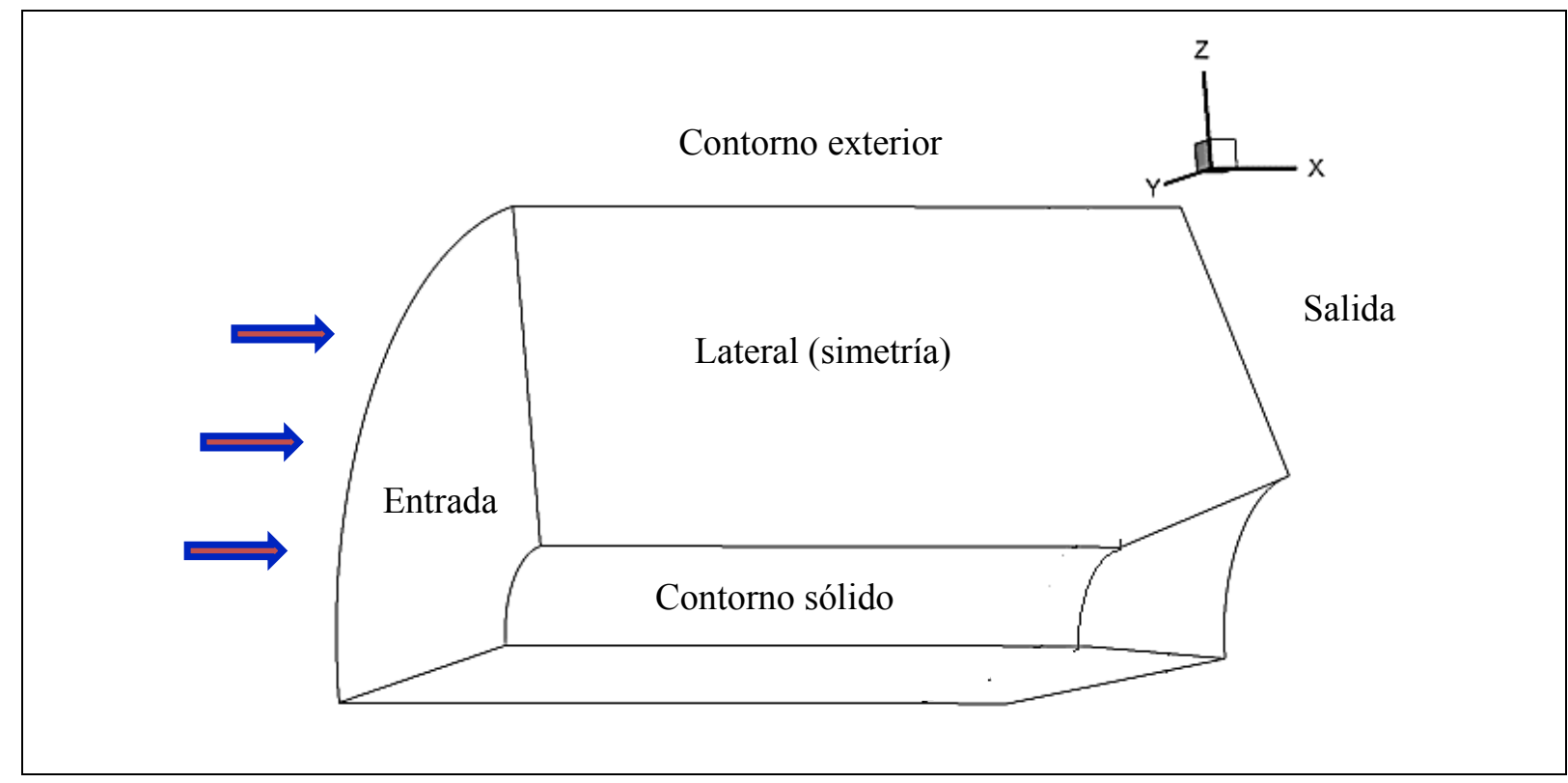

Figura 6.19 - Vista del dominio y contornos.

La geometría del dominio se muestra en las Figura 6.18 y 6.19. El problema fue adimensionalizado. En los dos contornos laterales se impone la condición de que la componente de velocidad perpendicular a dichos contornos sea nula. En la entrada las condiciones de contorno son las siguientes:

$$
v_{1 \infty}=7,11 ; v_{2 \infty}=v_{3 \infty}=0,0 ; u_{\infty}=1,7795 ; \rho_{\infty}=1,0 ; \tilde{v}_{\infty}=6,230284 * 10^{-4}
$$

Sobre los contornos sólidos se aplica la condición de no deslizamiento:

$$
v_{1}=v_{2}=v_{3}=0,0 ; \tilde{v}_{\infty}=0,0
$$


en conjunto con la temperatura en la pared, la cual es un es dato $(311 \mathrm{~K})$ y se especifica utilizando la energía interna específica:

$$
u_{w}=6,918
$$

y no es necesario especificar condiciones en los contornos de salida, en los cuales sólo se definen los vectores $\left\{f_{j}\right\}$ y $\{q\}$.

Las condiciones iniciales vienen dadas por:

$$
\begin{gathered}
v_{1}{ }^{0}=7,11 ; v_{2}{ }^{0}=v_{3}{ }^{0}=0,0 ; u^{0}=1,7795 ; \rho^{0}=1,0 \\
p^{0}=0,7118 \text { y } \tilde{v}^{0}=6,230284 * 10^{-4}
\end{gathered}
$$

Estos valores se definen en todos los nodos de la malla, a excepción de los nodos donde se prescribieron condiciones de contorno de Dirichlet.

En la Figura 6.20 se muestra la vista lateral de la malla de elementos finitos, la cual cuenta con 1.600.000 elementos hexaédricos y 1.642.361 nodos. La malla es exactamente la misma que se encuentra en el repositorio web de la NASA para modelos de turbulencia [(2-D) 2-zone 81x101 and 81x101] pero rotada para formar un dominio tridimensional correspondiente a un cuarto del espacio circundante al obstáculo.

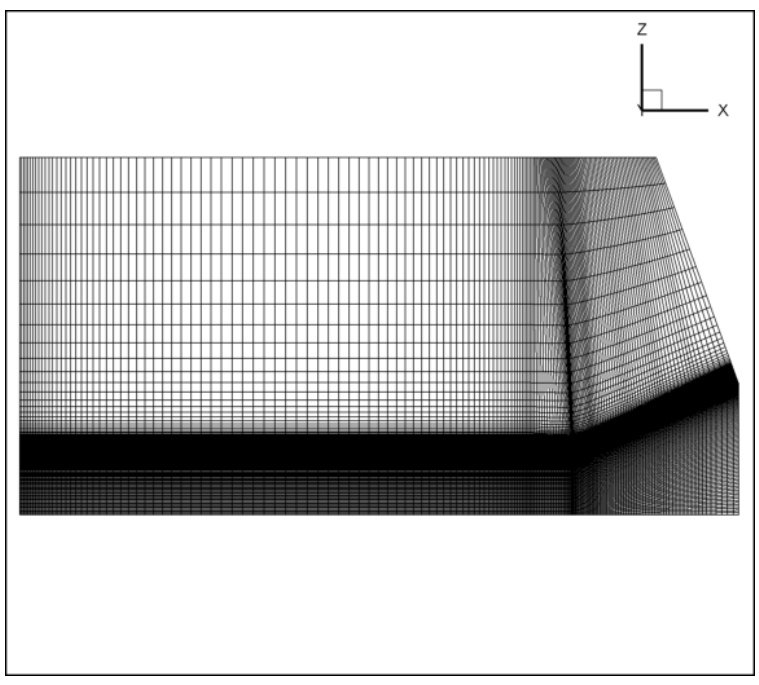

Figura 6.20-Malla de elementos finitos.

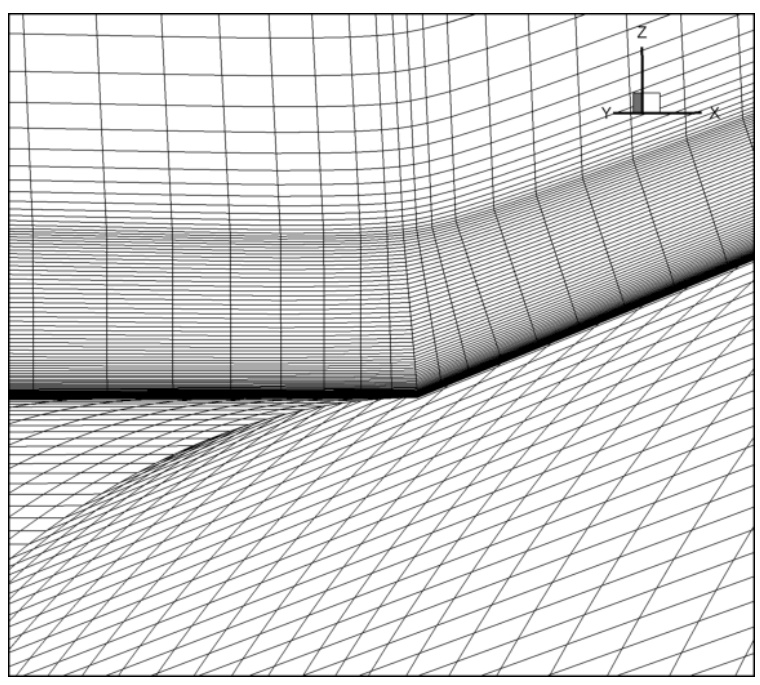

Figura 6.21 -Malla en la zona de inicio del cono.

La malla tiene un refinamiento en proximidades de la pared tal que la estimación del y+ resulta próximo a 0,5 .

Utilizando el factor de seguridad $\beta=0,2$, el paso de tiempo adimensional efectivo para este problema resultó $\Delta t=1,0^{*} 10^{-5}$. El coeficiente de viscosidad artificial utilizado es $C_{A D}=0,2$. 
En la figura 6.22 se comparan el perfil de velocidad experimental con los obtenidos con el programa WindUS de la NASA ${ }^{[33]}$ y con los obtenidos en este trabajo. La posición en la cual se obtuvieron estos datos corresponde al plano $x=-6$ (el origen de coordenadas $x=0$ se encuentra donde inicia el cono). Dado que no hay diferencias apreciables entre los resultados con TG, TG2, CBS y MTG, aquí se muestra únicamente una curva correspondiente al programa "Airflow" desarrollado en este trabajo utilizando el esquema MTG.

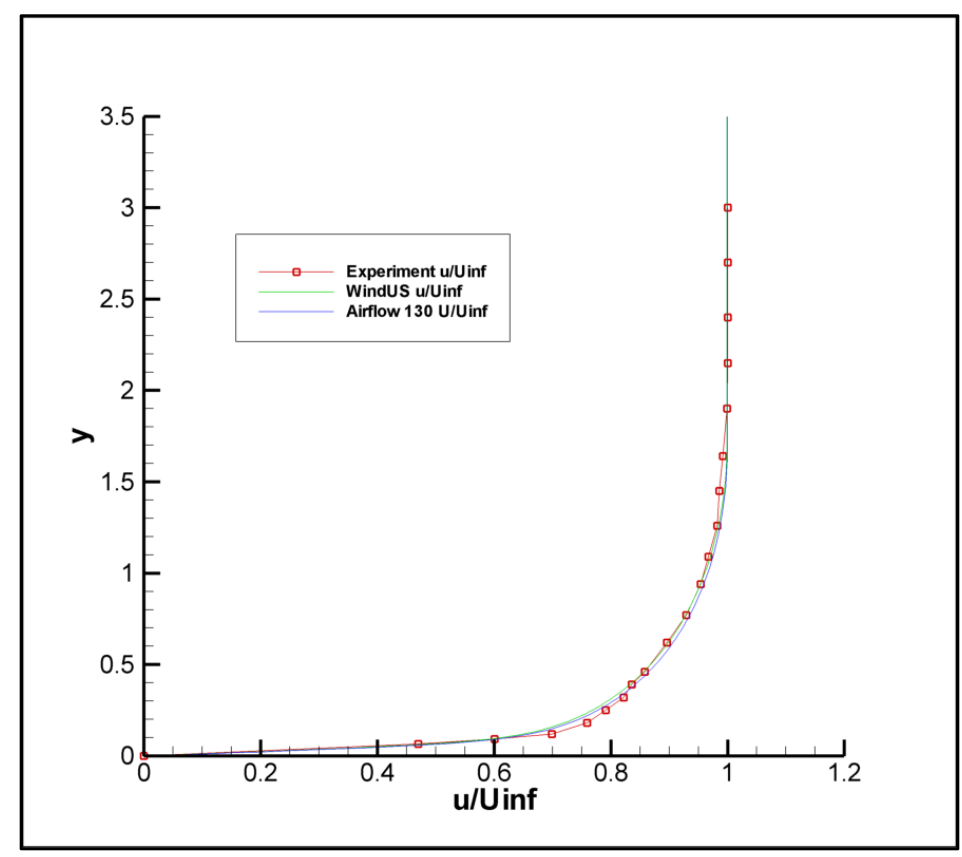

Figura 6.22 - Comparación de perfiles de velocidad.

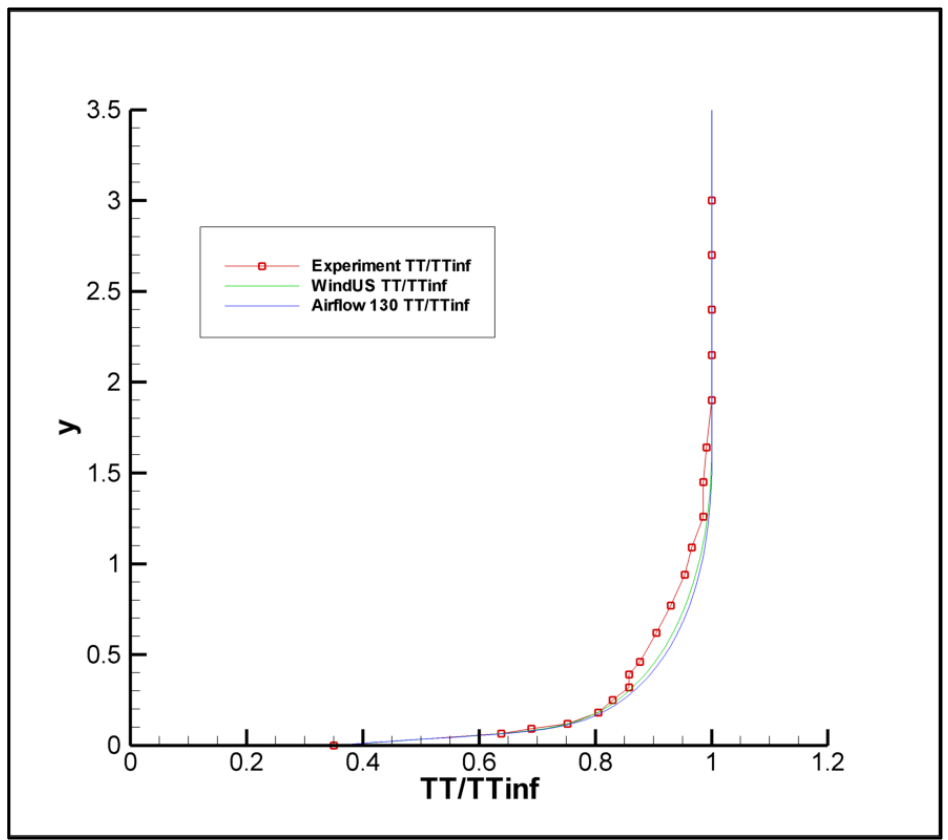

Figura 6.23 - Comparación de perfiles de temperatura. 
En la figura 6.23 se comparan los perfiles de temperatura para la misma ubicación. Ambas figuras muestran mucha proximidad entre los valores obtenidos con el programa "WindUS" (Ver Georgiadis et. al., ${ }^{[56]}$ ) y los códigos "Airflow" implementados en este trabajo. A su vez, ambos códigos muestran buen grado de proximidad con los datos experimentales obtenidos por Kussoy \& Horstman ${ }^{[55]}$.

La figura 6.24 muestra una comparación de la distribución de presiones a lo largo de la pared del obstáculo. La coordenada "S" es medida a lo largo de la superficie del sólido con su origen $\mathbf{S}=0$ correspondiendo al inicio del cono.

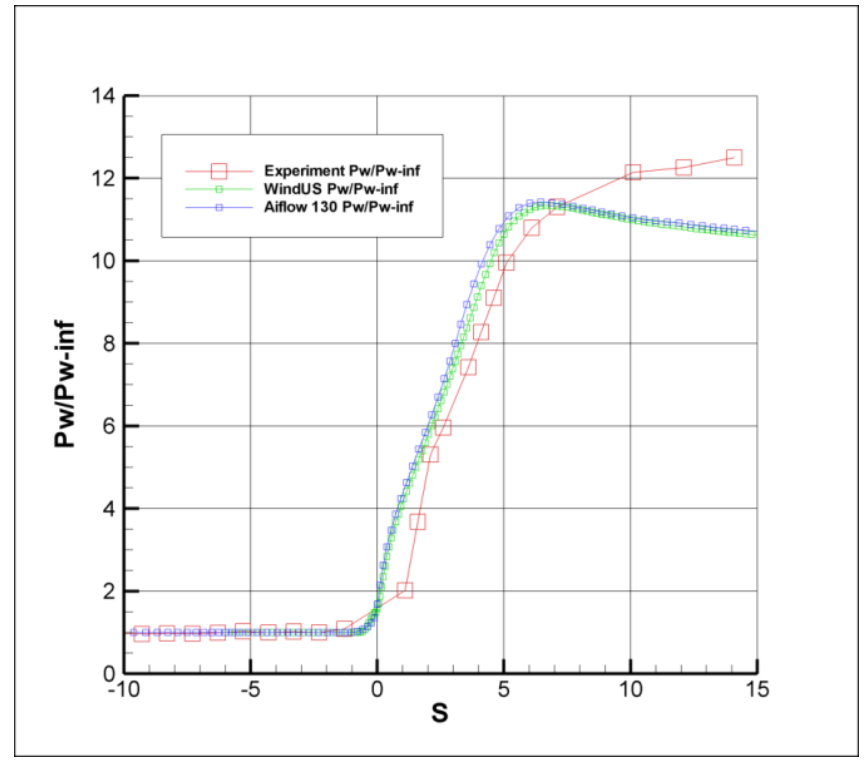

Figura 6.24 - Comparación de la distribución de presión sobre la pared de la lanza.

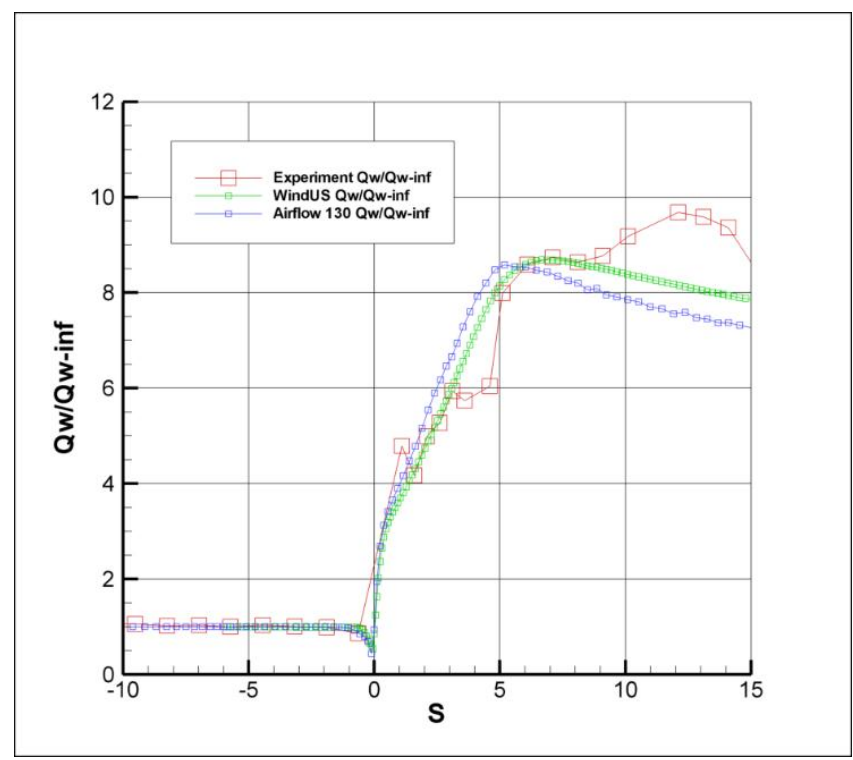

Figura 6.25 - Comparación de la transferencia de calor a través de la pared. 
Por último, la Figura 6.25 muestra una comparación de la transferencia de calor a través de la pared del obstáculo sólido. La definición de la transferencia de calor de pared viene dada por: $q_{w}=-K(d u / d y)_{w}$, dond $K$ es la conductividad térmica del fluido.

Se observa un buen grado de proximidad entre los códigos "Airflow" y "WindUs", con los datos experimentales. Las diferencias entre los resultados de ambos códigos y los datos experimentales pueden explicarse por el modelo de turbulencia adoptado (EVM-BoussinesqSpalart-Allmaras). Las simulaciones en las referencias [56] y [57] muestran el mismo resultado.

Al igual que en el caso anterior, la convergencia entre los esquemas TG, TG2, MTG y CBS no difiere significativamente cuando se grafican los residuos, sin embargo, sí hay diferencias en los tiempos de cómputo relativos, como puede observarse en la siguiente tabla:

\begin{tabular}{|l|c|}
\hline Algoritmo: & Tiempo de cómputo relativo \\
\hline CBS de un paso & No se recomienda \\
\hline TG & 1,18 \\
\hline TG2 & 1 \\
\hline MTG & 1,03 \\
\hline CBS & 1,32 \\
\hline
\end{tabular}

Tabla 6.3 - Tiempos de cómputo relativos.

De esta forma se observa que, para números de Mach hipersónicos, los tres algoritmos son adecuados desde el punto de vista de la convergencia, pero hay diferencias apreciables en cuanto a tiempo de cómputo de pared, siendo el esquema CBS el más oneroso en este sentido.

En cuanto al esquema CBS de un paso, este esquema fue corrido para este caso mostrando oscilaciones numéricas importantes en la zona inicio del cono, lo cual muestra que efectivamente este algoritmo no es recomendable para altos números de Mach.

Una observación importante, que aplica a los tiempos relativos del esquema TG mostrados en este capítulo para todos los ejemplos, es que el esquema TG utilizado aquí ha sido trabajado como se muestra en el Anexo I, lo cual ya implica en una considerable eliminación de términos sin cambiar en nada la esencia del método. Pero, si se aplicara en forma directa, usando la matriz $\boldsymbol{A}_{i}$ completa sin el retrabajo mostrado en el anexo I, seguramente el tiempo de computo sería aún mayor, comparable a los tiempos de cómputo del método CBS Split A. 


\section{CONCLUSIONES.}

Con respecto a la paralelización de los códigos implementados con los algoritmos TG, TG2, MTG, CBS, CBS single-step, todos ellos se mostraron adecuados para la paralelización, particularmente para las configuraciones de memoria distribuida. La división de tareas entre los procesadores lógicos es relativamente simple de realizar, así como la identificación de las variables involucradas en la comunicación entre los procesadores. Las características de los algoritmos empleados permiten que se adopte una estrategia de programa sin procesador maestro (Hostless program) resultando en una baja tasa de comunicación entre procesadores.

El balanceo de las cargas de trabajo empleado se mostró eficaz como para minimizar los efectos de no uniformidad del esfuerzo computacional y para permitir el ajuste de las cargas de trabajo asociadas a cada procesador cuando un indicador simple e imperfecto como la frecuencia del núcleo es utilizado como base para una división inicial de tareas.

El tratamiento de malla empleado para la minimización tanto de la cantidad de datos comunicados entre los procesadores como de la redundancia del esfuerzo computacional, presentan un buen desempeño en lo que respecta a la eficiencia de paralelización. Este se mostró adecuado cuando el número de núcleos de procesamiento no es muy grande en una configuración de memoria distribuida, cuando la latencia de comunicación en red es grande para esa misma configuración, o en configuraciones híbridas de memoria distribuida y memoria compartida.

La implementación utilizada fue totalmente basada en las capacidades presentadas por el lenguaje de programación FORTRAN 90 y la biblioteca MPI de comunicación. No fue utilizada ninguna característica especial del sistema operativo más allá del soporte a la comunicación en red. Esto probó ser una característica importante respecto de la portabilidad de estos códigos paralelos para ser utilizados en diferentes sistemas operativos diferentes.

Las implementaciones desarrolladas para este trabajo de tesis, así como en trabajos previos [58], [59], han mostrado que ambas estrategias de integración de matrices elementales aquí adoptadas son adecuadas para implementar en conjunto con los esquemas explícitos TG, TG2, CBS y MTG. Si bien en este trabajo no se evaluaron las implicancias de adoptar una 
integración analítica completa comparada con la integración analítica tomando los Jacobianos en el centro del elemento, sí se pudo mostrar que su implementación es factible.

La utilización de estos esquemas de integración resultó adecuada tanto en problemas no viscosos como viscosos laminares y turbulentos y para diferentes números de Mach. Queda como línea futura de investigación profundizar en las ventajas comparativas de estas técnicas de integración con respecto a la integración numérica usualmente utilizada en implementaciones de elementos finitos.

Con respecto a la calidad de convergencia del método CBS cuando se lo compara con el método TG, este trabajo confirma las afirmaciones de otros autores respecto de la mejora en la convergencia para bajos números de Mach $(\mathrm{M}<0.9)$ del método CBS respecto del TG, pero especialmente en su versión TG2. Pero, como demostraron estudios hechos en este trabajo, dicha mejora se hace más evidente cuando el método CBS se utiliza con pasos de tiempo locales (internos) para las matrices de estabilización. Estos trabajos también mostraron que cuando se utiliza el método de TG2, el uso de pasos de tiempo interiores no tiene mayor impacto.

La implementación del modelo de Spalart-Allmaras en el contexto del método de elementos finitos utilizando los esquemas TG, TG2, CBS y CBS single-step con integración analítica de las matrices elementales resultó exitosa. Aquí es importante mencionar que se utilizó la forma conservativa de la ecuación de transporte de Spalart-Allmaras, propuesta como alternativa para flujos compresibles por los mismos desarrolladores del modelo ${ }^{[40]}$. Queda para futuras investigaciones ver el comportamiento de este modelo en problemas de flujo desprendido y estelas de corte (shear layers).

Todo lo expresado aquí permite afirmar que el primer objetivo planteado en esta tesis se cumplió exitosamente. A su vez, el segundo objetivo planteaba la necesidad de realizar estudios comparativos de eficiencia de los diferentes métodos (TG, CBS y CBS single-step) en términos de tiempo requerido de cómputo. Las experiencias realizadas en este trabajo muestran que existe un costo de tiempo asociado a la necesidad de efectuar el Split característico del método CBS. Se propuso, entonces, un nuevo esquema híbrido denominado Taylor-Galerkin-Modificado (MTG).

Respecto de la convergencia, comparando los residuos, como se mencionó anteriormente, pudo observarse que el método CBS presenta mejor convergencia que el esquema TG2 y TG (en el caso del esquema TG2 es mucho más evidente, en el caso del esquema TG la diferencia es pequeña). Pero la mejor convergencia se obtiene usando CBS 
con $\Delta t_{\text {in }}$ local. En particular, el método TG2 presenta un comportamiento oscilatorio en las zonas de baja velocidad que no aparece con el método CBS. El nuevo método aquí propuesto, MTG, al igual que el CBS no presenta las oscilaciones de presión. La convergencia del método MTG se mostró, así, similar al método CBS. Utilizando un paso de tiempo interno local con el método TG2, no se observa ninguna mejora en términos de convergencia de densidad y de oscilación de presión. Sin embargo, utilizando un paso de tiempo interno local, el método MTG presenta una convergencia comparable con la del método CBS.

Los tiempos de cómputo muestran que los esquemas TG2 y MTG son los únicos que se aproximan a los valores obtenidos con el CBS single-step. Tanto el esquema TG original como el CBS Split $A$ muestran costos adicionales importantes en términos de tiempo de cómputo.

Si bien el método CBS no requiere de un proceso iterativo como sí lo requieren TG, TG2 y MTG en la práctica estos términos iterativos sólo se calculan en pocos pasos de tiempo, por lo cual los métodos TG2 y MTG resultan más eficientes que el método CBS, ya que no requieren hacer el "split" que implica calcular una variable auxiliar de cantidad de movimiento para luego utilizarla para calcular la densidad y, posteriormente, actualizar los valores de cantidad de movimiento. De esta forma, con la convergencia mejorada que presenta el método MTG, se tiene un esquema más eficiente que el esquema CBS y que presenta una convergencia comparable a este último esquema para todos los rangos de números de Mach. La alternativa del esquema CBS de un paso (CBS single-step), si bien resulta eficiente y adecuada para regímenes transónicos, no es recomendable para altos números de Mach, en régimen supersónico o hipersónico. El esquema TG2 tampoco resulta recomendable porque muestra oscilaciones en zonas de bajas velocidades.

El trabajo demuestra que la modificación propuesta con el esquema MTG es la mejor opción cuando se consideran la convergencia y los tiempos de cómputo para un amplio abanico de números de Mach para flujos compresibles laminares y turbulentos. Lo antedicho puede resumirse en la siguiente tabla:

\begin{tabular}{|l|c|c|c|}
\hline Algoritmo: & Convergencia a $M<1$ & Convergencia a $M>1$ & $\begin{array}{c}\text { Titempo de cómputo } \\
\text { relativo }\end{array}$ \\
\hline CBS de un paso & Buena & Mala & Óptimo \\
\hline TG & Regular/Buena & Buena & Oneroso \\
\hline TG2 & Regular/Mala & Buena & Casi óptimo \\
\hline MTG & Buena & Buena & Casi óptimo \\
\hline CBS & Buena & Buena & Muy oneroso \\
\hline
\end{tabular}

Tabla 6.4 - Comparación de esquemas numéricos. 
Como fuera mencionado anteriormente, los tiempos relativos del esquema TG mostrados en este trabajo, corresponden al esquema TG que ha sido trabajado como se muestra en el Anexo I, lo cual ya implica en una considerable eliminación de términos sin cambiar en nada la esencia del método. Pero, si se aplicara en forma directa, usando la matriz $\boldsymbol{A}_{\mathrm{i}}$ completa sin el retrabajo mostrado en el anexo I, seguramente el tiempo de computo del esquema TG sería aún mayor, comparable a los tiempos de cómputo del método CBS Split A.

Todo lo mencionado arriba se realizó, como es evidente, con códigos propios que están disponibles tanto para el GFC del departamento de ingeniería aeronáutica de la UNLP como para el CEDI de la UTN Facultad Regional Haedo. En particular, este último grupo tiene como misión derramar los desarrollos y resultados de sus actividades al resto de la comunidad académica de la Facultad Regional Haedo, por lo cual el tercer objetivo planteado en esta tesis, de compartir los códigos y conocimientos con la comunidad académica, se encuentra parcialmente alcanzado. Sin embargo, para que este objetivo sea completamente alcanzado, aún deben desarrollarse tareas, algunas de las cuales ya se encuentran planteadas en el Proyecto de Investigación (PID) homologado en 2019 por el rectorado de la UTN. Una de estas tareas consiste en desarrollar una plataforma de pre y pos-procesamiento a partir de software comercial o de libre disponibilidad, de manera de hacer más sencilla la utilización de estos programas por parte de alumnos de grado y posgrado.

De modo que los objetivos planteados en esta tesis, la evaluación del desempeño de los algoritmos de Taylor-Galerkin y CBS en elementos hexaédricos con integración analítica de matrices elementales, la mejora en su eficiencia a través de las modificaciones planteadas en el método MTG, y el desarrollo de códigos propios, ya paralelizados, para su implementación y difusión en el ambiente académico, se consideran alcanzados de forma satisfactoria. 


\section{ANEXO I}

\section{INTRODUCCIÓN}

Según fue visto en el capítulo 3, el esquema de Taylor Galerkin (TG) viene dado por la expresión (3.16), es decir:

$$
\begin{aligned}
\Delta \boldsymbol{U}_{I+1}^{n+1}= & \Delta t\left[-\frac{\partial \boldsymbol{F}_{i}{ }^{n}}{\partial x_{i}}-\frac{\partial \boldsymbol{G}_{i}{ }^{n}}{\partial x_{i}}-\boldsymbol{Q}^{n}+\frac{\Delta t}{2} \frac{\partial}{\partial x_{k}}\left(\boldsymbol{A}_{k}{ }^{n} \frac{\partial \boldsymbol{F}_{i}{ }^{n}}{\partial x_{i}}+\boldsymbol{Q}^{n}\right)\right] \\
& +\frac{\Delta t}{2}\left[-\frac{\partial \Delta \boldsymbol{F}_{i_{I}}^{n+1}}{\partial x_{i}}-\frac{\partial \Delta \boldsymbol{G}_{i_{I}}^{n+1}}{\partial x_{i}}-\Delta \boldsymbol{Q}_{I}^{n+1}+\frac{\Delta t}{2} \frac{\partial}{\partial x_{k}}\left(\boldsymbol{A}_{k}{ }^{n} \frac{\partial \Delta \boldsymbol{F}_{i_{I}}{ }^{n+1}}{\partial x_{i}}\right)\right]
\end{aligned}
$$

donde se utiliza el subíndice " $P$ " para indicar iteración anterior e " $I+1$ ” iteración actual.

El desarrollo de los términos que contienen a la matriz Jacobiana de Euler puede ser realizado en forma directa, como indica la expresión (3.16), pero también pueden ser retrabajados de manera de reducir considerablemente la cantidad de términos y matrices a considerar. Este retrabajo no modifica en nada la esencia del método y conduce a las expresiones (3.33) a (5.37) vistas en el capítulo 3, las cuales son equivalentes a las que se llegaría por medio del uso directo de la matriz $\boldsymbol{A}_{k}$ completa.

\section{MATRIZ JACOBIANA DE EULER $\boldsymbol{A}_{i}$}

Las matrices Jacobianas de Euler vienen dadas por la siguiente expresión:

$$
\boldsymbol{A}_{i}=\left[\begin{array}{cccccc}
0 & \delta_{i 1} & \delta_{i 1} & \delta_{i 1} & 0 & 0 \\
0,5 V^{2} \bar{\gamma} \delta_{i 1}-v_{1} v_{i} & (1-\bar{\gamma}) v_{1} \delta_{i 1}+v_{i} & v_{1} \delta_{i 2}-v_{2} \bar{\gamma} \delta_{i 1} & v_{1} \delta_{i 3}-v_{3} \bar{\gamma} \delta_{i 1} & \bar{\gamma} \delta_{i 1} & 0 \\
0,5 V^{2} \bar{\gamma} \delta_{i 2}-v_{2} v_{i} & v_{2} \delta_{i 1}-v_{1} \bar{\gamma} \delta_{i 2} & (1-\bar{\gamma}) v_{2} \delta_{i 2}+v_{i} & v_{2} \delta_{i 3}-v_{3} \bar{\gamma} \delta_{i 2} & \bar{\gamma} \delta_{i 2} & 0 \\
0,5 V^{2} \bar{\gamma} \delta_{i 3}-v_{3} v_{i} & v_{3} \delta_{i 1}-v_{1} \bar{\gamma} \delta_{i 3} & v_{3} \delta_{i 2}-v_{2} \bar{\gamma} \delta_{i 3} & (1-\bar{\gamma}) v_{3} \delta_{i 3}+v_{i} & \bar{\gamma} \delta_{i 3} & 0 \\
\left(V^{2} \bar{\gamma}-\gamma e\right) v_{i} & \bar{\varepsilon} \delta_{i 1}-v_{1} \bar{\gamma} v_{i} & \bar{\varepsilon} \delta_{i 2}-v_{2} \bar{\gamma} v_{i} & \bar{\varepsilon} \delta_{i 3}-v_{3} \bar{\gamma} v_{i} & \gamma v_{i} & 0 \\
-\tilde{v} v_{i} & \tilde{v} \delta_{i 1} & \tilde{v} \delta_{i 2} & \tilde{v} \delta_{i 3} & 0 & v_{i}
\end{array}\right]
$$

en donde se tiene que:

$$
\bar{\gamma}=\gamma-1 ; \bar{\varepsilon}=\gamma e-0,5 V^{2} \bar{\gamma} ; e=u+0,5 V^{2} ; V^{2}=v_{j} v_{j} ; p=\rho u \bar{\gamma}
$$

Como se ve, son tres matrices $\boldsymbol{A}_{i}$ para $i=1,2,3$. Por ejemplo, si $i=1$ se tiene:

$$
\boldsymbol{A}_{1}=\left[\begin{array}{cccccc}
0 & 1 & 0 & 0 & 0 & 0 \\
0,5 \bar{\gamma} V^{2}-v_{1}^{2} & (3-\gamma) v_{1} & v_{2} \bar{\gamma} & v_{3} \bar{\gamma} & \bar{\gamma} & 0 \\
-v_{2} v_{1} & v_{2} & v_{1} & 0 & 0 & 0 \\
-v_{3} v_{1} & v_{3} & 0 & v_{1} & 0 & 0 \\
\left(V^{2} \bar{\gamma}-\gamma e\right) v_{1} & \bar{\varepsilon}-v_{1}^{2} \bar{\gamma} & -v_{2} \bar{\gamma} v_{1} & -v_{3} \bar{\gamma} v_{1} & \gamma v_{1} & 0 \\
-\tilde{v} v_{1} & \tilde{v} & 0 & 0 & 0 & v_{1}
\end{array}\right]
$$


y, análogamente, se pueden expresar las matrices $\boldsymbol{A}_{2}$ y $\boldsymbol{A}_{3}$.

\section{EXPANSIÓN DE LOS TÉRMINOS DE ESTABILIZACIÓN}

Al aplicar el método de elementos finitos a los términos de estabilización que contienen a las matrices Jacobianas de Euler en (AI.1), es decir, cuando se desarrolla la siguiente expresión:

$$
\frac{\Delta t}{2} \frac{\partial}{\partial x_{k}}\left(\boldsymbol{A}_{k}{ }^{n} \frac{\partial \boldsymbol{F}_{i}{ }^{n}}{\partial x_{i}}\right)
$$

según fuera indicado en la sección 3.1.2 de este trabajo, se obtiene la siguiente expresión matricial para dichos términos:

$$
\left[\boldsymbol{A}_{i}{ }^{n}\right]\left\{\boldsymbol{F}_{i}\right\}^{n}
$$

en donde:

$$
\left\{\boldsymbol{F}_{i}\right\}^{n}=\left\{\begin{array}{c}
\left\{\rho v_{i}\right\} \\
\left\{\rho v_{1} v_{i}+p \delta_{i 1}\right\} \\
\left\{\rho v_{2} v_{i}+p \delta_{i 2}\right\} \\
\left\{\rho v_{3} v_{i}+p \delta_{i 3}\right\} \\
\left\{(\rho e+p) v_{i}\right\} \\
\left\{\rho \tilde{v} v_{i}\right\}
\end{array}\right\}^{n}
$$

y las matrices $\left[\boldsymbol{A}_{i}{ }^{n}\right]$ pueden entenderse como tres matrices de $6 \times 6$ donde cada uno de sus elementos es, a su vez una matriz de $8 \times 8$. Estas matrices quedan definidas por la siguiente expresión:

$$
\boldsymbol{A}_{i_{M N}}=\frac{\Delta t}{2} \int_{\Omega_{E}} \boldsymbol{A}_{k}{ }^{n} \frac{\partial \Phi_{M}}{\partial x_{k}} \frac{\partial \Phi_{N}}{\partial x_{i}} d \Omega
$$

en donde que $\boldsymbol{A}_{k}{ }^{n}$ es de 6 x6, $i, k=1,2,3$ y $M, N=1,2, \ldots, 8$.

Considerando los valores promedio de elemento de las matrices $\boldsymbol{A}_{k}$ de la expresión (AI.2), estos valores pueden salir de las integrales de la misma forma que los valores de $v_{k}$ en la expresión (4.46) para las matrices $\left[C_{i}\right] \mathrm{y}$, entonces, las matrices $\left[\boldsymbol{A}_{i}\right]$ quedan definidas por la siguiente expresión: 


$$
\left[\boldsymbol{A}_{i}{ }^{n}\right]=\left[\begin{array}{llllll}
\left(A_{k_{11}}\right)_{E}\left[\alpha_{k i}\right] & \left(A_{k_{12}}\right)_{E}\left[\alpha_{k i}\right] & \left(A_{k_{13}}\right)_{E}\left[\alpha_{k i}\right] & \left(A_{k_{14}}\right)_{E}\left[\alpha_{k i}\right] & \left(A_{k_{15}}\right)_{E}\left[\alpha_{k i}\right] & \left(A_{k_{16}}\right)_{E}\left[\alpha_{k i}\right] \\
\left(A_{k_{21}}\right)_{E}\left[\alpha_{k i}\right] & \left(A_{k_{22}}\right)_{E}\left[\alpha_{k i}\right] & \left(A_{k_{23}}\right)_{E}\left[\alpha_{k i}\right] & \left(A_{k_{24}}\right)_{E}\left[\alpha_{k i}\right] & \left(A_{k_{25}}\right)_{E}\left[\alpha_{k i}\right] & \left(A_{k_{26}}\right)_{E}\left[\alpha_{k i}\right] \\
\left(A_{k_{31}}\right)_{E}\left[\alpha_{k i}\right] & \left(A_{k_{32}}\right)_{E}\left[\alpha_{k i}\right] & \left(A_{k_{33}}\right)_{E}\left[\alpha_{k i}\right] & \left(A_{k_{34}}\right)_{E}\left[\alpha_{k i}\right] & \left(A_{k_{35}}\right)_{E}\left[\alpha_{k i}\right] & \left(A_{k_{36}}\right)_{E}\left[\alpha_{k i}\right] \\
\left(A_{k_{41}}\right)_{E}\left[\alpha_{k i}\right] & \left(A_{k_{42}}\right)_{E}\left[\alpha_{k i}\right] & \left(A_{k_{43}}\right)_{E}\left[\alpha_{k i}\right] & \left(A_{k_{44}}\right)_{E}\left[\alpha_{k i}\right] & \left(A_{k_{45}}\right)_{E}\left[\alpha_{k i}\right] & \left(A_{k_{46}}\right)_{E}\left[\alpha_{k i}\right] \\
\left(A_{k_{51}}\right)_{E}\left[\alpha_{k i}\right] & \left(A_{k_{52}}\right)_{E}\left[\alpha_{k i}\right] & \left(A_{k_{53}}\right)_{E}\left[\alpha_{k i}\right] & \left(A_{k_{54}}\right)_{E}\left[\alpha_{k i}\right] & \left(A_{k_{55}}\right)_{E}\left[\alpha_{k i}\right] & \left(A_{k_{56}}\right)_{E}\left[\alpha_{k i}\right] \\
\left(A_{k_{61}}\right)_{E}\left[\alpha_{k i}\right] & \left(A_{k_{62}}\right)_{E}\left[\alpha_{k i}\right] & \left(A_{k_{63}}\right)_{E}\left[\alpha_{k i}\right] & \left(A_{k_{64}}\right)_{E}\left[\alpha_{k i}\right] & \left(A_{k_{65}}\right)_{E}\left[\alpha_{k i}\right] & \left(A_{k_{66}}\right)_{E}\left[\alpha_{k i}\right]
\end{array}\right]
$$

en donde las matrices $\left[\alpha_{k i}\right]$ son de 8x8 y son las mismas que se tenían en las expresiones (3.37) y (4.27), es decir:

$$
\begin{aligned}
\alpha_{i j_{M N}}=\frac{\Delta t_{i n}}{2} & \int_{\Omega_{E}} \frac{\partial \Phi_{M}}{\partial x_{i}} \frac{\partial \Phi_{N}}{\partial x_{j}} d \Omega \\
= & \frac{\Delta t_{i n}}{2} \int_{-1}^{1} \int_{-1}^{1} \int_{-1}^{1}\left(\frac{\partial \Phi_{M}}{\partial \xi_{k}} J(0)_{i k}^{-1}\right)\left(\frac{\partial \Phi_{N}}{\partial \xi_{h}} J(0)_{j h}^{-1}\right)|J(0)| d \xi_{1} d \xi_{2} d \xi_{3}
\end{aligned}
$$

Se procede, entonces, a expandir la expresión (AI.6) teniendo en cuenta que la misma contiene a los términos estabilizadores no iterativos de las cinco ecuaciones de conservación (masa, $3 \mathrm{x}$ cantidad de movimiento y energía) y la ecuación de transporte de SA. Además, debe observarse que los términos estabilizadores iterativos toman la misma forma, por lo cual no requieren un desarrollo específico.

\section{Ecuación de continuidad:}

Desarrollando el producto $\left[\boldsymbol{A}_{i}{ }^{n}\right]\left\{\boldsymbol{F}_{i}\right\}^{n}$ para la primera ecuación y considerando los valores de la primera fila de cada una de las tres matrices $\boldsymbol{A}_{k}{ }^{n}$, se tiene la siguiente expresión para los términos estabilizadores de la ecuación de conservación de la masa:

$$
\begin{gathered}
\delta_{k 1}\left[\alpha_{k i}\right]\left\{F_{i 1}^{C M}\right\}^{n}+\delta_{k 2}\left[\alpha_{k i}\right]\left\{F_{i 2}^{C M}\right\}^{n}+\delta_{k 3}\left[\alpha_{k i}\right]\left\{F_{i 3}^{C M}\right\}^{n}= \\
{\left[\alpha_{1 i}\right]\left\{F_{i 1}^{C M}\right\}^{n}+\left[\alpha_{2 i}\right]\left\{F_{i 2}^{C M}\right\}^{n}+\left[\alpha_{3 i}\right]\left\{F_{i 3}^{C M}\right\}^{n}}
\end{gathered}
$$

en donde las $\left[\alpha_{k i}\right]$ son matrices simétricas. La expresión anterior ya puede utilizarse en las ecuaciones, pero un retrabajo adicional permite reutilizar matrices ya calculadas. En efecto, considerando que:

$$
\left\{F_{i j}^{C M}\right\}^{n}=\left\{\rho v_{i} v_{j}+\delta_{i j} p\right\}^{n} ;\left\{F_{i}^{M}\right\}^{n}=\left\{\rho v_{i}\right\}^{n}
$$

puede sacarse $v_{j}$ del vector $\left\{F_{i j}^{C M}\right\}^{n}$ como un promedio de elemento, con lo cual resulta la siguiente expresión para los términos estabilizadores: 


$$
\begin{gathered}
{\left[\alpha_{1 i}\right]\left\{F_{i 1}^{C M}\right\}^{n}+\left[\alpha_{2 i}\right]\left\{F_{i 2}^{C M}\right\}^{n}+\left[\alpha_{3 i}\right]\left\{F_{i 3}^{C M}\right\}^{n}=} \\
\left(v_{1}\right)_{E}\left[\alpha_{1 i}\right]\left\{F_{i}^{M}\right\}^{n}+\left(v_{2}\right)_{E}\left[\alpha_{2 i}\right]\left\{F_{i}^{M}\right\}^{n}+\left(v_{3}\right)_{E}\left[\alpha_{3 i}\right]\left\{F_{i}^{M}\right\}^{n}+ \\
{\left[\alpha_{1 i}\right]\left\{\delta_{i 1} p\right\}^{n}+\left[\alpha_{2 i}\right]\left\{\delta_{i 2} p\right\}^{n}+\left[\alpha_{3 i}\right]\left\{\delta_{i 3} p\right\}^{n}}
\end{gathered}
$$

y, finalmente, teniendo en cuenta la expresión (4.48) los términos estabilizadores en (AI.13) para la ecuación de continuidad pueden calcularse de la siguiente manera:

$$
\begin{gathered}
{\left[C_{i}\right]\left\{F_{i}^{M}\right\}^{n}+\left(\left[\alpha_{11}\right]+\left[\alpha_{22}\right]+\left[\alpha_{33}\right]\right)\{p\}^{n}=} \\
{\left[C_{i}\right]\left\{F_{i}^{M}\right\}^{n}+\left[\alpha_{i i}\right]\{p\}^{n}=} \\
{\left[C_{i}\right]\left\{F_{i}^{M}\right\}^{n}+[P]\{p\}^{n}}
\end{gathered}
$$

Ecuaciones de conservación de cantidad de movimiento

Desarrollando el producto $\left[\boldsymbol{A}_{i}{ }^{n}\right]\left\{\boldsymbol{F}_{i}\right\}^{n}$ para la segunda ecuación, la que corresponde a $\rho v_{1}$, y considerando los valores de la segunda fila de cada una de las tres matrices $\boldsymbol{A}_{k}{ }^{n}$, se tiene la siguiente expresión para los términos estabilizadores de la ecuación de la primera componente de la conservación de cantidad de movimiento:

$$
\begin{gathered}
\left(\delta_{k 1} 0,5 \bar{\gamma} V^{2}-v_{1} v_{k}\right)_{E}\left[\alpha_{k i}\right]\left\{F_{i}^{M}\right\}^{n}+\left((1-\bar{\gamma}) v_{1} \delta_{k 1}+v_{k}\right)_{E}\left[\alpha_{k i}\right]\left\{F_{i 1}^{C M}\right\}^{n}+ \\
\left(v_{1} \delta_{k 2}-v_{2} \bar{\gamma} \delta_{k 1}\right)_{E}\left[\alpha_{k i}\right]\left\{F_{i 2}^{C M}\right\}^{n}+\left(v_{1} \delta_{k 3}-v_{3} \bar{\gamma} \delta_{k 1}\right)_{E}\left[\alpha_{k i}\right]\left\{F_{i 3}^{C M}\right\}^{n}+ \\
\left(\delta_{k 1} \bar{\gamma}\right)\left[\alpha_{k i}\right]\left\{F_{i}^{E}\right\}^{n}
\end{gathered}
$$

Resolviendo las sumas con las Deltas de Kronecker, teniendo en cuenta la expresión (4.48) y distribuyendo algunos productos, la anterior expresión puede llevarse a la siguiente forma:

$$
\begin{gathered}
0,5 \bar{\gamma} V^{2}\left[\alpha_{1 i}\right]\left\{F_{i}^{M}\right\}^{n}-\left(v_{1}\right)_{E}\left[C_{i}\right]\left\{F_{i}^{M}\right\}^{n}+\left[C_{i}\right]\left\{F_{i 1}^{C M}\right\}^{n}+ \\
\left(v_{1}\left[\alpha_{1 i}\right]-\bar{\gamma} v_{1}\left[\alpha_{1 i}\right]\right)_{E}\left\{F_{i 1}^{C M}\right\}^{n}+\left(v_{1}\left[\alpha_{2 i}\right]-\bar{\gamma} v_{2}\left[\alpha_{1 i}\right]\right)_{E}\left\{F_{i 2}^{C M}\right\}^{n}+ \\
\left(v_{1}\left[\alpha_{3 i}\right]-\bar{\gamma} v_{3}\left[\alpha_{1 i}\right]\right)_{E}\left\{F_{i 3}^{C M}\right\}^{n}+\bar{\gamma}\left[\alpha_{1 i}\right]\left\{F_{i}^{E}\right\}^{n}
\end{gathered}
$$

Considerando que:

$$
\left\{F_{i j}^{C M}\right\}^{n}=\left\{\rho v_{i} v_{j}+\delta_{i j} p\right\}^{n}=\left\{F_{j i}^{C M}\right\}^{n} ;\left\{F_{i}^{M}\right\}^{n}=\left\{\rho v_{i}\right\}^{n}
$$

puede sacarse $v_{j}$ del vector $\left\{F_{j i}^{C M}\right\}^{n}$ como un promedio de elemento en los términos que contienen a $\bar{\gamma}$, con lo cual, simplificando con el primer término, resulta la siguiente expresión para los términos estabilizadores: 


$$
\begin{gathered}
-\left[0,5 \bar{\gamma} V^{2}\left[\alpha_{1 i}\right]\left\{F_{i}^{M}\right\}^{n}+\bar{\gamma}\left[C_{1}\right]\{p\}^{n}\right]-\left(v_{1}\right)_{E}\left[C_{i}\right]\left\{F_{i}^{M}\right\}^{n}+\left[C_{i}\right]\left\{F_{i 1}^{C M}\right\}^{n}+ \\
\left(v_{1}\left[\alpha_{1 i}\right]\right)_{E}\left\{F_{i 1}^{C M}\right\}^{n}+\left(v_{1}\left[\alpha_{2 i}\right]\right)_{E}\left\{F_{i 2}^{C M}\right\}^{n}+\left(v_{1}\left[\alpha_{3 i}\right]\right)_{E}\left\{F_{i 3}^{C M}\right\}^{n}+ \\
\bar{\gamma}\left[\alpha_{1 i}\right]\left\{F_{i}^{E}\right\}^{n}
\end{gathered}
$$

Ahora haciendo la misma operación que la indicada en (AI.17) con los tres términos afectados por el factor $\left(v_{1}\left[\alpha_{k i}\right]\right)_{E}$ se puede expresar:

$$
\begin{gathered}
-0,5 \bar{\gamma} V^{2}\left[\alpha_{1 i}\right]\left\{F_{i}^{M}\right\}^{n}-\bar{\gamma}\left[C_{1}\right]\{p\}^{n}-\left(v_{1}\right)_{E}\left[C_{i}\right]\left\{F_{i}^{M}\right\}^{n}+\left[C_{i}\right]\left\{F_{i 1}^{C M}\right\}^{n}- \\
\left(v_{1}\right)_{E}\left[C_{i}\right]\left\{F_{i}^{M}\right\}^{n}+\left(v_{1}\right)_{E}\left[\alpha_{i i}\right]\{p\}^{n}+ \\
\bar{\gamma}\left[\alpha_{1 i}\right]\left\{F_{i}^{E}\right\}^{n}
\end{gathered}
$$

en donde, obviamente, hay dos términos que se pueden simplificar y, además, el término que contiene a $\left\{F_{i}^{E}\right\}^{n}$ también se puede expandir considerando que:

$$
\left\{F_{i}^{E}\right\}^{n}=\left\{\rho v_{i} e+p v_{i}\right\}^{n} ;\left\{F_{i}^{M}\right\}^{n}=\left\{\rho v_{i}\right\}^{n}
$$

con lo cual la expresión (AI.20) resulta:

$$
\begin{gathered}
-0,5 \bar{\gamma} V^{2}\left[\alpha_{1 i}\right]\left\{F_{i}^{M}\right\}^{n}-\bar{\gamma}\left[C_{1}\right]\{p\}^{n}+\left[C_{i}\right]\left\{F_{i 1}^{C M}\right\}^{n}+ \\
\left(v_{1}\right)_{E}\left[\alpha_{i i}\right]\{p\}^{n}+ \\
(\bar{\gamma} e)_{E}\left[\alpha_{1 i}\right]\left\{F_{i}^{M}\right\}^{n}+\bar{\gamma}\left[C_{1}\right]\{p\}^{n}
\end{gathered}
$$

Entonces, luego de simplificar los términos en la expresión anterior, la expresión (AI.21) resulta:

$$
\left[C_{i}\right]\left\{F_{i 1}^{C M}\right\}^{n}-0,5 \bar{\gamma} V^{2}\left[\alpha_{1 i}\right]\left\{F_{i}^{M}\right\}^{n}+\left(v_{1}\right)_{E}\left[\alpha_{i i}\right]\{p\}^{n}+(\bar{\gamma} e)_{E}\left[\alpha_{1 i}\right]\left\{F_{i}^{M}\right\}^{n}
$$

Pero esta última expresión aún admite un retrabajo adicional para llevarla a una forma que minimice aún más los requerimientos de tiempo de cómputo. En efecto, teniendo en cuenta que:

$$
e=u+0.5 V^{2}=\frac{p}{\rho \bar{\gamma}}+0,5 V^{2}
$$

los dos términos que contienen al vector $\left\{F_{i}^{M}\right\}^{n}$ en la expresión (AI.22) se pueden reducir y, finalmente, resulta:

$$
\left[C_{i}\right]\left\{F_{i 1}^{C M}\right\}^{n}+\left(v_{1}\right)_{E}\left[\alpha_{i i}\right]\{p\}^{n}+(p)_{E}\left[\alpha_{1 i}\right]\left\{v_{i}\right\}^{n}
$$

Por último, desarrollando el producto $\left[\boldsymbol{A}_{i}{ }^{n}\right]\left\{\boldsymbol{F}_{i}\right\}^{n}$ para las ecuaciones tercera y cuarta, las que corresponden a $\rho v_{2}$ y $\rho v_{3}$, las siguientes expresiones para los términos estabilizadores de 
las ecuaciones de la segunda y tercera componentes de la conservación de cantidad de movimiento pueden obtenerse en forma análoga:

$$
\begin{aligned}
& {\left[C_{i}\right]\left\{F_{i 2}^{C M}\right\}^{n}+\left(v_{2}\right)_{E}\left[\alpha_{i i}\right]\{p\}^{n}+(p)_{E}\left[\alpha_{2 i}\right]\left\{v_{i}\right\}^{n}} \\
& {\left[C_{i}\right]\left\{F_{i 3}^{C M}\right\}^{n}+\left(v_{3}\right)_{E}\left[\alpha_{i i}\right]\{p\}^{n}+(p)_{E}\left[\alpha_{3 i}\right]\left\{v_{i}\right\}^{n}}
\end{aligned}
$$

Ecuación de conservación de energía

Los términos estabilizadores de la ecuación de conservación de la energía se pueden obtener desarrollando el producto $\left[\boldsymbol{A}_{i}{ }^{n}\right]\left\{\boldsymbol{F}_{i}\right\}^{n}$ para la quinta ecuación, la que corresponde a $\rho e$, y considerando los valores de la quinta fila de cada una de las tres matrices $\boldsymbol{A}_{k}{ }^{n}$, de manera bastante similar a lo realizado con las ecuaciones de cantidad de movimiento. Deben tenerse en cuenta las expresiones (AI.3), (AI.17) y (AI.20). El desarrollo completo no se muestra aquí, dado que el procedimiento es bastante extenso y ya es conocido de las secciones anteriores, por lo que, a continuación, se muestran únicamente la expresión inicial y la expresión que finalmente se utiliza en las ecuaciones. Inicialmente se obtiene:

$$
\begin{gathered}
\left(\left(\bar{\gamma} V^{2}-\gamma e\right) v_{k}\right)_{E}\left[\alpha_{k i}\right]\left\{F_{i}^{M}\right\}^{n}+\left(\bar{\varepsilon} \delta_{k 1}-\bar{\gamma} v_{1} v_{k}\right)_{E}\left[\alpha_{k i}\right]\left\{F_{i 1}^{C M}\right\}^{n}+ \\
\left(\bar{\varepsilon} \delta_{k 2}-\bar{\gamma} v_{2} v_{k}\right)_{E}\left[\alpha_{k i}\right]\left\{F_{i 2}^{C M}\right\}^{n}+\left(\bar{\varepsilon} \delta_{k 3}-\bar{\gamma} v_{3} v_{k}\right)_{E}\left[\alpha_{k i}\right]\left\{F_{i 3}^{C M}\right\}^{n}+ \\
\left(\gamma v_{k}\right)_{E}\left[\alpha_{k i}\right]\left\{F_{i}^{E}\right\}^{n}
\end{gathered}
$$

Luego de realizado el trabajo de reducción puede expresarse:

$$
\left[C_{i}\right]\left\{F_{i}^{E}\right\}^{n}+(\bar{\varepsilon})_{E}\left[\alpha_{i i}\right]\{p\}^{n}+\left(\bar{\gamma} 0,5 V^{2}\right)_{E}\left[C_{i}\right]\left\{F_{i}^{M}\right\}^{n}
$$

Ecuación de transporte de $\tilde{v}(S A)$

Desarrollando el producto $\left[\boldsymbol{A}_{i}{ }^{n}\right]\left\{\boldsymbol{F}_{i}\right\}^{n}$ para la sexta y última ecuación y considerando los valores de la sexta fila de cada una de las matrices $\boldsymbol{A}_{k}{ }^{n} \mathrm{y}$, teniendo en cuenta la expresión (4.48), se tiene la siguiente expresión para los términos estabilizadores de la ecuación de transporte de SA:

$$
\begin{gathered}
-\left(v_{k} \tilde{v}\right)_{E}\left[\alpha_{k i}\right]\left\{F_{i}^{M}\right\}^{n}+\delta_{k 1}(\tilde{v})_{E}\left[\alpha_{k i}\right]\left\{F_{i 1}^{C M}\right\}^{n}+\delta_{k 2}(\tilde{v})_{E}\left[\alpha_{k i}\right]\left\{F_{i 2}^{C M}\right\}^{n}+ \\
\delta_{k 3}(\tilde{v})_{E}\left[\alpha_{k i}\right]\left\{F_{i 3}^{C M}\right\}^{n}+\left(v_{k}\right)_{E}\left[\alpha_{k i}\right]\left\{F_{i}^{S A}\right\}^{n}= \\
-(\tilde{v})_{E}\left[C_{i}\right]\left\{F_{i}^{M}\right\}^{n}+\left[C_{i}\right]\left\{F_{i}^{S A}\right\}^{n}+ \\
(\tilde{v})_{E}\left[\alpha_{1 i}\right]\left\{F_{i 1}^{C M}\right\}^{n}+(\tilde{v})_{E}\left[\alpha_{2 i}\right]\left\{F_{i 2}^{C M}\right\}^{n}+(\tilde{v})_{E}\left[\alpha_{3 i}\right]\left\{F_{i 3}^{C M}\right\}^{n}
\end{gathered}
$$

en donde las $\left[\alpha_{k i}\right]$ son matrices simétricas. Considerando que: 


$$
\left\{F_{i j}^{C M}\right\}^{n}=\left\{\rho v_{i} v_{j}+\delta_{i j} p\right\}^{n} ;\left\{F_{i}^{M}\right\}^{n}=\left\{\rho v_{i}\right\}^{n} ;\left\{F_{i}^{S A}\right\}^{n}=\left\{\rho \tilde{v} v_{i}\right\}^{n}
$$

puede sacarse $v_{j}$ del vector $\left\{F_{i j}^{C M}\right\}^{n}$ como un promedio de elemento, lo cual resulta en la siguiente expresión para los términos estabilizadores:

$$
\begin{gathered}
-(\tilde{v})_{E}\left[C_{i}\right]\left\{F_{i}^{M}\right\}^{n}+\left[C_{i}\right]\left\{F_{i}^{S A}\right\}^{n}+ \\
(\tilde{v})_{E}\left[\alpha_{1 i}\right]\left\{F_{i 1}^{C M}\right\}^{n}+(\tilde{v})_{E}\left[\alpha_{2 i}\right]\left\{F_{i 2}^{C M}\right\}^{n}+(\tilde{v})_{E}\left[\alpha_{3 i}\right]\left\{F_{i 3}^{C M}\right\}^{n}= \\
-(\tilde{v})_{E}\left[C_{i}\right]\left\{F_{i}^{M}\right\}^{n}+\left[C_{i}\right]\left\{F_{i}^{S A}\right\}^{n}+(\tilde{v})_{E}\left[C_{i}\right]\left\{F_{i}^{M}\right\}^{n}+(\tilde{v})_{E}\left[\alpha_{i i}\right]\{p\}^{n}
\end{gathered}
$$

Y, luego de la simplificación evidente en la última expresión, se tiene:

$$
\left[C_{i}\right]\left\{F_{i}^{S A}\right\}^{n}+(\tilde{v})_{E}\left[\alpha_{i i}\right]\{p\}^{n}
$$




\section{REFERENCIAS}

[1] Versteeg, H. K. and Malalasekera, W., An Introduction to Computational Fluid Dynamics: The Finite Volume Method (2nd Edition), Essex, Pearson Prentice Hall, 2007.

[2] Ferziger, J. H. and Dr. Perić, M., Computational Methods for Fluid Dynamics (3rd Edition), New York, Springer-Verlag Berlin Heidelberg, 2002.

[3] Zienkiewicz O. C., Taylor R. L., Nithiarasu P., The Finite Element Method for Fluid Dynamics (7th Edition), Oxford, Elsevier, 2013.

[4] Tennekes, H. and Lumley, J. L., A First Course in Turbulence. (15th Edition), London, MIT Press, 1972.

[5] Hinze, O., Turbulence, an Introduction to its Mechanism and Theory, New York, Mc Graw Hill, 1959.

[6] Wilcox, D. C., Turbulence Modeling for CFD (3rd Edition), California, D.C.W. Industries, 2006.

[7] Fröhlich, J. and von Terzi, D., Hybrid LES/RANS methods for the simulation of turbulent flows, Progress in Aerospace Sciences journal, (Volume 44, Issue 5), 2008, pp. 349-377.

[8] Cardiff, P. Demirdžić, I., Thirty years of the finite volume method for solid mechanics, at ResearchGate web page. 2018.

[9] Moukalled, F., Mangani L., Darwish M., The Finite Volume Method in Computational Fluid Dynamics. An Advanced Introduction with OpenFOAM® and Matlab, New York, Springer-Verlag Berlin Heidelberg, 2015.

[10] Bathe, K. J., Finite Element Procedures (2nd Edition), Watertown, Klaus-Jürgen Bathe, 2014.

[11] Antes, H., A Short Course on Boundary Element Methods, (Lecture by Dr. Ing. JensUwe Bohrnsen), Braunschweig, Institut fur Angewandte Mechanik, Technische Universitat, $\underline{2010 .}$

[12] Katz, A. J., Meshless Methods for Computational Fluid Dynamics. A dissertation for the degree of Doctor of Philosophy, Stanford University, 2009.

[13] Christie I., Griffiths D. F., Mitchell A. R., Zienkiewicz O. C., Finite element methods for second order differential equations with significant first derivatives. International Journal for Numerical Methods in Engineering (Vol. 10), Wiley Online Library, 1976, pp. 1389-1396.

[14] Hughes, T. Finite element method: linear static and dynamic finite element analysis, New Jersey, Prentice-Hall, 1987.

[15] Donea J. A Taylor-Galerkin method for convective transport problems. International Journal for Numerical Methods in Engineering, 20:101-119, 1984. 
[16] Burbridge, H.P. O esquema explícito de Taylor-Galerkin na simulação numérica de escoamentos compressiveis tridimensionais utilizando elementos finitos hexaédricos de oito nós. Tese de mestrado. UFRGS, Porto Alegre. 1999.

[17] Zienkiewicz, O.C. and Codina, R. A general algorithm for compressible and incompressible flow-Part I: The Split Charactestic-Based Scheme. International Journal for Numerical Methods in Fluids, 20:869-885, 1995.

[18] Massarotti, N., Arpino, F., Lewis, R.W., Nithiarasu, P. Explicit and semi-implicit CBS procedures for incompressible viscous flows. International journal for numerical methods in engineering, 66:1618-1640, 2006.

[19] Boonmarlert, P., Phongthanapanich, S., Dechaumpai, P. Combined characteristic-based split algorithm and Mesh Adaptation Technique for high-speed compressible flow analysis. Indian Journal of Engineering \& Material Sciences. 12:376-388, 2005.

[20] Zienkiewicz, O.C., Morgan K., Satya Sai B. V. K, Codina R., Vasquez M, A general algorithm for compressible and incompressible flow-Part II: Tests on the Explicit Form. International Journal for Numerical Methods in Fluids (Vol. 20), Wiley Online Library, 1995, pp. 887-913.

[21] Zhang, L., Zhou Y., Zhao L., Zhang D., Finite Element Method Using a CharacteristicBased Split for Numerical Simulation of a Carbonate Fracture-Cave Reservoir. Journal of Chemistry (Vol 2015), Hindawi Publishing Corporation, 2015.

[22] Kopp, P., Implementation of the Characteristic Based Split algorithm for the Shallow Water Equations in a high order finite element framework. Chair for Computation in Engineering, Bachelor's thesis. Technische Universitat Munchen, Munchen, 2014.

[23] Nithiarasu, P. and Zienwkiewicz, O.C., On stabilization of the CBS algorithm: Internal and external time steps. International Journal for Numerical Methods in Engineering (Vol. 48), 2000, Wiley Online Library, pp 875-880.

[24] Gresho P. M., Chan S. T., Lee R. L., Upson C. D., A modified finite element method for solving the time-dependent, incompressible Navier-Stokes equations (Part 1: Theory). International Journal for Numerical Methods in Fluids (Vol. 4), Wiley Online Library, 1984, pp. 557-598.

[25] Kawamoto, S. and Tanahashi, T., High-speed GSMAC-FEM for wind engineering. Computer Methods in Applied Mechanics and Engineering Journal (Vol. 112), Elsevier, 1994, pp. 219-226. 
[26] Molina R. C. and Huot J. P., A one-point integration finite element solver for the fast solution of the compressible Euler equation. Computer Methods in Applied Mechanics and Engineering Journal (Vol. 95), Elsevier, 1992, pp. 37-48.

[27] Santos, M. A. V., Simulação numérica de escoamentos compressíveis e sua interação com estruturas deformáveis, Tese de Doutorado, CPGEC, UFRGS, Porto Alegre, 1993.

[28] Azevedo, R. L., Análise de problemas de interação fluido estrutura usando o método dos elementos finitos com um acoplamento monolítico, Tese de Doutorado, CPGEC, UFRGS, Porto Alegre, 1999.

[29] Burbridge, H.P. and Awruch A.M. A Finite Element Taylor-Galerkin Scheme for 3-D Numerical Simulation of High Compressible Flow with Analytical Evaluation of Element Matrices. Hybrid Methods in Engineering. 2.I4:485-506, 2000.

[30] Liu C.B., The Characteristic Based Split (CBS) scheme for laminar and turbulent incompressible flow simulations. Doctoral Thesis, University of Wales, Swansea. 2005.

[31] Han, Z., Zhou D., Tu J., Fang C., He T., Flow over two side-by-side square cylinders by CBS finite element scheme of Spalart-Allmaras model. Ocean Engineering Journal (Vol. 87), Elsevier, 2014, pp. 40-49.

[32] Scungio, M., Arpino F., Stabile L., Buonanno G., Numerical Simulation of Ultrafine Particle Dispersion in Urban Street Canyons with the Spalart-Allmaras Turbulence Model. Aerosol and Air Quality Research (Vol. 13), Taiwan Association for Aerosol Research, 2013, pp.1423-1437.

[33] Turbulence Modeling Resource. Langley Research Center. Last Updated: 09/06/2019. https://turbmodels.larc.nasa.gov/spalart.html

[34] Bao, Y., Zhou D., Huang C., Wu Q., Chen X., Numerical prediction of aerodynamic characteristics of prismatic cylinder by finite element method with Spalart-Allmaras turbulence model. Computers and Structures (Vol. 89. Issues 3-4), Science Direct, Elsevier, 2011, 325-338.

[35] Nordanger, K., Holdahl R., Kvamsdal T., Kvarving A. M., Rasheed A., Simulation of airflow past a 2D NACA0015 airfoil using an isogeometric incompressible Navier-Stokes solver with the Spalart-Allmaras turbulence model. Computer Methods in Applied Mechanics and Engineering (Vol. 290), Science Direct, Elsevier, 2015, pp. 183-208.

[36] Pellerin, N., Leclaire S., Reggio M., An implementation of the Spalart-Allmaras turbulence model in a multi-domain lattice Boltzmann method for solving turbulent airfoil flows. Computers and Mathematics with Applications (Vol. 70), Science Direct, Elsevier, 2015, pp. 3001-3018. 
[37] Masuero, J. R., Computação Paralela na análise de problemas de engenharia utilizando o método dos elementos finitos. Tese de Doutorado. Programa de pós-graduação em Engenharia Civil. UFRGS, Porto Alegre, 2009.

[38] Dorneles, R. V. Particionamento de dominio e balanceamento de carga no modelo $H I D R A$. Tese de Doutorado. Programa de pós-graduação em Engenharia Civil. UFRGS, Porto Alegre, 2003.

[39] Demkowicz, L., Kurtz J., Pardo D., Paszenski M., Rachowicz W. Zdunek A., Computing with Hp-Adaptive Finite Elements (Vol. 2) Frontiers: Three Dimensional Elliptic and Maxwell Problems with Applications, Boca Raton, Chapman \& Hall/CRC, 2007.

[40] Allmaras S. R., Johnson F. T., Spalart P. R. Modifications and Clarifications for the Implementation of the Spalart-Allmaras Turbulence Model. Seventh International Conference on Computational Fluid Dynamics, ICCFD7-1902, 2012.

[41] White F., Viscous Fluid Flow, McGraw Hill Book Co. (First edition), N.Y., 1974.

[42] Asproulias I., RANS Modelling for Compressible Turbulent Flows Involving Shock Wave Boundary Layer Interactions, PHD Thesis, School of Mechanical Aerospace and Civil Engineering. The University of Manchester. 2014.

[43] Reynolds O., On the Dynamical Theory of Incompressible Viscous Fluids and the Determination of the Criterion. Philosophical Transactions of the Royal Society of London, Series A, Vol 186, pp 123-164, 1895.

[44] Favre. A., Equations Fondamentales des Fluides a Masse Variables en Ecoulements Turbulents. La Turbulence en Mécanique de Fluides. CNRS, pp 24-78. Paris 1962.

[45] Nichols R. H., Turbulence Models and Their Application to Complex Flows. University of Alabama at Birmingham (Rev 4.01). 2007.

[46] Boussinesq. J, Théorie de l'écoulement Tourbillonnant et Tumultueux des Liquides Dans les lits Rectilignes a Grande Section. Gauthier-Villars. 1897.

[47] Yoon, K. T., Moon, S. Y., Garcia S. A., Heard G. W., Chung T. J., Flowfield-dependent mixed explicit-implicit (FDMEI) methods for high and low speed and compressible and incompressible flows. Computer Methods in Applied Mechanics and Engineering., v. 151, p. 75-104, 1998.

[48] Huebner, K. H., Thornton, E. A., Byrom, T.G., The Finite Element Method for Engineers. Third Edition. John Wiley and Sons, Inc., pp 237-243, 1995.

[49] Mizukami, A., Some Integration Formulas for a Four-Node Isoparametric Element. Computer Methods in Applied Mechanics and Engineering. 59 (1986) 111-121.

[50] Argyris J., St. Doltsinis I, Friz H., Hermes space shuttle: exploration of reentry 
aerodynamics. Computer Methods in Applied Mechanics and Engineering, vol. 73, pp. 1-51. $\underline{1989 .}$

[51] Wilkinson, B., Allen, M., Parallel programming: Techniques and Applications Using Networked Workstations and Parallel Computers. ISBN 0136717101, 1st Ed., Prentice Hall, Saddle River, NJ 1999. 467p.

[52] Burbridge, H. P., Masuero J. R., Awruch, A. M., Implementación de códigos paralelizados en la simulación de flujos compresibles tridimensionales usando el método de los Elementos Finitos, CATE (Congreso Argentino de Tecnología Espacial), 2011.

[53] Mittal, S., Finite element computation of usteady viscous compressible flows. Computer Methods in Applied Mechanics and Engineering, vol. 157, pp. 151-175, 1998.

[54] Cambier, L., Computation of Viscous Transonic Flows Using an Unsteady Type Method and a Zonal Grid Refinement Technique. Numerical Simulation of Compressible NavierStokes Flow. Volume 18 of the series Notes on Numerical Fluid Mechanics pp 105-122, 1987. [55] Kussoy, M. I. and Horstman, C. C. Documentation of Two- and Three-Dimensional Hypersonic Shock Wave Boundary Layer Interaction Flows. NASA TM 101075. January 1989.

[56] Georgiadis, N.J., Rumsey Ch.L., Huang G.P. Revisiting Turbulence Model Validation for High-Mach Number Axisymmetric Compression Corner Flows. 53rd AIAA Aerospace Sciences Meeting. AIAA 2015-0316, 2015.

[57] Erb A. J. and Hosder S., Investigation of Turbulence Model Uncertainty for Supersonic/Hypersonic Shock Wave-Boundary Layer Interaction Predictions. 22nd AIAA International Space Planes and Hypersonics Systems and Technologies Conference. 2018.

[58] Burbridge, H. P., Scarabino A. E., Awruch A. M., Implementación del modelo de turbulencia de Spalart-Allmaras en un esquema explícito de Taylor-Galerkin para flujos compresibles, XII Congreso argentino de mecánica computacional, MECOM, 2018.

[59] Burbridge, H. P., Scarabino A. E., Awruch A. M., A Modified Taylor-Galerkin Scheme for Turbulent Compressible Flow Simulation., Revista Internacional de Métodos Numéricos para Cálculo y Diseño en Ingeniería, RIMNI. Volume 35, Issue 3, 2019. DOI: $\underline{10.23967 / \text { j.rimni.2019.09.006 }}$ 